\title{
Gold-catalyzed ascorbic acid-induced arylative carbocyclization of alkynes with aryldiazonium tetrafluoroborates.
}

Ignacio Medina-Mercado, ${ }^{[a]}$ Abraham Colin-Molina, ${ }^{[a]}$ José Enrique Barquera Lozada, ${ }^{[a]}$ Braulio Rodríguez-Molina ${ }^{[\mathrm{a}]}$ and Susana Porcel*[a]

${ }^{[a]}$ Instituto de Química, Universidad Nacional Autónoma de México, Circuito Exterior s/n, Ciudad Universitaria, Ciudad de México 04510, México.

\section{E-mail: sporcel@unam.mx}

\section{Table of contents}

General information 


\section{General Information.}

All reactions were carried out under a nitrogen atmosphere using standard Schlenk techniques. THF, and MeCN were dried by standard methods ${ }^{[1]}$ and freshly distilled prior to use. Dry DMSO was purchased from Aldrich and stored over molecular sieves (4 A, powder, activated, -325 mesh particle size). $\mathrm{CDCl}_{3}, \mathrm{DMSO}-d_{6}$ and commercial reagents were also purchased from Aldrich and used as received without further purification. IPrAuCl was purchased from Aldrich and stored under nitrogen atmosphere. Reactions were monitored by thin layer chromatography using TLC Alugram G/UV254 $0.20 \mathrm{~mm}$. Chromatography purifications were performed using flash grade silica gel (SDS Chromatogel 60 Acc, 40-60 $\mu \mathrm{m})$. NMR spectra were recorded at $25^{\circ} \mathrm{C}$ on a Jeol Eclipse $300 \mathrm{MHz}$, Bruker Fourier 300 MHz, Bruker Avance III $400 \mathrm{MHz}$ and Varian Unity Inova $500 \mathrm{MHz}$ spectrometers. Chemical shifts are reported in ppm and using the residual deuterated solvent as internal standard. High-resolution mass spectra (HRMS) were recorded on a Jeol The Accutof JMST100LC spectrometer using polyethylene glycol as internal standard. IR spectrum were recorded on ThermoFisher FT-IR Nicolet iS 50. Melting points were determined using a Reichert microscope apparatus and were uncorrected. 


\section{Synthesis of gold complexes}

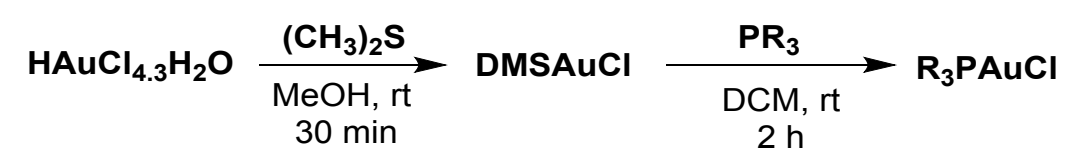

Step I. To a solution of $\mathrm{HAuCl}_{4} .3 \mathrm{H}_{2} \mathrm{O}(1.0638 \mathrm{~g}, 1.0$ equiv, $2.7 \mathrm{mmol})$ in $\mathrm{MeOH}(10 \mathrm{~mL})$, dimethylsulfide $(0.77 \mathrm{~mL}, 4.0$ equiv, $10.5 \mathrm{mmol})$ was added and the mixture was stirred for $30 \mathrm{~min}$ at room temperature. The solid was filtrated via cannula, washed with cooled $\mathrm{Et}_{2} \mathrm{O}$ $(3 \times 5 \mathrm{~mL})$ and dried under vacuo. The compound was obtained as a white solid in $94 \%$ yield (0.7501 g).

Step II. To a solution of DMSAuCl (1.0 equiv) in anhydrous DCM, the corresponding phosphine or phosphite (1.0 equiv) was added and the mixture was stirred for $2 \mathrm{~h}$ in the dark. Thereafter, the solvent was removed under reduced pressure and the resulting solid was dissolved with minimum amount of DCM and precipitated with hexane. The solid obtained was filtrated via cannula, dried in the high pump vacuum and stored under nitrogen.

$\mathbf{P h}_{3}$ PAuCl Prepared according to the general procedure (step II) using DMSAuCl (0.229 g, $0.78 \mathrm{mmol}), \mathrm{Ph}_{3} \mathrm{P}(0.2029 \mathrm{~g}, 0.78 \mathrm{mmol})$ and DCM $(10 \mathrm{~mL})$. The compound was obtained as a white solid in $97 \%$ yield (0.372 $\mathrm{g})$.

(2,4-tert-Bu $\left.\mathbf{P}_{2} \mathrm{PhO}\right)_{3} \mathrm{PAuCl}$ Prepared according to the general procedure (step II) using DMSAuCl (0.2519 g, $0.85 \mathrm{mmol})$, tris(2,4-di-tert-butylphenyl) phosphite (0.5532 g, 0.85 $\mathrm{mmol})$ and DCM $(10 \mathrm{~mL})$. The compound was obtained as a white solid in quantitative yield (0.7508 g).

\section{Synthesis of (but-2-yn-1-yloxy)benzene (1a). ${ }^{[2]}$}

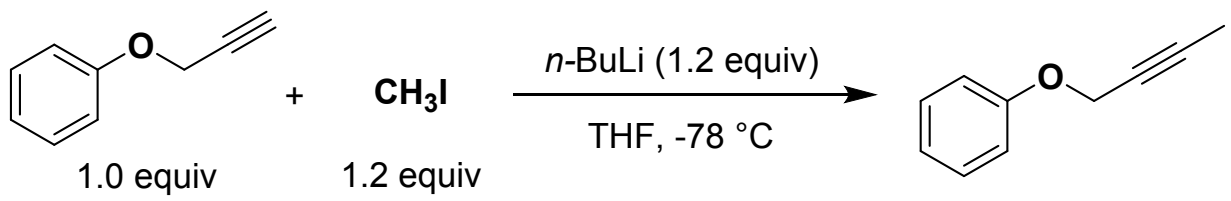

A solution of phenylpropargyl ether $(0.3962 \mathrm{~g}, 2.99 \mathrm{mmol})$ in anhydrous THF $(15 \mathrm{~mL})$ was cooled at $-78^{\circ} \mathrm{C}$. $n$-BuLi $(1.6 \mathrm{M}, 2.8 \mathrm{~mL}, 4.48 \mathrm{mmol})$ was added dropwise and the mixture was stirred for $2 \mathrm{~h} . \mathrm{CH}_{3} \mathrm{I}(0.56 \mathrm{~mL}, 8.99 \mathrm{mmol})$ was added and the mixture was allowed to 
stir at room temperature for $1 \mathrm{~h}$. The solvent was removed under vacuum, and the residue obtained was dissolved with $\mathrm{CH}_{2} \mathrm{Cl}_{2}(20 \mathrm{~mL})$ and washed with brine. The organic layer was dried over $\mathrm{Na}_{2} \mathrm{SO}_{4}$ and concentrated. The crude mixture was purified by column chromatography on silica gel using hexane/EtOAc 98:2 as eluent. The desired product was obtained as colorless oil in 76\% yield (0.3351 g). ${ }^{1} \mathbf{H}$ NMR (400 $\left.\mathbf{M H z}, \mathbf{C D C l}_{3}\right) \delta 7.34-7.27$ $(\mathrm{m}, 2 \mathrm{H}), 7.04-6.92(\mathrm{~m}, 3 \mathrm{H}), 4.65$ (s, 2H), 1.88 (s, 3H). ${ }^{13} \mathbf{C}$ NMR (100 MHz, CDCl$) \delta$ $157.95,129.54,121.33,114.94,83.79,74.20,56.46,3.85$.

\section{Synthesis of (hept-2-yn-1-yloxy)benzene (3a). ${ }^{[2]}$}

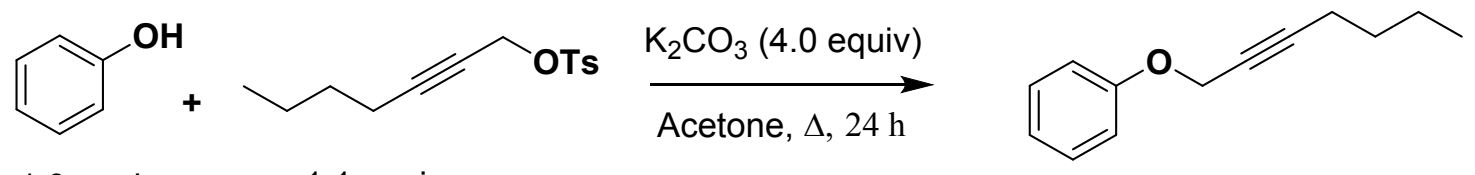

1.0 equiv $\quad 1.1$ equiv

To a solution of phenol (01376 g, 1.0 equiv, $1.46 \mathrm{mmol})$ and hept-2-yn-1-yl-4methylbenzenesulfonate $(0.4286 \mathrm{~g}, 1.1$ equiv, $1.6 \mathrm{mmol})$ in acetone $(20 \mathrm{~mL}), \mathrm{K}_{2} \mathrm{CO}_{3}(0.8087$ g, 4.0 equiv, $5.8 \mathrm{mmol}$ ) was added and the mixture was heated under reflux for $24 \mathrm{~h}$. Then, it was washed with water $(20 \mathrm{~mL})$ and extracted with EtOAc $(3 \times 20 \mathrm{~mL})$. The organic layer was dried over $\mathrm{Na}_{2} \mathrm{SO}_{4}$ and concentrated under vacuum. The crude mixture was purified by column chromatography on silica gel using hexane/EtOAc 9:1 as eluent. The corresponding alkyne was obtained as colorless oil in 65\% yield (0.1776 g). ${ }^{1} \mathbf{H}$ NMR (300 MHz, CDCl $\left.\mathbf{~}_{3}\right)$ $\delta 7.35-7.26(\mathrm{~m}, 2 \mathrm{H}), 7.05-6.93(\mathrm{~m}, 3 \mathrm{H}), 4.69(\mathrm{t}, J=2.2 \mathrm{~Hz}, 2 \mathrm{H}), 2.25(\mathrm{tt}, J=7.0,2.2 \mathrm{~Hz}$, 2H), $1.57-1.46(\mathrm{~m}, 2 \mathrm{H}), 1.45-1.34(\mathrm{~m}, 2 \mathrm{H}), 0.91(\mathrm{t}, J=7.2 \mathrm{~Hz}, 3 \mathrm{H}) .{ }^{13} \mathbf{C}$ NMR (75 MHz, $\left.\mathbf{C D C l}_{3}\right) \delta 157.98,129.46,121.28,115.04,88.34,75.02,56.54,30.63,22.02,18.60,13.67$.

\section{Synthesis of alkynes 2a, 4-10a, 13-17a, 19-20a.}

\section{General procedure.}

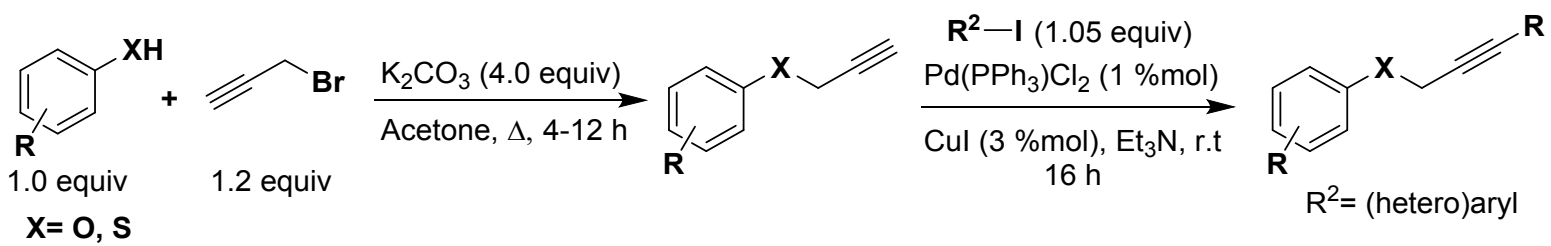

Step I. To a solution of the corresponding phenol or thiophenol (1.0 equiv) in acetone, was added $\mathrm{K}_{2} \mathrm{CO}_{3}$ (4.0 equiv) and the mixture was heated under reflux for $30 \mathrm{~min}$. Then, 
propargyl bromide (1.2 equiv) was added and the heating was continued for $12 \mathrm{~h}$. The mixture was washed with water and extracted with EtOAc. The organic layer was dried over $\mathrm{Na}_{2} \mathrm{SO}_{4}$ and concentrated under vacuum to obtain the desired arylpropargyl ether (73-96 \% yields) which was used without further purification in the next step.

Step II. To a solution of arylpropargyl ether (1.0 equiv), $\mathrm{Pd}\left(\mathrm{PPh}_{3}\right) \mathrm{Cl}_{2}(0.01$ equiv) and $\mathrm{Ar}-\mathrm{I}$ (1.05 equiv) in $\mathrm{Et}_{3} \mathrm{~N}(5 \mathrm{~mL})$ was added $\mathrm{CuI}$ (0.03 equiv); the mixture was stirred at room temperature for $16 \mathrm{~h}$. Thereafter was washed with water and extracted with EtOAc. The organic layer was dried over $\mathrm{Na}_{2} \mathrm{SO}_{4}$ and concentrated under vacuum. The crude obtained was purified by column chromatography on silica gel using hexanes/EtOAc as eluent.

\section{1-Methoxy-4-(3-phenoxyprop-1-yn-1-yl)benzene (2a). ${ }^{[3]}$}

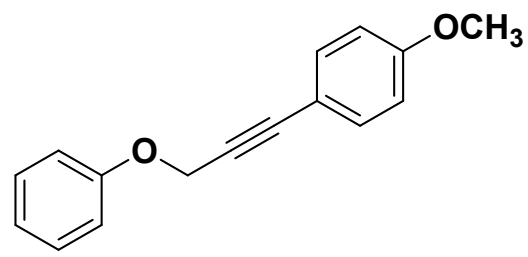

Prepared according to the general procedure (step II) using (prop-2-yn-1-yloxy)benzene (0.4791 g) 4-iodoanisole (0.9332 g) $\mathrm{Pd}\left(\mathrm{PPh}_{3}\right)_{2} \mathrm{Cl}_{2}(25.4 \mathrm{mg})$ and $\mathrm{CuI}$ (20.7 mg). Purification by column chromatography (silica gel, hexanes/EtOAc 95:5) gave the product $(0.6697 \mathrm{~g}$, 77\%) as a white solid. ${ }^{1} \mathbf{H}$ NMR (300 $\left.\mathbf{M H z}, \mathbf{C D C l}_{3}\right) \delta 7.42-7.37$ (m, 2H), 7.37 - 7.29 (m, 2H), $7.08-7.02(\mathrm{~m}, 2 \mathrm{H}), 7.02-6.97(\mathrm{~m}, 1 \mathrm{H}), 6.89-6.80(\mathrm{~m}, 2 \mathrm{H}), 4.91(\mathrm{~s}, 2 \mathrm{H}), 3.81(\mathrm{~s}$, 3H). ${ }^{13}$ C NMR (75 MHz, $\left.\mathbf{C D C l}_{3}\right) \delta 160.01,157.99,133.48,129.58,121.47,115.09,114.49$, $114.03,87.23,82.70,56.83,55.39$.

\section{(3-Phenoxyprop-1-yn-1-yl)benzene (4a). ${ }^{[3]}$}

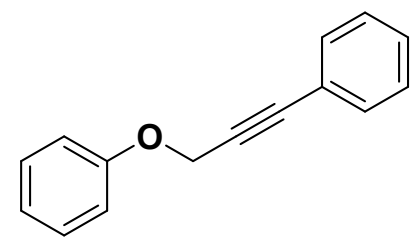

Prepared according to the general procedure (step II) using prop-2-yn-1-yloxy)benzene (0.2 g) iodobenzene $(0.3087 \mathrm{~g}) \mathrm{Pd}\left(\mathrm{PPh}_{3}\right)_{2} \mathrm{Cl}_{2}(10.6 \mathrm{mg})$ and $\mathrm{CuI}(6.3 \mathrm{mg})$. Purification by column 
chromatography (silica gel, hexanes/EtOAc 95:5) gave the product $(0.2356 \mathrm{~g}, 73 \%)$ as a white solid. ${ }^{1} \mathbf{H}$ NMR (300 MHz, $\left.\mathbf{C D C l}_{3}\right) \delta 7.51-7.40(\mathrm{~m}, 2 \mathrm{H}), 7.38$ - $7.24(\mathrm{~m}, 5 \mathrm{H}), 7.10$ 6.96 (m, 3H), 4.94 (s, 2H). ${ }^{13} \mathbf{C}$ NMR (75 MHz, $\left.\mathbf{C D C l}_{3}\right) \delta 157.94,131.94,129.60,128.78$, $128.40,122.44,121.55,115.12,87.26,84.10,56.75$.

\section{1-(3-Phenoxyprop-1-yn-1-yl)naphthalene (5a).}

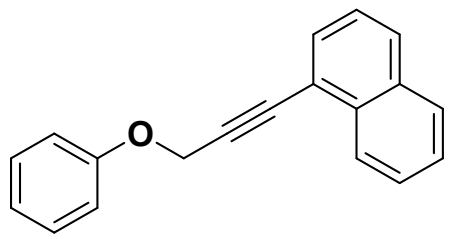

Prepared according to the general procedure (step II) using prop-2-yn-1-yloxy)benzene (0.2 g) 1-iodonaphtalene $(0.3964 \mathrm{~g}) \mathrm{Pd}\left(\mathrm{PPh}_{3}\right)_{2} \mathrm{Cl}_{2}(10.6 \mathrm{mg})$ and $\mathrm{CuI}(6.3 \mathrm{mg})$. Purification by column chromatography (silica gel, hexanes/EtOAc 95:5) gave the product $(0.3081 \mathrm{~g}, 73 \%)$ as a pale yellow oil. ${ }^{1} \mathbf{H}$ NMR (400 $\left.\mathbf{M H z}, \mathbf{C D C l}_{3}\right) \delta 8.30-8.22(\mathrm{~m}, 1 \mathrm{H}), 7.88-7.82$ (m, 2H), $7.71(\mathrm{dd}, J=7.1,1.0 \mathrm{~Hz}, 1 \mathrm{H}), 7.58-7.50(\mathrm{~m}, 2 \mathrm{H}), 7.46-7.35(\mathrm{~m}, 3 \mathrm{H}), 7.18-7.13(\mathrm{~m}$, 2H), 7.10 - $7.04(\mathrm{~m}, 1 \mathrm{H}), 5.10$ (s, 2H). ${ }^{13} \mathbf{C}$ NMR (100 MHz, $\left.\mathbf{C D C l}_{3}\right) \delta$ 157.90, 133.43, 133.19 , 130.86, 129.63, 129.25, 128.35, 126.97, 126.55, 126.19, 125.22, 121.64, 120.06, 115.34, 88.97, 85.48, 56.91. HRMS-DART calculated for $\mathrm{C}_{19} \mathrm{H}_{15} \mathrm{O}[\mathrm{M}+\mathrm{H}]^{+}:$259.11229; found: 259.11144. IR (ATR): 3056, 2908, 2858, 2228, 1597, 1586, 1493, 1368, 1211, 1172, $1030,1012,992,798,772,750,689,565 \mathrm{~cm}^{-1}$.

\section{1-Methyl-2-(3-phenoxyprop-1-yn-1-yl)benzene (6a).}

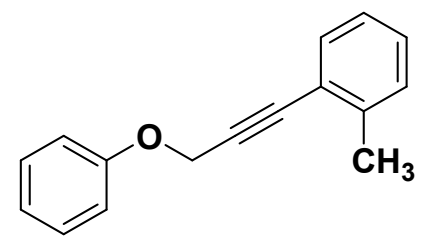

Prepared according to the general procedure (step II) using prop-2-yn-1-yloxy)benzene (0.3

g) 2-iodotoluene $(0.505 \mathrm{~g}) \mathrm{Pd}\left(\mathrm{PPh}_{3}\right)_{2} \mathrm{Cl}_{2}(15.9 \mathrm{mg})$ and $\mathrm{CuI}(9.5 \mathrm{mg})$. Purification by column chromatography (silica gel, hexanes/EtOAc 95:5) gave the product $(0.5045 \mathrm{~g}, 62 \%$ ) as yellow oil. ${ }^{1}$ H NMR (300 MHz, $\left.\mathbf{C D C l}_{3}\right) \delta 7.45$ (d, $J=7.5$ Hz, 1H), 7.40 - 7.31 (m, 2H), 7.30 - 6.99 (m, 6H), 5.01 (s, 2H), 2.42 (s, 3H). ${ }^{13} \mathbf{C}$ NMR (75 MHz, $\mathbf{C D C l}_{3}$ ) $\delta$ 157.78, 140.55, 132.17, $130.33,129.45,128.67,125.52,122.13,121.43,115.15,87.86,86.17,56.67,20.65$. HRMS- 
DART calculated for $\mathrm{C}_{16} \mathrm{H}_{15} \mathrm{O}[\mathrm{M}+\mathrm{H}]^{+}:$223.11229; found: 223.11129. IR (ATR): 3062, 3027, 2919, 2859, 15978, 1588, 1485, 1455, 1372, 1335, 1212, 1173, 1032, 1016, 749, 689, $507 \mathrm{~cm}^{-1}$.

\section{1-Methoxy-3-(3-phenoxyprop-1-yn-1-yl)benzene (7a). ${ }^{[4]}$}

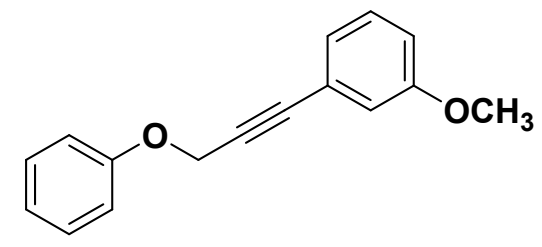

Prepared according to the general procedure (step II) using prop-2-yn-1-yloxy)benzene ( 0.2 g) 3-iodoanisole (0.3606 g) $\mathrm{Pd}\left(\mathrm{PPh}_{3}\right)_{2} \mathrm{Cl}_{2}(10.6 \mathrm{mg})$ and $\mathrm{CuI}(6.3 \mathrm{mg})$. Purification by column chromatography (silica gel, hexanes/EtOAc 95:5) gave the product $(0.298 \mathrm{~g}, 82 \%)$ as pale yellow oil. ${ }^{1} \mathbf{H}$ NMR (400 MHz, $\left.\mathbf{C D C l}_{3}\right) 7.27-7.21(\mathrm{~m}, 2 \mathrm{H}), 7.14(\mathrm{dd}, J=15.9,8.0 \mathrm{~Hz}$, 1H), $6.98-6.94(\mathrm{~m}, 3 \mathrm{H}), 6.94-6.91(\mathrm{~m}, 1 \mathrm{H}), 6.89(\mathrm{ddd}, J=3.9,2.2,1.2 \mathrm{~Hz}, 1 \mathrm{H}), 6.80$ (ddd, $J=8.3,2.6,0.9 \mathrm{~Hz}, 1 \mathrm{H}), 4.83$ (s, 2H), 3.70 (s, 3H). ${ }^{13} \mathbf{C} \mathbf{~ N M R}\left(\mathbf{1 0 0} \mathbf{~ M H z}, \mathbf{C D C l}_{3}\right) \delta 159.38$, 157.93, 129.61, 129.49, 124.45, 123.40, 121.57, 116.76, 115.39, 115.10, 87.17, 83.91, 56.74, 55.40 .

\section{2-(3-Phenoxyprop-1-yn-1-yl)thiophene (9a). ${ }^{[5]}$}

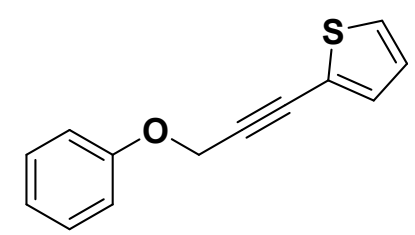

Prepared according to the general procedure (step II) using prop-2-yn-1-yloxy)benzene (0.2 g) 2-iodothiophene (0.3243 g) $\mathrm{Pd}\left(\mathrm{PPh}_{3}\right)_{2} \mathrm{Cl}_{2}(10.6 \mathrm{mg})$ and $\mathrm{CuI}(6.3 \mathrm{mg})$. Purification by column chromatography (silica gel, hexanes/EtOAc 95:5) gave the product $(0.2077 \mathrm{~g}, 76 \%)$ as beige solid. ${ }^{1} \mathbf{H}$ NMR (300 MHz, $\left.\mathbf{C D C l}_{3}\right) \delta 7.38-7.29(\mathrm{~m}, 2 \mathrm{H}), 7.29-7.23(\mathrm{~m}, 2 \mathrm{H}), 7.07$ - $7.00(\mathrm{~m}, 3 \mathrm{H}), 7.00-6.94(\mathrm{~m}, 1 \mathrm{H}), 4.93$ (s, 2H). ${ }^{13} \mathbf{C}$ NMR (75 MHz, $\left.\mathbf{C D C l}_{3}\right) \delta 157.75$, $132.80,129.52,127.68,126.96,122.16,121.51,114.96,87.97,80.47,56.68$. 


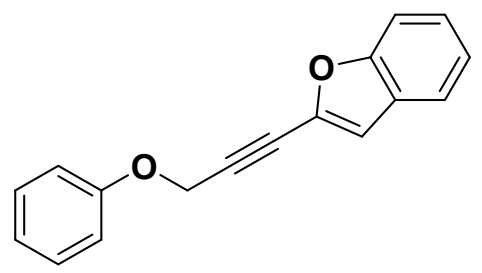

Prepared according to the general procedure (step II) using prop-2-yn-1-yloxy)benzene (0.3 g, $2.26 \mathrm{mmol}$ ) 2-iodobenzofuran (0.5932 g, $2.43 \mathrm{mmol}) \mathrm{Pd}\left(\mathrm{PPh}_{3}\right)_{2} \mathrm{Cl}_{2}$ (16 mg, $\left.0.02 \mathrm{mmol}\right)$ and $\mathrm{CuI}$ (13 mg, 0.06). Purification by column chromatography (silica gel, hexanes/EtOAc 98:2) gave the product $(0.4063 \mathrm{~g}, 72 \%)$ as beige solid. m.p. $58-60{ }^{\circ} \mathrm{C}{ }^{\mathbf{1}} \mathbf{H}$ NMR (300 MHz, $\left.\mathbf{C D C l}_{3}\right) \delta 7.56(\mathrm{ddd}, J=7.7,1.3,0.7 \mathrm{~Hz}, 1 \mathrm{H}), 7.46(\mathrm{dq}, J=8.3,0.9 \mathrm{~Hz}, 1 \mathrm{H}), 7.39-7.31(\mathrm{~m}$, $3 \mathrm{H}), 7.29-7.21(\mathrm{~m}, 1 \mathrm{H}), 7.09-7.03(\mathrm{~m}, 3 \mathrm{H}), 7.00(\mathrm{dd}, J=9.1,0.9 \mathrm{~Hz}, 1 \mathrm{H}), 4.99(\mathrm{~s}, 2 \mathrm{H})$. ${ }^{13}$ C NMR (75 MHz, $\left.\mathbf{C D C l}_{3}\right) \delta 157.72,154.98,137.85,129.71,127.46,126.00,123.48$, 121.84, 121.47, 115.06, 112.69, 111.43, 90.36, 77.72, 56.47. HRMS-DART calculated for $\mathrm{C}_{17} \mathrm{H}_{13} \mathrm{O}_{2}[\mathrm{M}+\mathrm{H}]^{+}:$249.09155; found: 249.09075. IR (ATR): 3062, 3039, 2913, 2856, 1597, 1588, 1566, 1493, 1448, 1369, 1304, 1256, 1210, 1173, 1159, 1108, 1079, 1033, 1009, 981, $883,812,747,689,644,613,546,506 \mathrm{~cm}^{-1}$.

\section{1-Methoxy-4-(3-(4-methoxyphenoxy)prop-1-yn-1-yl)benzene (13a). ${ }^{[6]}$}

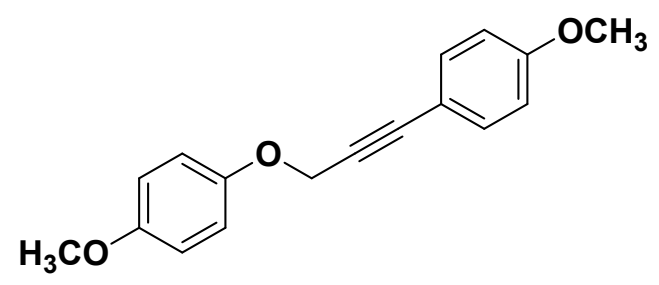

Prepared according to the general procedure (step II) using 1-methoxy-4-(prop-2-yn-1yloxy)benzene (0.5838 g) 4-iodoanisole (0.8835 g) $\mathrm{Pd}\left(\mathrm{PPh}_{3}\right)_{2} \mathrm{Cl}_{2}(25.2 \mathrm{mg})$ and $\mathrm{CuI}(20.5$ mg). Purification by column chromatography (silica gel, hexanes/EtOAc 9:1) gave the product (0.7418 g, 77\%) as a white solid. ${ }^{1} \mathbf{H}$ NMR (300 $\left.\mathbf{~ M H z , ~} \mathbf{C D C l}_{3}\right) \delta 7.42-7.35$ (m, 2H), $7.03-6.95(\mathrm{~m}, 2 \mathrm{H}), 6.91-6.79(\mathrm{~m}, 5 \mathrm{H}), 4.85(\mathrm{~s}, 2 \mathrm{H}), 3.80(\mathrm{~s}, 3 \mathrm{H}), 3.78(\mathrm{~s}, 3 \mathrm{H}) .{ }^{13} \mathrm{C}$ NMR (75 MHz, $\left.\mathbf{C D C l}_{3}\right) \delta 159.98,154.49,152.16,133.43,116.35,114.72,114.57,114.03$, $87.10,83.00,57.73,55.79,55.37$. 


\section{1-(Tert-butyl)-4-((3-(4-methoxyphenyl)prop-2-yn-1-yl)oxy)benzene (14a).}

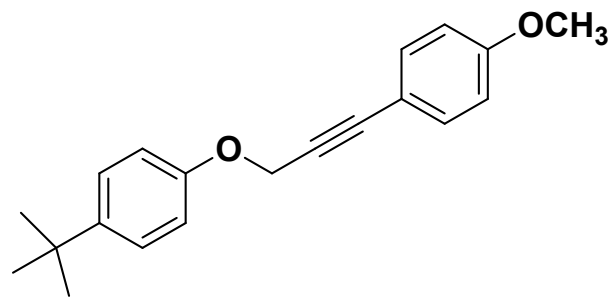

Prepared according to the general procedure (step II) using 1-(tert-butyl)-4-(prop-2-yn-1yloxy)benzene (0.3262 g) 4-iodoanisole (0.4258 g) $\mathrm{Pd}\left(\mathrm{PPh}_{3}\right)_{2} \mathrm{Cl}_{2}(12.1 \mathrm{mg})$ and $\mathrm{CuI}(9.9 \mathrm{mg})$. Purification by column chromatography (silica gel, hexanes/EtOAc 9:1) gave the product (0.4617 g, 90\%) as a white solid. m.p. $113-115{ }^{\circ} \mathrm{C} .{ }^{1} \mathbf{H}$ NMR (300 MHz, $\left.\mathbf{C D C l}_{3}\right) \delta 7.44-$ $7.38(\mathrm{~m}, 2 \mathrm{H}), 7.37-7.31(\mathrm{~m}, 2 \mathrm{H}), 7.02-6.95(\mathrm{~m}, 2 \mathrm{H}), 6.87-6.81(\mathrm{~m}, 2 \mathrm{H}), 4.89(\mathrm{~s}, 2 \mathrm{H})$, 3.81 (s, 3H), 1.32 (s, 9H). ${ }^{13}$ C NMR (75 MHz, $\left.\mathbf{C D C l}_{3}\right) \delta$ 159.98, 155.82, 144.16, 133.49, 126.38, 114.59, 114.54, 114.02, 87.07, 82.96, 56.96, 55.39, 34.24, 31.65. HRMS-DART calculated for $\mathrm{C}_{20} \mathrm{H}_{23} \mathrm{O}_{2}[\mathrm{M}+\mathrm{H}]^{+}:$295.16980; found: 295.16979. IR (ATR): 3011, 2959, 2904, 2864, 2226, 1604, 1568, 1506, 1462, 1442, 1376, 1292, 1248, 1233, 1186, 1172, 1105 , $1026,1011,984,924,832,817,638,554,536,519 \mathrm{~cm}^{-1}$.

\section{1-(Benzyloxy)-4-((3-(4-methoxyphenyl)prop-2-yn-1-yl)oxy)benzene (15a).}

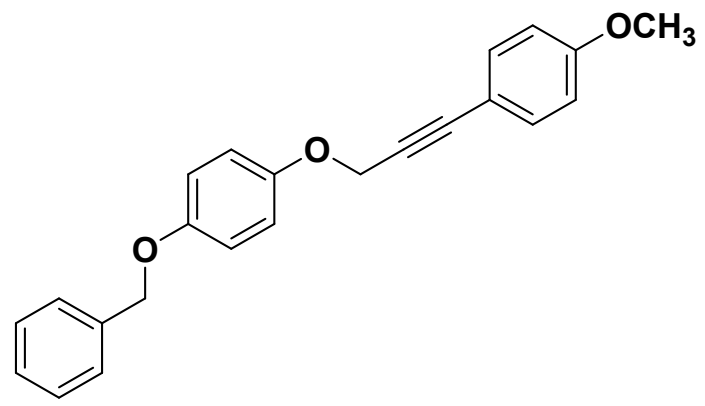

Prepared according to the general procedure (step II) using 1-(benzyloxy)-4-(prop-2-yn-1yloxy)benzene (0.3371 g) 4-iodoanisole (0.3476 g) $\mathrm{Pd}\left(\mathrm{PPh}_{3}\right)_{2} \mathrm{Cl}_{2}(9.9 \mathrm{mg})$ and $\mathrm{CuI}(8.1 \mathrm{mg})$. Purification by column chromatography (silica gel, hexanes/EtOAc 95:5) gave the product (0.2082 g, 43\%) as a white solid. m.p. 106-108 ${ }^{\circ} \mathrm{C} .{ }^{1} \mathbf{H}$ NMR (300 MHz, CDCl $) \delta 7.48-$ $7.30(\mathrm{~m}, 7 \mathrm{H}), 7.02-6.92(\mathrm{~m}, 4 \mathrm{H}), 6.87-6.81(\mathrm{~m}, 2 \mathrm{H}), 5.04(\mathrm{~s}, 2 \mathrm{H}), 4.86(\mathrm{~s}, 2 \mathrm{H}), 3.81(\mathrm{~s}$, 3H). ${ }^{13}$ C NMR (75 MHz, $\left.\mathbf{C D C l}_{3}\right) \delta 159.97,153.66,152.33,137.34,133.45,128.68,128.03$, 
127.61, 116.28, 115.85, 114.53, 114.02, 87.13, 82.95, 70.72, 57.65, 55.38. HRMS-DART calculated for $\mathrm{C}_{23} \mathrm{H}_{21} \mathrm{O}_{3}[\mathrm{M}+\mathrm{H}]^{+}:$345.14907; found: 345.14817. IR (ATR): 3003, 2964, 2908, 2866, 2836, 2229, 1602, 1568, 1504, 1454, 1439, 1376, 1288, 1249, 1221, 1181, 1173, $1109,1019,987,923,825,747,700,672,528,483 \mathrm{~cm}^{-1}$.

\section{4-((3-(4-Methoxyphenyl)prop-2-yn-1-yl)oxy)-1,1'-biphenyl (16a). ${ }^{[7]}$}

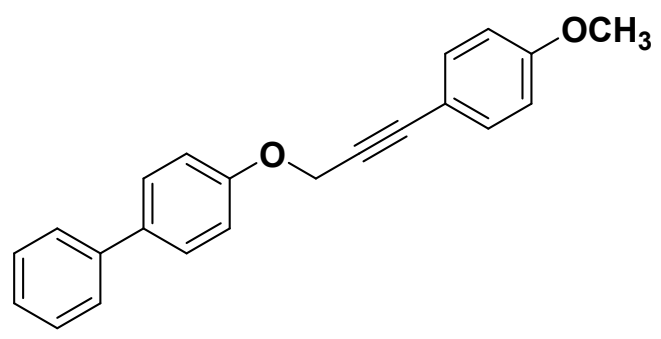

Prepared according to the general procedure (step II) using 4-(prop-2-yn-1-yloxy)-1,1'biphenyl (0.5862 g) 4-iodoanisole (0.6916 g) $\mathrm{Pd}\left(\mathrm{PPh}_{3}\right)_{2} \mathrm{Cl}_{2}(19.7 \mathrm{mg})$ and $\mathrm{CuI}(16 \mathrm{mg})$. Purification by column chromatography (silica gel, hexanes/EtOAc 9:1) gave the product $(0.7121 \mathrm{~g}, 80 \%)$ as a white solid. ${ }^{1} \mathbf{H}$ NMR (400 $\left.\mathbf{~ M H z}, \mathbf{C D C l}_{3}\right) \delta 7.62-7.53(\mathrm{~m}, 4 \mathrm{H}), 7.47$ $-7.38(\mathrm{~m}, 4 \mathrm{H}), 7.36-7.29(\mathrm{~m}, 1 \mathrm{H}), 7.18-7.08(\mathrm{~m}, 2 \mathrm{H}), 6.87-6.82(\mathrm{~m}, 2 \mathrm{H}), 4.95(\mathrm{~s}, 2 \mathrm{H})$, 3.81 (s, 3H). ${ }^{13} \mathbf{C}$ NMR (100 MHz, $\left.\mathbf{C D C l}_{3}\right) \delta 160.03,157.54,140.85,134.55,133.50,128.85$, $128.27,126.90,126.88,115.39,114.45,114.05,87.40,82.63,57.01,55.40$.

\section{1-Bromo-4-((3-(4-methoxyphenyl)prop-2-yn-1-yl)oxy)benzene (17a). ${ }^{[8]}$}

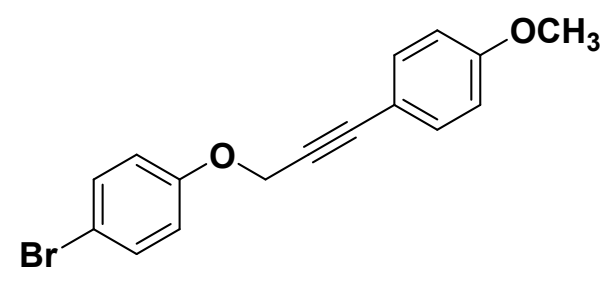

Prepared according to the general procedure (step II) using 1-bromo-4-(prop-2-yn-1yloxy)benzene (0.5597 g) 4-iodoanisole (0.6516 g) $\mathrm{Pd}\left(\mathrm{PPh}_{3}\right)_{2} \mathrm{Cl}_{2}(18.6 \mathrm{mg})$ and $\mathrm{CuI}(15.1$ $\mathrm{mg}$ ). Purification by column chromatography (silica gel, hexanes/EtOAc 95:5) gave the product $(0.6635 \mathrm{~g}, 79 \%)$ as a white solid. ${ }^{1} \mathbf{H}$ NMR (300 $\left.\mathbf{~ M H z}, \mathbf{C D C l}_{3}\right) \delta 7.44-7.33(\mathrm{~m}$, 4H), $6.95-6.88$ (m, 2H), $6.86-6.80$ (m, 2H), 4.87 (s, 2H), 3.80 (s, 3H). ${ }^{13} \mathbf{C}$ NMR (75 MHz, CDCl $\left._{3}\right) \delta 160.11,157.04,133.48,132.40,116.97,114.24,114.08,113.74,87.68,82.14$, $57.08,55.41$. 


\section{1-(4-((3-(4-Methoxyphenyl)prop-2-yn-1-yl)oxy)phenyl)ethan-1-one (19a). ${ }^{[7]}$}

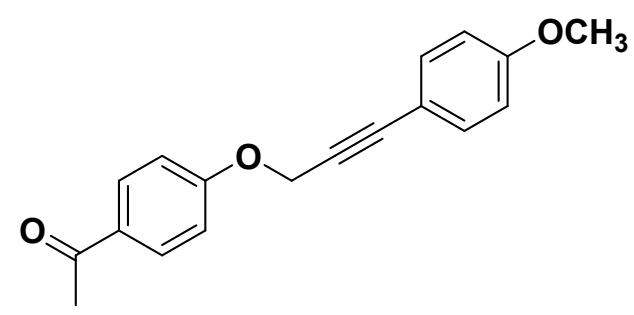

Prepared according to the general procedure (step II) using 1-(4-(prop-2-yn-1yloxy)phenyl)ethan-1-one (0.6318 g) 4-iodoanisole (0.8248 g) $\mathrm{Pd}\left(\mathrm{PPh}_{3}\right)_{2} \mathrm{Cl}_{2}(23.6 \mathrm{mg})$ and $\mathrm{CuI}$ (19.2 mg). Purification by column chromatography (silica gel, hexanes/EtOAc 8:2) gave the product (0.6625 g, 70\%) as a pale yellow solid. ${ }^{1} \mathbf{H}$ NMR (400 $\left.\mathbf{M H z}, \mathbf{C D C l}_{3}\right) \delta 8.01-$ $7.89(\mathrm{~m}, 2 \mathrm{H}), 7.41-7.30(\mathrm{~m}, 2 \mathrm{H}), 7.13-7.01(\mathrm{~m}, 2 \mathrm{H}), 6.87-6.78(\mathrm{~m}, 2 \mathrm{H}), 4.96(\mathrm{~s}, 2 \mathrm{H})$, 3.79 (s, 3H), 2.56 (s, 3H). ${ }^{13} \mathbf{C}$ NMR (100 MHz, $\left.\mathbf{C D C l}_{3}\right) \delta 196.88,161.74,160.14,133.48$, $130.93,130.62,114.75,114.07,87.93,81.78,56.95,55.40,26.49$.

\section{(3-(4-methoxyphenyl)prop-2-yn-1-yl)(phenyl)sulfane (20a).}

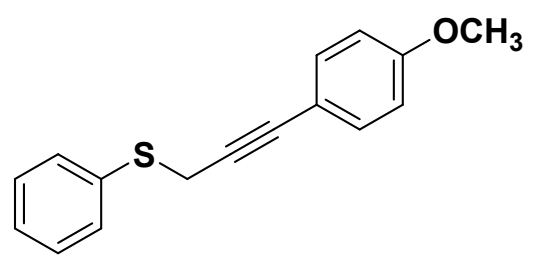

Prepared according to the general procedure (step II) using (3-(4-methoxyphenyl)prop-2-yn1-yl)(phenyl)sulfane (0.4182 g) 4-iodoanisole (0.6603 g) $\mathrm{Pd}\left(\mathrm{PPh}_{3}\right)_{2} \mathrm{Cl}_{2}(20 \mathrm{mg})$ and $\mathrm{CuI}(16$ mg). Purification by column chromatography (silica gel, hexanes/EtOAc 98:2) gave the product (0.4319 g, 60\%) as a pale yellow solid. m.p. 35-37 ${ }^{\circ}$ C. ${ }^{1} \mathbf{H}$ NMR (400 $\left.\mathbf{~ M H z , ~} \mathbf{C D C l}_{3}\right)$ $\delta 7.56-7.51(\mathrm{~m}, 2 \mathrm{H}), 7.39-7.34(\mathrm{~m}, 2 \mathrm{H}), 7.34-7.31(\mathrm{~m}, 2 \mathrm{H}), 7.31-7.26(\mathrm{~m}, 1 \mathrm{H}), 6.87-$ $6.81(\mathrm{~m}, 2 \mathrm{H}), 3.87$ (s, 2H), 3.82 (s, 3H). ${ }^{13} \mathbf{C}$ NMR (100 MHz, $\left.\mathbf{C D C l}_{3}\right) \delta$ 159.64, 135.45, 133.20, 130.56, 129.02, 127.00, 115.17, 113.97, 83.80, 83.70, 55.38, 24.01. HRMS-DART calculated for $\mathrm{C}_{16} \mathrm{H}_{15} \mathrm{OS}[\mathrm{M}+\mathrm{H}]^{+}:$255.08436; found: 255.08335. IR (ATR): 3059, 3006, 2960, 2930, 2835, 1602, 1509, 1478, 1435, 1292, 1250, 1218, 1172, 1107, 1025, 831, 812, $751,741,687,537,476 \mathrm{~cm}^{-1}$. 


\section{Synthesis of (3-phenoxyprop-1-yn-1-yl)(phenyl)sulfide (11a).}

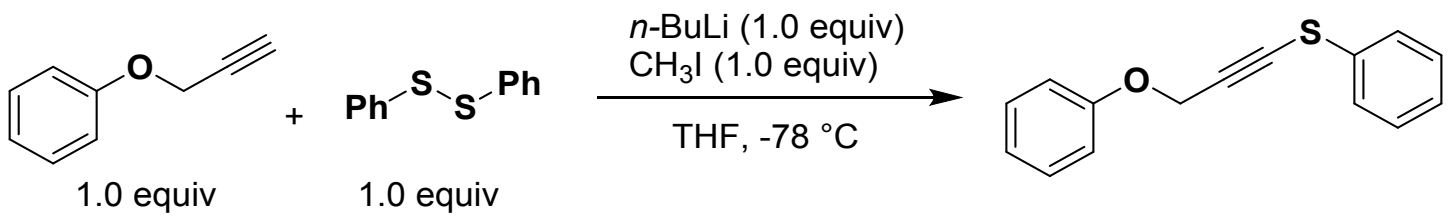

This compound was synthesized following a reported procedure. ${ }^{[9]}$ A solution of phenylpropargyl ether $(0.2643 \mathrm{~g}, 2 \mathrm{mmol})$ in THF $(5 \mathrm{~mL})$ was cooled to $-78^{\circ} \mathrm{C}, n$-BuLi $(1.6$ $\mathrm{M}, 1.25 \mathrm{~mL}, 2 \mathrm{mmol}$ ) was added and the mixture was stirred for $30 \mathrm{~min}$. A premixed solution $(1 \mathrm{~h}, \mathrm{rt})$ of diphenyl sulfide $(0.4366 \mathrm{~g}, 2 \mathrm{mmol})$ and methyl iodide $(0.12 \mathrm{~mL}, 2 \mathrm{mmol})$ in THF $(8 \mathrm{~mL})$ was added dropwise and stirred at room temperature for $1 \mathrm{~h}$. The reaction was quenched with a saturated solution of $\mathrm{NH}_{4} \mathrm{Cl}(20 \mathrm{~mL})$ and extracted with EtOAc $(2 \times 20 \mathrm{~mL})$. The organic phase was dried over $\mathrm{Na}_{2} \mathrm{SO}_{4}$ and concentrated under vacuum. The crude mixture was purified by column chromatography on silica gel using hexane as eluent. The corresponding alkyne was obtained as a paleyellow oil in $30 \%$ yield $(0.1447 \mathrm{~g}) .{ }^{1} \mathbf{H}$ NMR (300 MHz, $\left.\mathbf{C D C l}_{3}\right) \delta 7.42-7.28(\mathrm{~m}, 6 \mathrm{H}), 7.27-7.19(\mathrm{~m}, 1 \mathrm{H}), 7.06-6.97(\mathrm{~m}, 3 \mathrm{H}), 4.93$ (s, 2H). ${ }^{13} \mathbf{C}$ NMR (75 MHz, $\left.\mathbf{C D C l}_{3}\right) \delta 157.65,132.21,129.64,129.37,126.90,126.67,121.71$, 115.19, 94.18, 75.12, 56.92. HRMS-DART calculated for $\mathrm{C}_{15} \mathrm{H}_{13} \mathrm{OS}[\mathrm{M}+\mathrm{H}]^{+}: 241.06871$; found: 241.06844. IR (ATR): 3060, 2911, 2853, 2185, 1597, 1585, 1492, 1478, 1456, 1441, $1361,1291,1260,1209,1173,1108,1063,1024,988,883,821,750,686,504,467 \mathrm{~cm}^{-1}$.

\section{Synthesis of 1-iodo-2-((3-(4-methoxyphenyl)prop-2-yn-1-yl)oxy)benzene (18a). ${ }^{[10]}$}

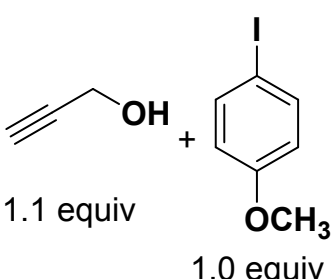

$\mathrm{Pd}\left(\mathrm{PPh}_{3}\right)_{2} \mathrm{Cl}_{2}(1 \mathrm{~mol} \%)$ Cul $(3 \mathrm{~mol} \%)$

$\mathrm{Et}_{3} \mathrm{~N}, \mathrm{rt} .12 \mathrm{~h}$

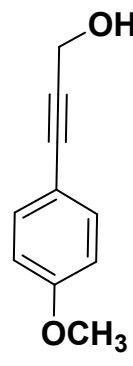

1.05 equiv

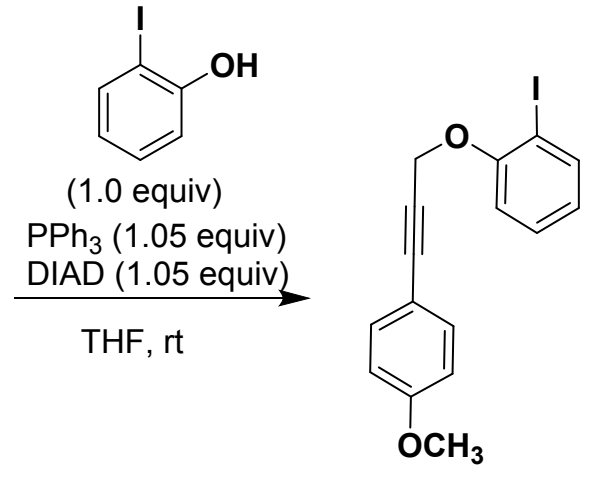


Step I. To a solution of $\mathrm{Pd}\left(\mathrm{PPh}_{3}\right)_{2} \mathrm{Cl}_{2}(35 \mathrm{mg}, 0.01$ equiv, $0.05 \mathrm{mmol})$, $\mathrm{CuI}(19 \mathrm{mg}, 0.03$ equiv, $0.1 \mathrm{mmol})$ and 4-iodoanisole (1.17 g, 1.0 equiv, $5 \mathrm{mmol})$ in $\mathrm{Et}_{3} \mathrm{~N}(20 \mathrm{~mL})$, propargyl alcohol ( $0.32 \mathrm{~mL}, 1.1$ equiv, $5.5 \mathrm{mmol})$ was added and the mixture was stirred overnight at room temperature. Then, it was filtrated throw a pad of celite, washed with EtOAc and concentrated under vacuum. The crude mixture was purified by column chromatography on silica gel using hexane/EtOAc $(2: 1)$ as eluent. The desired product was obtained as a pale yellow solid in $94 \%$ yield $(0.7598 \mathrm{~g})$.

Step II. 3-(4-methoxyphenyl)prop-2-yn-1-ol (0.2554 g, 1.05 equiv, $1.575 \mathrm{mmol})$, 2iodophenol ( $0.33 \mathrm{~g}, 1.0$ equiv, $1.5 \mathrm{mmol})$ and $\mathrm{PPh}_{3}(0.4130 \mathrm{~g}, 1.05$ equiv, $1.575 \mathrm{mmol})$ were dissolved in anhydrous THF $(15 \mathrm{~mL})$ and DIAD $(0.31 \mathrm{~mL}, 1.05$ equiv, $1.575 \mathrm{mmol})$ was added to the mixture at $0{ }^{\circ} \mathrm{C}$. The mixture was stirred at room temperature for $12 \mathrm{~h}$. The solvent was evaporated under vacuum and the crude mixture was purified by column chromatography on silica gel using hexane/EtOAc (98:2) as eluent. The desired compound was obtained as pale yellow oil in 71\% yield $\left.(0.3886 \mathrm{~g}) .{ }^{1} \mathbf{H} \mathbf{~ N M R ~ ( 3 0 0 ~} \mathbf{M H z}, \mathbf{C D C l}_{\mathbf{3}}\right) \delta 7.80$ (dd, $J=7.8,1.6 \mathrm{~Hz}, 1 \mathrm{H}), 7.40-7.34$ (m, 2H), $7.34-7.30$ (m, 1H), $7.10(\mathrm{dd}, J=8.3,1.4 \mathrm{~Hz}$, 1H), $6.86-6.80$ (m, 2H), $6.79-6.72(\mathrm{~m}, 1 \mathrm{H}), 4.98$ (s, 2H), 3.80 (s, 3H). ${ }^{13} \mathbf{C}$ NMR (75 MHz,

$\left.\mathbf{C D C l}_{3}\right) \delta 160.06,156.74,139.75,133.47,129.48,123.36,114.34,114.05,113.46,87.83$, $86.85,82.20,58.08,55.42$.

\section{Synthesis of $N$-(3-(4-methoxyphenyl)prop-2-yn-1-yl)-4-methyl- $N$ phenylbenzenesul- fonamide (21a). ${ }^{[11]}$}<smiles>Nc1ccccc1</smiles>
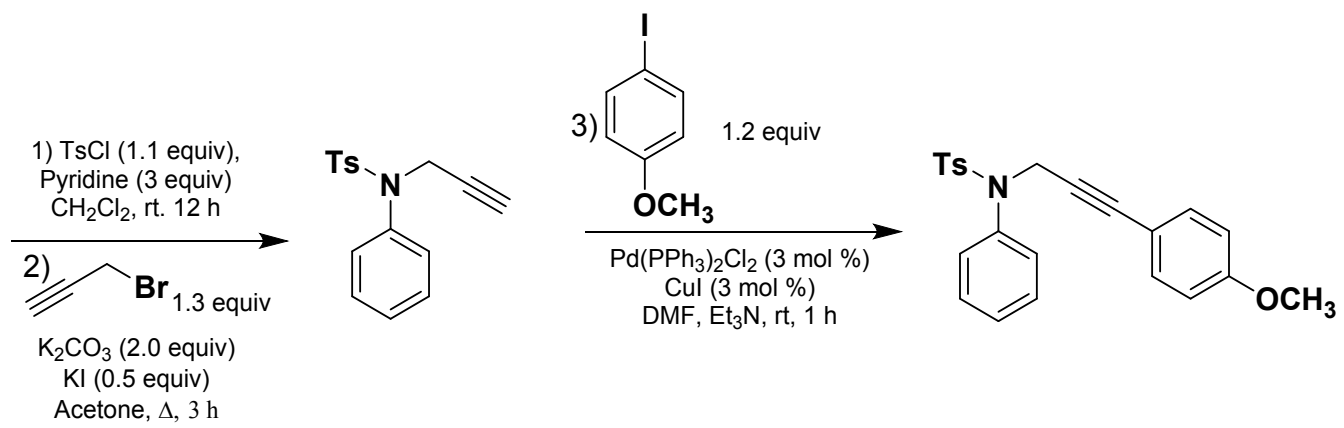

Step I. To a solution of aniline (1.633 g, 1.0 equiv, $17.5 \mathrm{mmol})$ in anhydrous DCM (30 mL), $\mathrm{TsCl}$ (3.6584 g, 1.1 equiv, $19.2 \mathrm{mmol}$ ) was added and the solution cooled to $0{ }^{\circ} \mathrm{C}$. Then, pyridine $(4.24 \mathrm{~mL}, 3.0$ equiv, $52.6 \mathrm{mmol})$ was added dropwise. The reaction mixture was 
allowed to stir at room temperature for $16 \mathrm{~h}$, thereafter was quenched by addition of water $(30 \mathrm{~mL})$ and extracted with DCM $(2 \times 20 \mathrm{~mL})$. The organic layer was washed with $\mathrm{HCl} 1 \mathrm{M}$ solution $(50 \mathrm{~mL})$ and brine $(30 \mathrm{~mL})$, dried over $\mathrm{Na}_{2} \mathrm{SO}_{4}$ and concentrated under vacuum. The desired product was obtained as a pale yellow solid in 91\% yield (3.937 g), and used without further purification in the next reaction.

Step II. To a solution of the $N$-phenylaniline $(0.9682,1.0$ equiv, $3.9 \mathrm{mmol})$ in acetone, propargyl bromide $(0.56 \mathrm{~mL}, 1.3$ equiv, $5.0 \mathrm{mmol})$, KI (0.3249 g, 0.5 equiv, $1.95 \mathrm{mmol})$ and $\mathrm{K}_{2} \mathrm{CO}_{3}$ (1.0821 g, 2.0 equiv, $\left.7.8 \mathrm{mmol}\right)$ were added. The reaction was heated under reflux for $3 \mathrm{~h}$, then water was added, and the mixture extracted with EtOAc. The organic layer was dried over $\mathrm{Na}_{2} \mathrm{SO}_{4}$ and concentrated under vacuum. The residue obtained was purified by column chromatography on silica gel using hexane/EtOAc (8:2) as eluent. The desired compound was obtained as a white solid in $84 \%$ yield $(0.9369 \mathrm{~g})$.

Step III. To a solution of phenylpropargyl sulfonamide, (0.5014 g, 1.0 equiv, $1.75 \mathrm{mmol})$, $\mathrm{Pd}\left(\mathrm{PPh}_{3}\right) \mathrm{Cl}_{2}(37 \mathrm{mg}, 0.01$ equiv, $0.05 \mathrm{mmol})$ and 4-iodoanisole (0.4934 g, 1.2 equiv, 2.1 mmol) in $\mathrm{Et}_{3} \mathrm{~N}(5 \mathrm{~mL})$ was added $\mathrm{CuI}(10 \mathrm{mg}, 0.03$ equiv, $0.05 \mathrm{mmol})$; the mixture was stirred at room temperature for $1 \mathrm{~h}$. Thereafter it was washed with water $(30 \mathrm{~mL})$ and extracted with chloroform $(3 \times 30 \mathrm{~mL})$. The organic layer was washed with brine $(30 \mathrm{~mL})$, dried over $\mathrm{Na}_{2} \mathrm{SO}_{4}$ and concentrated under vacuum. The crude mixture was purified by column chromatography on silica gel using hexane/EtOAc (8:2) as eluent. The desired compound was obtained as a white solid in 69\% yield $(0.4731 \mathrm{~g})$. ${ }^{1} \mathrm{H}$ NMR (400 MHz, $\left.\mathbf{C D C l}_{3}\right) \delta 7.59(\mathrm{~d}, J=8.0 \mathrm{~Hz}, 2 \mathrm{H}), 7.32(\mathrm{~m}, 5 \mathrm{H}), 7.18(\mathrm{~d}, J=8.0 \mathrm{~Hz}, 2 \mathrm{H}), 7.14-7.09(\mathrm{~m}$, 2H), $6.81-6.76(\mathrm{~m}, 2 \mathrm{H}), 4.65(\mathrm{~s}, 2 \mathrm{H}), 3.79(\mathrm{~s}, 3 \mathrm{H}), 2.37$ (s, 3H). ${ }^{13} \mathrm{C}$ NMR (100 MHz,

$\left.\mathbf{C D C l}_{3}\right) \delta 159.81,143.57,139.97,136.12,133.07,129.34,129.09,128.62,128.23,128.18$, 114.57, 113.93, 85.67, 82.31, 55.42, 42.27, 21.66.

\section{Synthesis of aryldiazonium salts. ${ }^{[12]}$}

General procedure: To a solution of the corresponding aniline (1.0 equiv) in anhydrous THF, was added $\mathrm{BF}_{3}-\mathrm{Et}_{2} \mathrm{O}$ (1.4 equiv) at $0{ }^{\circ} \mathrm{C}$ and the mixture was stirred for $30 \mathrm{~min}$. Then, tert-butylnitrite (1.2 equiv) was added dropwise and the reaction mixture was allowed to warm to room temperature over $1 \mathrm{~h}$. Thereafter the solvent was removed under reduced pressure and the resulting solid was washed with cold methanol followed by diethyl ether, 
and filtrated via cannula. All aryldiazonium salts were dried in the high pump vacuum and stored in the dark at $-20^{\circ} \mathrm{C}$.

\section{4-Nitrobenzenediazonium tetrafluoroborate (1b). ${ }^{[13]}$}

$\mathbf{N}_{\mathbf{2}} \mathbf{B F}_{\mathbf{4}}$ Prepared according to the general procedure using 4-nitroaniline $(2.01 \mathrm{~g}, 14.6$<smiles>CC(C)(C)Cc1ccc([N+](=O)[O-])cc1</smiles>
$\mathrm{mmol}), \mathrm{BF}_{3}-\mathrm{Et}_{2} \mathrm{O}(2.5 \mathrm{~mL}, 20.2 \mathrm{mmol})$, tert-butylnitrite $(2 \mathrm{~mL}, 15.1 \mathrm{mmol})$ and THF $(30 \mathrm{~mL})$. The product was obtained as a pale yellow solid $(2.79 \mathrm{~g}, 80 \%) .{ }^{1} \mathbf{H}$ NMR (300 MHz, DMSO- $\left.\boldsymbol{d}_{6}\right) \delta 8.98-8.88(\mathrm{~m}, 2 \mathrm{H}), 8.76-8.67(\mathrm{~m}, 2 \mathrm{H})$.

\section{4-Chlorobenzenediazonium tetrafluoroborate (2b). ${ }^{[13]}$}
$\mathbf{N}_{\mathbf{2}} \mathbf{B F}_{\mathbf{4}}$ Prepared according to the general procedure using 4-chloroaniline $(0.5 \mathrm{~g}, 3.91$ mmol), $\mathrm{BF}_{3}-\mathrm{Et}_{2} \mathrm{O}(0.67 \mathrm{~mL}, 5.4 \mathrm{mmol})$, tert-butylnitrite $(0.62 \mathrm{~mL}, 4.7 \mathrm{mmol})$ and Cl THF $(15 \mathrm{~mL})$. The product was obtained as a beige solid $(0.8877 \mathrm{~g}, 79 \%) .{ }^{1} \mathbf{H}$ NMR (300 MHz, DMSO- $\left.\boldsymbol{d}_{\mathbf{6}}\right) \delta 8.72-8.64(\mathrm{~m}, 2 \mathrm{H}), 8.18-7.98(\mathrm{~m}, 2 \mathrm{H})$.

\section{4-Bromobenzenediazonium tetrafluoroborate (3b). ${ }^{[13]}$}

mmol), $\mathrm{BF}_{3}-\mathrm{Et}_{2} \mathrm{O}(0.5 \mathrm{~mL}, 4.05 \mathrm{mmol})$, tert-butylnitrite $(0.46 \mathrm{~mL}, 3.5 \mathrm{mmol})$ and
THF $(15 \mathrm{~mL})$. The product was obtained as an off-white solid $(0.4254 \mathrm{~g}, 54 \%)$.
${ }^{1} \mathbf{H}$ NMR (300 MHz, DMSO-d $) \delta 8.61-8.49(\mathrm{~m}, 2 \mathrm{H}), 8.31-8.20(\mathrm{~m}, 2 \mathrm{H})$.

\section{4-Fluorobenzenediazonium tetrafluoroborate $(4 \mathbf{b}){ }^{[13]}$}
$\mathbf{N}_{2} \mathbf{B F}_{4}$ Prepared according to the general procedure using 4-fluoroaniline $(0.6371 \mathrm{~g}, 5.73$<smiles>Fc1ccc(I)cc1</smiles> $\mathrm{mmol}), \mathrm{BF}_{3}-\mathrm{Et}_{2} \mathrm{O}(1 \mathrm{~mL}, 8.1 \mathrm{mmol})$, tert-butylnitrite $(0.9 \mathrm{~mL}, 6.8 \mathrm{mmol})$ and THF $(15 \mathrm{~mL})$. The product was obtained as a light-yellow solid $(1.015 \mathrm{~g}, 84 \%) .{ }^{1} \mathbf{H}$ NMR (300 MHz, DMSO-d $\left.\boldsymbol{d}_{6}\right) \delta 8.85$ - $8.74(\mathrm{~m}, 2 \mathrm{H}), 7.97-7.81(\mathrm{~m}, 2 \mathrm{H})$.

\section{4-Ethoxycarbonylbenzenediazonium tetrafluoroborate (5b). ${ }^{[14]}$}

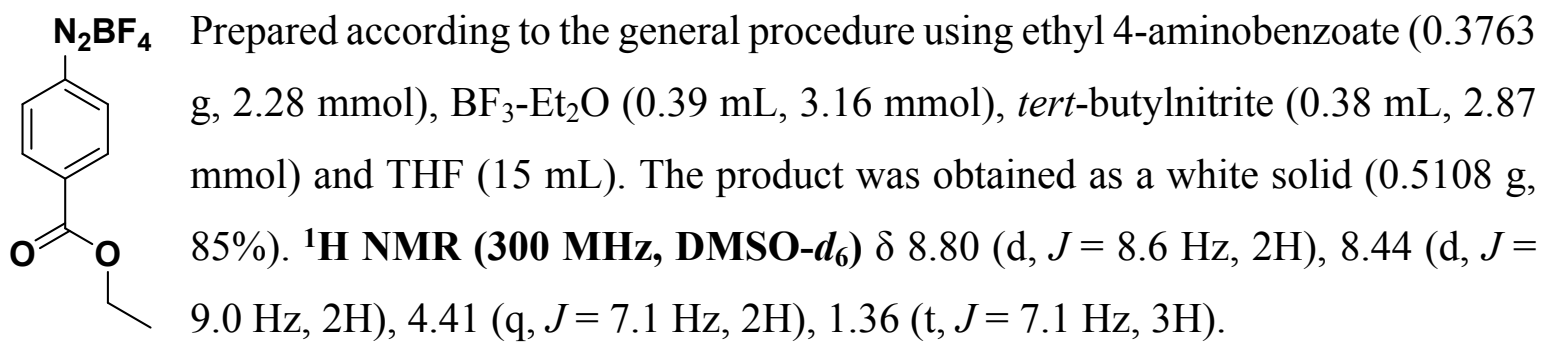




\section{4-Benzoylbenzenediazonium tetrafluoroborate (6b). ${ }^{[15]}$}

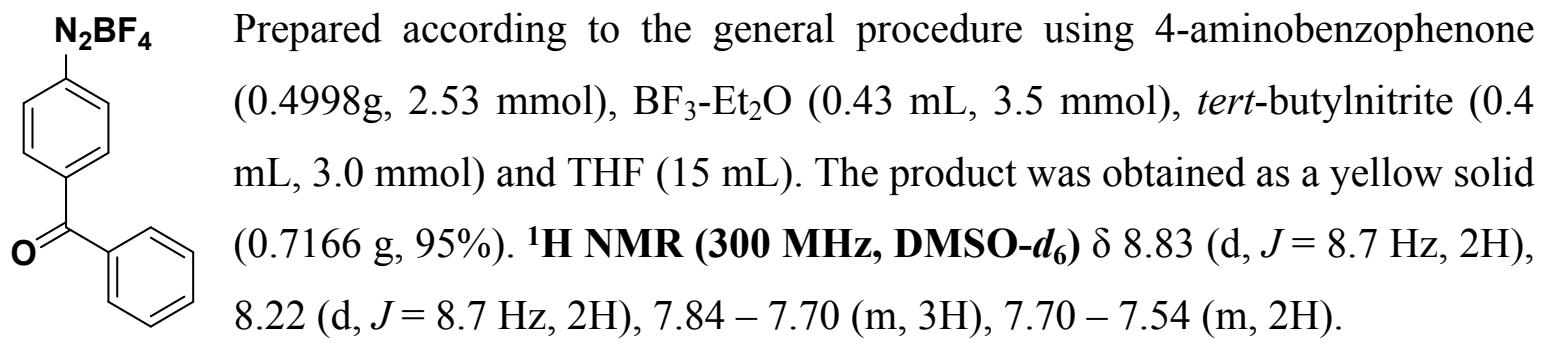

\section{4-Cyanobenzenediazonium tetrafluoroborate (7b). ${ }^{[14]}$}

$4.26 \mathrm{mmol}), \mathrm{BF}_{3}-\mathrm{Et}_{2} \mathrm{O}(0.73 \mathrm{~mL}, 5.91 \mathrm{mmol})$, tert-butylnitrite $(0.67 \mathrm{~mL}, 5.07$
$\mathrm{mmol})$ and $\mathrm{THF}(15 \mathrm{~mL})$. The product was obtained as an off-white solid $(0.6878$
$\mathrm{g}, 74 \%) .{ }^{\mathbf{1}} \mathbf{H} \mathbf{N M R}\left(\mathbf{3 0 0} \mathbf{M H z}, \mathbf{D M S O}-\boldsymbol{d}_{\mathbf{6}}\right) \delta 8.91-8.75(\mathrm{~m}, 2 \mathrm{H}), 8.53-8.35(\mathrm{~m}$, $2 \mathrm{H})$.

\section{3-Nitrobenzenediazonium tetrafluoroborate $(8 b) .{ }^{[16]}$}

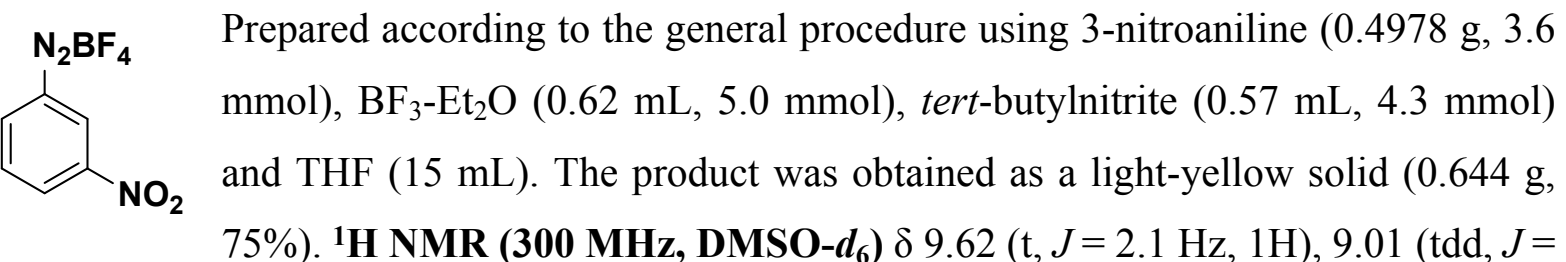
$8.7,2.1,1.0 \mathrm{~Hz}, 2 \mathrm{H}), 8.24(\mathrm{t}, J=8.4 \mathrm{~Hz}, 1 \mathrm{H})$.

\section{3-Cyanobenzenediazonium tetrafluoroborate (9b).}<smiles></smiles>

Prepared according to the general procedure using 3-aminobenzonitrile $(0.5026$ $\mathrm{g}, 4.25 \mathrm{mmol}), \mathrm{BF}_{3}-\mathrm{Et}_{2} \mathrm{O}(0.73 \mathrm{~mL}, 5.9 \mathrm{mmol})$, tert-butylnitrite $(0.67 \mathrm{~mL}, 5.01$ $\mathrm{mmol})$ and THF $(15 \mathrm{~mL})$. The product was obtained as an off-white solid (0.7834 g, 85\%). 1'H NMR (300 MHz, DMSO-d $\left.{ }_{6}\right) \delta 9.22(\mathrm{t}, J=2.0 \mathrm{~Hz}, 1 \mathrm{H})$, $8.94(\mathrm{ddd}, J=8.5,2.1,1.1 \mathrm{~Hz}, 1 \mathrm{H}), 8.71(\mathrm{ddd}, J=8.0,1.6,1.1 \mathrm{~Hz}, 1 \mathrm{H}), 8.24-8.12$ (m, $1 \mathrm{H})$.

\section{3-Chlorobenzenediazonium tetrafluoroborate (10b). ${ }^{[13]}$}




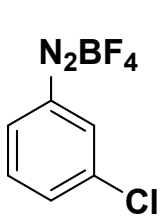

Prepared according to the general procedure A using 3-chloroaniline (0.5g, 3.91 $\mathrm{mmol}), \mathrm{BF}_{3}-\mathrm{Et}_{2} \mathrm{O}(0.67 \mathrm{~mL}, 5.4 \mathrm{mmol})$, tert-butylnitrite $(0.62 \mathrm{~mL}, 4.7 \mathrm{mmol})$ and THF $(15 \mathrm{~mL})$. The product was obtained as a pink solid $(0.8013 \mathrm{~g}, 90 \%)$.

\section{2-Nitrobenzenediazonium tetrafluoroborate (11b). ${ }^{[17]}$}

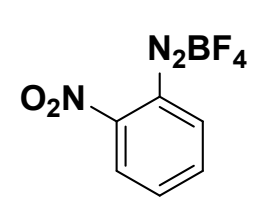

Prepared according to the general procedure using 2-nitroaniline $(0.5031 \mathrm{~g}$, $(0.560 \mathrm{~g}, 65 \%)$.

\section{Gold catalyzed arylative carbocyclization of alkynes.}

\section{General procedure for the synthesis of $2-\mathrm{H}$-chromene derivatives.}

A schlenk tube was charged with $\mathrm{Ph}_{3} \mathrm{PAuCl},(10 \mathrm{~mol} \%)$, the corresponding aryldiazonium salt (1.5-3.5 equiv) and the corresponding alkyne (1.0 equiv). It was evacuated and back filled with nitrogen (x3). Acetonitrile (previously degassed by Freeze-Pump-Thaw method for three cycles) was added to obtain a $0.1 \mathrm{M}$ solution. Then, a freshly prepared solution of ascorbic acid $0.05 \mathrm{M}$ in dry DMSO ( 0.005 equiv) was added followed by DTBPy (1.0 equiv). The reaction mixture was stirred at room temperature for $16-24 \mathrm{~h}$ (or heated at $50^{\circ} \mathrm{C}$ ). After completion by TLC analysis, the solvent was removed under reduced pressure and the crude mixture was purified by column chromatography on silica gel using hexane/EtOAc or hexane $/ \mathrm{Et}_{2} \mathrm{O}$ as eluent.

\section{4-Methyl-3-(4-nitrophenyl)-2H-chromene (1c).}

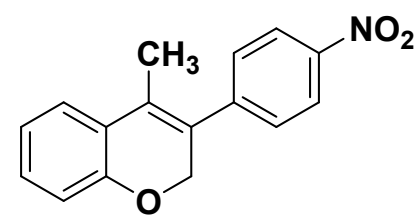

Prepared according to the general procedure using (but-2-yn-1yloxy)benzene (29.5 mg, $0.2 \mathrm{mmol}), \mathrm{Ph}_{3} \mathrm{PAuCl}(10 \mathrm{mg}, 0.02$ $\mathrm{mmol})$, 4-nitrobenzendiazonium tetrafluoroborate $(71.8 \mathrm{mg}, 1.5$ equiv, $0.3 \mathrm{mmol})$, ascorbic acid $(20 \mu \mathrm{L}, 0.01 \mathrm{mmol})$, DTBPy $(45.4 \mu \mathrm{L}, 0.2 \mathrm{mmol})$ and acetonitrile $(3 \mathrm{~mL})$. Purification by column chromatography (silica gel, hexane/EtOAc 98:2) gave the product (34.6 mg, 64\%) as a yellow solid; m.p. 180-182 ${ }^{\circ} \mathrm{C} .{ }^{1} \mathbf{H}$ NMR (300 MHz, $\left.\mathbf{C D C l}_{3}\right) \delta 8.31-8.24(\mathrm{~m}, 2 \mathrm{H}), 7.50-7.42(\mathrm{~m}, 2 \mathrm{H}), 7.32(\mathrm{dd}, J=$ 7.7, $1.6 \mathrm{~Hz}, 1 \mathrm{H}), 7.22(\mathrm{td}, J=7.8,1.6 \mathrm{~Hz}, 1 \mathrm{H}), 7.05-6.97$ (m, 1H), 6.91 (dd, $J=8.0,1.1$ 
$\mathrm{Hz}, 1 \mathrm{H}), 4.89$ (q, $J=1.5 \mathrm{~Hz}, 2 \mathrm{H}), 2.05(\mathrm{t}, J=1.6 \mathrm{~Hz}, 3 \mathrm{H}) .{ }^{13} \mathbf{C}$ NMR (75 MHz, CDCl $\left.\mathbf{3}\right) \delta$ $153.98,147.00,145.53,129.90,129.73,128.40,128.17,124.82,124.54,123.96,121.89$, 116.11, 68.89, 14.69. HRMS-DART calculated for $\mathrm{C}_{16} \mathrm{H}_{14} \mathrm{NO}_{3}[\mathrm{M}+\mathrm{H}]^{+}:$268.09737; found: 268.09771. IR (ATR): 3073, 2961, 2922, 2850, 2815, 1625, 1591, 1509, 1447, 1341, 1261, $1214,1109,1026,1008,978,950,856,827,753,691,565,526,428 \mathrm{~cm}^{-1}$.

\section{4-(4-Methoxyphenyl)-3-(4-nitrophenyl)-2H-chromene (2c).}

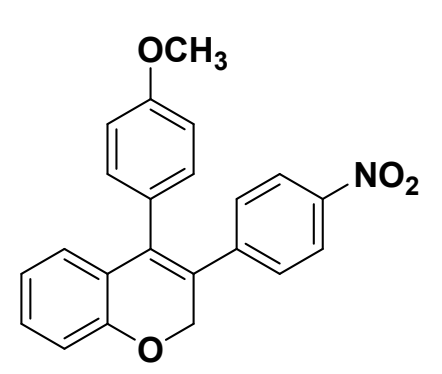

Prepared according to the general procedure using 1-methoxy-4(3-phenoxyprop-1-yn-1-yl)benzene (24.1 $\mathrm{mg}, \quad 0.1 \mathrm{mmol})$, $\mathrm{Ph}_{3} \mathrm{PAuCl} \quad(5 \mathrm{mg}, \quad 0.01 \mathrm{mmol}), \quad$ 4-nitrobenzendiazonium tetrafluoroborate $(35.9 \mathrm{mg}, 1.5$ equiv, $0.15 \mathrm{mmol})$, ascorbic acid (10 $\mu \mathrm{L}, 0.005 \mathrm{mmol})$, DTBPy $(22.7 \mu \mathrm{L}, 0.1 \mathrm{mmol})$ and acetonitrile $(1.5 \mathrm{~mL})$. Purification by column chromatography (silica gel, hexane/EtOAc 95:5) gave the product (29.1 mg, 80\%) as a yellow solid. ${ }^{1} \mathbf{H}$ NMR (300 MHz, $\left.\mathbf{C D C l}_{3}\right) \delta 8.05-7.97(\mathrm{~m}, 2 \mathrm{H}), 7.21(\mathrm{ddd}, J=8.1,5.9,3.1 \mathrm{~Hz}, 1 \mathrm{H}), 7.16-7.09$ $(\mathrm{m}, 2 \mathrm{H}), 7.04-6.98(\mathrm{~m}, 2 \mathrm{H}), 6.97-6.93(\mathrm{~m}, 1 \mathrm{H}), 6.91-6.86(\mathrm{~m}, 2 \mathrm{H}), 6.86-6.80(\mathrm{~m}, 2 \mathrm{H})$, 5.09 (s, 2H), 3.81 (s, 3H). ${ }^{13} \mathbf{C}$ NMR (75 MHz, $\left.\mathbf{C D C l}_{3}\right) \delta$ 159.39, 154.27, 146.21, 145.44, $135.75,131.80,130.07,129.05,128.16,127.20,126.71,125.15,123.47,121.78,116.12$, 114.18, 69.04, 55.33. HRMS-DART calculated for $\mathrm{C}_{22} \mathrm{H}_{18} \mathrm{NO}_{4}[\mathrm{M}+\mathrm{H}]^{+}:$360.12358; found: 360.12190. IR (ATR): 2930, 2850, 1589, 1508, 1343, $1244 \mathrm{~cm}^{-1}$.

\section{4-Butyl-3-(4-nitrophenyl)-2H-chromene (3c).}

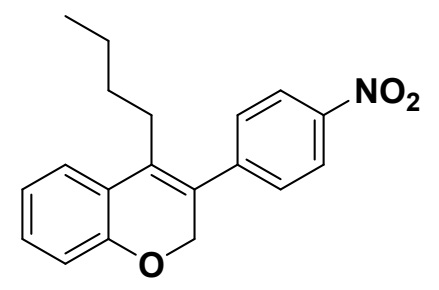

Prepared according to the general procedure using (hept-2-yn-1yloxy)benzene (38.5 mg, $0.2 \mathrm{mmol}), \mathrm{Ph}_{3} \mathrm{PAuCl}(10 \mathrm{mg}, 0.02$ mmol), 4-nitrobenzendiazonium tetrafluoroborate $(71.8 \mathrm{mg}, 1.5$ equiv, $0.3 \mathrm{mmol})$, ascorbic acid (20 $\mu \mathrm{L}, 0.01 \mathrm{mmol})$, DTBPy (45.4 $\mu \mathrm{L}, 0.2 \mathrm{mmol})$ and acetonitrile $(3 \mathrm{~mL})$. Purification by column chromatography (silica gel, hexanes/EtOAc 95:5) gave the product (37 $\mathrm{mg}, 58 \%$ ) as a yellow solid; m.p. $71-73{ }^{\circ} \mathrm{C} .{ }^{1} \mathbf{H}$ NMR (300 $\left.\mathbf{M H z}, \mathbf{C D C l}_{3}\right) \delta 8.31$ - $8.23(\mathrm{~m}, 2 \mathrm{H}), 7.47-7.40$ (m, 2H), $7.32(\mathrm{dd}, J=7.7,1.5 \mathrm{~Hz}, 1 \mathrm{H}), 7.21(\mathrm{ddd}, J=8.0,7.5,1.6 \mathrm{~Hz}, 1 \mathrm{H}), 7.00(\mathrm{td}, J=7.6,1.3$ $\mathrm{Hz}, 1 \mathrm{H}), 6.91(\mathrm{dd}, J=8.0,1.1 \mathrm{~Hz}, 1 \mathrm{H}), 4.84$ (s, 2H), $2.50-2.39$ (m, 2H), $1.51-1.37$ (m, 2H), $1.25(\mathrm{~h}, J=7.2 \mathrm{~Hz}, 3 \mathrm{H}), 0.80$ (t, $J=7.3 \mathrm{~Hz}, 3 \mathrm{H}) .{ }^{13} \mathbf{C} \mathbf{N M R}\left(75 \mathbf{M H z}, \mathbf{C D C l}_{3}\right) \delta 154.51$, 
$147.06,145.85,132.81,129.60,129.50,128.39,124.56,124.05,123.39,121.80,116.47$, 69.05, 31.48, 27.46, 22.73, 13.87. HRMS-DART calculated for $\mathrm{C}_{19} \mathrm{H}_{20} \mathrm{NO}_{3}[\mathrm{M}+\mathrm{H}]^{+}$: 310.14432; found: 310.14347. IR (ATR): 3073, 3033, 2959, 2922, 2861, 1615, 1587, 1506, $1488,1467,1448,1342,1246,1216,1190,1109,1076,1058,1015,928,851,792,754,696$, $522 \mathrm{~cm}^{-1}$.

\section{3-(4-Nitrophenyl)-4-phenyl-2H-chromene (4c).}

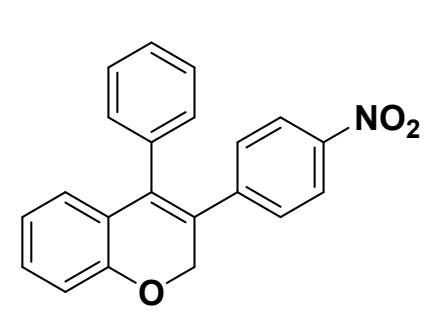

Prepared according to the general procedure using (3phenoxyprop-1-yn-1-yl)benzene ( $42.1 \mathrm{mg}, 0.2 \mathrm{mmol}$ ), $\mathrm{Ph}_{3} \mathrm{PAuCl}$ (10 mg, $0.02 \mathrm{mmol}$ ), 4-nitrobenzendiazonium tetrafluoroborate (71.8 mg, 1.5 equiv, $0.3 \mathrm{mmol}$ ), ascorbic acid ( $20 \mu \mathrm{L}, 0.01 \mathrm{mmol}$ ), DTBPy $(45.4 \mu \mathrm{L}, 0.2 \mathrm{mmol})$ and acetonitrile $(3 \mathrm{~mL})$. Purification by column chromatography (silica gel, hexane/ $\mathrm{Et}_{2} \mathrm{O} 95: 5$ ) gave the product ( $66.5 \mathrm{mg}, 66 \%$ ) as a yellow solid; m.p. $141-144{ }^{\circ} \mathrm{C} .{ }^{\mathbf{1}} \mathbf{H} \mathbf{~ N M R}\left(\mathbf{3 0 0} \mathbf{~ M H z}, \mathbf{C D C l}_{3}\right) \delta 8.00(\mathrm{~d}, J=8.8 \mathrm{~Hz}, 2 \mathrm{H})$, $7.42-7.07$ (m, 8H), 6.97 (d, $J=7.9 \mathrm{~Hz}, 1 \mathrm{H}), 6.91-6.79(\mathrm{~m}, 2 \mathrm{H}), 5.11(\mathrm{~s}, 2 \mathrm{H}) .{ }^{13} \mathbf{C}$ NMR (75 MHz, $\mathbf{C D C l}_{3}$ ) $\delta$ 154.12, 146.32, 145.12, 136.16, 136.03, 130.55, 130.14, 129.07, 128.76, 128.10, 127.17, 127.06, 124.89, 123.44, 121.82, 116.14, 68.99. HRMS-DART calculated for $\mathrm{C}_{21} \mathrm{H}_{15} \mathrm{NO}_{3}[\mathrm{M}+\mathrm{H}]^{+}:$330.11302; found: 330.11332. IR (ATR): 3062, 2957, 2922, 2852, $1618,1587,1508,1482,1448,1441,1339,1249,1214,1106,1036,1003,937,852,835$, $800,764,754,696,585,506 \mathrm{~cm}^{-1}$.

\section{4-(Naphthalen-1-yl)-3-(4-nitrophenyl)-2H-chromene (5c).}

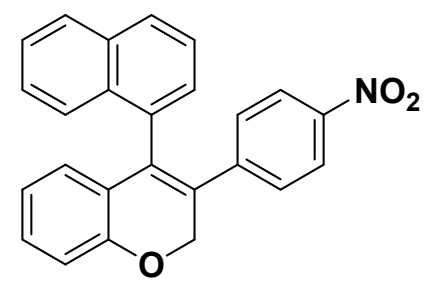

Prepared according to the general procedure using 1-(3phenoxyprop-1-yn-1-yl)naphthalene (26.1 $\mathrm{mg}, \quad 0.1 \mathrm{mmol})$, $\mathrm{Ph}_{3} \mathrm{PAuCl} \quad(5 \mathrm{mg}, \quad 0.01 \mathrm{mmol}), \quad$ 4-nitrobenzendiazonium tetrafluoroborate (35.9 mg, 1.5 equiv, $0.15 \mathrm{mmol})$, ascorbic acid

(10 $\mu \mathrm{L}, 0.005 \mathrm{mmol})$, DTBPy $(22.7 \mu \mathrm{L}, 0.1 \mathrm{mmol})$ and acetonitrile $(1.5 \mathrm{~mL})$. Purification by column chromatography (silica gel, hexane/ $\mathrm{Et}_{2} \mathrm{O} 95: 5$ ) gave the product $(19.5 \mathrm{mg}, 51 \%$ ) as a yellow solid; m.p. $160-163{ }^{\circ} \mathrm{C} .{ }^{1} \mathbf{H}$ NMR (300 MHz, $\left.\mathbf{C D C l}_{3}\right) \delta 7.92-7.79(\mathrm{~m}, 4 \mathrm{H}), 7.74$ (d, $J=8.3 \mathrm{~Hz}, 1 \mathrm{H}), 7.48$ (ddd, $J=8.2,6.9,1.3 \mathrm{~Hz}, 1 \mathrm{H}), 7.44-7.35$ (m, 2H), $7.23-7.15$ (m, 2H), $7.12-7.04$ (m, 2H), 7.01 (dd, $J=8.1,0.9 \mathrm{~Hz}, 1 \mathrm{H}), 6.72$ (td, $J=7.5,1.2 \mathrm{~Hz}, 1 \mathrm{H}), 6.51$ (dd, $J=7.7,1.5 \mathrm{~Hz}, 1 \mathrm{H}), 5.32(\mathrm{~d}, J=13.7 \mathrm{~Hz}, 1 \mathrm{H}), 5.20(\mathrm{~d}, J=13.7 \mathrm{~Hz}, 1 \mathrm{H}) .{ }^{13} \mathbf{C}$ NMR (75 
MHz, $\left.\mathbf{C D C l}_{3}\right) \delta 153.65,146.43,144.81,134.58,133.83,133.65,132.43,130.12,129.13$, $128.73,128.68,128.42,128.23,127.20,126.82,126.32,125.72,124.94,123.40,121.97$, 116.14, 68.99. HRMS-DART calculated for $\mathrm{C}_{25} \mathrm{H}_{18} \mathrm{NO}_{3}[\mathrm{M}+\mathrm{H}]^{+}$: 380.12867; found: 380.12693. IR (ATR): 3102, 3050, 2930, 2840, 1620, 1590, 1509, 1445, 1397, 1339, 1249, $1210,1108,1035,1000,941,853,839,800,766,754,696,589,516 \mathrm{~cm}^{-1}$.

\section{3-(4-Nitrophenyl)-4-(o-tolyl)-2H-chromene (6c).}

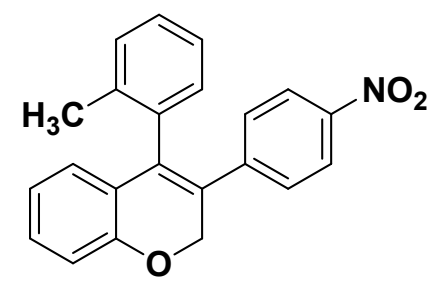

Prepared according to the general procedure using 1-methyl-2-(3phenoxyprop-1-yn-1-yl)benzene ( $20.5 \mathrm{mg}, 0.1 \mathrm{mmol}$ ), $\mathrm{Ph}_{3} \mathrm{PAuCl}$ (5 $\mathrm{mg}, 0.01 \mathrm{mmol}$ ), 4-nitrobenzendiazonium tetrafluoroborate (35.9 mg, 1.5 equiv, $0.15 \mathrm{mmol})$, ascorbic acid $(10 \mu \mathrm{L}, 0.005$ mmol), DTBPy $(22.7 \mu \mathrm{L}, 0.1 \mathrm{mmol})$ and acetonitrile $(3 \mathrm{~mL})$. Purification by column chromatography (silica gel, hexane/ $\mathrm{Et}_{2} \mathrm{O} 95: 5$ ) gave the product (20.5 mg, 59\%) as a yellow solid; m.p. $140-143{ }^{\circ} \mathrm{C} .{ }^{1} \mathbf{H}$ NMR (400 MHz, $\left.\mathbf{C D C l}_{3}\right) \delta 8.02-7.97(\mathrm{~m}, 2 \mathrm{H}), 7.30-7.15$ (m, 4H), $7.15-7.11(\mathrm{~m}, 2 \mathrm{H}), 7.04$ (dd, $J=7.5,1.1 \mathrm{~Hz}, 1 \mathrm{H}), 6.97$ (dd, $J=8.1,1.0 \mathrm{~Hz}, 1 \mathrm{H}), 6.84$ (td, $J=7.6,1.2 \mathrm{~Hz}, 1 \mathrm{H}), 6.64$ (dd, $J=7.7,1.5 \mathrm{~Hz}, 1 \mathrm{H}), 5.16$ (s, 2H), $2.03(\mathrm{~s}, 3 \mathrm{H}) .{ }^{13} \mathbf{C}$ NMR (100 MHz, $\left.\mathbf{C D C l}_{3}\right) \delta 153.90,146.39,144.89,136.79,135.55,130.59,130.13,128.38$, $128.32,127.27,126.72,126.28,124.46,123.45,121.97,116.13,68.77,19.78$. HRMSDART calculated for $\mathrm{C}_{22} \mathrm{H}_{18} \mathrm{NO}_{3}[\mathrm{M}+\mathrm{H}]^{+}: 344.12867$; found: 344.12715. IR (ATR): 3065, 2958, 2923, 2853, 1740, 1626, 1591, 1510, 1450, 1340, 1250, 1215, 1107, 1035, 1021, 851, $838,811,750,726,697,592,513,430 \mathrm{~cm}^{-1}$.

\section{4-(3-Methoxyphenyl)-3-(4-nitrophenyl)-2H-chromene (7c).}

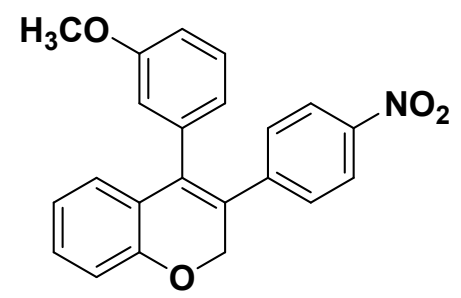

Prepared according to the general procedure using 1-methoxy-

3-(3-phenoxyprop-1-yn-1-yl)benzene (48.2 mg, $0.2 \mathrm{mmol}$ ), $\mathrm{Ph}_{3} \mathrm{PAuCl}$ (10 mg, $0.02 \mathrm{mmol}$ ), 4-nitrobenzendiazonium tetrafluoroborate ( $71.8 \mathrm{mg}, 1.5$ equiv, $0.3 \mathrm{mmol})$, ascorbic acid (20 $\mu \mathrm{L}, 0.01 \mathrm{mmol})$, DTBPy $(45.4 \mu \mathrm{L}, 0.2 \mathrm{mmol})$ and acetonitrile ( $3 \mathrm{~mL})$. Purification by column chromatography (silica gel, hexane/EtOAc 95:5) gave the product (46.2 $\mathrm{mg}, 62 \%)$ as a yellow solid; m.p. $153-155^{\circ} \mathrm{C} .{ }^{1} \mathrm{H}$ NMR $(300 \mathrm{MHz}$, $\left.\mathbf{C D C l}_{3}\right) \delta 8.10-7.93(\mathrm{~m}, 2 \mathrm{H}), 7.26-7.18(\mathrm{~m}, 2 \mathrm{H}), 7.18-7.11(\mathrm{~m}, 2 \mathrm{H}), 6.96(\mathrm{~d}, J=7.9 \mathrm{~Hz}$, 1H), $6.90-6.83$ (m, 3H), $6.72-6.64$ (m, 2H), 5.10 (s, 2H), 3.72 (s, 3H). ${ }^{13}$ C NMR (75 MHz, 
$\left.\mathbf{C D C l}_{3}\right) \delta 159.80,154.05,146.35,145.07,137.51,135.83,130.14,129.85,128.95,127.19$, $126.98,124.76,123.46,122.96,121.85,116.13,116.12,113.45,68.95,55.34$. HRMSDART calculated for $\mathrm{C}_{22} \mathrm{H}_{18} \mathrm{NO}_{4}[\mathrm{M}+\mathrm{H}]^{+}:$360.12358; found: 360.12247. IR (ATR): 3121, 2998, 2690, 2923, 2849, 1621, 1587, 1514, 1482, 1446, 1417, 1341, 1253, 1240, 1152, 1108, $1035,940,844,832,793,753,697,516 \mathrm{~cm}^{-1}$.

\section{3-(4-Nitrophenyl)-4-(thiophen-2-yl)-2H-chromene (9c).}

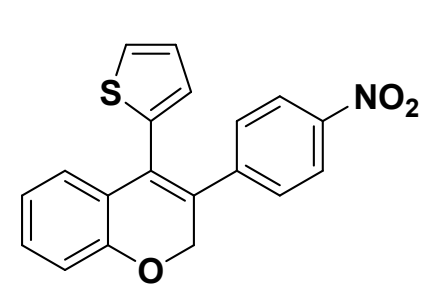

Prepared according to the general procedure using 2-(3phenoxyprop-1-yn-1-yl)thiophene (21.6 $\mathrm{mg}, \quad 0.1 \mathrm{mmol})$, $\mathrm{Ph}_{3} \mathrm{PAuCl} \quad(5 \mathrm{mg}, \quad 0.01 \mathrm{mmol}), \quad$ 4-nitrobenzendiazonium tetrafluoroborate $(35.9 \mathrm{mg}, 1.5$ equiv, $0.15 \mathrm{mmol})$, ascorbic acid $(10 \mu \mathrm{L}, 0.005 \mathrm{mmol})$, DTBPy $(22.7 \mu \mathrm{L}, 0.1 \mathrm{mmol})$ and acetonitrile $(1.5 \mathrm{~mL})$. Purification by column chromatography (silica gel, hexanes/ $\mathrm{Et}_{2} \mathrm{O}$ 95:5) gave the product $(19.5 \mathrm{mg}, 51 \%)$ as a yellow solid; m.p. 124-125 ${ }^{\circ} \mathrm{C} .{ }^{1} \mathbf{H}$ NMR (300 $\left.\mathbf{M H z}, \mathbf{C D C l}_{3}\right) \delta 8.12-8.03(\mathrm{~m}, 2 \mathrm{H}), 7.33$ $(\mathrm{dd}, J=5.1,0.9 \mathrm{~Hz}, 1 \mathrm{H}), 7.27-7.21(\mathrm{~m}, 3 \mathrm{H}), 7.09(\mathrm{dd}, J=7.7,1.6 \mathrm{~Hz}, 1 \mathrm{H}), 7.00$ (dd, $J=$ $5.0,3.4 \mathrm{~Hz}, 1 \mathrm{H}), 6.98-6.88(\mathrm{~m}, 2 \mathrm{H}), 6.86(\mathrm{dd}, J=3.5,0.9 \mathrm{~Hz}, 1 \mathrm{H}), 5.07(\mathrm{~s}, 2 \mathrm{H}) .{ }^{13} \mathbf{C} \mathbf{N M R}$ (75 MHz, $\left.\mathbf{C D C l}_{3}\right) \delta 153.99,146.66,144.97,136.55,130.41,129.85,129.49,129.03,128.91$, $127.35,127.13,126.96,124.75,123.58,122.00,116.16,68.99$. HRMS-DART calculated for $\mathrm{C}_{19} \mathrm{H}_{14} \mathrm{NO}_{3} \mathrm{~S}[\mathrm{M}+\mathrm{H}]^{+}:$336.06944; found: 340.06896. IR (ATR): 3104, 3071, 2957, 2923, 2850, 1590, 1509, 1482, 1450, 1339, 1251, 1215, 822, 754, 696, $521 \mathrm{~cm}^{-1}$.

\section{4-(Benzofuran-2-yl)-3-(4-nitrophenyl)-2H-chromene (10c).}

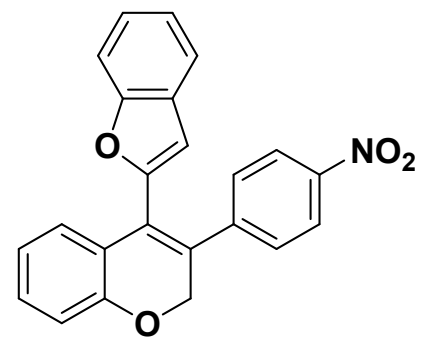

Prepared according to the general procedure using 2-(3phenoxyprop-1-yn-1-yl)benzofuran (50.2 $\mathrm{mg}, \quad 0.2 \mathrm{mmol})$, $\mathrm{Ph}_{3} \mathrm{PAuCl}(10 \mathrm{mg}, 0.02 \mathrm{mmol})$, 4-nitrobenzendiazonium tetrafluoroborate $(95.8 \mathrm{mg}, 2.0$ equiv, $0.4 \mathrm{mmol}$ ), ascorbic acid (20 $\mu \mathrm{L}, 0.01 \mathrm{mmol}), \mathrm{DTBPy}(45.4 \mu \mathrm{L}, 0.2 \mathrm{mmol})$ and acetonitrile (3 mL). Purification by column chromatography (silica gel, hexane/Et ${ }_{2} \mathrm{O}$ 95:5) gave the product (42.2 mg, 56\%) as a yellow solid; m.p. 170-172 ${ }^{\circ} \mathrm{C} .{ }^{1} \mathbf{H}$ NMR (300 MHz, CDCl$)_{3} \delta 8.08(\mathrm{~d}, J=8.8 \mathrm{~Hz}, 2 \mathrm{H}), 7.53(\mathrm{~d}, J=7.7 \mathrm{~Hz}, 1 \mathrm{H}), 7.39$ (d, $J=$ $8.1 \mathrm{~Hz}, 1 \mathrm{H}), 7.34-7.18(\mathrm{~m}, 6 \mathrm{H}), 7.03-6.92(\mathrm{~m}, 2 \mathrm{H}), 6.56(\mathrm{~s}, 1 \mathrm{H}), 5.10(\mathrm{~s}, 2 \mathrm{H}) .{ }^{13} \mathbf{C} \mathbf{N M R}$ (75 MHz, $\left.\mathbf{C D C l}_{3}\right) \delta 154.77,154.11,150.79,147.00,144.71,131.60,130.64,128.62,128.16$, 
$126.80,125.83,125.07,123.75,123.34,122.92,122.19,121.39,116.46,111.53,108.96$, 68.90. HRMS-DART calculated for $\mathrm{C}_{23} \mathrm{H}_{16} \mathrm{NO}_{4}[\mathrm{M}+\mathrm{H}]^{+}: 370.10793$; found: 370.10722 . IR (ATR): 3109, 3081, 2846, 1590, 1510, 1486, 1449, 1341, 1288, 1253, 1216, 1172, 1110, $1002,851,818,750,696,441 \mathrm{~cm}^{-1}$.

\section{3-(4-Nitrophenyl)-4-(phenylthio)-2H-chromene (11c).}

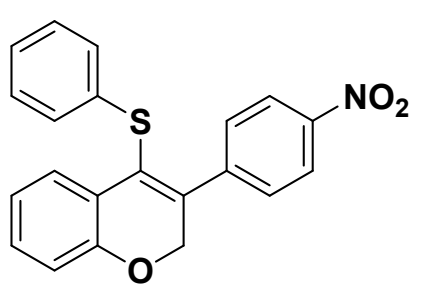

Prepared according to the general procedure using (3phenoxyprop-1-yn-1-yl)(phenyl)sulfide (48.6 mg, $0.2 \mathrm{mmol}$ ),

$\mathrm{Ph}_{3} \mathrm{PAuCl} \quad(10 \quad \mathrm{mg}, \quad 0.02 \mathrm{mmol})$, 4-nitrobenzendiazonium tetrafluoroborate $(71.8 \mathrm{mg}, 1.5$ equiv, $0.3 \mathrm{mmol})$, ascorbic acid ( $20 \mu \mathrm{L}, 0.01 \mathrm{mmol})$, DTBPy $(45.4 \mu \mathrm{L}, 0.2 \mathrm{mmol})$ and acetonitrile $(3 \mathrm{~mL})$. Purification by column chromatography (silica gel, hexane/EtOAc 95:5) gave the product (43.6 mg, $60 \%$ ) as a yellow solid; m.p. $158-160{ }^{\circ} \mathrm{C} .{ }^{1} \mathbf{H}$ NMR $\left(300 \mathbf{~ M H z}, \mathbf{C D C l}_{3}\right) \delta$ $8.26-8.16(\mathrm{~m}, 2 \mathrm{H}), 7.59(\mathrm{dd}, J=7.8,1.5 \mathrm{~Hz}, 1 \mathrm{H}), 7.56-7.48(\mathrm{~m}, 2 \mathrm{H}), 7.23-7.03(\mathrm{~m}, 6 \mathrm{H})$, $6.94(\mathrm{dd}, J=8.1,1.0 \mathrm{~Hz}, 1 \mathrm{H}), 6.87(\mathrm{td}, J=7.7,1.2 \mathrm{~Hz}, 1 \mathrm{H}), 5.05(\mathrm{~s}, 2 \mathrm{H}) .{ }^{13} \mathbf{C}$ NMR (75 MHz, $\left.\mathbf{C D C l}_{3}\right) \delta 154.56,147.44,144.24,139.41,135.78,130.44,129.52,129.25,127.31$, 127.24, 126.53, 125.96, 123.68, 122.50, 122.28, 116.27, 69.75. HRMS-DART calculated for $\mathrm{C}_{21} \mathrm{H}_{16} \mathrm{NO}_{3} \mathrm{~S}[\mathrm{M}+\mathrm{H}]^{+}:$362.08509; found: 362.08544. IR (ATR): 3106, 3069, 2849, 2831, 1593, 1580, 1513, 1475, 1438, 1342, 1261, 1230, 1209, 1148, 1116, 1078, 1024, 1001, 945, $852,824,752,693,588,520,452 \mathrm{~cm}^{-1}$.

\section{6-Methoxy-4-(4-methoxyphenyl)-3-(4-nitrophenyl)-2H-chromene (13c).}

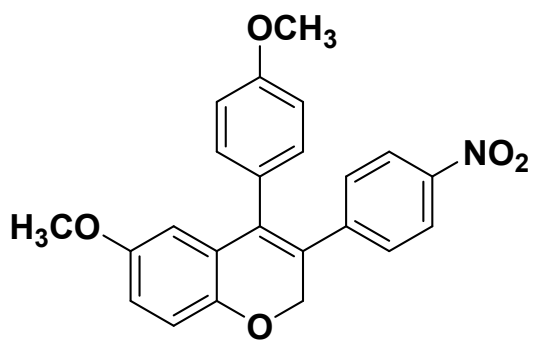

Prepared according to the general procedure using 1methoxy-4-(3-(4-methoxyphenoxy)prop-1-yn-1-yl)benzene (54.2 mg, $0.2 \mathrm{mmol}), \mathrm{Ph}_{3} \mathrm{PAuCl}(10 \mathrm{mg}, 0.02 \mathrm{mmol})$, 4-nitrobenzendiazonium tetrafluoroborate $(71.8 \mathrm{mg}, 1.5$ equiv, $0.3 \mathrm{mmol})$, ascorbic acid $(20 \mu \mathrm{L}, 0.01 \mathrm{mmol})$, DTBPy $(45.4 \mu \mathrm{L}, 0.2 \mathrm{mmol})$ and acetonitrile $(3 \mathrm{~mL})$.

Purification by column chromatography (silica gel, hexane/EtOAc 9:1) gave the product (43.3 mg, 55\%) as an orange solid; m.p. $179-181{ }^{\circ} \mathrm{C} .{ }^{1} \mathbf{H}$ NMR (300 $\left.\mathbf{~ M H z}, \mathbf{C D C l}_{3}\right) \delta 8.06-$ $7.96(\mathrm{~m}, 2 \mathrm{H}), 7.16-7.08(\mathrm{~m}, 2 \mathrm{H}), 7.05-6.96(\mathrm{~m}, 2 \mathrm{H}), 6.90(\mathrm{~d}, J=8.7 \mathrm{~Hz}, 1 \mathrm{H}), 6.86-6.80$ (m, 2H), 6.77 (dd, $J=8.7,3.0 \mathrm{~Hz}, 1 \mathrm{H}), 6.44$ (d, $J=2.9 \mathrm{~Hz}, 1 \mathrm{H}), 5.03(\mathrm{~s}, 2 \mathrm{H}), 3.81(\mathrm{~s}, 3 \mathrm{H})$, 
3.65 (s, 3H). ${ }^{13}$ C NMR (75 MHz, CDCl $_{3}$ ) $\delta$ 159.42, 154.39, 148.27, 146.25, 145.49, 135.77, 131.79, 129.06, 128.08, 127.66, 125.97, 123.46, 116.59, 115.06, 114.21, 112.72, 69.19, 55.83，55.33. HRMS-DART calculated for $\mathrm{C}_{23} \mathrm{H}_{20} \mathrm{NO}_{5}[\mathrm{M}+\mathrm{H}]^{+}$: 390.13415; found: 390.13324. IR (ATR): 3066, 3003, 2948, 2926, 2836, 1608, 1571, 1505, 1486, 1448, 1424, 1336, 1283, 1248, 1204, 1178, 1107, 1062, 1032, 879, 834, 787, 757, 735, 698, 560, 518 $\mathrm{cm}^{-1}$.

6-(Tert-butyl)-4-(4-methoxyphenyl)-3-(4-nitrophenyl)-2H-chromene (14c).

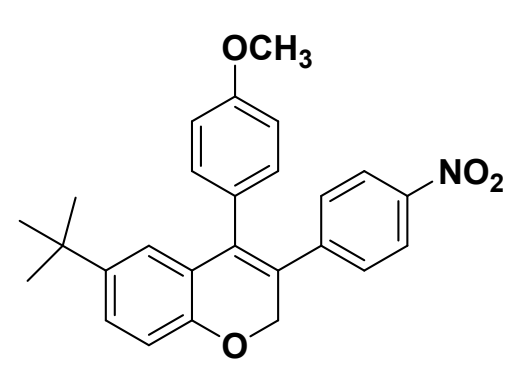

Prepared according to the general procedure using 1-(tertbutyl)-4-((3-(4-methoxyphenyl)prop-2-yn-1-yl)oxy)benzene (59.5 mg, $0.2 \mathrm{mmol}), \mathrm{Ph}_{3} \mathrm{PAuCl}(10 \mathrm{mg}, 0.02 \mathrm{mmol}), 4-$ nitrobenzendiazonium tetrafluoroborate $(71.8 \mathrm{mg}, 1.5$ equiv, $0.3 \mathrm{mmol})$, ascorbic acid (20 $\mu \mathrm{L}, 0.01 \mathrm{mmol})$, DTBPy (45.4 $\mu \mathrm{L}, 0.2 \mathrm{mmol})$ and acetonitrile $(3 \mathrm{~mL})$. Purification by column chromatography (silica gel, hexane/EtOAc 95:5) and recrystallization (DCMhexane) gave the product (47.8 mg, 55\%) as a yellow solid; m.p. $160-162{ }^{\circ} \mathrm{C} .{ }^{1} \mathbf{H}$ NMR (300 MHz, $\left.\mathbf{C D C l}_{3}\right) \delta 8.03(\mathrm{~d}, J=8.6 \mathrm{~Hz}, 2 \mathrm{H}), 7.27(\mathrm{dd}, J=8.3,2.1 \mathrm{~Hz}, 1 \mathrm{H}), 7.12(\mathrm{~d}, J=8.6 \mathrm{~Hz}$, 2H), $7.02(\mathrm{~d}, J=8.5 \mathrm{~Hz}, 2 \mathrm{H}), 6.97-6.82(\mathrm{~m}, 4 \mathrm{H}), 5.07$ (s, 2H), $3.84(\mathrm{~s}, 3 \mathrm{H}), 1.21(\mathrm{~s}, 9 \mathrm{H})$.

${ }^{13}$ C NMR (75 MHz, $\mathbf{C D C l}_{3}$ ) $\delta$ 159.36, 152.08, 146.14, 145.75, 144.49, 136.21, 131.90, 129.07, 128.25, 126.99, 126.46, 124.45, 124.22, 123.45, 115.45, 114.07, 69.11, 55.33, 34.39, 31.48. HRMS-DART calculated for $\mathrm{C}_{26} \mathrm{H}_{26} \mathrm{NO}_{4}[\mathrm{M}+\mathrm{H}]^{+}: 416.18618$; found: 416.18472. IR (ATR): 3066, 3002, 2948, 2926, 2845, 1608, 1589, 1571, 1505, 1486, 1460, 1424, 1336, $1283,1248,1204,1178,1107,1032,1010,879,852,821,757,698,560 \mathrm{~cm}^{-1}$.

\section{6-(Benzyloxy)-4-(4-methoxyphenyl)-3-(4-nitrophenyl)-2H-chromene (15c).}

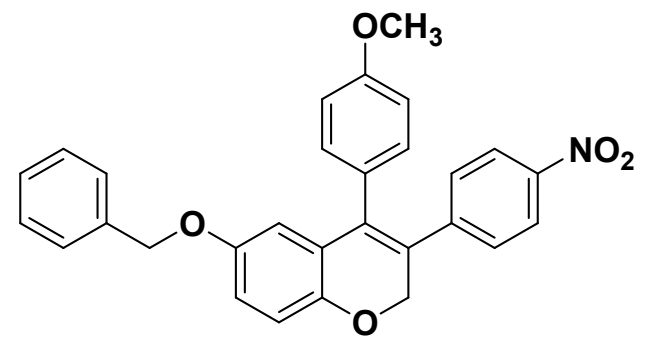

Prepared according to the general procedure using 1(benzyloxy)-4-((3-(4-methoxyphenyl)prop-2-yn-1yl)oxy)benzene (69.6 mg, $0.2 \mathrm{mmol}), \mathrm{Ph}_{3} \mathrm{PAuCl}$ (10 $\mathrm{mg}, \quad 0.02 \mathrm{mmol}), \quad$ 4-nitrobenzendiazonium tetrafluoroborate $(71.8 \mathrm{mg}, 1.5$ equiv, $0.3 \mathrm{mmol})$, ascorbic acid (20 $\mu \mathrm{L}, 0.01 \mathrm{mmol})$, DTBPy $(45.4 \mu \mathrm{L}$,

$0.2 \mathrm{mmol}$ ) and acetonitrile $(3 \mathrm{~mL})$. Purification by column chromatography (silica gel, 
hexane/EtOAc 9:1) gave the product (72.2 mg, 77\%) as a yellow solid; m.p. $123-125^{\circ} \mathrm{C} .{ }^{1} \mathbf{H}$ NMR (300 MHz, CDCl$) \delta 8.05-7.95$ (m, 2H), $7.41-7.24(\mathrm{~m}, 5 \mathrm{H}), 7.14-7.08$ (m, 2H), 6.99-6.93 (m, 2H), $6.90(\mathrm{~d}, J=8.5 \mathrm{~Hz}, 1 \mathrm{H}), 6.86(\mathrm{~d}, J=2.8 \mathrm{~Hz}, 1 \mathrm{H}), 6.84-6.78(\mathrm{~m}, 2 \mathrm{H})$, $6.51(\mathrm{~d}, J=2.7 \mathrm{~Hz}, 1 \mathrm{H}), 5.03(\mathrm{~s}, 2 \mathrm{H}), 4.90$ (s, 2H), 3.82 (s, 3H). ${ }^{13} \mathbf{C}$ NMR (75 MHz, CDCl$)$ $\delta 159.37,153.49,148.42,146.22,145.47,137.04,135.75,131.76,129.05,128.66,128.06$, 128.01, 127.67, 127.59, 123.46, 116.63, 116.29, 114.23, 113.78, 70.70, 69.18, 55.33. HRMS-DART calculated for $\mathrm{C}_{29} \mathrm{H}_{24} \mathrm{NO}_{5}[\mathrm{M}+\mathrm{H}]^{+}$: 466.16545; found: 466.16408. IR (ATR): 3030, 2970, 2934, 2905, 1606, 1592, 1571, 1510, 1486, 1461, 1424, 1342, 1282 , $1245,1199,1173,1108,1023,911,880,852,832,756,727,696,577,564,513 \mathrm{~cm}^{-1}$.

\section{4-(4-Methoxyphenyl)-3-(4-nitrophenyl)-6-phenyl-2H-chromene (16c).}

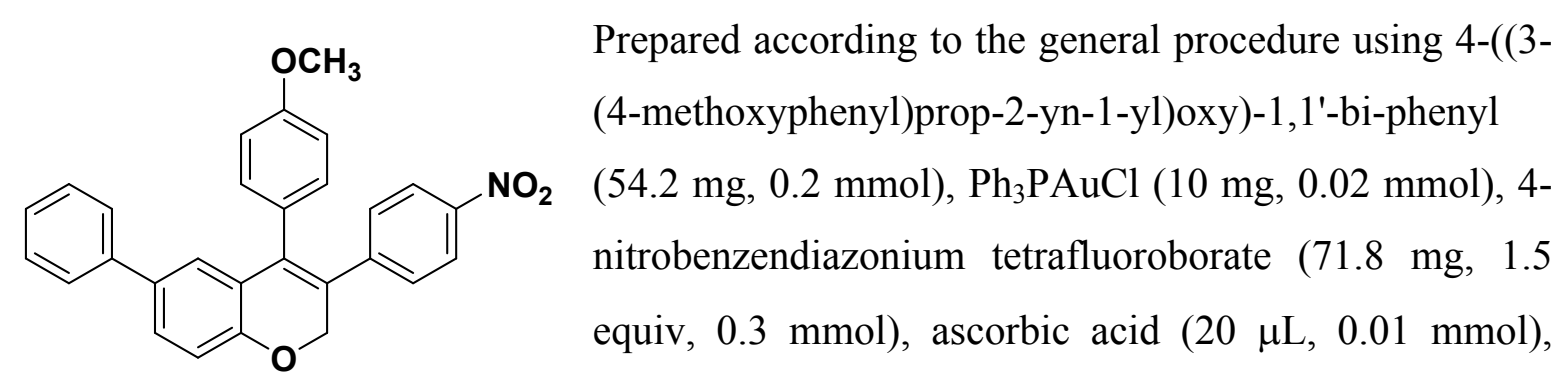

DTBPy $(45.4 \mu \mathrm{L}, 0.2 \mathrm{mmol})$ and acetonitrile $(3 \mathrm{~mL})$. Purification by column chromatography (silica gel, hexane/EtOAc 9:1) gave the product (52.4 mg, 60\%) as a yellow solid; m.p. 154$156{ }^{\circ} \mathrm{C} .{ }^{1} \mathbf{H}$ NMR (300 MHz, $\left.\mathbf{C D C l}_{3}\right) \delta 8.08-7.99(\mathrm{~m}, 2 \mathrm{H}), 7.46(\mathrm{dd}, J=8.3,2.3 \mathrm{~Hz}, 1 \mathrm{H})$, $7.44-7.38(\mathrm{~m}, 3 \mathrm{H}), 7.37-7.33(\mathrm{~m}, 1 \mathrm{H}), 7.32$ - $7.25(\mathrm{~m}, 1 \mathrm{H}), 7.17$ - $7.11(\mathrm{~m}, 3 \mathrm{H}), 7.07$ $7.02(\mathrm{~m}, 3 \mathrm{H}), 6.88-6.81(\mathrm{~m}, 2 \mathrm{H}), 5.13(\mathrm{~s}, 2 \mathrm{H}), 3.82(\mathrm{~s}, 3 \mathrm{H}) \cdot{ }^{13} \mathbf{C}$ NMR (75 MHz, CDCl $) \delta$ $159.43,153.87,146.26,145.42,140.74,135.77,134.97,131.83,129.09,128.84,128.80$, 127.98, 127.04, 127.03, 126.87, 125.79, 125.35, 123.50, 116.49, 114.28, 69.17, 55.33. HRMS-DART calculated for $\mathrm{C}_{28} \mathrm{H}_{22} \mathrm{NO}_{4}[\mathrm{M}+\mathrm{H}]^{+}:$436.15488; found: 436.15696. IR (ATR): 3106, 3069, 2849, 2831, 1593, 1580, 1513, 1475, 1438, 1342, 1261, 1230, 1209, $1148,1116,1078,1024,1001,945,852,824,752,693,588,520,452 \mathrm{~cm}^{-1}$.

\section{6-Bromo-4-(4-methoxyphenyl)-3-(4-nitrophenyl)-2H-chromene (17c).}

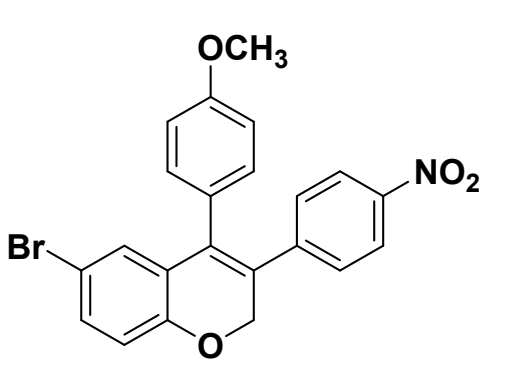

Prepared according to the general procedure using 1-bromo4-((3-(4-methoxyphenyl)prop-2-yn-1-yl)oxy)benzene (64.1 $\mathrm{mg}, 0.2 \mathrm{mmol}), \quad \mathrm{Ph}_{3} \mathrm{PAuCl}(10 \mathrm{mg}, 0.02 \mathrm{mmol})$, 4nitrobenzendiazonium tetrafluoroborate $(95.8 \mathrm{mg}, 2.0$ equiv, 
$0.4 \mathrm{mmol})$, ascorbic acid $(20 \mu \mathrm{L}, 0.01 \mathrm{mmol})$, DTBPy $(45.4 \mu \mathrm{L}, 0.2 \mathrm{mmol})$ and acetonitrile (3 mL). Purification by column chromatography (silica gel, hexane/EtOAc 95:5) gave the product (39 mg, 44\%) as a yellow solid; m.p. 190-192 ${ }^{\circ} \mathrm{C} .{ }^{1} \mathbf{H}$ NMR (300 MHz, $\left.\mathbf{C D C l}_{3}\right) \delta$ $8.02(\mathrm{~d}, J=8.9 \mathrm{~Hz}, 2 \mathrm{H}), 7.29(\mathrm{dd}, J=8.5,2.4 \mathrm{~Hz}, 1 \mathrm{H}), 7.11(\mathrm{~d}, J=8.9 \mathrm{~Hz}, 2 \mathrm{H}), 7.01-6.94$ $(\mathrm{m}, 3 \mathrm{H}), 6.88-7.78(\mathrm{~m}, 3 \mathrm{H}), 5.08(\mathrm{~s}, 2 \mathrm{H}), 3.82(\mathrm{~s}, 3 \mathrm{H}) .{ }^{13} \mathbf{C}$ NMR (75 MHz, CDCl$) \delta$ $159.58,153.28,146.42,144.90,134.72,132.60$, 131.67, 129.58, 129.06, 127.85, 127.32, 127.06, 123.53, 117.94, 114.43, 114.12, 69.07, 55.35. HRMS-DART calculated for $\mathrm{C}_{22} \mathrm{H}_{17} \mathrm{BrNO}_{4}[\mathrm{M}+\mathrm{H}]^{+}:$438.03410; found: 438.03297. IR (ATR): 3069, 3009, 2972, 2931, 2833, 1610, 1585, 1477, 1452, 1403, 1340, 1285, 1245, 1217, 1172, 1109, 1027, 1006, 933, $873,853,825,730,697,555 \mathrm{~cm}^{-1}$.

\section{8-Iodo-4-(4-methoxyphenyl)-3-(4-nitrophenyl)-2H-chromene (18c).}

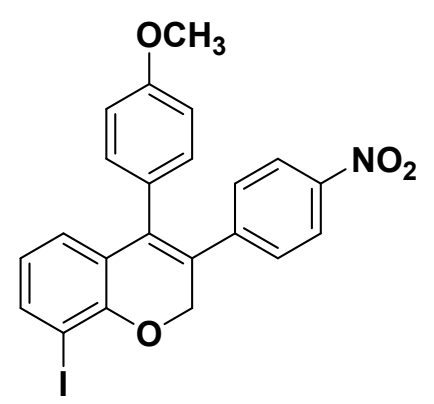

Prepared according to the general procedure using 1-iodo-2-((3(4-methoxyphenyl)prop-2-yn-1-yl)oxy)benzene (73.6 mg, 0.2 $\mathrm{mmol}), \mathrm{Ph}_{3} \mathrm{PAuCl}(10 \mathrm{mg}, 0.02 \mathrm{mmol})$, 4-nitrobenzendiazonium tetrafluoroborate $(95.8 \mathrm{mg}, 2.0$ equiv, $0.4 \mathrm{mmol})$, ascorbic acid (20 $\mu \mathrm{L}, 0.01 \mathrm{mmol}), \mathrm{DTBPy}(45.4 \mu \mathrm{L}, 0.2 \mathrm{mmol})$ and acetonitrile (3 mL). Purification by column chromatography (silica gel, hexanes/EtOAc 95:5) gave the product (73.8 mg, 45\%) as a yellow solid; m.p. $139-142{ }^{\circ} \mathrm{C}$. ${ }^{1} \mathbf{H}$ NMR (300 MHz, $\left.\mathbf{C D C l}_{3}\right) \delta 8.05-7.99(\mathrm{~m}, 2 \mathrm{H}), 7.63(\mathrm{dd}, J=7.8,1.5 \mathrm{~Hz}, 1 \mathrm{H}), 7.15-$ $7.09(\mathrm{~m}, 2 \mathrm{H}), 7.00-6.95(\mathrm{~m}, 2 \mathrm{H}), 6.88-(\mathrm{m}, 3 \mathrm{H}), 6.64(\mathrm{t}, J=7.8 \mathrm{~Hz}, 1 \mathrm{H}), 5.18(\mathrm{~s}, 2 \mathrm{H}), 3.81$ (s, 3H). ${ }^{13} \mathbf{C}$ NMR (75 MHz, $\left.\mathbf{C D C l}_{3}\right) \delta$ 159.53, 153.44, 146.44, 144.90, 139.39, 135.40, $131.75,129.07,127.67,127.46,127.31,125.91,123.58,123.41,114.30,84.08,69.71,55.36$. HRMS-DART calculated for $\mathrm{C}_{22} \mathrm{H}_{17} \mathrm{INO}_{4}[\mathrm{M}+\mathrm{H}]^{+}$: 486.02023; found: 486.02053. IR (ATR): 3068, 3031, 2976, 2934, 2841, 1613, 1590, 1514, 1462, 1435, 1340, 1286, 1249, $1231,1173,1111,1021,857,783,754,692,556,523 \mathrm{~cm}^{-1}$.

\section{1-(4-(4-Methoxyphenyl)-3-(4-nitrophenyl)-2H-chromen-6-yl)ethan-1-one (19c).}

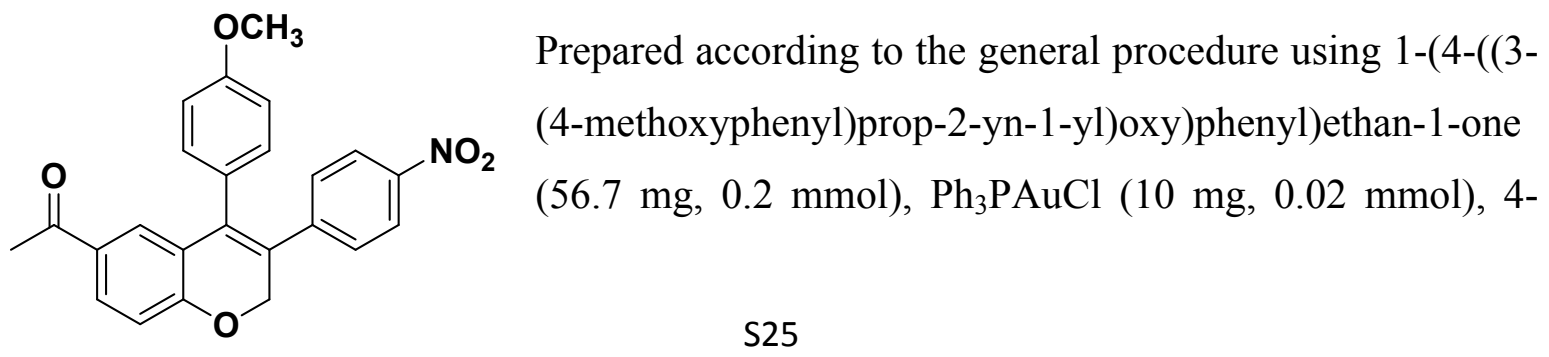


nitrobenzendiazonium tetrafluoroborate ( $119.7 \mathrm{mg}, 2.5$ equiv, $0.5 \mathrm{mmol})$, ascorbic acid (20 $\mu \mathrm{L}, 0.01 \mathrm{mmol})$, DTBPy ( $45.4 \mu \mathrm{L}, 0.2 \mathrm{mmol})$ and acetonitrile $(3 \mathrm{~mL})$. Purification by column chromatography (silica gel, hexane/EtOAc 8:2) gave the product (27.9 $\mathrm{mg}, 34 \%$ ) as a yellow solid; m.p. 177-179 ${ }^{\circ} \mathrm{C} .{ }^{1} \mathbf{H}$ NMR (300 MHz, $\left.\mathbf{C D C l}_{3}\right) \delta 8.06-7.98(\mathrm{~m}, 2 \mathrm{H}), 7.84(\mathrm{dd}, J=$ 8.4, 2.2 Hz, 1H), 7.53 (d, $J=2.1 \mathrm{~Hz}, 1 \mathrm{H}), 7.17-7.10$ (m, 2H), $7.02-6.94$ (m, 3H), $6.87-$ $6.80(\mathrm{~m}, 2 \mathrm{H}), 5.16$ (s, 2H), $3.82(\mathrm{~s}, 3 \mathrm{H}), 2.43(\mathrm{~s}, 3 \mathrm{H}) .{ }^{13} \mathbf{C} \mathbf{~ N M R}\left(75 \mathbf{M H z}, \mathbf{C D C l}_{3}\right) \delta 196.85$, $159.63,158.35,146.47,144.86,135.03,131.69,131.32,130.83,129.12,127.74,127.26$, 127.11, 124.62, 123.57, 116.25, 114.46, 69.27, 55.35, 26.48. HRMS-DART calculated for $\mathrm{C}_{24} \mathrm{H}_{20} \mathrm{NO}_{5}[\mathrm{M}+\mathrm{H}]^{+}:$402.13415; found: 402.13312. IR (ATR): 3073, 3020, 2971, 2934, 2853, 2836, 1677, 1591, 1510, 1491, 1456, 1422, 1343, 1286, 1250, 1238, 1172, 1109, 1025, $1003,944,852,834,807,754,730,699,614,569,511 \mathrm{~cm}^{-1}$.

\section{4-(4-Methoxyphenyl)-3-(4-nitrophenyl)-2H-thiochromene (20c).}

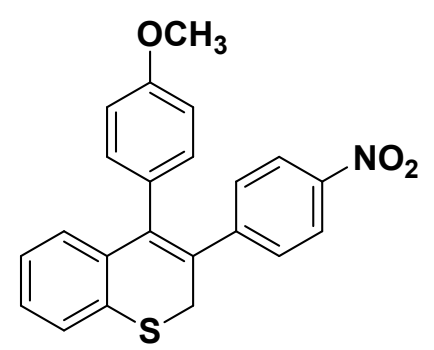

Prepared according to the general procedure using (3-(4methoxyphenyl)prop-2-yn-1-yl)(phenyl)sulfane (53.5 mg, 0.2 $\mathrm{mmol}), \mathrm{Ph}_{3} \mathrm{PAuCl}$ (10 mg, $0.02 \mathrm{mmol}$ ), 4-nitrobenzendiazonium tetrafluoroborate $(71.8 \mathrm{mg}, 1.5$ equiv, $0.3 \mathrm{mmol})$, ascorbic acid (20 $\mu \mathrm{L}, 0.01 \mathrm{mmol})$, DTBPy $(45.4 \mu \mathrm{L}, 0.2 \mathrm{mmol})$ and acetonitrile

(3 $\mathrm{mL}$ ). Purification by column chromatography (silica gel, hexanes/EtOAc 98:2) gave the product (13.8 mg, 18\%) as a yellow solid; m.p. 201-203 ${ }^{\circ} \mathrm{C}$. ${ }^{1}$ H NMR (400 MHz, $\left.\mathbf{C D C l}_{3}\right) \delta 8.03-7.98(\mathrm{~m}, 2 \mathrm{H}), 7.41(\mathrm{dd}, J=7.8,1.1 \mathrm{~Hz}, 1 \mathrm{H}), 7.23-$ $7.18(\mathrm{~m}, 2 \mathrm{H}), 7.16(\mathrm{dd}, J=7.6,1.4 \mathrm{~Hz}, 1 \mathrm{H}), 7.03(\mathrm{td}, J=7.7,1.3 \mathrm{~Hz}, 1 \mathrm{H}), 6.92-6.86(\mathrm{~m}$, 3H), $6.78-6.71$ (m, 2H), 3.78 (s, 3H), 3.76 (s, 2H). ${ }^{13} \mathbf{C}$ NMR (100 MHz, CDCl $)$ o 159.15, 148.97, 145.96, 139.54, 136.18, 133.49, 132.55, 131.19, 129.67, 129.57, 128.87, 128.22, 127.56, 125.60, 123.45, 113.88, 55.31, 31.76. HRMS-DART calculated for $\mathrm{C}_{22} \mathrm{H}_{18} \mathrm{NO}_{3} \mathrm{~S}$ $[\mathrm{M}+\mathrm{H}]^{+}:$376.10074; found: 376.10014. IR (ATR): 2954, 2923, 2853, 2181, 1732, 1601, $1509,1463,1344,1257,1080,1031,967,804,751 \mathrm{~cm}^{-1}$.

\section{4-(4-Methoxyphenyl)-3-(4-nitrophenyl)-1-tosyl-1,2-dihydroquinoline (21c).}

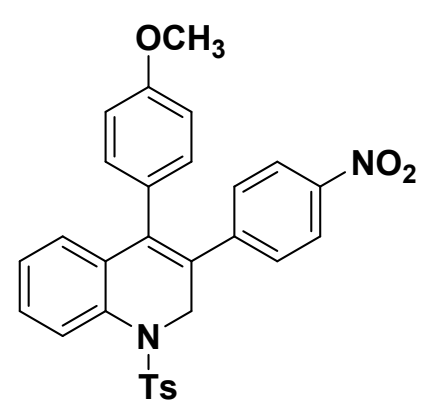

Prepared according to the general procedure using $N$-(3-(4methoxyphenyl)prop-2-yn-1-yl)-4-methyl- $N$-phenylbenzenesulfonamide (79.1 mg, $0.2 \mathrm{mmol}), \mathrm{Ph}_{3} \mathrm{PAuCl}(10 \mathrm{mg}, 0.02 \mathrm{mmol}), 4-$ 
nitrobenzendiazonium tetrafluoroborate ( $167.6 \mathrm{mg}, 3.5$ equiv, $0.7 \mathrm{mmol})$, ascorbic acid (20 $\mu \mathrm{L}, 0.01 \mathrm{mmol})$, DTBPy ( $45.4 \mu \mathrm{L}, 0.2 \mathrm{mmol})$ and acetonitrile $(3 \mathrm{~mL})$. Purification by column chromatography (silica gel, hexane/EtOAc 8:2) gave the product (36.3 $\mathrm{mg}, 35 \%$ ) as a yellow solid; This compound decomposes above $200{ }^{\circ} \mathrm{C}$. ${ }^{1} \mathbf{H}$ NMR (300 $\left.\mathbf{~ M H z}, \mathbf{C D C l}_{3}\right) \delta 8.03-7.95$ (m, 2H), 7.85 (dd, $J=8.0,1.1 \mathrm{~Hz}, 1 \mathrm{H}), 7.38$ (td, $J=7.8,1.5 \mathrm{~Hz}, 1 \mathrm{H}), 7.28-7.07$ (m, 5H), $6.76(\mathrm{td}, J=7.3,1.7 \mathrm{~Hz}, 3 \mathrm{H}), 6.70-6.62(\mathrm{~m}, 2 \mathrm{H}), 6.36-6.28(\mathrm{~m}, 2 \mathrm{H}), 4.83(\mathrm{~s}, 2 \mathrm{H}), 3.77$ (s, 3H), 2.42 (s, 3H). ${ }^{13} \mathbf{C}$ NMR (75 MHz, $\mathbf{C D C l}_{3}$ ) $\delta$ 159.33, 146.21, 145.68, 143.94, 136.75, 136.35, 135.27, 132.32, 131.64, 129.37, 129.11, 128.84, 128.25, 127.98, 127.59, 127.47, 127.34，127.00，123.39，113.93，55.32，50.16，21.64. HRMS-DART calculated for $\mathrm{C}_{29} \mathrm{H}_{25} \mathrm{~N}_{2} \mathrm{O}_{5} \mathrm{~S}[\mathrm{M}+\mathrm{H}]^{+}:$513.14842; found: 513.14854. IR (ATR): 3078, 3004, 2934, 2839, $1593,1509,1341,1245,1159,1089,1029,848,814,782,702,689,657,565,528 \mathrm{~cm}^{-1}$.

\section{3-(4-Chlorophenyl)-4-(4-methoxyphenyl)-2H-chromene (22c).}

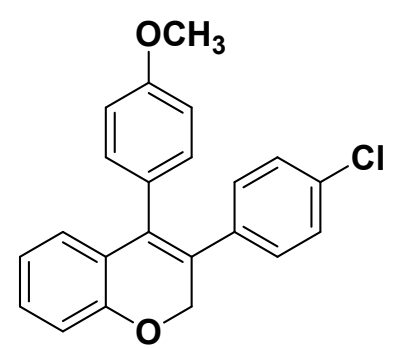

Prepared according to the general procedure using 1-methoxy-4-(3phenoxyprop-1-yn-1-yl)benzene (24.1 mg, $0.1 \mathrm{mmol}), \mathrm{Ph}_{3} \mathrm{PAuCl}(5$ $\mathrm{mg}, 0.01 \mathrm{mmol})$, 4-chlorobenzendiazonium tetrafluoroborate (34.3 $\mathrm{mg}, 1.5$ equiv, $0.15 \mathrm{mmol})$, ascorbic acid $(10 \mu \mathrm{L}, 0.005 \mathrm{mmol})$, DTBPy $(22.7 \mu \mathrm{L}, 0.1 \mathrm{mmol})$ and acetonitrile $(1.5 \mathrm{~mL})$. Purification by column chromatography (silica gel, hexane/EtOAc 98:2) gave the product ( $24 \mathrm{mg}, 68 \%$ ) as a pale yellow solid; m.p. $134-135^{\circ} \mathrm{C} .{ }^{1} \mathbf{H}$ NMR $\left(\mathbf{5 0 0} \mathbf{~ M H z}, \mathbf{C D C l}_{\mathbf{3}}\right) \delta 7.19-7.15(\mathrm{~m}, 1 \mathrm{H})$, $7.14-7.11(\mathrm{~m}, 2 \mathrm{H}), 7.04-6.99(\mathrm{~m}, 2 \mathrm{H}), 6.95-6.90(\mathrm{~m}, 3 \mathrm{H}), 6.87-6.84(\mathrm{~m}, 2 \mathrm{H}), 6.84-$ 6.81 (m, 2H), 5.05 (s, 2H), 3.81 (s, 3H). ${ }^{13} \mathbf{C}$ NMR (125 MHz, $\left.\mathbf{C D C l}_{3}\right) \delta$ 158.96, 153.97, 136.94, 133.27, 132.67, 131.86, 129.61, 129.30, 128.79, 128.36, 128.01, 126.74, 125.54, 121.56, 115.93, 113.95, 69.43, 55.32. HRMS-DART calculated for $\mathrm{C}_{22} \mathrm{H}_{18} \mathrm{ClO}_{2}[\mathrm{M}+\mathrm{H}]^{+}$: 349.09953; found: 349.09879. IR (ATR): 2959, 2926, 2836, 1603, 1508, 1460, 1211, 751 $\mathrm{cm}^{1}$.

\section{3-(4-Bromophenyl)-4-(4-methoxyphenyl)-2H-chromene (23c).}

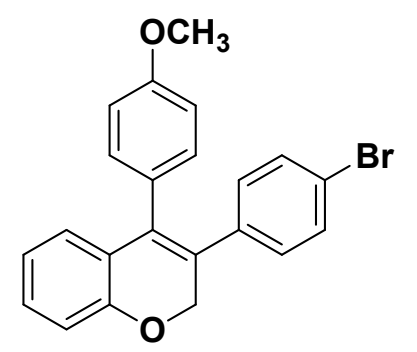

Prepared according to the general procedure using 1-methoxy-4-(3phenoxyprop-1-yn-1-yl)benzene (24.1 mg, $0.1 \mathrm{mmol}$ ), $\mathrm{Ph}_{3} \mathrm{PAuCl}$ (5 mg, $0.01 \mathrm{mmol}$ ), 4-bromobenzendiazonium tetrafluoroborate 
(41.1 mg, 1.5 equiv, $0.15 \mathrm{mmol}$ ), ascorbic acid (10 $\mu \mathrm{L}, 0.005 \mathrm{mmol})$, DTBPy $(22.7 \mu \mathrm{L}, 0.1$ $\mathrm{mmol}$ ) and acetonitrile $(1.5 \mathrm{~mL})$. Purification by column chromatography (silica gel, hexane/EtOAc 95:5) gave the product (21.9 mg, 55\%) as a pale yellow solid. m.p. 144-145 ${ }^{\circ} \mathrm{C} .{ }^{1} \mathbf{H}$ NMR (500 MHz, $\left.\mathbf{C D C l}_{3}\right) \delta 7.30-7.27(\mathrm{~m}, 2 \mathrm{H}), 7.17$ (ddd, $\left.J=8.1,6.1,2.9 \mathrm{~Hz}, 1 \mathrm{H}\right)$, $7.04-6.99(\mathrm{~m}, 2 \mathrm{H}), 6.95-6.91(\mathrm{~m}, 1 \mathrm{H}), 6.88-6.84(\mathrm{~m}, 4 \mathrm{H}), 6.84-6.80(\mathrm{~m}, 2 \mathrm{H}), 5.05(\mathrm{~s}$, 2H), 3.81 (s, 3H). ${ }^{13} \mathbf{C}$ NMR (125 MHz, $\mathbf{C D C l}_{\mathbf{3}}$ ) $\delta$ 158.97, 153.97, 137.41, 133.31, 131.85, $131.30,129.92,129.32,128.77,128.00,126.75,125.55,121.57,120.88,115.94,113.97$, 69.36, 55.32. HRMS-DART calculated for $\mathrm{C}_{22} \mathrm{H}_{18} \mathrm{BrO}_{2}[\mathrm{M}+\mathrm{H}]^{+}$: 393.04902; found: 393.04857. IR (ATR): 2956, 2924, 2850, 1598, 1507, 1482, 1446, 1240, 1211, 801, 766 $\mathrm{cm}^{-1}$.

\section{3-(4-Fluorophenyl)-4-(4-methoxyphenyl)-2H-chromene (24c).}<smiles>COc1ccc(C2=C(c3ccc(F)cc3)COc3ccccc32)cc1</smiles>

Prepared according to the general procedure using 1-methoxy-4-(3phenoxyprop-1-yn-1-yl)benzene (48.2 mg, $0.2 \mathrm{mmol}$ ), $\mathrm{Ph}_{3} \mathrm{PAuCl}$ (10 mg, $0.02 \mathrm{mmol})$, 4-fluorobenzendiazonium tetrafluoroborate (63.6 mg, 1.5 equiv, $0.3 \mathrm{mmol})$, ascorbic acid $(20 \mu \mathrm{L}, 0.005 \mathrm{mmol})$, DTBPy $(45.4 \mu \mathrm{L}, 0.1 \mathrm{mmol})$, acetonitrile $(3 \mathrm{~mL})$ and heated at 50 ${ }^{\circ} \mathrm{C}$. Purification by column chromatography (silica gel, hexane/EtOAc 98:2) gave the product (29.5 mg, 44\%) as a pale yellow solid; m.p. 154-156 ${ }^{\circ} \mathrm{C} .{ }^{1} \mathbf{H}$ NMR (400 MHz, $\left.\mathbf{C D C l}_{3}\right) \delta 7.16(\mathrm{ddd}, J=8.1,6.1,2.9 \mathrm{~Hz}, 1 \mathrm{H}), 7.04-7.00$ (m, 2H), $6.99-6.95(\mathrm{~m}, 2 \mathrm{H}), 6.95-6.91(\mathrm{~m}, 1 \mathrm{H}), 6.88-6.84(\mathrm{~m}, 3 \mathrm{H}), 6.84-6.80(\mathrm{~m}, 3 \mathrm{H}), 5.06(\mathrm{~s}$, 2H), $3.81(\mathrm{~s}, 3 \mathrm{H}) .{ }^{13} \mathbf{C}$ NMR (100 MHz, $\left.\mathbf{C D C l}_{3}\right) \delta 161.56\left(\mathrm{~d}, J_{C-F}=246.8 \mathrm{~Hz}\right), 158.90$, $153.93,134.50\left(\mathrm{~d}, J_{C-F}=3.2 \mathrm{~Hz}\right), 132.84,131.90,130.00\left(\mathrm{~d}, J_{C-F}=7.9 \mathrm{~Hz}\right), 129.15,128.95$, $128.37,126.64,125.60,121.52,115.90,115.14\left(\mathrm{~d}, J_{C-F}=21.4 \mathrm{~Hz}\right), 113.92,69.58,55.32 .{ }^{19} \mathbf{F}$ NMR (375 MHz, CDCl $) \delta-114.50$. HRMS-DART calculated for $\mathrm{C}_{22} \mathrm{H}_{18} \mathrm{FO}_{2}[\mathrm{M}+\mathrm{H}]^{+}$: 333.12908; found: 333.12854. IR (ATR): 2956, 2837, 1740, 1600, 1506, 1452, 1245, 811, $729 \mathrm{~cm}^{-1}$.

Ethyl 4-(4-(4-Methoxyphenyl)-2H-chromen-3-yl)benzoate (25c).

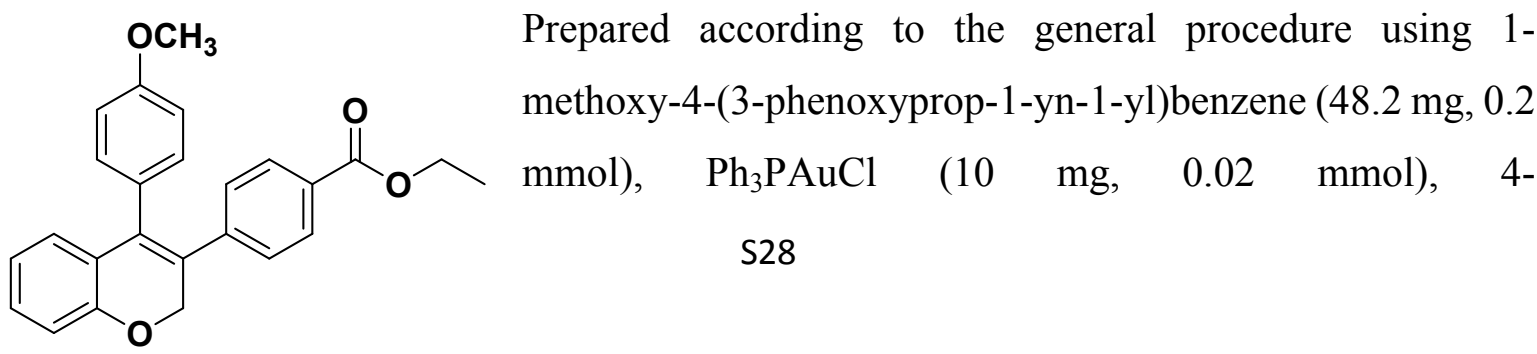


(ethoxycarbonyl)benzenediazonium tetrafluoroborate ( $80 \mathrm{mg}, 1.5$ equiv, $0.3 \mathrm{mmol}$ ), ascorbic acid $(20 \mu \mathrm{L}, 0.01 \mathrm{mmol})$, DTBPy $(45.4 \mu \mathrm{L}, 0.2 \mathrm{mmol})$ and acetonitrile $(3 \mathrm{~mL})$. Purification by column chromatography ( silica gel, hexane/Et $\left.{ }_{2} \mathrm{O} 95: 5\right)$ gave the product $(38.1 \mathrm{mg}, 49 \%$ ) as a pale orange solid; m.p. $121-123{ }^{\circ} \mathrm{C} .{ }^{1} \mathbf{H}$ NMR (400 $\left.\mathbf{M H z}, \mathbf{C D C l}_{3}\right) \delta 7.87-7.80(\mathrm{~m}, 2 \mathrm{H})$, 7.18 (ddd, $\mathrm{J}=8.1,6.5,2.5 \mathrm{~Hz}, 1 \mathrm{H}), 7.07-7.03(\mathrm{~m}, 2 \mathrm{H}), 7.03-6.99(\mathrm{~m}, 2 \mathrm{H}), 6.97-6.92$ (m, 1H), $6.90-6.84(\mathrm{~m}, 2 \mathrm{H}), 6.84-6.78(\mathrm{~m}, 2 \mathrm{H}), 5.09(\mathrm{~s}, 2 \mathrm{H}), 4.33$ (q, J = 7.1 Hz, 2H), $3.80(\mathrm{~s}, 3 \mathrm{H}), 1.36(\mathrm{t}, \mathrm{J}=7.1 \mathrm{~Hz}, 3 \mathrm{H}) .{ }^{13} \mathbf{C} \mathbf{N M R}\left(\mathbf{1 0 0} \mathbf{M H z}, \mathbf{C D C l}_{3}\right) \delta 166.44,159.08,154.16$, $143.21,134.14,131.87,129.53,129.38,129.25,128.76,128.71,128.22,126.92,125.50$, 121.60, 116.00, 113.98, 69.37, 61.07, 55.31, 14.46. HRMS-DART calculated for $\mathrm{C}_{25} \mathrm{H}_{23} \mathrm{O}_{4}$ $[\mathrm{M}+\mathrm{H}]^{+}:$387.15963; found: 387.15906. IR (ATR): 2958, 2868, 1712. 1604, 1510, 1463, $1271,1244 \mathrm{~cm}^{-1}$.

(4-(4-(4-Methoxyphenyl)-2H-chromen-3-yl)phenyl)(phenyl)methanone (26c).<smiles>COc1ccc(C2=C(c3ccc(C(=O)c4ccccc4)cc3)COc3ccccc32)cc1</smiles>
Prepared according to the general procedure using 1methoxy-4-(3-phenoxyprop-1-yn-1-yl)benzene (96.3 mg, 0.1 $\mathrm{mmol}), \quad \mathrm{Ph}_{3} \mathrm{PAuCl} \quad(20 \quad \mathrm{mg}, \quad 0.01 \quad \mathrm{mmol})$, 4benzoylbenzendiazonium tetrafluoroborate $(179.5 \mathrm{mg}, 1.5$ equiv, $0.15 \mathrm{mmol})$, ascorbic acid $(40 \mu \mathrm{L}, 0.005 \mathrm{mmol})$, DTBPy $(90.8 \mu \mathrm{L}, 0.1 \mathrm{mmol})$ and acetonitrile $(6 \mathrm{~mL})$. Purification by column chromatography (silica gel, hexane/EtOAc 9:1) gave the product (77 mg, 45\%) as a pale yellow solid; m.p. 142-144 ${ }^{\circ}$ C. ${ }^{1} \mathbf{H}$ NMR (300 MHz, $\left.\mathbf{C D C l}_{3}\right) \delta 7.77-7.71(\mathrm{~m}, 2 \mathrm{H}), 7.67-7.60(\mathrm{~m}, 2 \mathrm{H}), 7.56$ $(\mathrm{dt}, J=6.6,1.7 \mathrm{~Hz}, 1 \mathrm{H}), 7.50-7.42(\mathrm{~m}, 2 \mathrm{H}), 7.19(\mathrm{ddd}, J=8.1,6.2,2.8 \mathrm{~Hz}, 1 \mathrm{H}), 7.13-$ $7.07(\mathrm{~m}, 2 \mathrm{H}), 7.07-7.00(\mathrm{~m}, 2 \mathrm{H}), 6.95$ (d, J=7.9 Hz, 1H), $6.91-6.85(\mathrm{~m}, 2 \mathrm{H}), 6.85-6.78$ (m, 2H), 5.12 (s, 2H), 3.80 (s, 3H). ${ }^{13} \mathbf{C}$ NMR (75 MHz, CDCl $) \delta$ 196.30, 159.14, 154.18, $142.98,137.74,135.72,134.36,132.48,131.90,130.09,130.06,129.60,128.70,128.40$, $128.18,128.06,126.98,125.54,121.65,116.02,114.03,69.40,55.33$. HRMS-DART calculated for $\mathrm{C}_{29} \mathrm{H}_{23} \mathrm{O}_{3}[\mathrm{M}+\mathrm{H}]^{+}:$419.16472; found: 419.16352. IR (ATR): 2959, 2837, $1651,1595,1508,1245,1218,1171 \mathrm{~cm}^{-1}$.

\section{4-(4-(4-Methoxyphenyl)-2H-chromen-3-yl)benzonitrile (27c).}




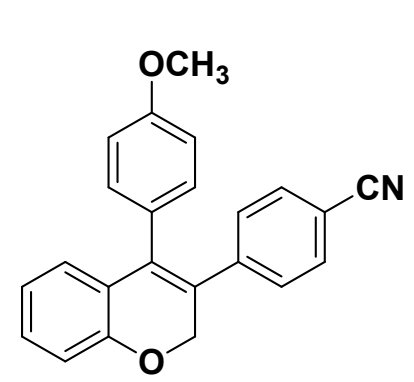

Prepared according to the general procedure using 1-methoxy-4(3-phenoxyprop-1-yn-1-yl)benzene (96.3 $\mathrm{mg}, \quad 0.4 \mathrm{mmol}$ ), $\mathrm{Ph}_{3} \mathrm{PAuCl} \quad(20 \mathrm{mg}, \quad 0.04 \mathrm{mmol})$, 4-cyanobenzendiazonium tetrafluoroborate ( $131.5 \mathrm{mg}, 1.5$ equiv, $0.6 \mathrm{mmol})$, ascorbic acid (40 $\mu \mathrm{L}, 0.02 \mathrm{mmol})$, DTBPy $(90.8 \mu \mathrm{L}, 0.4 \mathrm{mmol})$ and acetonitrile

(6 mL). Purification by column chromatography (silica gel, hexane/EtOAc 9:1) gave the product (101.3 mg, $74 \%$ ) as a white solid; m.p. $129-130{ }^{\circ} \mathrm{C} .{ }^{1} \mathbf{H}$ NMR (500 MHz, $\left.\mathbf{C D C l}_{3}\right) \delta 7.46-7.42(\mathrm{~m}, 2 \mathrm{H}), 7.20$ (ddd, $\left.J=8.1,6.0,3.0 \mathrm{~Hz}, 1 \mathrm{H}\right), 7.10-$ $7.04(\mathrm{~m}, 2 \mathrm{H}), 7.02-6.97(\mathrm{~m}, 2 \mathrm{H}), 6.95(\mathrm{~d}, J=7.8 \mathrm{~Hz}, 1 \mathrm{H}), 6.89-6.86(\mathrm{~m}, 2 \mathrm{H}), 6.85-6.81$ (m, 2H), 5.06 (s, 2H), 3.81 (s, 3H). ${ }^{13}$ C NMR (125 MHz, CDCl $)$ ) $\delta$ 159.16, 154.06, 143.23, 135.07, 131.82, 131.69, 129.80, 128.79, 128.10, 126.97, 126.92, 125.09, 121.61, 118.79, 115.96, 113.98, 110.21, 68.93, 55.22. HRMS-DART calculated for $\mathrm{C}_{23} \mathrm{H}_{18} \mathrm{NO}_{2}[\mathrm{M}+\mathrm{H}]^{+}$: 340.13375; found: 340.13308. IR (ATR): 2901, 2833, 2222, 1595, 1510, 1481, 1249, 1215 $\mathrm{cm}^{-1}$.

\section{4-(4-Methoxyphenyl)-3-(3-nitrophenyl)-2H-chromene (28c).}

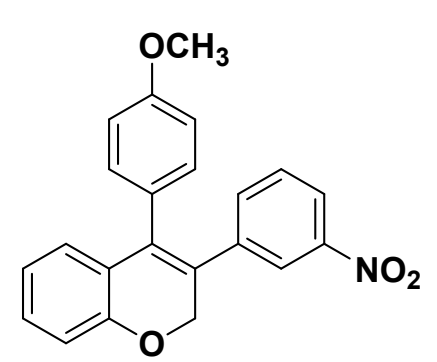

Prepared according to the general procedure using 1-methoxy-4(3-phenoxyprop-1-yn-1-yl)benzene (24.1 $\mathrm{mg}, \quad 0.1 \mathrm{mmol})$, $\mathrm{Ph}_{3} \mathrm{PAuCl} \quad(5 \mathrm{mg}, \quad 0.01 \mathrm{mmol}), \quad 3$-nitrobenzendiazonium tetrafluoroborate $(35.9 \mathrm{mg}, 1.5$ equiv, $0.15 \mathrm{mmol})$, ascorbic acid

(10 $\mu \mathrm{L}, 0.005 \mathrm{mmol})$, DTBPy $(22.7 \mu \mathrm{L}, 0.1 \mathrm{mmol})$ and acetonitrile $(1.5 \mathrm{~mL})$. Purification by column chromatography (silica gel, hexane/EtOAc 95:5) gave the product (19.5 mg, 54\%) as an orange solid; m.p. $146-147^{\circ} \mathrm{C} .{ }^{1} \mathbf{H}$ NMR (500 MHz, $\left.\mathbf{C D C l}_{3}\right) \delta 7.92(\mathrm{ddd}, J=7.9,2.2,1.5 \mathrm{~Hz}, 1 \mathrm{H}), 7.83(\mathrm{t}, J=1.8 \mathrm{~Hz}, 1 \mathrm{H}), 7.24(\mathrm{~d}, J=7.8$ Hz, 1H), $7.22-7.20$ (m, 1H), 7.14 (ddd, $J=8.1,5.6,3.4 \mathrm{~Hz}, 1 \mathrm{H}), 6.99-6.94(\mathrm{~m}, 2 \mathrm{H}), 6.90$ $(\mathrm{d}, J=7.9 \mathrm{~Hz}, 1 \mathrm{H}), 6.83-6.80(\mathrm{~m}, 2 \mathrm{H}), 6.79-6.75(\mathrm{~m}, 2 \mathrm{H}), 5.04(\mathrm{~s}, 2 \mathrm{H}), 3.74(\mathrm{~s}, 3 \mathrm{H}) .{ }^{13} \mathrm{C}$ NMR (125 MHz, $\left.\mathbf{C D C l}_{3}\right) \delta 159.27,154.09,148.11,140.17,135.17,134.58,131.80,129.88$, 129.03, 128.08, 127.05, 126.55, 125.13, 123.03, 121.86, 121.73, 116.08, 114.19, 69.07, 55.37. HRMS-DART calculated for $\mathrm{C}_{22} \mathrm{H}_{18} \mathrm{NO}_{4}[\mathrm{M}+\mathrm{H}]^{+}: 360.12358$; found: 360.12233 . IR (ATR): 2958, 2931, 2836, 1604, 1509, 1454, 1288, $1244 \mathrm{~cm}^{-1}$.

\section{3-(4-(4-Methoxyphenyl)-2H-chromen-3-yl)benzonitrile (29c).}




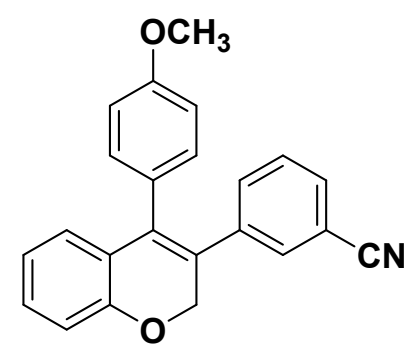

Prepared according to the general procedure using 1-methoxy-4(3-phenoxyprop-1-yn-1-yl)benzene (24.1 $\mathrm{mg}, \quad 0.1 \mathrm{mmol})$, $\mathrm{Ph}_{3} \mathrm{PAuCl} \quad(5 \mathrm{mg}, \quad 0.01 \mathrm{mmol}$ ), 3-cyanobenzendiazonium tetrafluoroborate ( $32.9 \mathrm{mg}, 1.5$ equiv, $0.15 \mathrm{mmol}$ ), ascorbic acid (10 $\mu \mathrm{L}, 0.005 \mathrm{mmol})$, DTBPy $(22.7 \mu \mathrm{L}, 0.1 \mathrm{mmol})$ and acetonitrile $(1.5 \mathrm{~mL})$. Purification by column chromatography (silica gel, hexane/EtOAc 9:1) gave the product (20.8 $\mathrm{mg}, 60 \%$ ) as a pale yellow solid; m.p. 129-130 ${ }^{\circ} \mathrm{C} .{ }^{1} \mathbf{H}$ NMR (300 MHz, $\left.\mathbf{C D C l}_{3}\right) \delta 7.41(\mathrm{dt}, J=7.4,1.5 \mathrm{~Hz}, 1 \mathrm{H}), 7.31-7.15(\mathrm{~m}, 4 \mathrm{H}), 7.03$ $-6.97(\mathrm{~m}, 2 \mathrm{H}), 6.95(\mathrm{~d}, J=8.1 \mathrm{~Hz}, 1 \mathrm{H}), 6.86(\mathrm{~d}, J=4.1 \mathrm{~Hz}, 2 \mathrm{H}), 6.85-6.79(\mathrm{~m}, 2 \mathrm{H}), 5.05$ (s, 2H), 3.81 (s, 3H). ${ }^{13} \mathbf{C}$ NMR (75 MHz, $\mathbf{C D C l}_{3}$ ) $\delta$ 159.25, 154.08, 139.89, 134.82, 132.81, 131.81, 131.73, 130.48, 129.81, 129.01, 128.10, 127.00, 126.72, 125.16, 121.71, 118.73, 116.06, 114.14, 112.43, 69.09, 55.36. HRMS-DART calculated for $\mathrm{C}_{23} \mathrm{H}_{18} \mathrm{NO}_{2}[\mathrm{M}+\mathrm{H}]^{+}$: 340.13375; found: 340.13453. IR (ATR): 2922, 2854, 2227, 1607, 1510, 1446, 1288, 1248 , $1174 \mathrm{~cm}^{-1}$.

\section{3-(3-Chlorophenyl)-4-(4-methoxyphenyl)-2H-chromene (30c).}

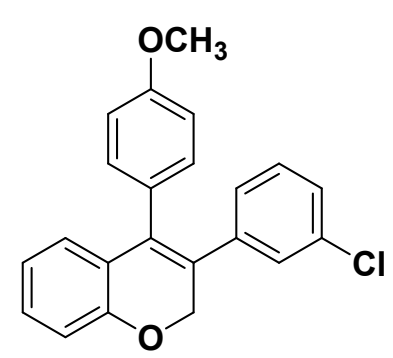

Prepared according to the general procedure using 1-methoxy-4-(3phenoxyprop-1-yn-1-yl)benzene (48.2 mg, $0.2 \mathrm{mmol}$ ), $\mathrm{Ph}_{3} \mathrm{PAuCl}$ (10 mg, $0.02 \mathrm{mmol}$ ), 3-chlorobenzendiazonium tetrafluoroborate (91.5 mg, 1.5 equiv, $0.3 \mathrm{mmol})$, ascorbic acid ( $20 \mu \mathrm{L}, 0.01 \mathrm{mmol})$, DTBPy $(45.4 \mu \mathrm{L}, 0.2 \mathrm{mmol})$, acetonitrile $(3 \mathrm{~mL})$ and heated at 50

${ }^{\circ} \mathrm{C}$. Purification by column chromatography (silica gel, hexane/Et $2 \mathrm{O}$ 95:5) gave the product (40.4 mg, 57\%) as a pale yellow solid; m.p. $101-103{ }^{\circ} \mathrm{C} .{ }^{1} \mathbf{H}$ NMR (300 MHz, $\left.\mathbf{C D C l}_{3}\right) \delta 7.21-7.12(\mathrm{~m}, 1 \mathrm{H}), 7.12-6.98(\mathrm{~m}, 6 \mathrm{H}), 6.93(\mathrm{~d}, J=7.9 \mathrm{~Hz}, 1 \mathrm{H}), 6.90$ - 6.77 (m, 5H), 5.05 (s, 2H), 3.81 (s, 3H). ${ }^{13} \mathbf{C}$ NMR (75 MHz, CDCl $) \delta$ 159.06, 154.06, $140.35,134.03$, 133.75, 131.85, 129.43, 129.37, 128.64, 128.17, 127.90, 127.12, 126.86, 126.75，125.46，121.58，115.97，113.97，69.36，55.36. HRMS-DART calculated for $\mathrm{C}_{22} \mathrm{H}_{18} \mathrm{ClO}_{2}[\mathrm{M}+\mathrm{H}]^{+}$: 349.09953; found: 349.09804. IR (ATR): 3065, 3007, 2928, 2838, $1741,1657,1606,1572,1510,1482,1448,1284,1245,1174,1113,1082,1027,932,871$, $838,790,762,695,601,565,458 \mathrm{~cm}^{-1}$.

\section{4-(4-Methoxyphenyl)-3-(2-nitrophenyl)-2H-chromene (31c).}




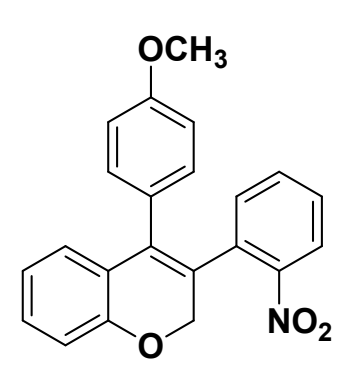

Prepared according to the general procedure using 1-methoxy-4-(3phenoxyprop-1-yn-1-yl)benzene (48.2 mg, $0.2 \mathrm{mmol}), \mathrm{Ph}_{3} \mathrm{PAuCl}(10$ $\mathrm{mg}, 0.02 \mathrm{mmol}), 2$-nitrobenzenediazonium tetrafluoroborate $(71.8 \mathrm{mg}$, 1.5 equiv, $0.3 \mathrm{mmol})$, ascorbic acid $(20 \mu \mathrm{L}, 0.01 \mathrm{mmol})$, DTBPy (45.4 $\mu \mathrm{L}, 0.2 \mathrm{mmol})$, acetonitrile $(3 \mathrm{~mL})$ and heated at $50^{\circ} \mathrm{C}$. Purification by column chromatography (silica gel, hexane/Et ${ }_{2} \mathrm{O} 9: 1$ ) gave the product (30 mg, 45\%) as an orange oil. ${ }^{1} \mathbf{H}$ NMR (300 MHz, $\left.\mathbf{C D C l}_{3}\right) \delta 7.94(\mathrm{dd}, J=8.2,1.3 \mathrm{~Hz}, 1 \mathrm{H})$, 7.41 (td, $J=7.5,1.4 \mathrm{~Hz}, 1 \mathrm{H}), 7.31$ (ddd, $J=8.1,7.4,1.6 \mathrm{~Hz}, 1 \mathrm{H}), 7.23-7.13(\mathrm{~m}, 2 \mathrm{H}), 6.98$ $-6.91(\mathrm{~m}, 3 \mathrm{H}), 6.90-6.84(\mathrm{~m}, 2 \mathrm{H}), 6.76-6.68(\mathrm{~m}, 2 \mathrm{H}), 5.09$ (bs, 1H), 4.79 (bs, 1H), 3.74 (s, 3H). ${ }^{13} \mathbf{C}$ NMR (75 MHz, $\left.\mathbf{C D C l}_{3}\right) \delta 158.97,154.36,148.60,134.62,133.77,133.45$, 132.70, 131.26, 129.60, 128.36, 128.34, 127.60, 126.86, 124.99, 124.64, 121.57, 116.07, 113.75, 68.74, 55.25. HRMS-DART calculated for $\mathrm{C}_{22} \mathrm{H}_{18} \mathrm{NO}_{4}[\mathrm{M}+\mathrm{H}]^{+}: 360.12358$; found: 360.12437. IR (ATR): 2956, 2933, 2910, 2860, 2837, 1607, 1571, 1522, 1510, 1483, 1453, $1343,1288,1243,1212,1174,1109,1059,1031,1002,908,828,78,729,704,596,564$, $541 \mathrm{~cm}^{-1}$.

\section{Solid-state studies for 4-(4-Methoxyphenyl)-3-(4-nitrophenyl)-2H- chromene 2c.}

\section{Experimental details}

Single-crystal X-ray diffraction: Data collection were performed at $150 \mathrm{~K}$ on a BrukerAPEX-II CCD diffractometer with Mo K $\alpha$-radiation $(\lambda=0.71073 \AA$ ). Data collection and cell parameter calculations were performed using APEX II software. Structure solution and 
refinement were accomplished using SHELX crystallography packages. ${ }^{[18]}$ All non-hydrogen atoms were refined anisotropically and their positions were calculated using HFIX command.

Powder X-ray diffraction: Analyses were carried out at room temperature in the range of $2 \theta=5-45^{\circ}$, step size of $0.03^{\circ}$, voltage of $40 \mathrm{kV}$ and step time of $40.005 \mathrm{~s}$ on a Bruker D2 Phaser diffractometer using $\mathrm{Cu}-\mathrm{K}_{\alpha 1}=1.5406 \AA$ radiation.

${ }^{13} \mathrm{C}$ CP-MAS NMR: The spectra were recorded at $25{ }^{\circ} \mathrm{C}$ with natural abundance ${ }^{13} \mathrm{C}$ powdered samples spun at $15 \mathrm{kHz}$ on a Bruker Ascend 500 solid-state spectrometer utilizing $3.2 \mathrm{~mm} \mathrm{HXY} \mathrm{CP} / \mathrm{MAS}$ probe at $125.78 \mathrm{MHz}$ with spinning rate of $10 \mathrm{kHz}$. Measures were acquired using a contact time of $3 \mathrm{~ms}$, a delay of $10 \mathrm{~s}$ and the chemical shifts were referenced using the peaks of adamantane at 37.7 and $28.7 \mathrm{ppm}$.

Thermal Analysis: Differential scanning calorimetry (DSC) and Thermogravimetric Analysis (TGA) experiments were determined in a simultaneous TGA-DSC Netzsch STA 449 F3 Jupiter instrument. Samples were taken on an aluminum pans and heated from room temperature to $200^{\circ} \mathrm{C}$ at a rate of $10^{\circ} \mathrm{C} / \mathrm{min}$ under nitrogen atmosphere.

Table S1. Crystallographic and refinement data of polymorphic forms $2 \mathbf{c}(\mathbf{I})$ and $2 \mathrm{c}(\mathrm{II})$

\begin{tabular}{c|cc}
\hline Compound & $\mathbf{2 c ( I )}$ & $\mathbf{2 c ( I I )}$ \\
\hline formula & $\mathrm{C}_{22} \mathrm{H}_{17} \mathrm{NO}_{4}$ & $\mathrm{C}_{22} \mathrm{H}_{17} \mathrm{NO}_{4}$ \\
formula weight & 359.37 & 359.37 \\
temperature/K & 150 & 150 \\
crystal system & triclinic & monoclinic \\
space group & $P-1$ & $P 2_{1}$ \\
\hline
\end{tabular}




\begin{tabular}{|c|c|c|}
\hline$a / \AA$ & $6.2331(9)$ & $5.9302(3)$ \\
\hline$b / \AA$ & $10.6409(16)$ & $21.3945(10)$ \\
\hline$c / \AA ̊$ & $14.154(2)$ & $13.9168(6)$ \\
\hline$\alpha /^{o}$ & $100.755(4)$ & 90 \\
\hline$\beta /^{\circ}$ & $94.852(3)$ & $95.871(1)$ \\
\hline$\gamma^{o}$ & $105.369(3)$ & 90 \\
\hline volume $/ \AA^{3}$ & $880.4(2)$ & $1756.41(14)$ \\
\hline Z & 2 & 4 \\
\hline$\rho$ calc $\mathrm{g} / \mathrm{cm}^{3}$ & 1.356 & 1.359 \\
\hline$\mu / \mathrm{mm}^{-1}$ & 0.094 & 0.094 \\
\hline$F(000)$ & 376.0 & 752.0 \\
\hline crystal size & $0.024 \times 0.098 \times 0.407$ & $0.056 \times 0.169 \times 0.328$ \\
\hline radiation $/ \AA$ & MoKa 0.71073 & MoKa 0.71073 \\
\hline $2 \theta$ range & 2.24 to 27.2 & 2.41 to 30.10 \\
\hline index ranges & $\begin{array}{c}-8 \leq \mathrm{h} \leq 8 \\
-13 \leq \mathrm{k} \leq 13\end{array}$ & $\begin{array}{c}-8 \leq \mathrm{h} \leq 8 \\
-29 \leq \mathrm{k} \leq 29\end{array}$ \\
\hline reflns collected & $\begin{array}{c}-18 \leq 1 \leq 18 \\
13375\end{array}$ & $\begin{array}{c}-19 \leq 1 \leq 19 \\
44739\end{array}$ \\
\hline Independent reflns & 4023 & 9844 \\
\hline reflns observed & 1531 & 6591 \\
\hline GOF on $\mathrm{F}^{2}$ & 0.983 & 1.023 \\
\hline $\begin{array}{l}\text { Final } \mathrm{R} \text { indexes } \\
\qquad[I \geq 2(I)]\end{array}$ & $\begin{array}{c}\mathrm{R}=0.0819 \\
\mathrm{wR}_{2}=0.2115\end{array}$ & $\begin{array}{c}\mathrm{R}=0.0546 \\
\mathrm{wR}_{2}=0.1208\end{array}$ \\
\hline CCDC No. & 2046490 & 2046491 \\
\hline
\end{tabular}




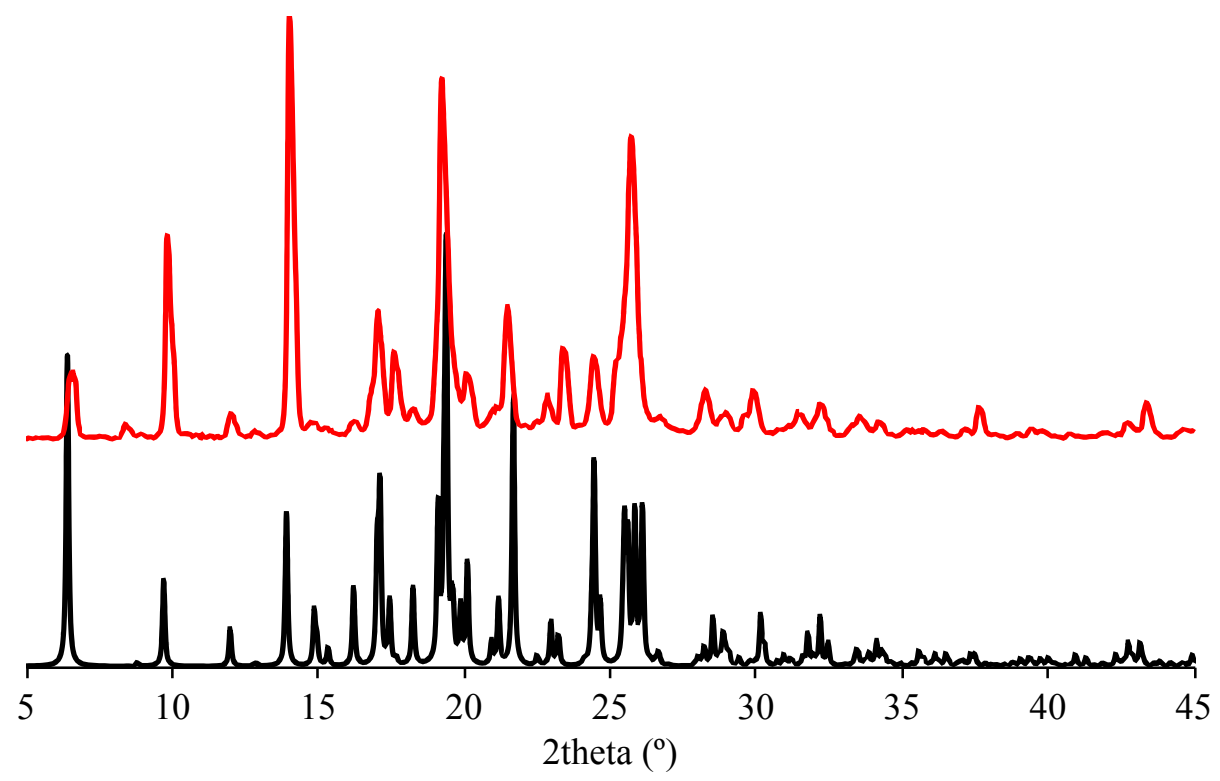

Figure S1. Comparison of powder X-ray diffraction patterns for solid form $\mathbf{2 c ( I ) . ~ ( T o p ) ~}$ Experimental. (Bottom) Calculated from crystalline structure.

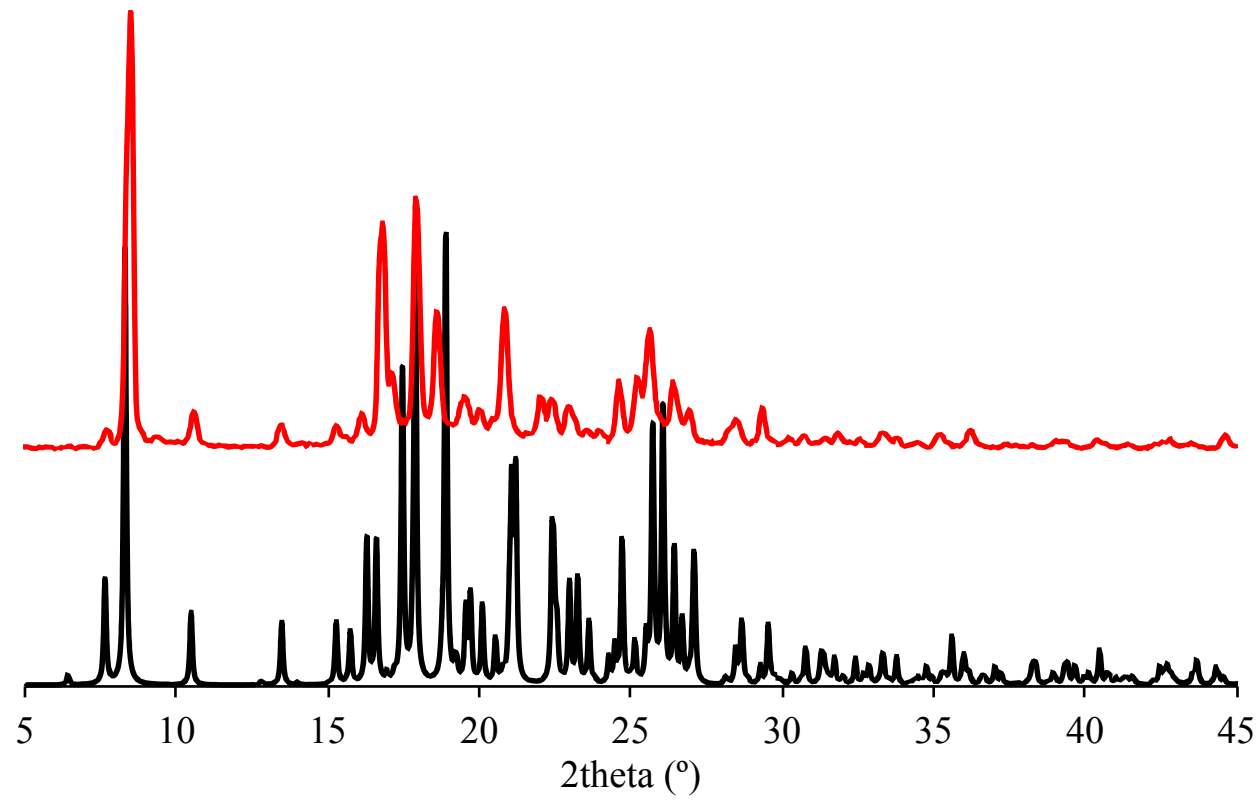

Figure S2. Comparison of powder X-ray diffraction patterns for solid form 2c(II). (Top) Experimental. (Bottom) Calculated from crystalline structure. 


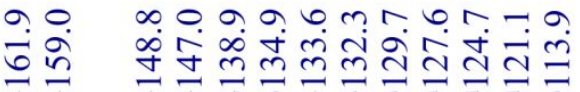

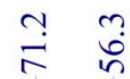

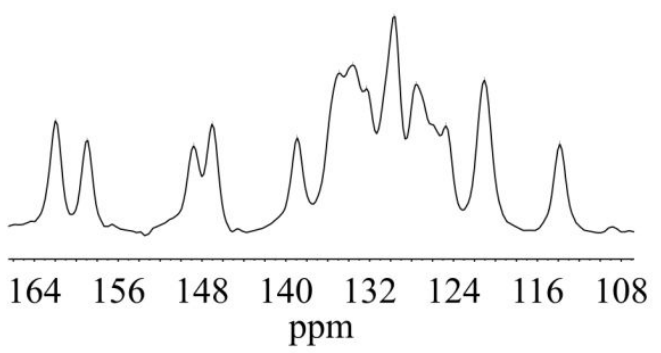<smiles>COc1ccc(C2=C(c3ccc([N+](=O)[O-])cc3)COc3ccccc32)cc1</smiles>

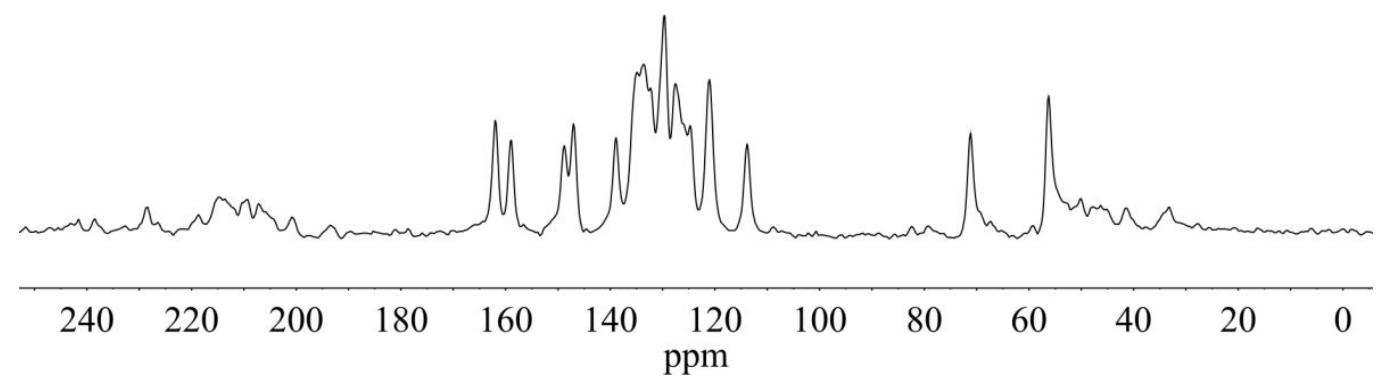

Figure S3. Solid-state ${ }^{13} \mathrm{C}$ CPMAS NMR spectrum (125 MHz) of polymorphic form $2 \mathbf{c}(\mathrm{I})$ at room temperature.

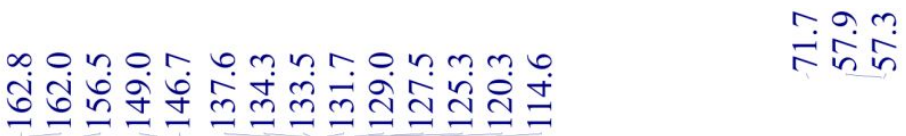
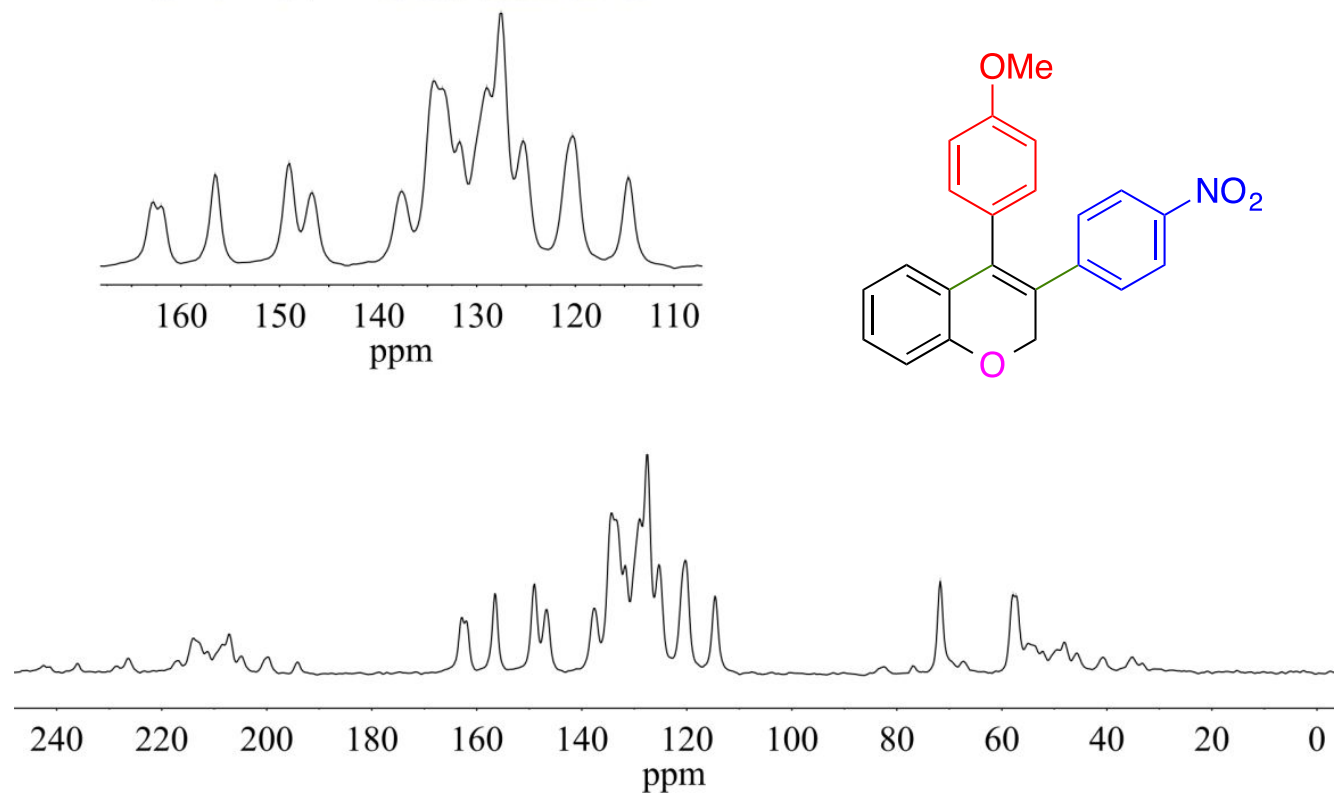

Figure S4. Solid-state ${ }^{13} \mathrm{C}$ CPMAS NMR spectrum $(125 \mathrm{MHz})$ of polymorphic form $\mathbf{2 c ( I I )}$ at room temperature. 


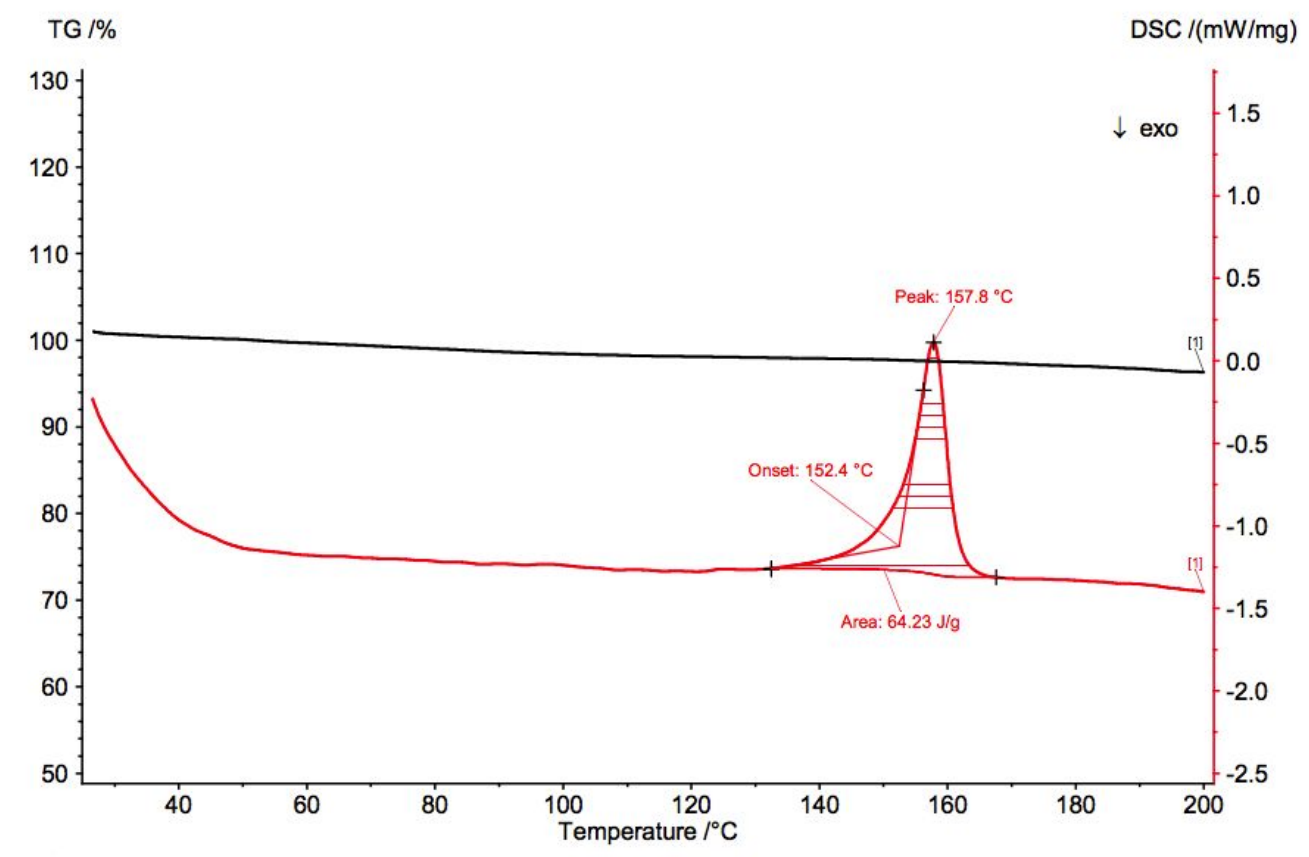

Figure S1. Thermal analyses of solid form $\mathbf{2 c ( I ) . ~ D i f f e r e n t i a l ~ S c a n n i n g ~ C a l o r i m e t r y ~ ( r e d ~ l i n e ) ~ a n d ~}$ thermogravimetric analysis (black line).

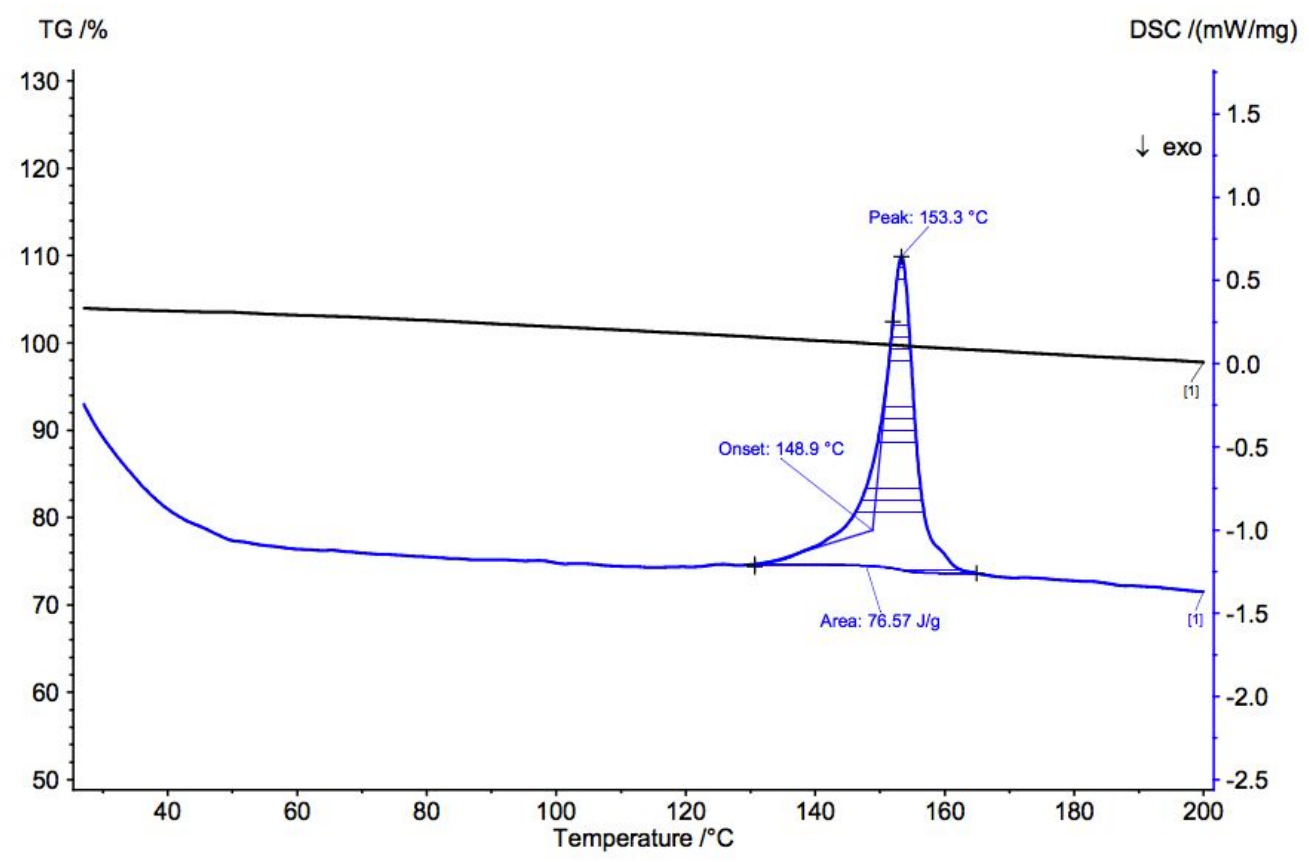

Figure S2. Thermal analyses of solid form 2c(II). Differential Scanning Calorimetry (blue line) and thermogravimetric analysis (black line). 


\section{Computational details.}

All the calculations were perform with the M06 functional, the Def2TZVP basis set and Grimme empirical dispersion method implemented in Gaussian 16. ${ }^{19-22}$ All the structures were fully optimized and a frequency calculation was performed to ensure that the optimized structures were a true minimum or a saddle point of first order. To verify the lack of energetic barrier of the bimolecular steps, a scan calculation was carry out in which the scanned variable was the distance between the two reacting molecules. The energy always decrees with the distances, in these barrierless steps.

\section{References.}

[1] Armarego, W. L. F.; Chai, C. L. L. Purification of Laboratory Chemicals, ELSEVIER SCIENCE \& TECHNOLOGY, 2003.

[2] Qiu, W. W.; Surendra, K.; Yin, L.; Corey, E. J. Selective formation of six-membered oxa-and carbocycles by the In(III)-activated ring closure of acetylenic substrates. Org. Lett. 2011, 13, 5893-5895.

[3] Worlikar, S. A.; Kesharwani, T.; Yao, T.; Larock, R. C. Synthesis of 3,4-disubstituted 2Hbenzopyrans through C-C bond formation via electrophilic cyclization. J. Org. Chem. 2007, $72,1347-1353$.

[4] Nie, X.; Liu, S.; Zong, Y.; Sun, P.; Bao, J. Facile synthesis of substituted alkynes by nanopalladium catlayzed oxidative cross-coupling reaction of arylborinoic acids with terminal alkynes. J. Organomet. Chem. 2011, 696, 1570-1573.

[5] Raju, S.; Kumar, P. R.; Mukkanti, K.; Annamalai, P.; Pal, M. Facile synthesis of substituted thiophenes via $\mathrm{Pd} / \mathrm{C}-$ mediated sonogashira coupling in water. Bioorganic Med. Chem. Lett. 2006, 16, 6185-6189.

[6] Wang, Y. L.; Zhang, W. M.; Dai, J. J.; Feng, Y. S.; Xu, H. J. Cu-catalyzed intramolecular hydroaylation of alkynes. $R S C A d v$. 2014, 4, 61706-61710.

[7] Arcadi, A.; Blesi, F.; Cacchi, S.; Fabrizi, G.; Goggiamani, A.; Marinelli, F. Gold versus silver catalyzed intramolecular hydroarylation reactions of [(3-arylproo-2-ynyl)oxy]benzene derivatives. Org. Biomol. Chem. 2012, 10, 9700-9708.

[8] Fang, J. D.; Yan, X. B.; Zhou, L.; Wang, Y. Z.; Liu, X. Y. Synthesis of 3-organoselenyl-2Hcoumarins from propargylic aryletheres via oxidative radical cyclization. Adv. Synth. Catal. 2019, 361, 1985-1990.

[9] Kabanyane, S. T.; MaGee, D. I. An efficient and general synthesis of alkyl alkynyl sulfoxides. Can. J. Chem. 1992, 70, 2758-2763.

[10] Paul, K.; Jalal, S.; Kundal, S.; Jana, U. Synthesis of fused dibenzofuran derivatives via palladium-catalyzed domino $\mathrm{C}-\mathrm{C}$ bond formation and iron-catalyzed 
cycloisomeriztion/aromatization. J. Org. Chem. 2016, 81, 1164-1174.

[11] Walkinshaw, A. J.; Xu, W.; Suero, M. G.; Gaunt, M. J. Copper-catalyzed carboarylation of alkynes via vinyl cations. J. Am. Chem. Soc. 2013, 135, 12532-12535.

[12] Doyle, M. P.; Bryker, W. J. Alkyl nitrite-metal halide deamination reactions. 6. Direct synthesis of arenediazonium tetrafluoroborate salts from aromatics amines, tert-butyl nitrite and boron trifluoride ethereate in anhydrous media. J. Org. Chem. 1979, 44, 1572-1574.

[13] Reay, A.J.; Hammarback, L. A.; Bray, J. T. W.; Sheridan, T.; Turnbull, D.; Whitwood, A. C.; Fairlamb, I. J. S. Mild and Regioslective $\mathrm{Pd}(\mathrm{OAc})_{2}$-catalyzed C-H arylation of tryptophans by $\mathrm{ArN}_{2} \mathrm{X}$, promoted by tosic acid. ACS Catal. 2017, 7, 5174-5179.

[14] Xing, B.; C. Ni, C.; Hu, J. Hypervalent Iodine(III)-catalyzed Balz-Schiemann fluorination under mild contidions. Angew. Chemie Int. Ed. 2018, 57, 9896-9900.

[15] Salmi, Z.; Lamouri, A.; Decorse, P.; Jouini, M.; Boussadi, A.; Achard, J.; Gicquel, A.; Mahouche-Chergui, S.; Carbonnier, B.; Chehimi, M. M. Grafting poylmer-protien bioconjugate to boron-doped diamond using aryl diazonium coupling agents. Diam. Relat. Mater. 2013, 40, 60-68.

[16] Gosset, C.; Pellegrini, S.; Jooris, R.; Bousquet, T.; Pelinski, L. Visible-light-mediated hydroxycarbonylation of diazonium salts. Adv. Synth. Catal. 2018, 360, 3401-3405.

[17] Sherborne, G. J.; Gevondian, A. G.; Funes-Ardoiz, I.; Dahiya, A.; Fricke, C.; Schoenebeck, F. Modular and selective arylation of arylgermanes $\left(\mathrm{C}-\mathrm{GeEt}_{3}\right)$ over C-Bpin, C-SiR3 and halogens enabled by light-activated gold catalysis. Angew. Chemie Int. Ed. 2020, 59, 1554315548.

[18] Sheldrick, G. M. A Short History of SHELX. Acta Cryst. A., 2008, 112-122.

[19] Zhao, Y.; Truhlar, D. G. The M06 Suite of Density Functionals for Main Group Thermochemistry, Thermochemical Kinetics, Noncovalent Interactions, Excited States, and Transition Elements: Two New Functionals and Systematic Testing of Four M06-Class Functionals and 12 Other Functionals. Theor. Chem. Acc.2007, 120(1-3), 215-241. https://doi.org/10.1007/s00214-007-0310-x.

[20] Weigend, F.; Ahlrichs, R. Balanced Basis Sets of Split Valence, Triple Zeta Valence and Quadruple Zeta Valence Quality for H to Rn: Design and Assessment of Accuracy. Phys. Chem. Chem. Phys.2005, 7 (18), 3297-3305. https://doi.org/10.1039/B508541A. 
[21] Grimme, S.; Antony, J.; Ehrlich, S.; Krieg, H. A Consistent and Accurate Ab Initio Parametrization of Density Functional Dispersion Correction (DFT-D) for the 94 Elements HPu. J. Chem. Phys. 2010, 132 (15), 154104. https://doi.org/10.1063/1.3382344.

[22] Frisch, M. J.; Trucks, G. W.; Schlegel, H. B.; Scuseria, G. E.; Robb, M. A.; Cheeseman, J. R.; Scalmani, G.; Barone, V.; Petersson, G. A.; Nakatsuji, H.; Li, X.; Caricato, M.; Marenich, A. V.; Bloino, J.; Janesko, B. G.; Gomperts, R.; Mennucci, B.; Hratchian, H. P.; Ortiz, J. V.; Izmaylov, A. F.; Sonnenberg, J. L.; Williams-Young, D.; Ding, F.; Lipparini, F.; Egidi, F.; Goings, J.; Peng, B.; Petrone, A.; Henderson, T.; Ranasinghe, D.; Zakrzewski, V. G.; Gao, J.; Rega, N.; Zheng, G.; Liang, W.; Hada, M.; Ehara, M.; Toyota, K.; Fukuda, R.; Hasegawa, J.; Ishida, M.; Nakajima, T.; Honda, Y.; Kitao, O.; Nakai, H.; Vreven, T.; Throssell, K.; Montgomery, Jr., J. A.; Peralta, J. E.; Ogliaro, F.; Bearpark, M. J.; Heyd, J. J.; Brothers, E. N.; Kudin, K. N.; Staroverov, V. N.; Keith, T. A.; Kobayashi, R.; Normand, J.; Raghavachari, K.; Rendell, A. P.; Burant, J. C.; Iyengar, S. S.; Tomasi, J.; Cossi, M.; Millam, J. M.; Klene, M.; Adamo, C.; Cammi, R.; Ochterski, J. W.; Martin, R. L.; Morokuma, K.; Farkas, O.; Foresman, J. B.; Fox, D. J. Gaussian 16 Revision B.01; 2016.

\section{NMR spectra}

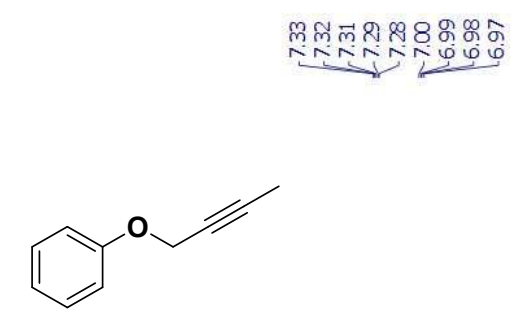

$\left(400 \mathrm{MHz}, \mathrm{CDCl}_{3}\right)$

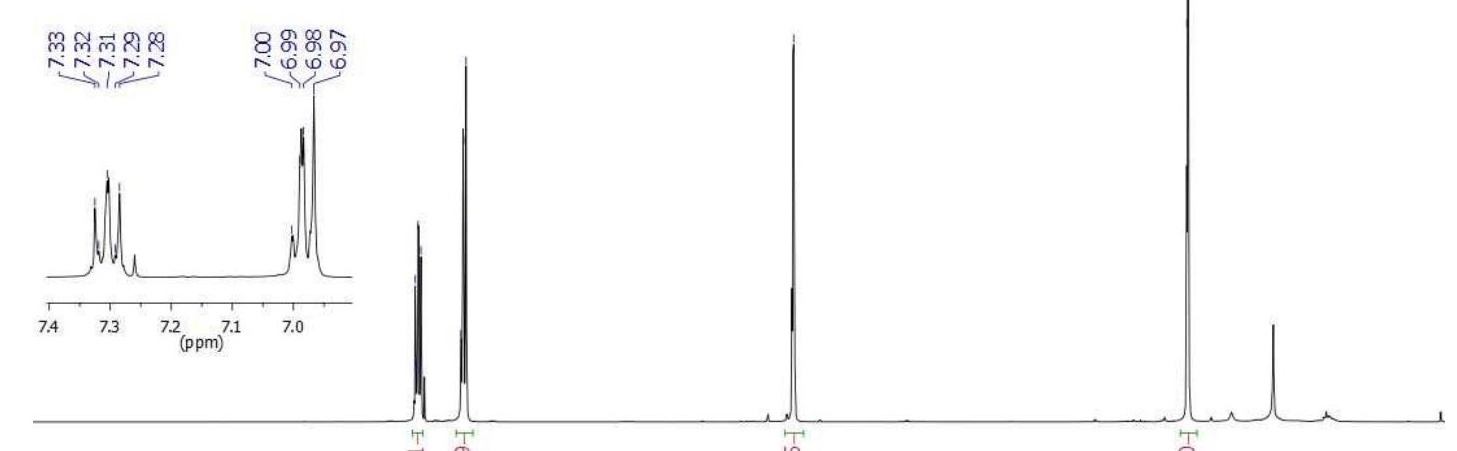


Figure S7. ${ }^{1} \mathrm{H}$ NMR of (but-2-yn-1-yloxy)benzene (1a).

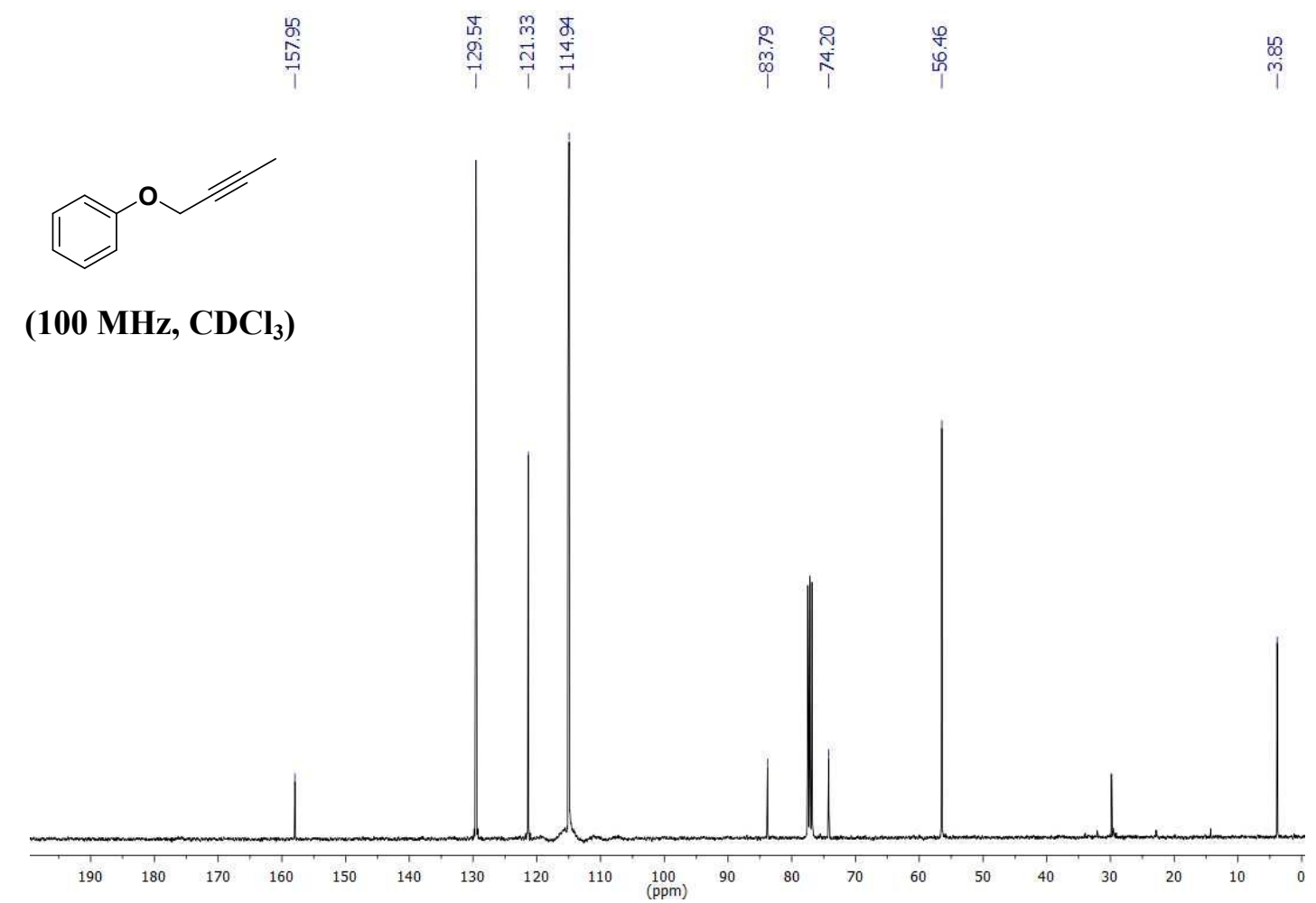

Figure S8. ${ }^{13} \mathrm{C}$ NMR of (but-2-yn-1-yloxy)benzene (1a).

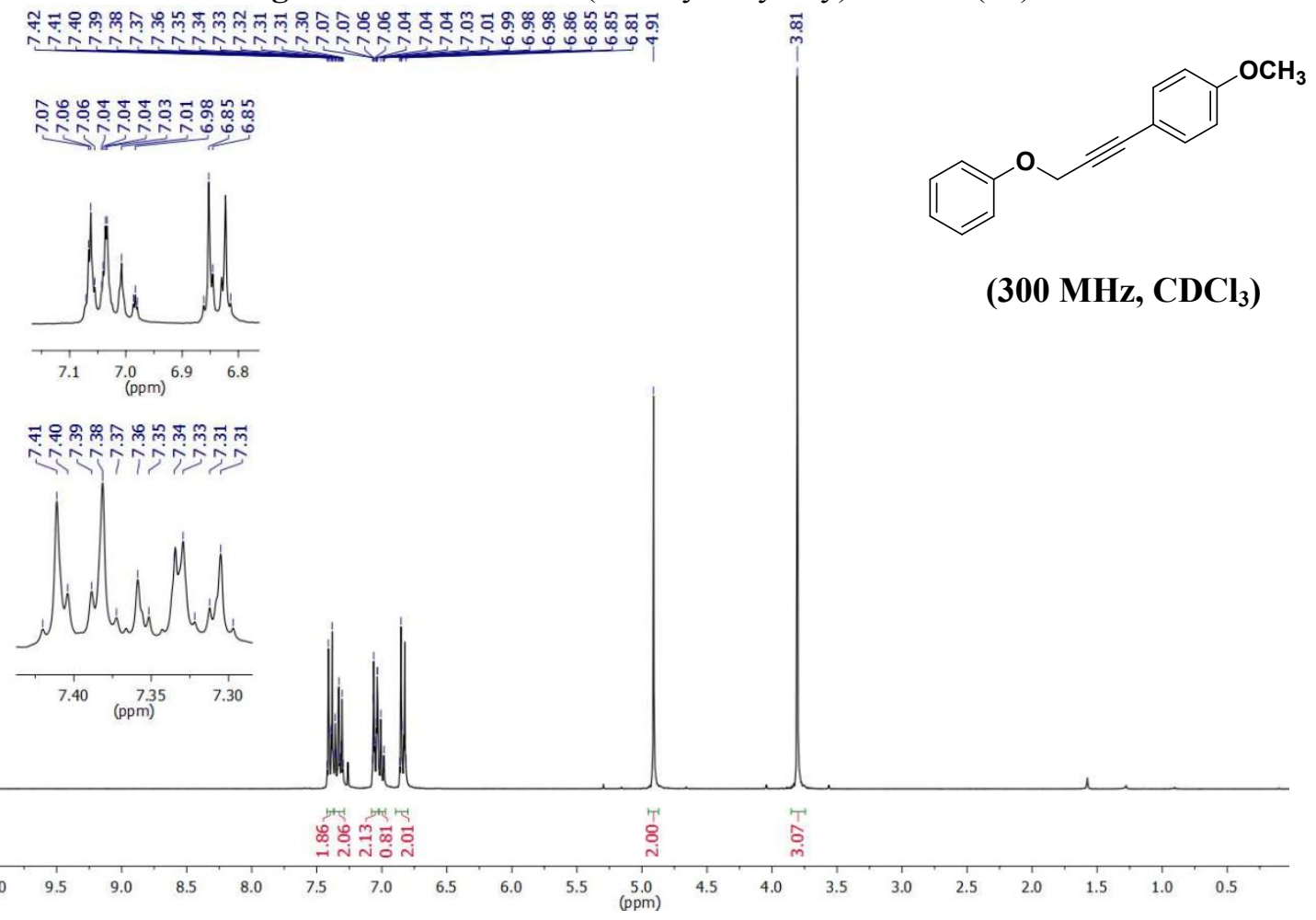


Figure S9. ${ }^{1} \mathrm{H}$ NMR of 1-methoxy-4-(3-phenoxyprop-1-yn-1-yl)benzene (2a).

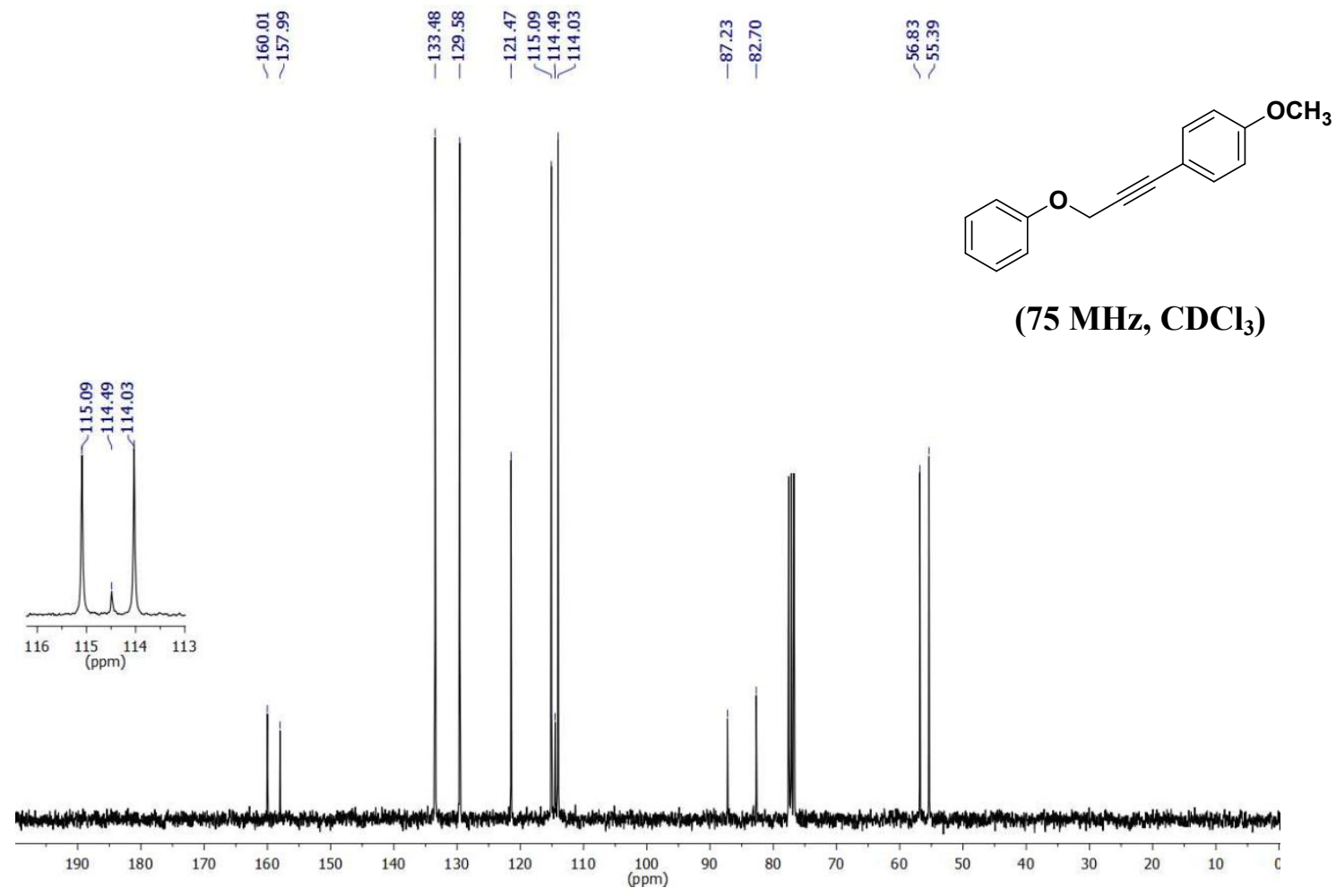

Figure S10. ${ }^{13} \mathrm{C}$ NMR of 1-methoxy-4-(3-phenoxyprop-1-yn-1-yl)benzene (2a).

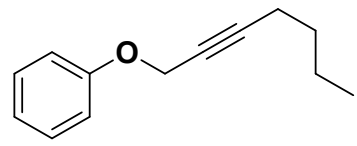

(300 MHz, $\left.\mathrm{CDCl}_{3}\right)$

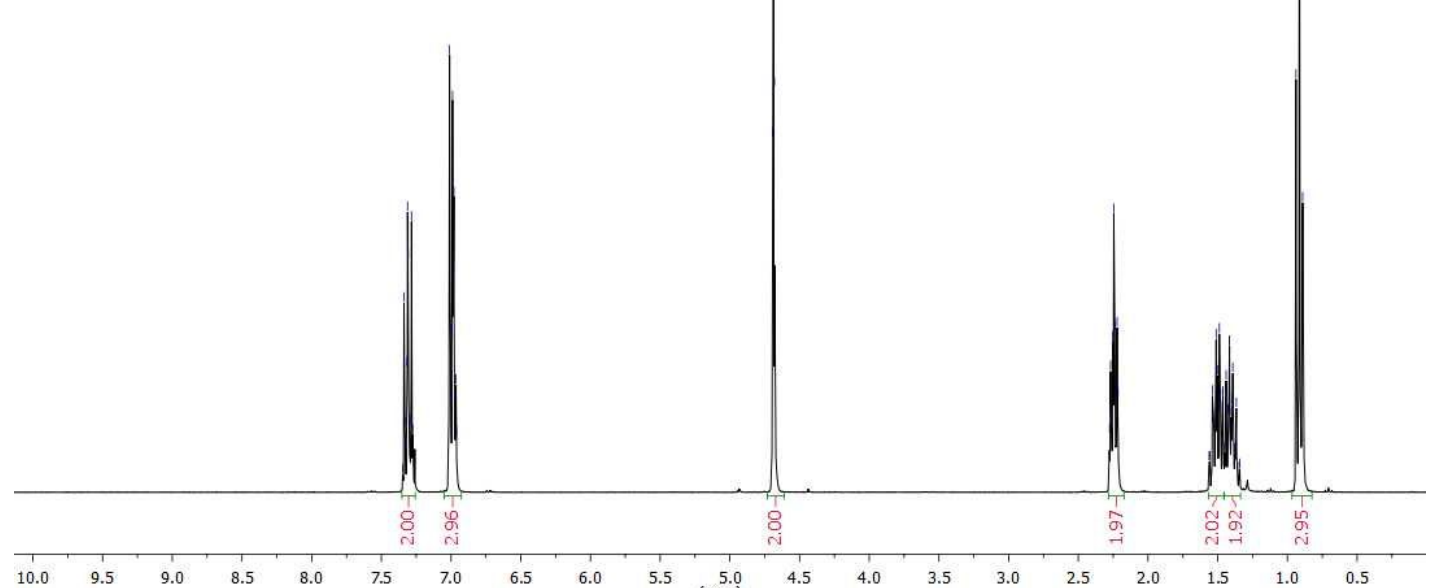


Figure S11. 'H NMR of (hept-2-yn-1-yloxy)benzene (3a).

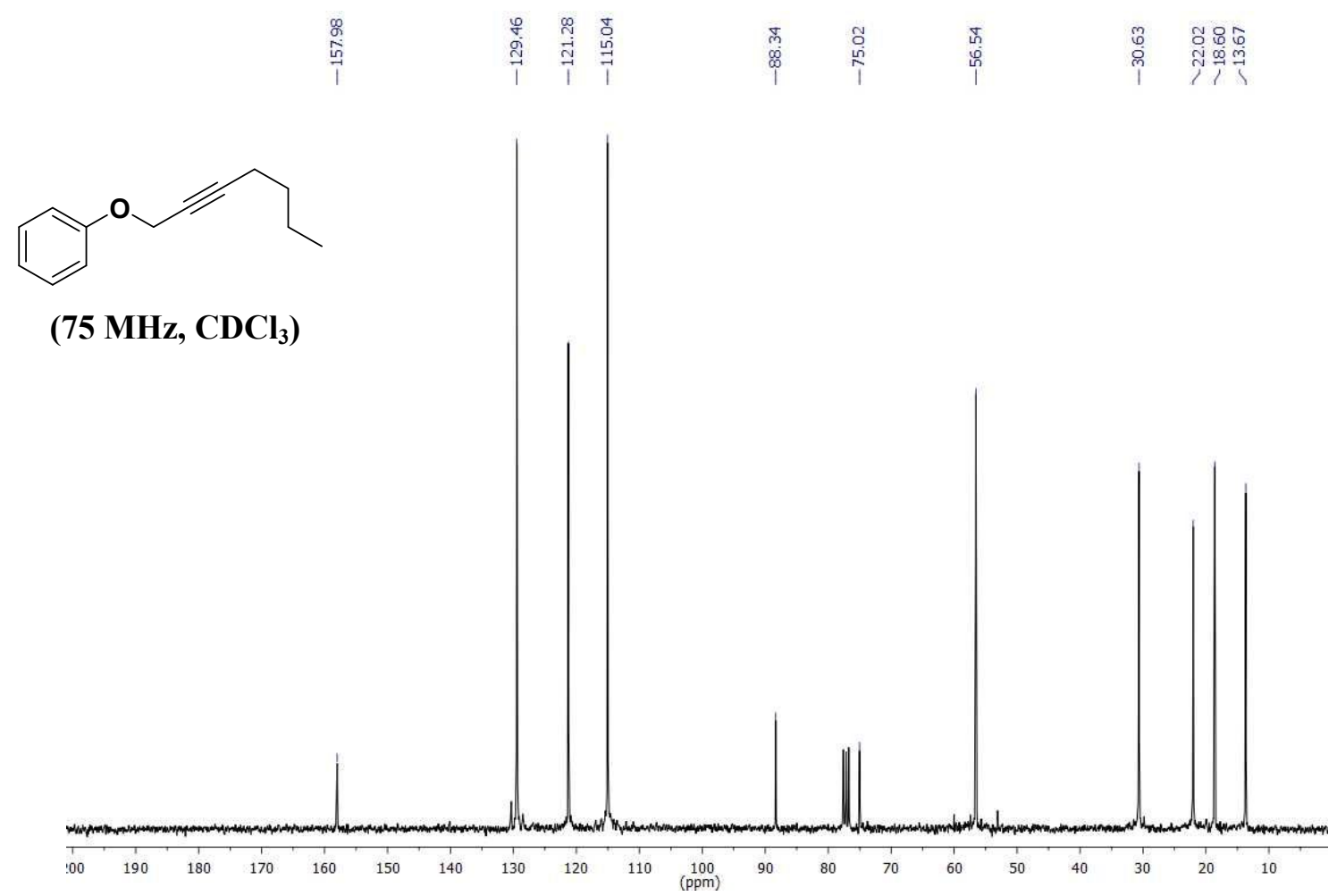

Figure S12. ${ }^{13} \mathrm{C}$ NMR of (hept-2-yn-1-yloxy)benzene (3a).

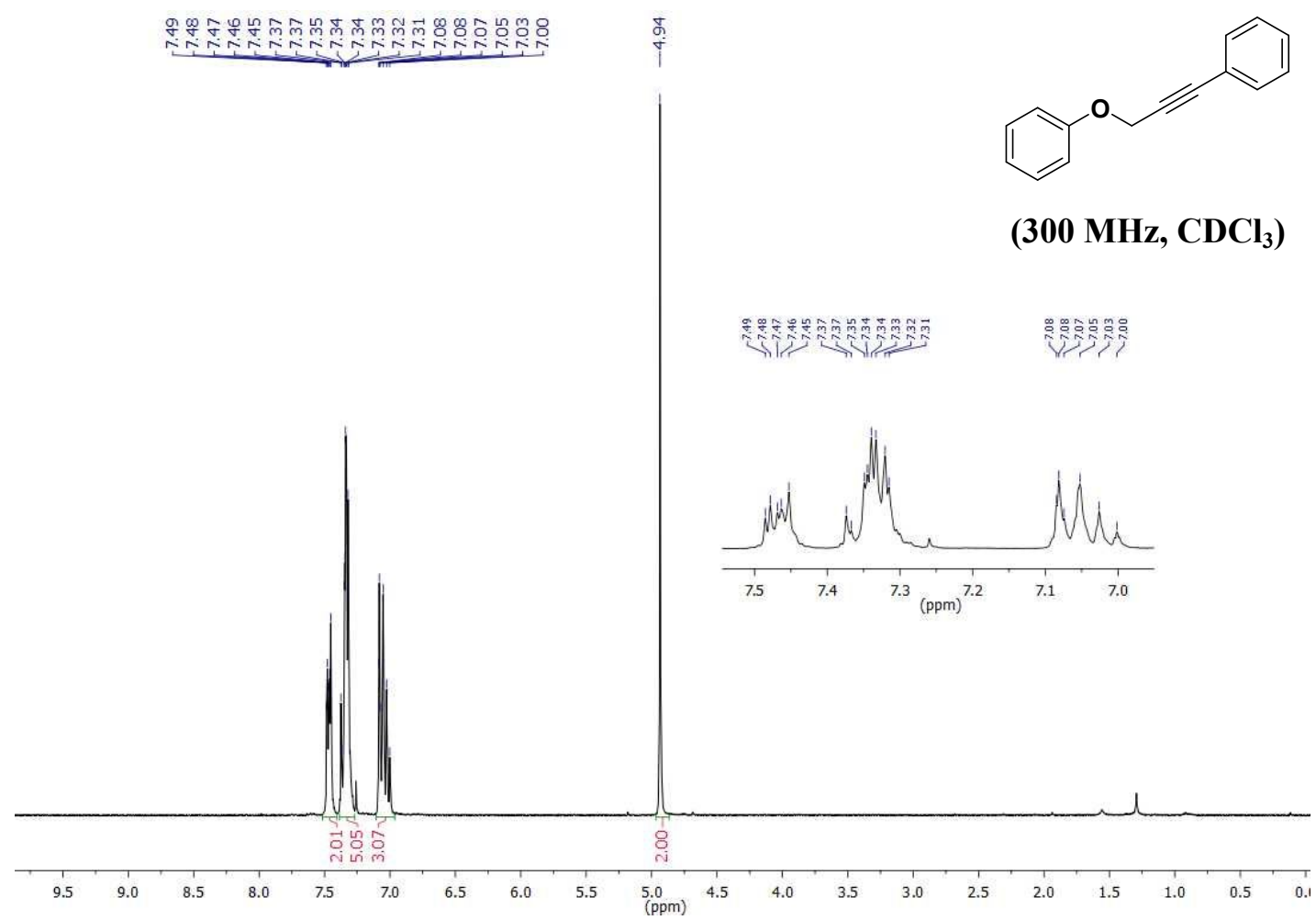


Figure S13. ${ }^{1} \mathrm{H}$ NMR of (3-phenoxyprop-1-yn-1-yl)benzene (4a).

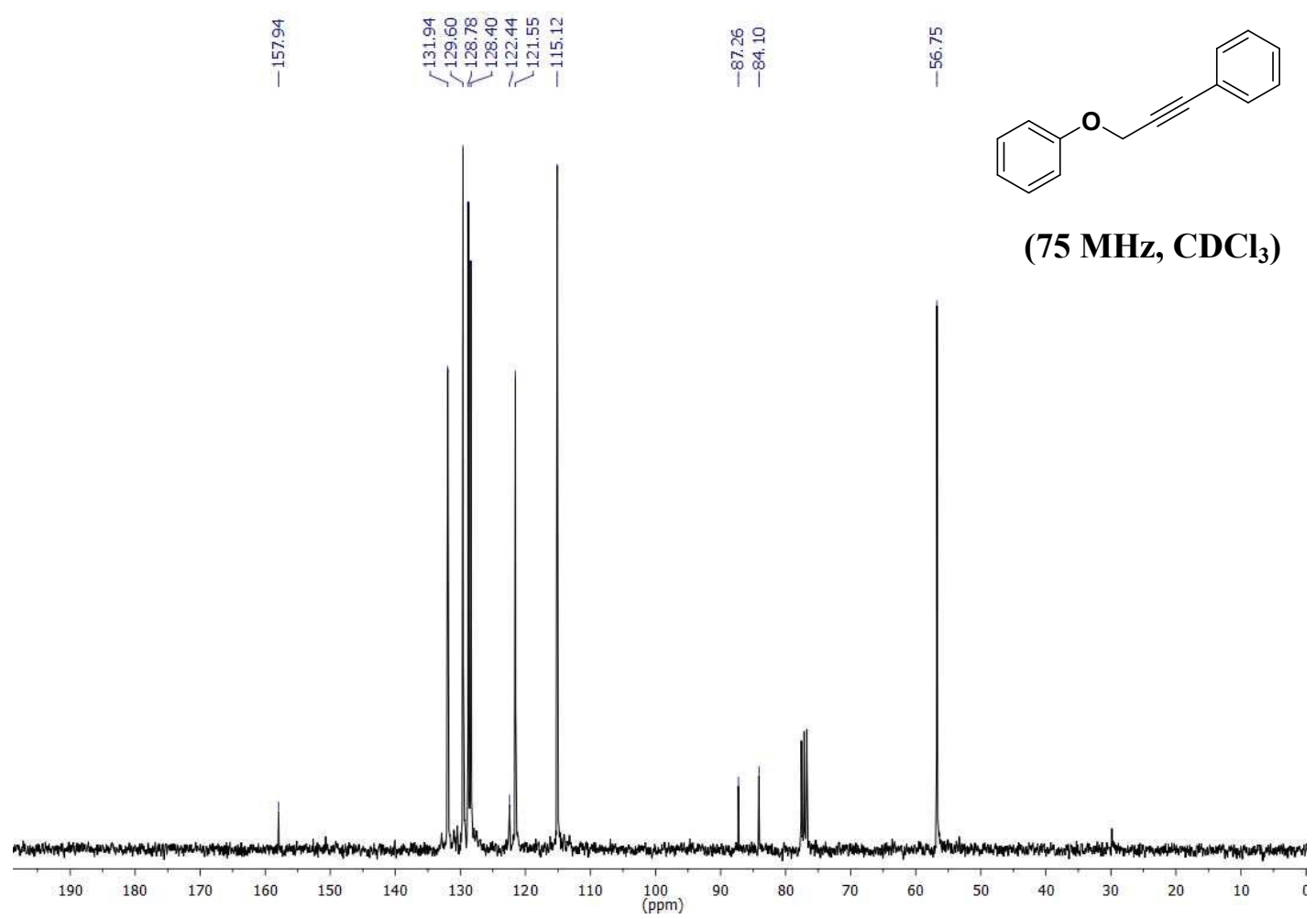

Figure S14. ${ }^{13} \mathrm{C}$ NMR of (3-phenoxyprop-1-yn-1-yl)benzene (4a).

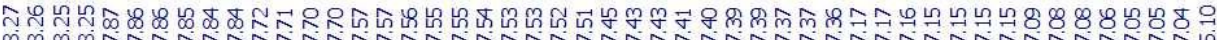
o

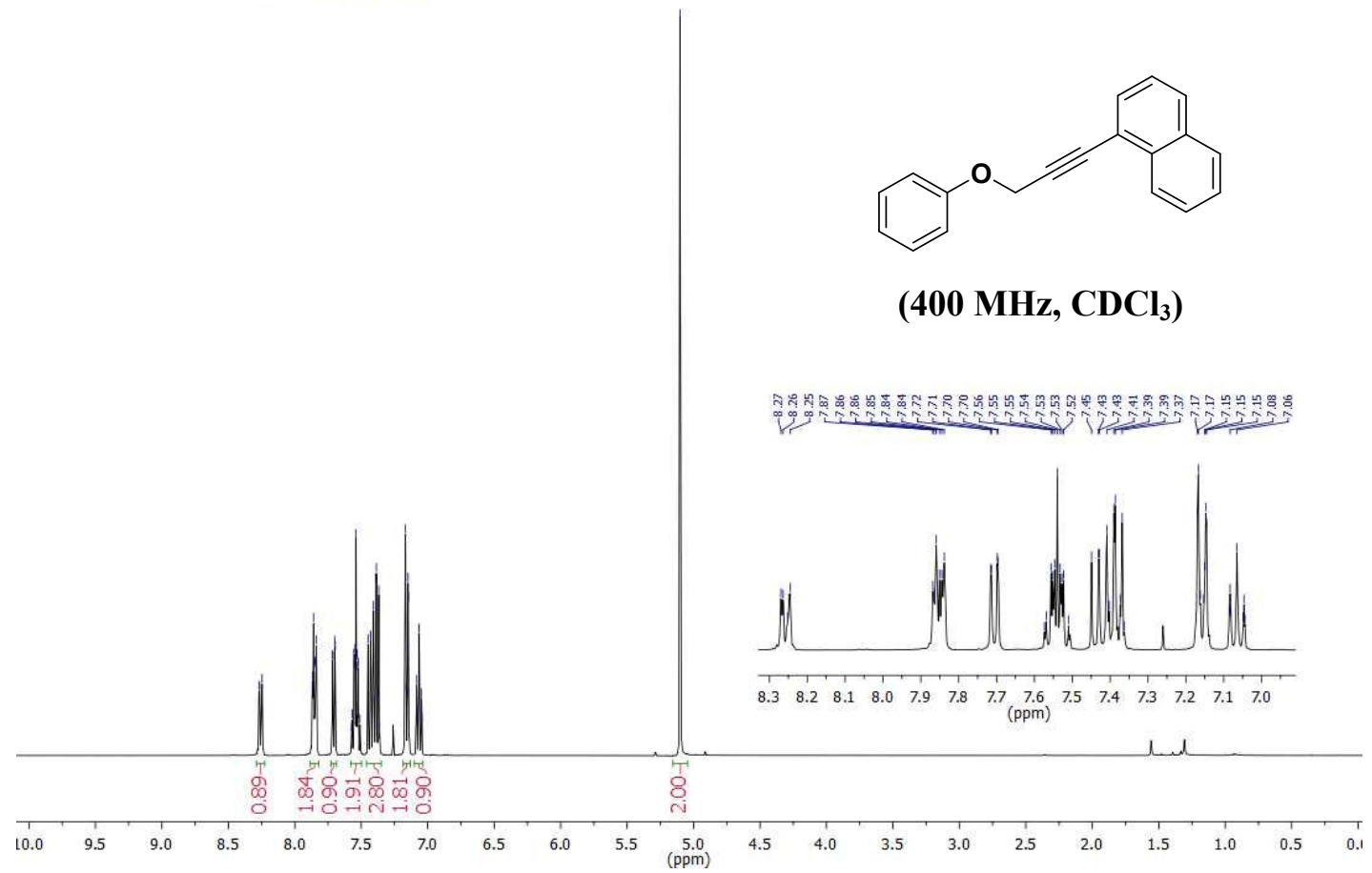


Figure S15. 'H NMR of 1-(3-Phenoxyprop-1-yn-1-yl)naphthalene (5a).

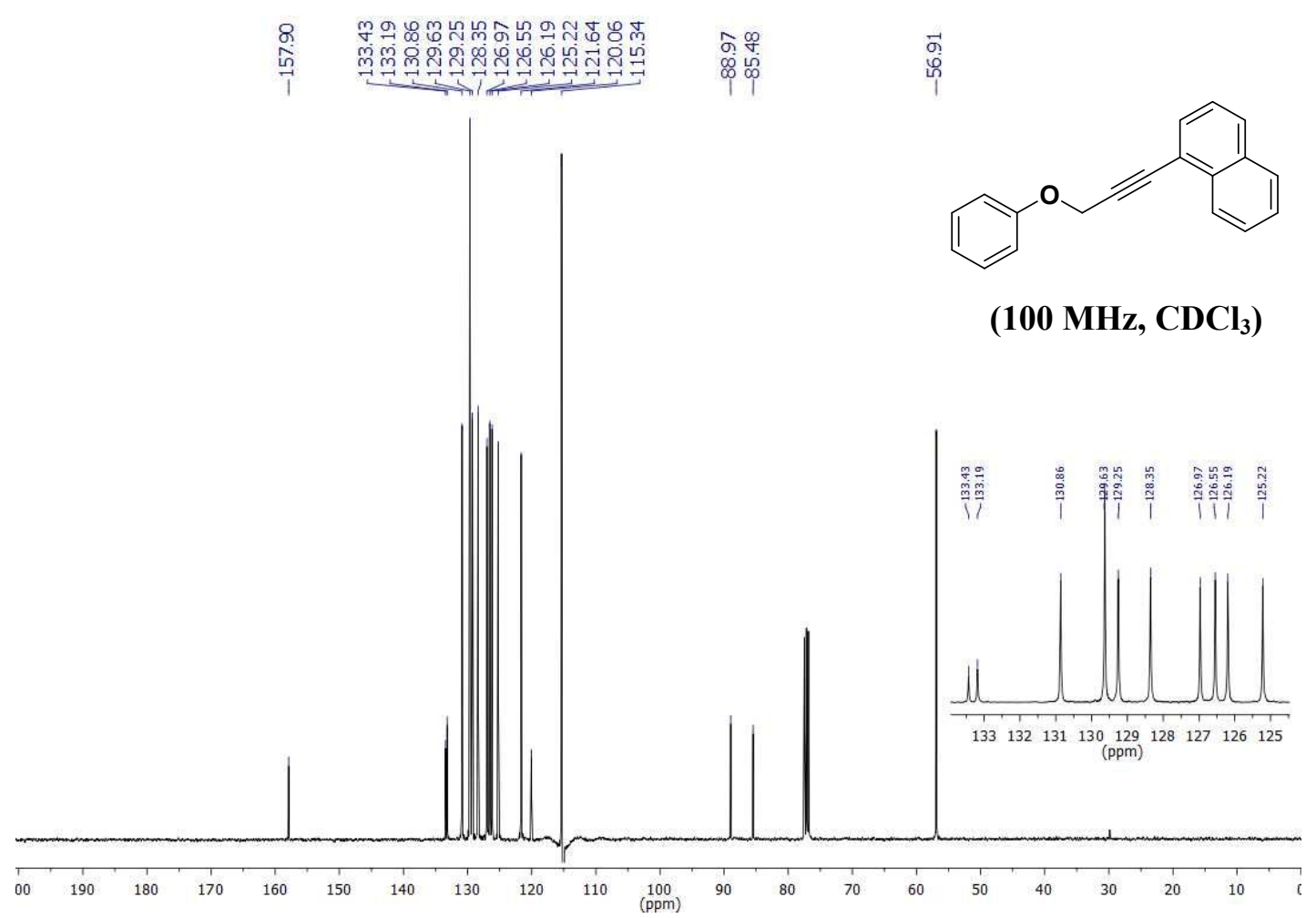

Figure S16. ${ }^{13} \mathrm{C}$ NMR of 1-(3-Phenoxyprop-1-yn-1-yl)naphthalene (5a).

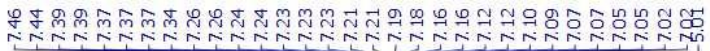
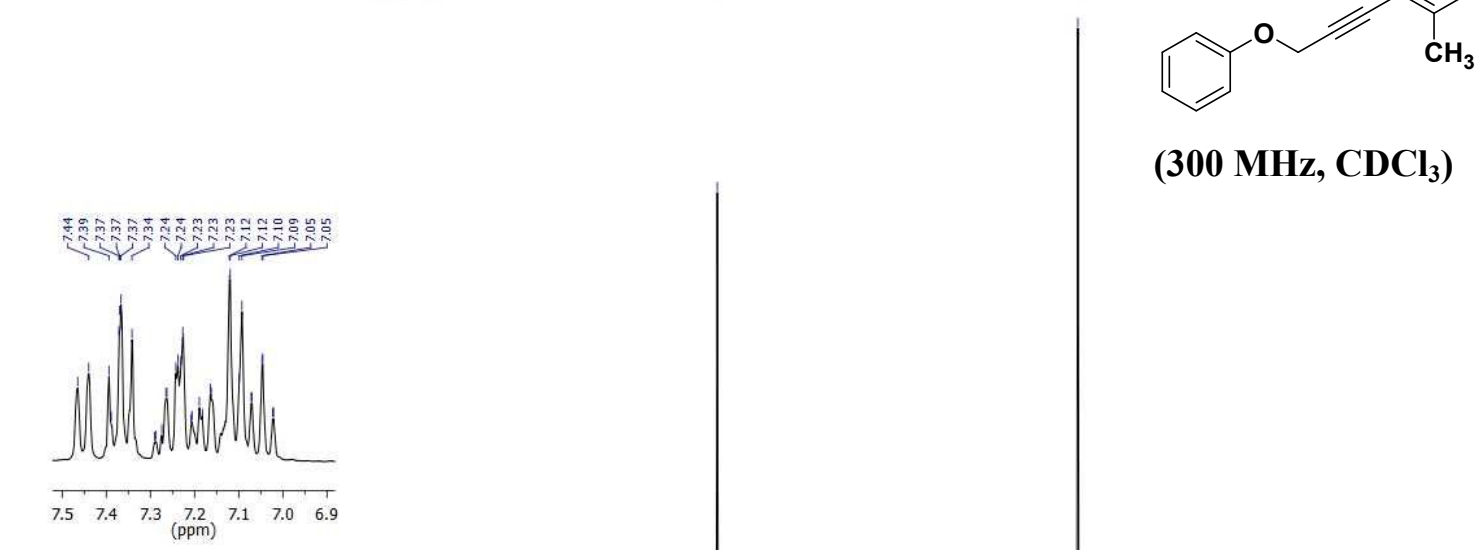

(300 MHz, $\left.\mathrm{CDCl}_{3}\right)$

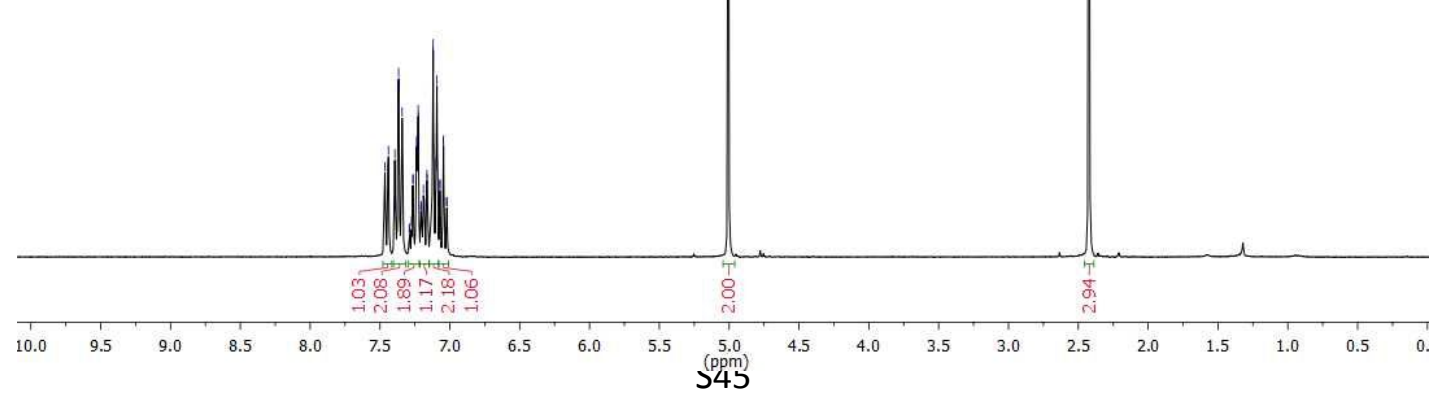


Figure S17. ${ }^{1} \mathrm{H}$ NMR of 1-methyl-2-(3-phenoxyprop-1-yn-1-yl)benzene (6a).

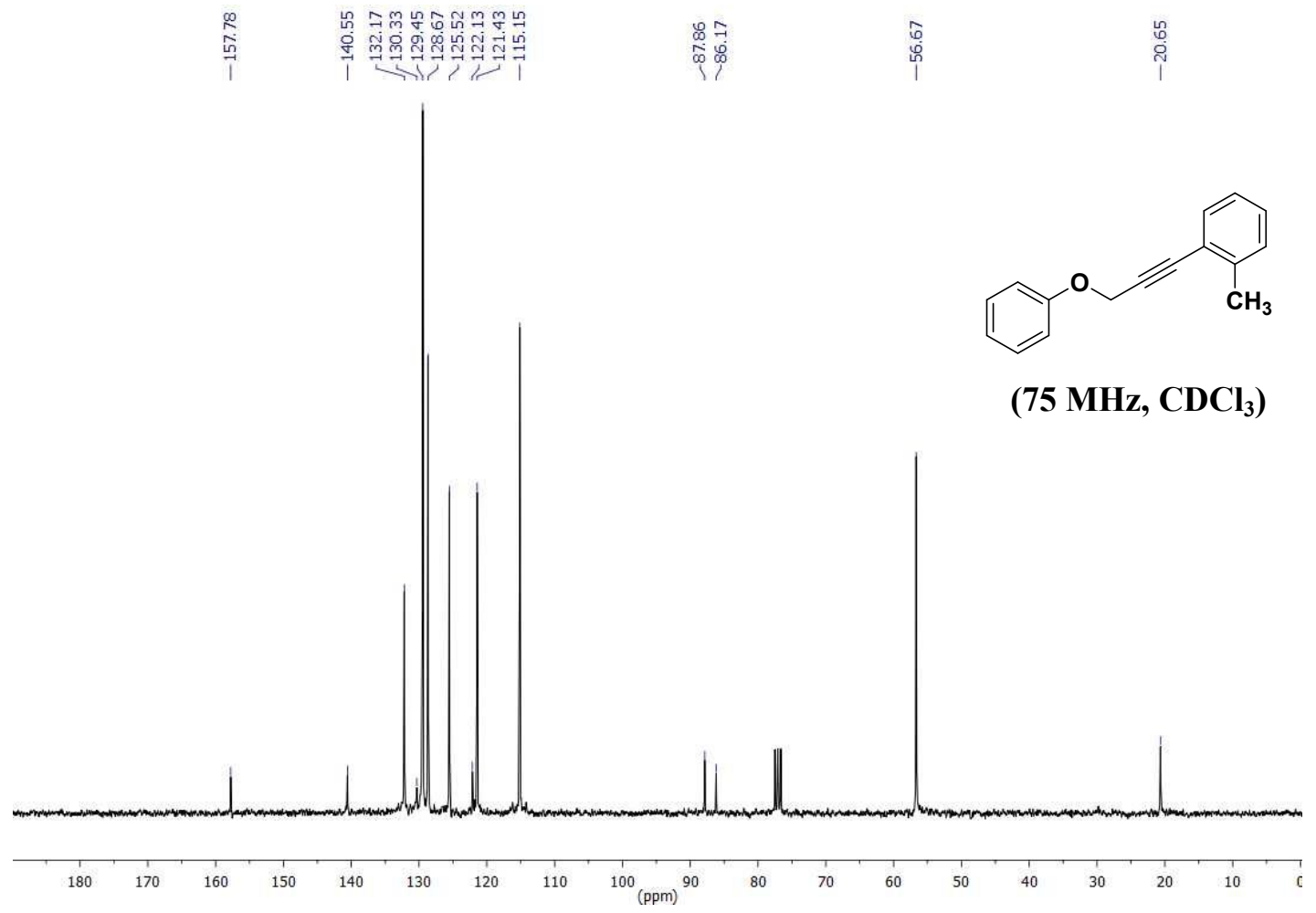

Figure S18. ${ }^{13} \mathrm{C}$ NMR of 1-methyl-2-(3-phenoxyprop-1-yn-1-yl)benzene (6a).

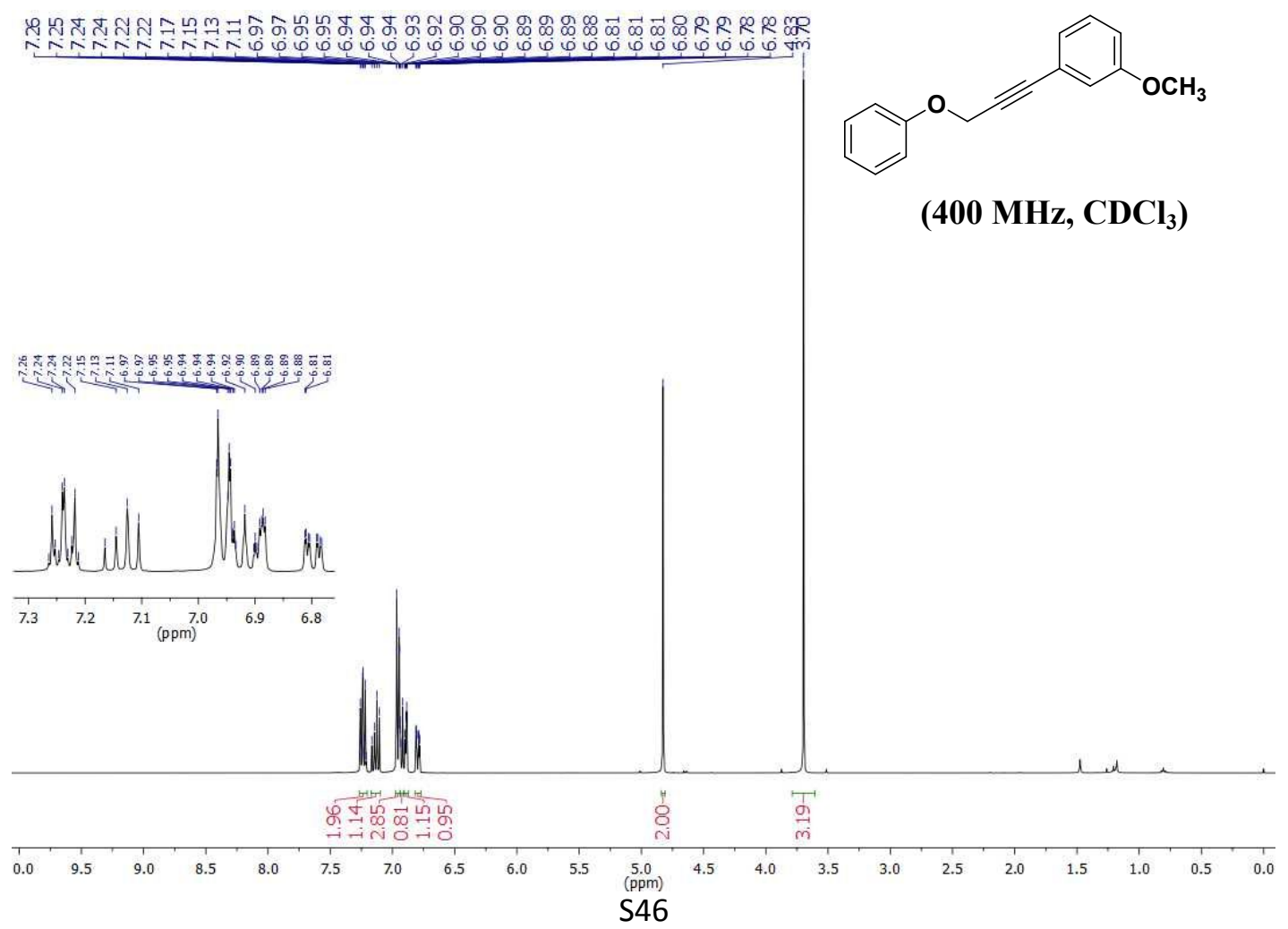


Figure S19. ${ }^{1} \mathrm{H}$ NMR of 1-Methoxy-3-(3-phenoxyprop-1-yn-1-yl)benzene (7a).

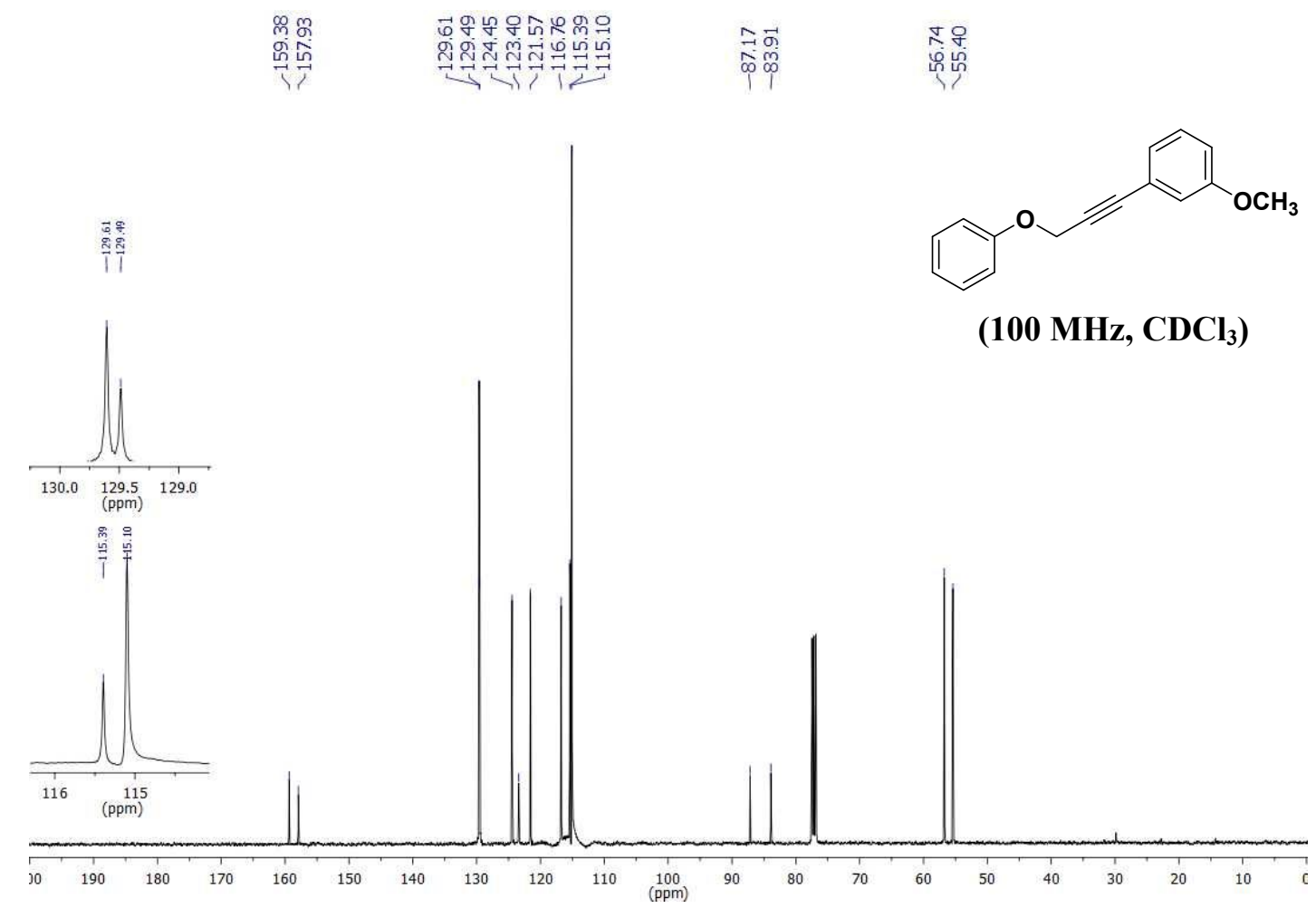

Figure S20. ${ }^{13} \mathrm{C}$ NMR of 1-Methoxy-3-(3-phenoxyprop-1-yn-1-yl)benzene (7a).

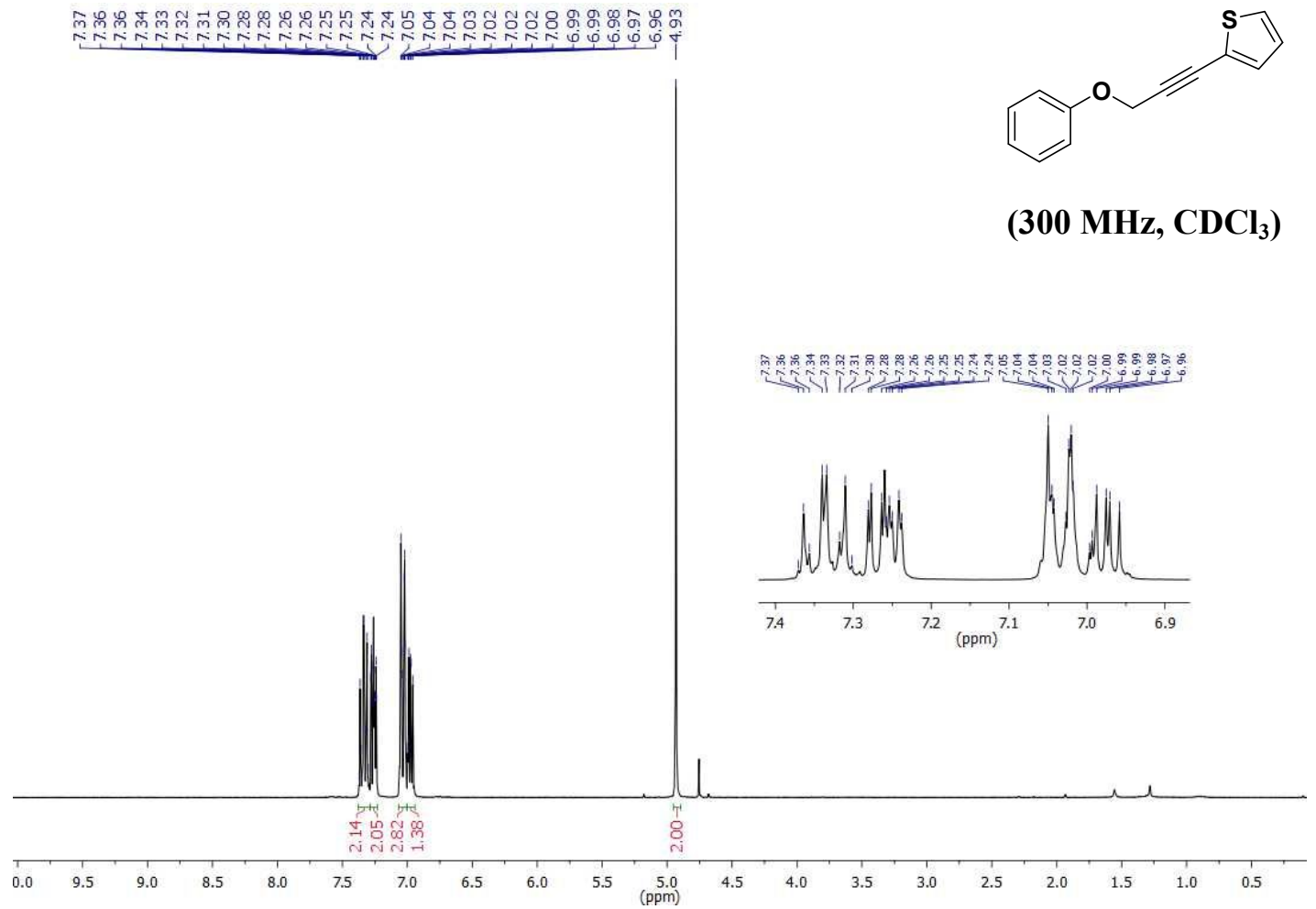


Figure S21. ${ }^{1} \mathrm{H}$ NMR of 2-(3-phenoxyprop-1-yn-1-yl)thiophene (9a).

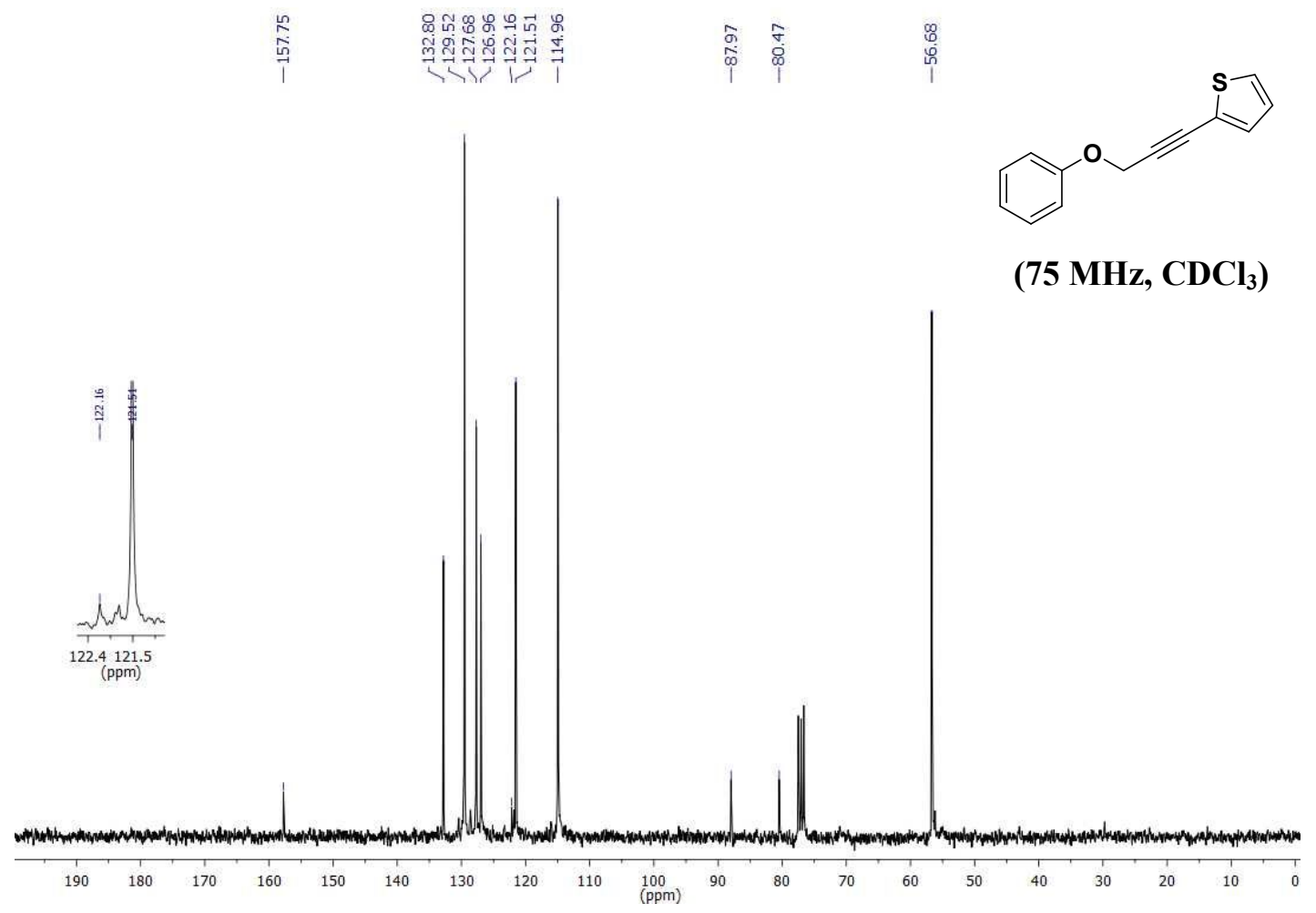

Figure S22. ${ }^{13} \mathrm{C}$ NMR of 2-(3-phenoxyprop-1-yn-1-yl)thiophene (9a).

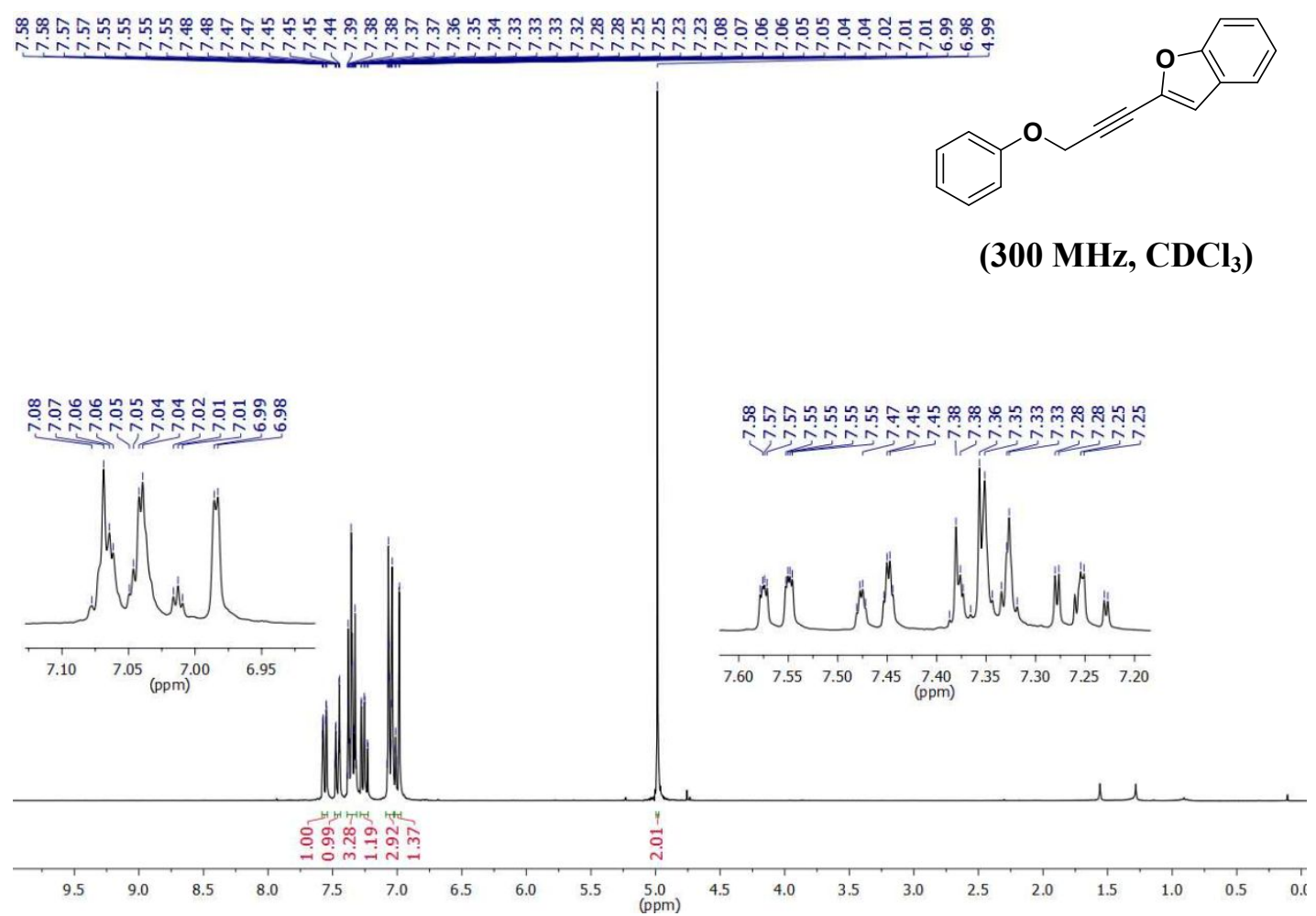

Figure S23. ${ }^{1} \mathrm{H}$ NMR of 2-(3-phenoxyprop-1-yn-1-yl)benzofuran (10a). 


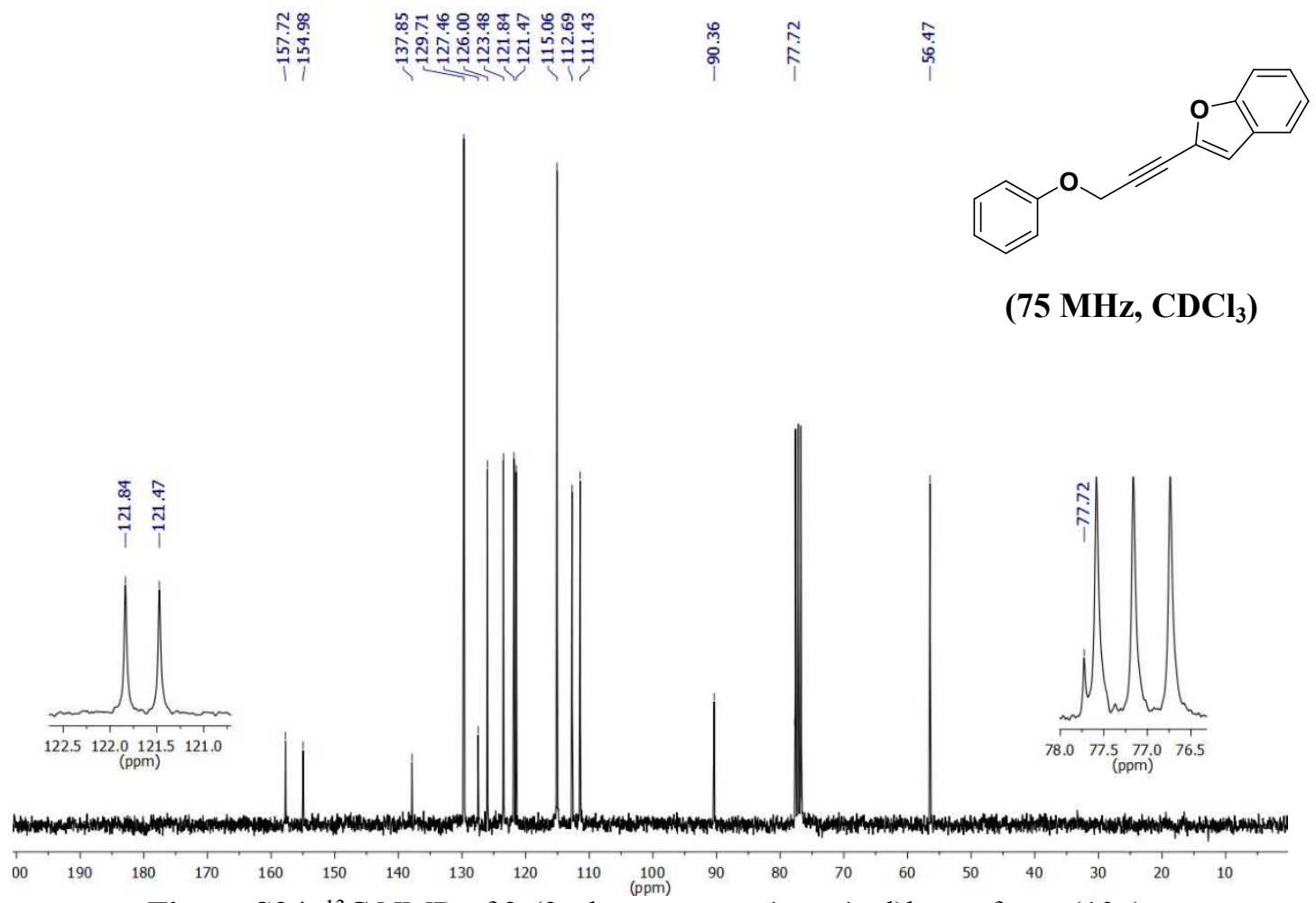

Figure S24. ${ }^{13} \mathrm{C}$ NMR of 2-(3-phenoxyprop-1-yn-1-yl)benzofuran (10a).

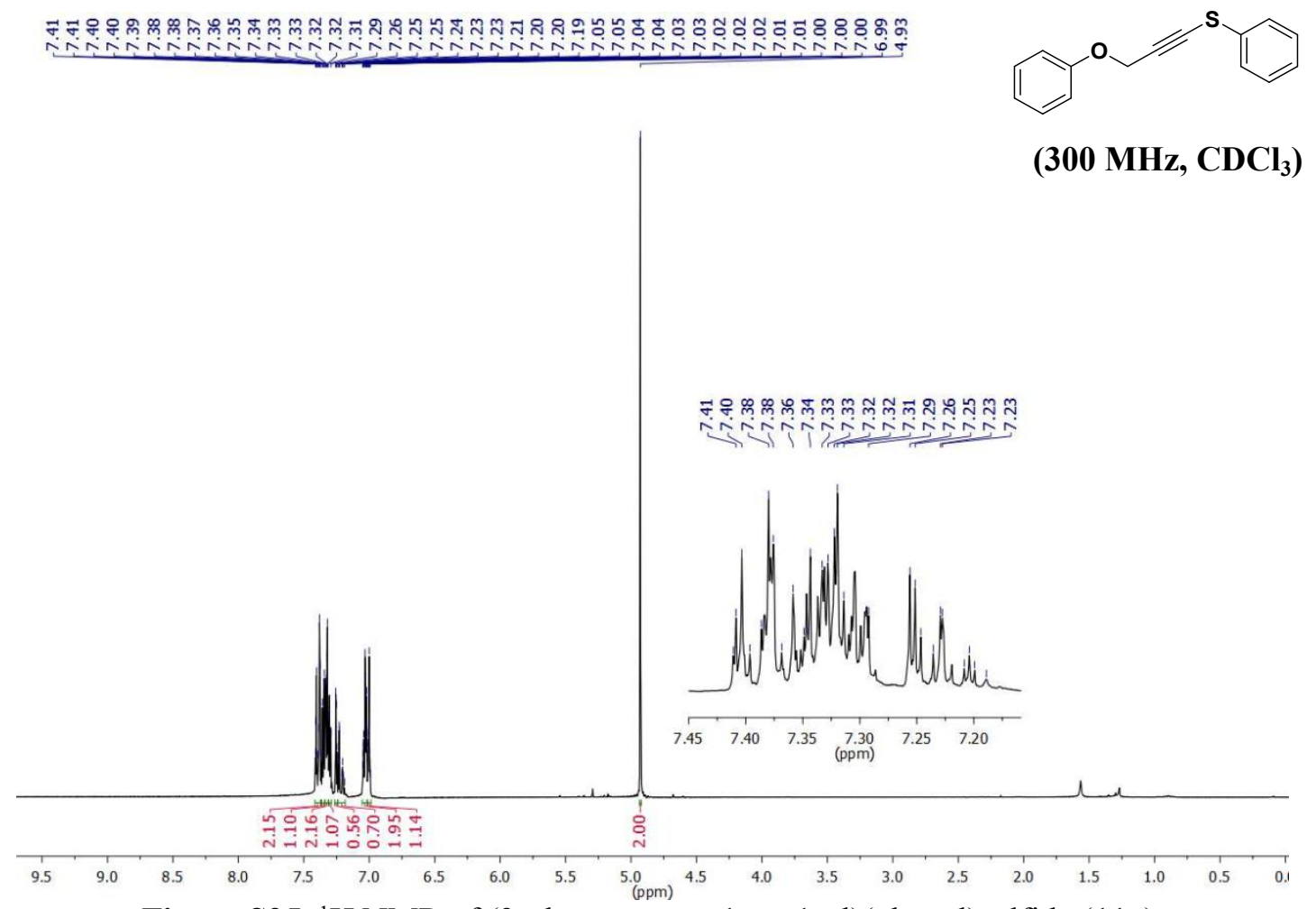

Figure S25. ${ }^{1} \mathrm{H}$ NMR of (3-phenoxyprop-1-yn-1-yl)(phenyl)sulfide (11a). 


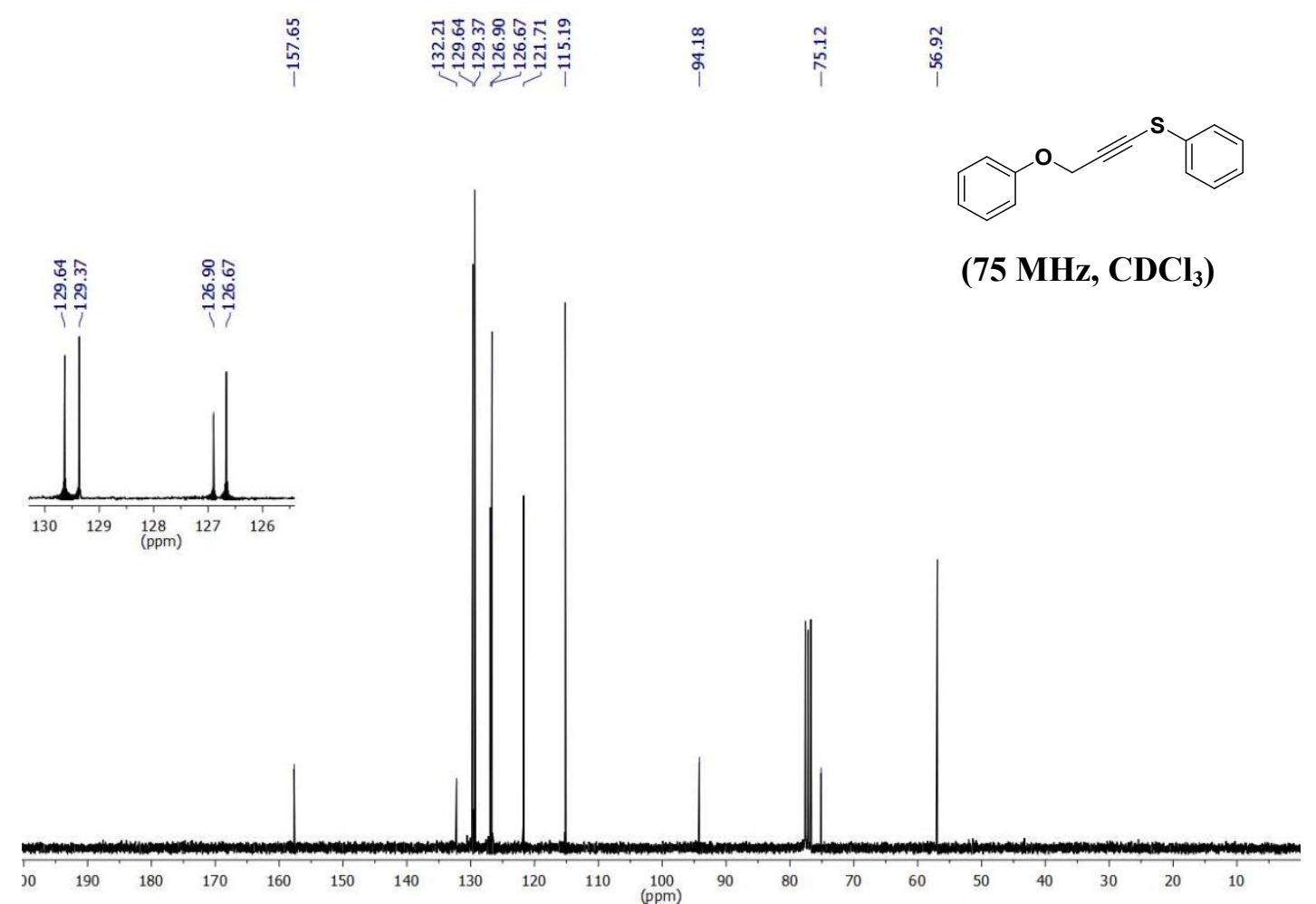

Figure S26. ${ }^{13} \mathrm{C}$ NMR of (3-phenoxyprop-1-yn-1-yl)(phenyl)sulfide (11a).

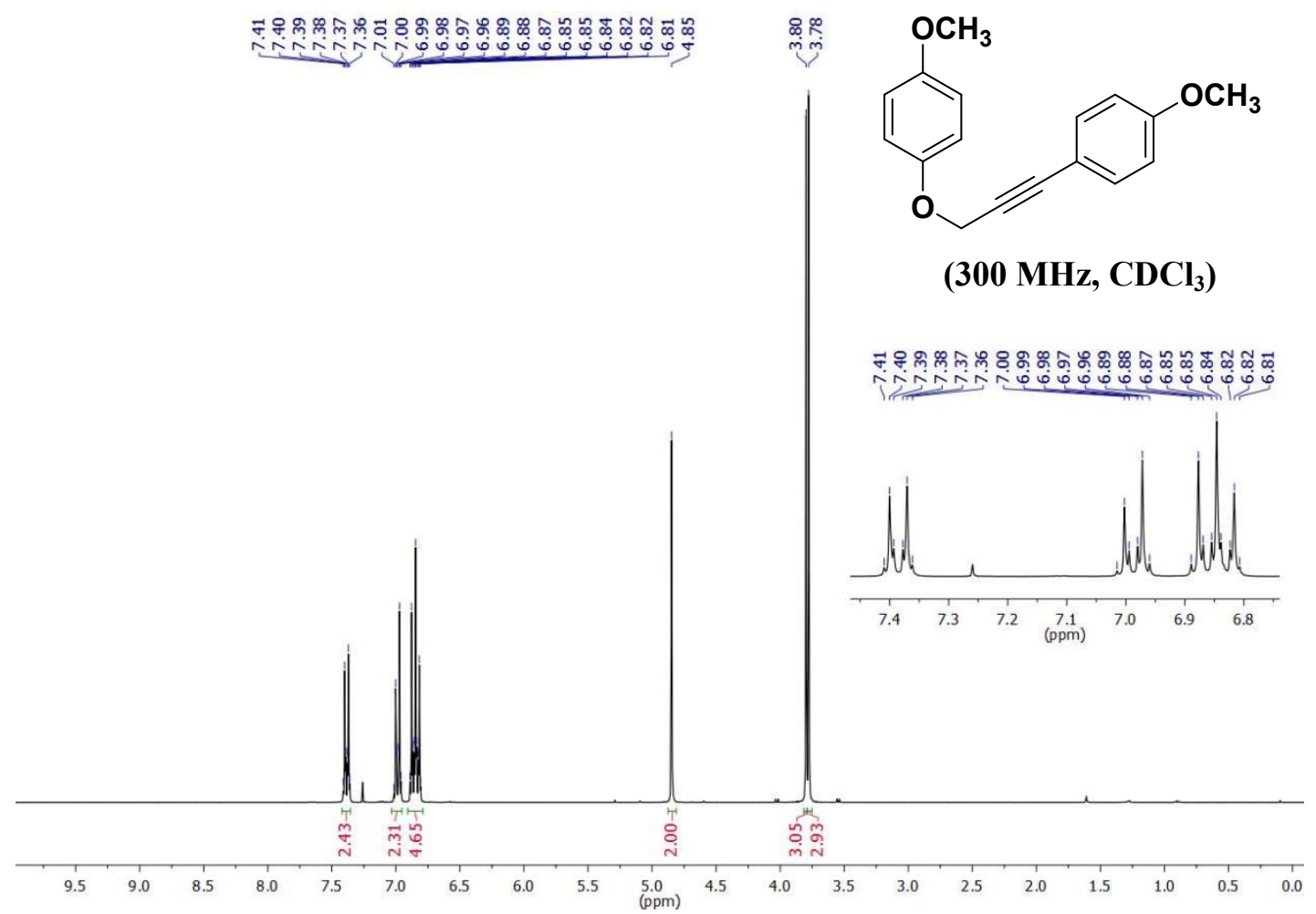

Figure S27. ${ }^{1} \mathrm{H}$ NMR of 1-methoxy-4-(3-(4-methoxyphenoxy)prop-1-yn-1-yl)benzene (13a).

\begin{tabular}{|c|c|c|c|}
\hline 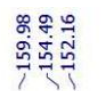 & $\begin{array}{l}m \\
\stackrel{m}{m} \\
\stackrel{m}{p}\end{array}$ & 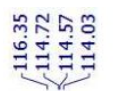 & 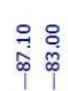 \\
\hline
\end{tabular}




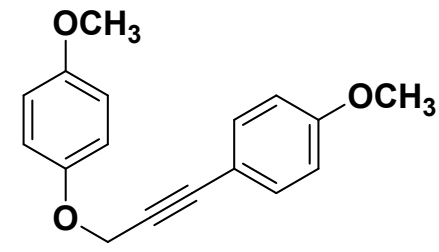

(75 MHz, $\mathrm{CDCl}_{3}$ )

Figure S28. ${ }^{13} \mathrm{C}$ NMR of 1-methoxy-4-(3-(4-methoxyphenoxy)prop-1-yn-1-yl)benzene (13a).

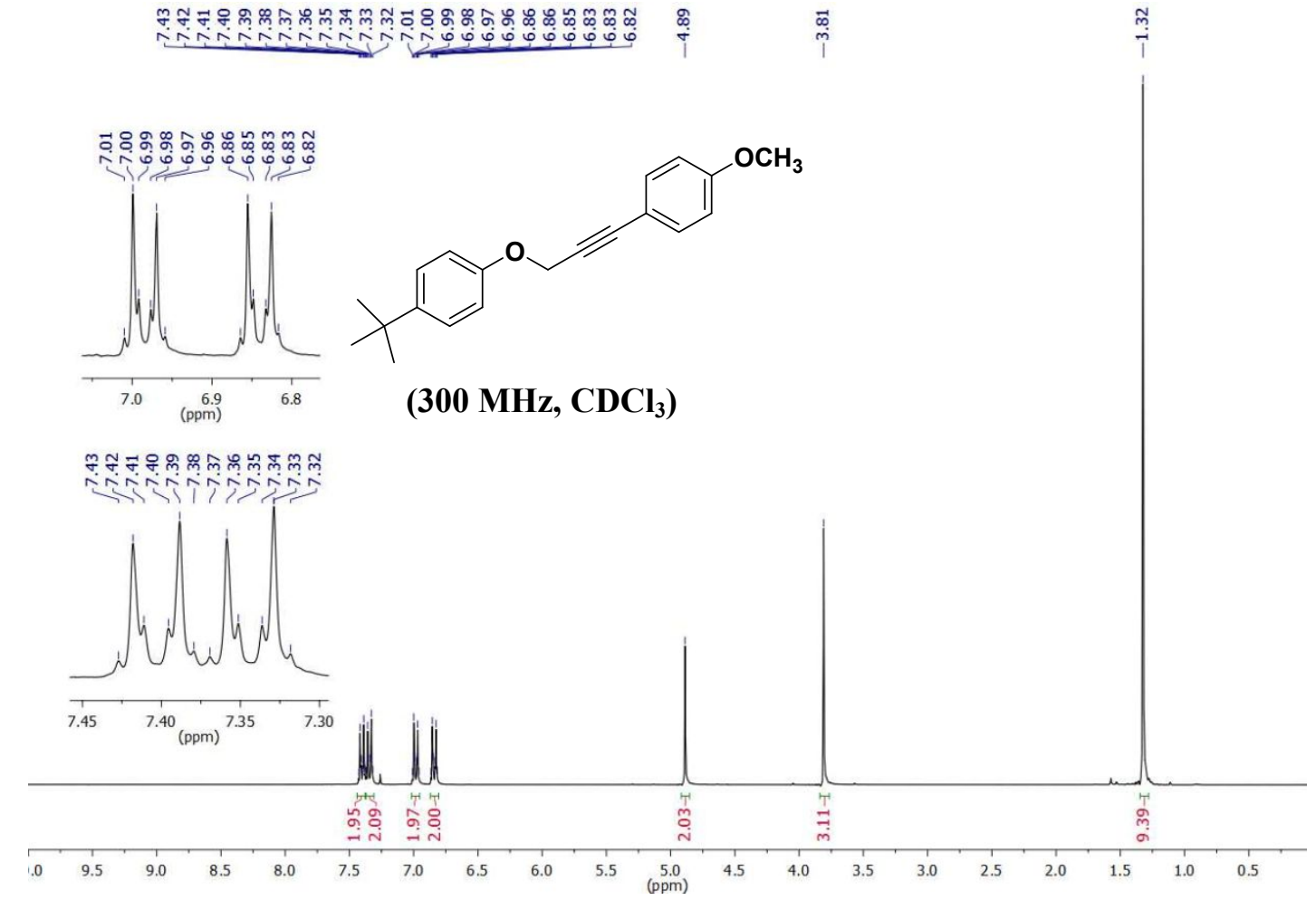

Figure S29. ${ }^{1} \mathrm{H}$ NMR of 1-(tert-butyl)-4-((3-(4-methoxyphenyl)prop-2-yn-1-yl)oxy)benzene (14a).

\begin{tabular}{|c|c|c|c|c|c|c|c|}
\hline 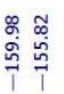 & $\begin{array}{l}\stackrel{0}{+} \\
\dot{\sigma} \\
+1\end{array}$ & $\begin{array}{l}\frac{g}{p} \\
\stackrel{m}{r}\end{array}$ & 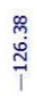 & 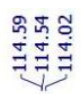 & 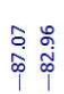 & 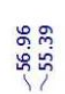 & 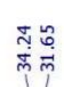 \\
\hline
\end{tabular}




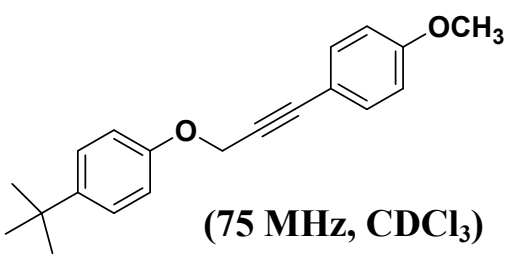

Figure S30. ${ }^{13} \mathrm{C}$ NMR of 1-(tert-butyl)-4-((3-(4-methoxyphenyl)prop-2-yn-1-yl)oxy)benzene (14a).

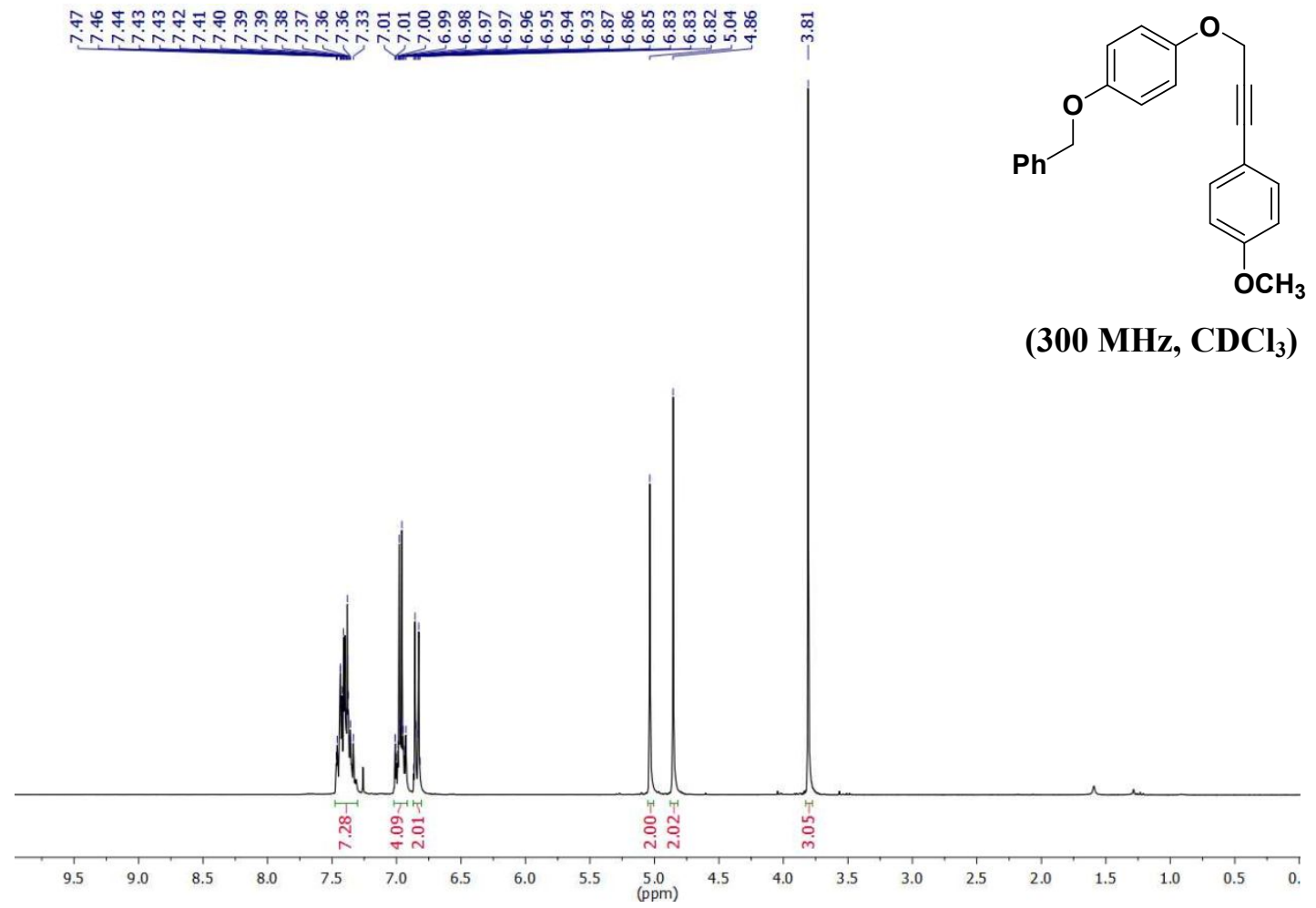

Figure S31. 'H NMR of 1-(benzyloxy)-4-((3-(4-methoxyphenyl)prop-2-yn-1-yl)oxy)benzene (15a).

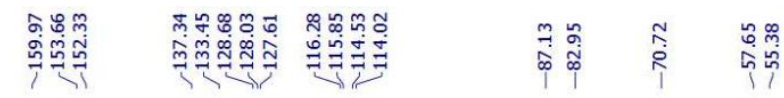
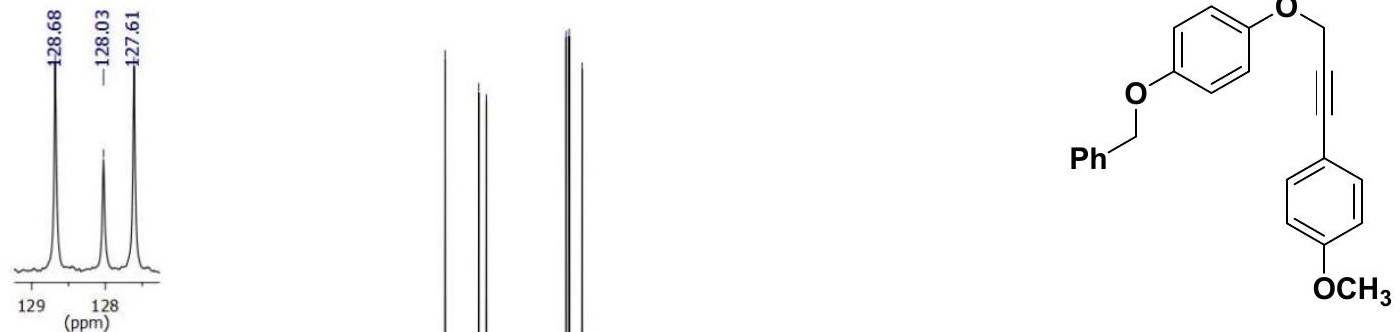
(75 $\mathrm{MHz}, \mathrm{CDCl}_{3}$ )

Figure S32. ${ }^{13} \mathrm{C}$ NMR of 1-(benzyloxy)-4-((3-(4-methoxyphenyl)prop-2-yn-1-yl)oxy)benzene (15a).

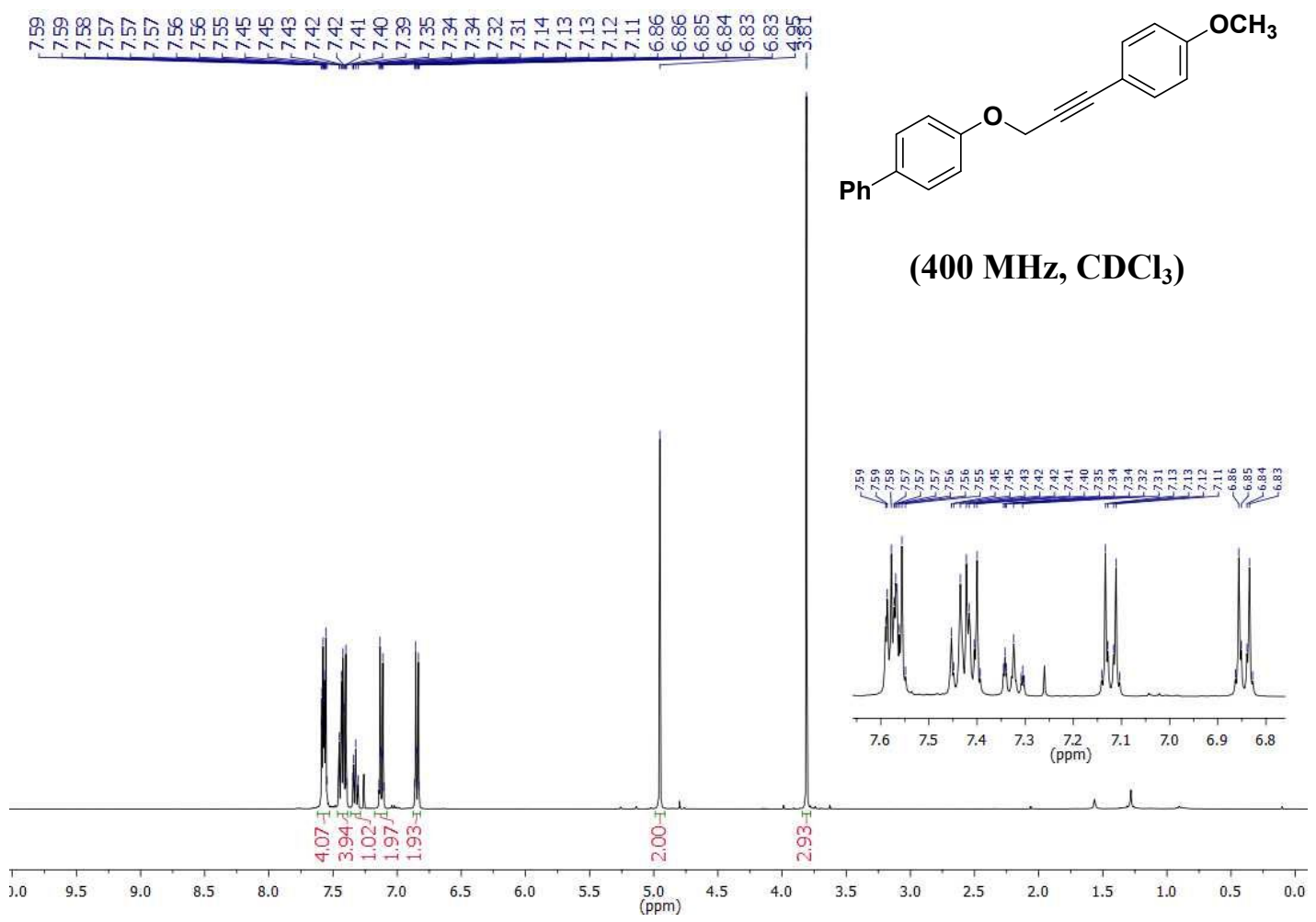

Figure S33. ${ }^{1} \mathrm{H}$ NMR of 4-((3-(4-Methoxyphenyl)prop-2-yn-1-yl)oxy)-1,1'-biphenyl (16a).

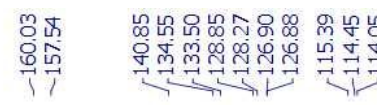

$\underset{\substack { 0 \\ \begin{subarray}{c}{0 \\ \infty{ 0 \\ \begin{subarray} { c } { 0 \\ \infty } }\end{subarray}}{\substack{0 \\ \infty}}$

홍ํำ

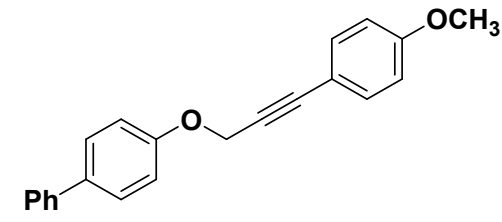


$\left(100 \mathrm{MHz}, \mathrm{CDCl}_{3}\right)$

Figure S34. ${ }^{13} \mathrm{C}$ NMR of 4-((3-(4-Methoxyphenyl)prop-2-yn-1-yl)oxy)-1,1'-biphenyl (16a).

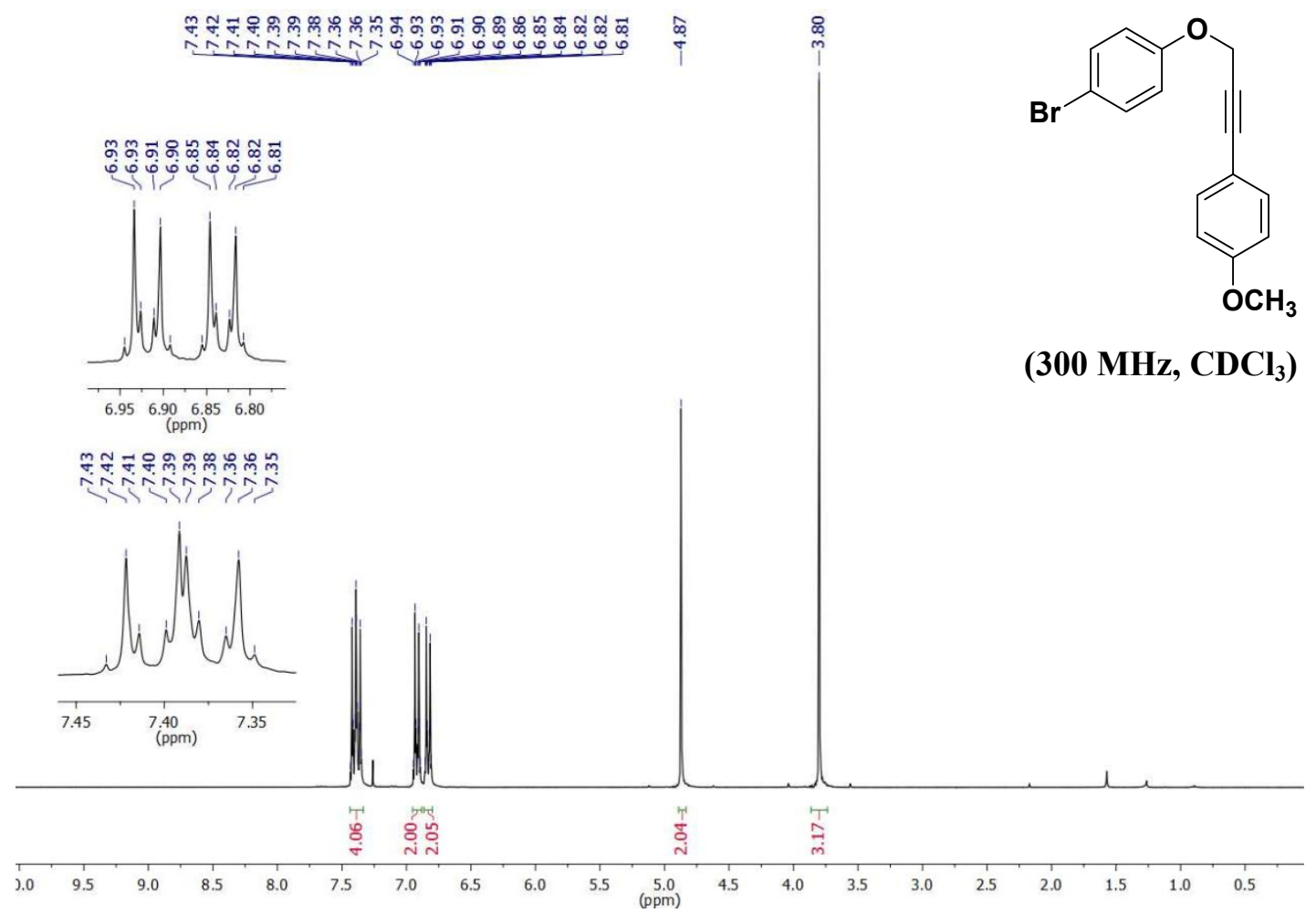

Figure S35. ${ }^{1} \mathrm{H}$ NMR of 1-bromo-4-((3-(4-methoxyphenyl)prop-2-yn-1-yl)oxy)benzene (17a).

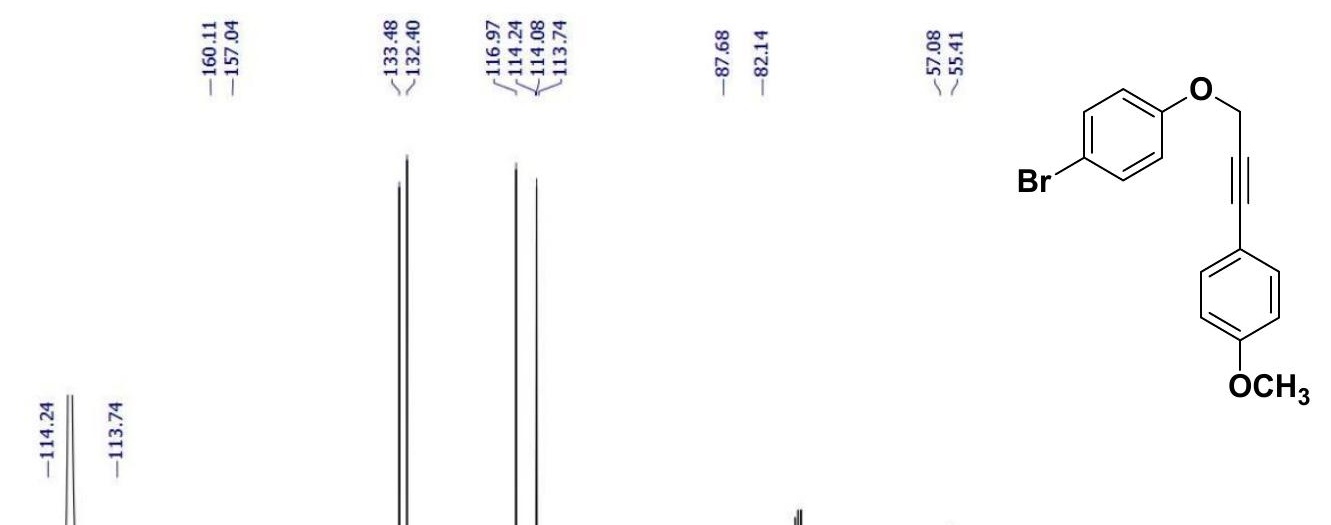


(75 $\left.\mathrm{MHz}, \mathrm{CDCl}_{3}\right)$

Figure S36. ${ }^{13} \mathrm{C}$ NMR of 1-bromo-4-((3-(4-methoxyphenyl)prop-2-yn-1-yl)oxy)benzene (17a).

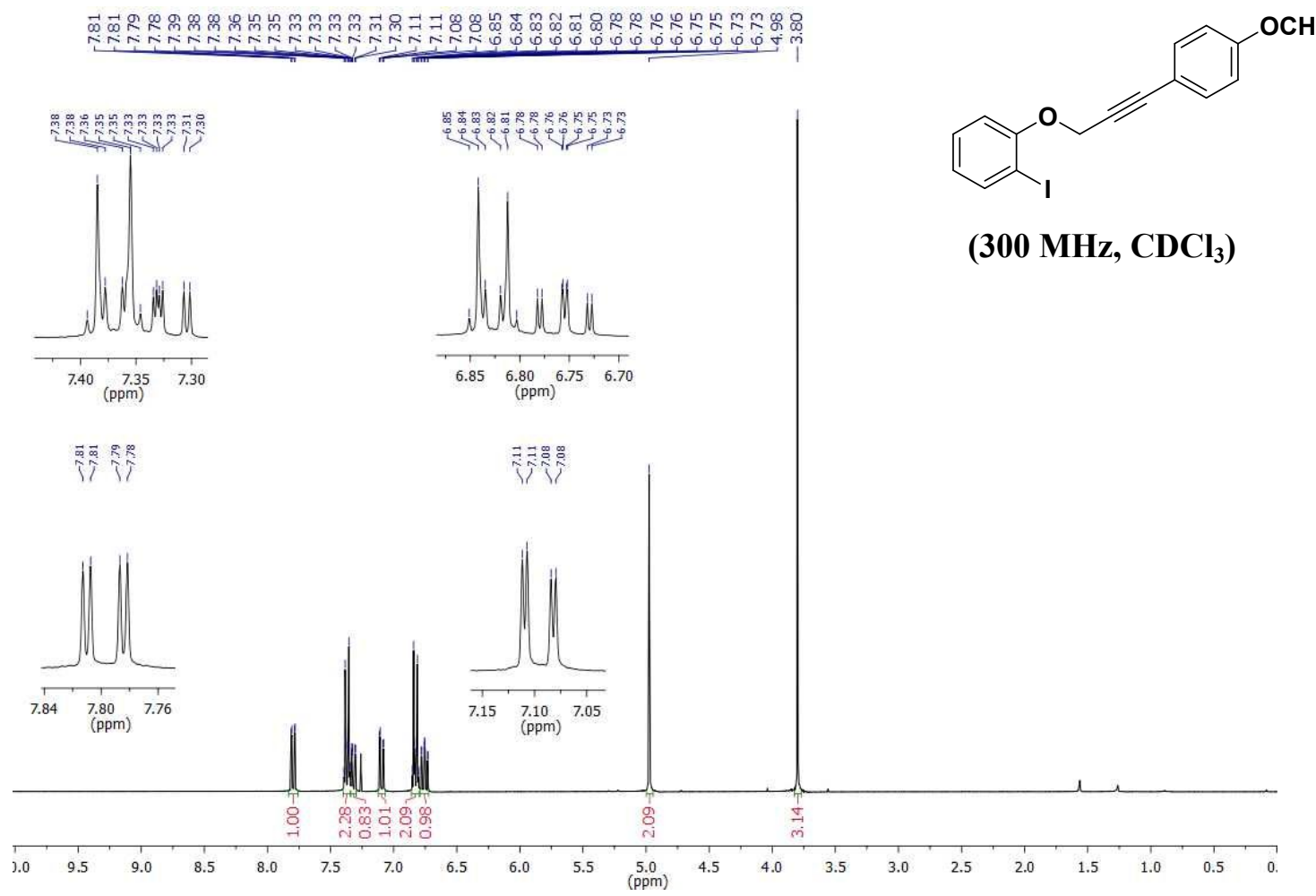

Figure S37. ${ }^{1} \mathrm{H}$ NMR of 1-iodo-2-((3-(4-methoxyphenyl)prop-2-yn-1-yl)oxy)benzene (18a).

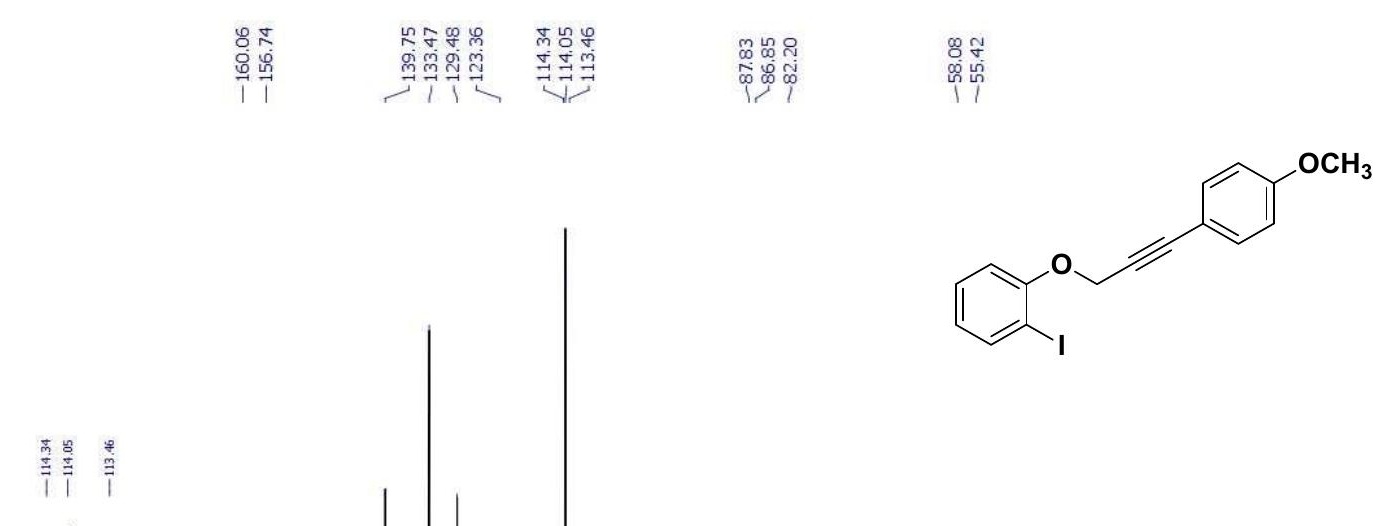


(75 $\left.\mathrm{MHz}, \mathrm{CDCl}_{3}\right)$

Figure S39. ${ }^{13} \mathrm{C}$ NMR of 1-iodo-2-((3-(4-methoxyphenyl)prop-2-yn-1-yl)oxy)benzene (18a).

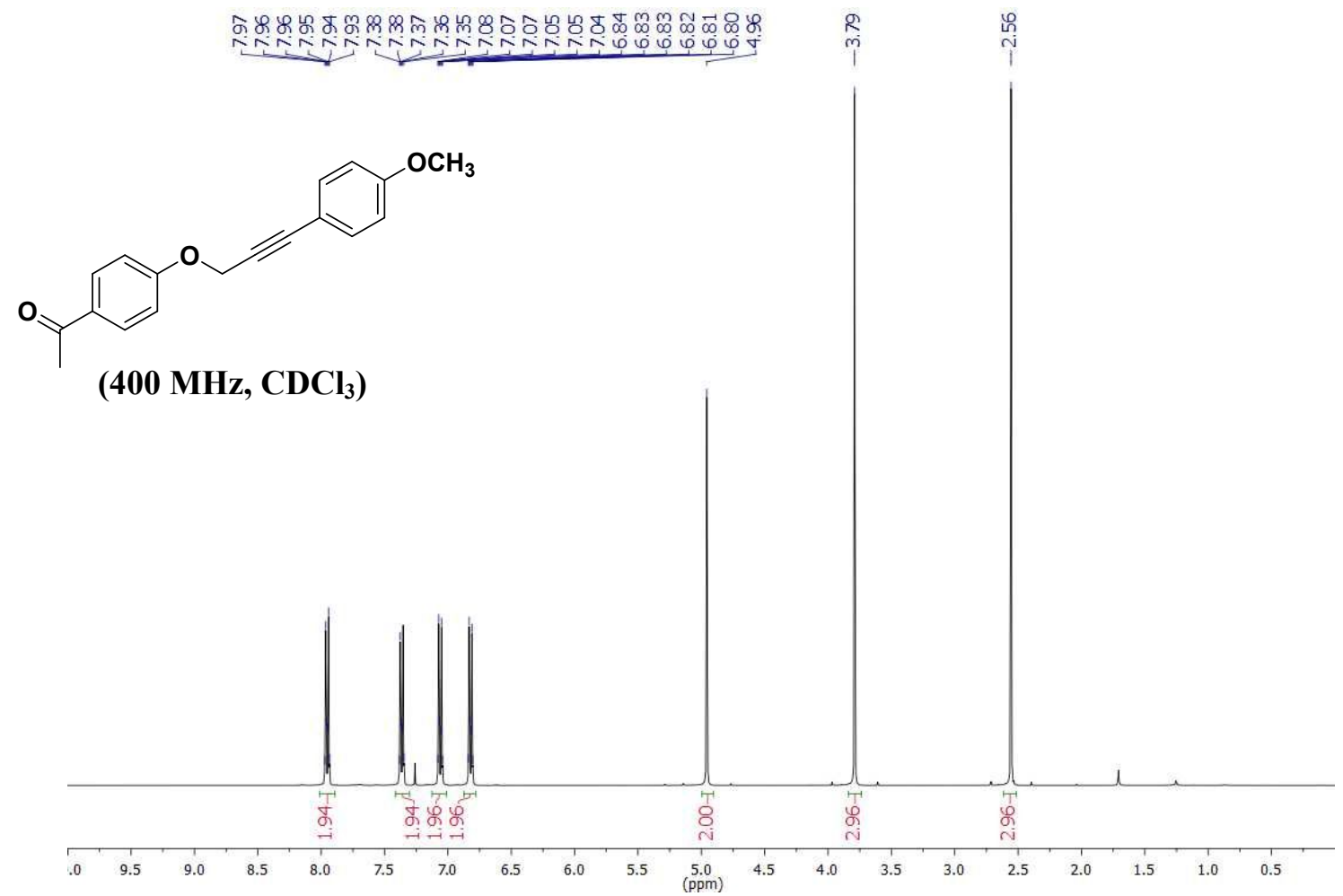

Figure S40. ${ }^{1} \mathrm{H}$ NMR of 1-(4-((3-(4-Methoxyphenyl)prop-2-yn-1-yl)oxy)phenyl)ethan-1-one (19a).

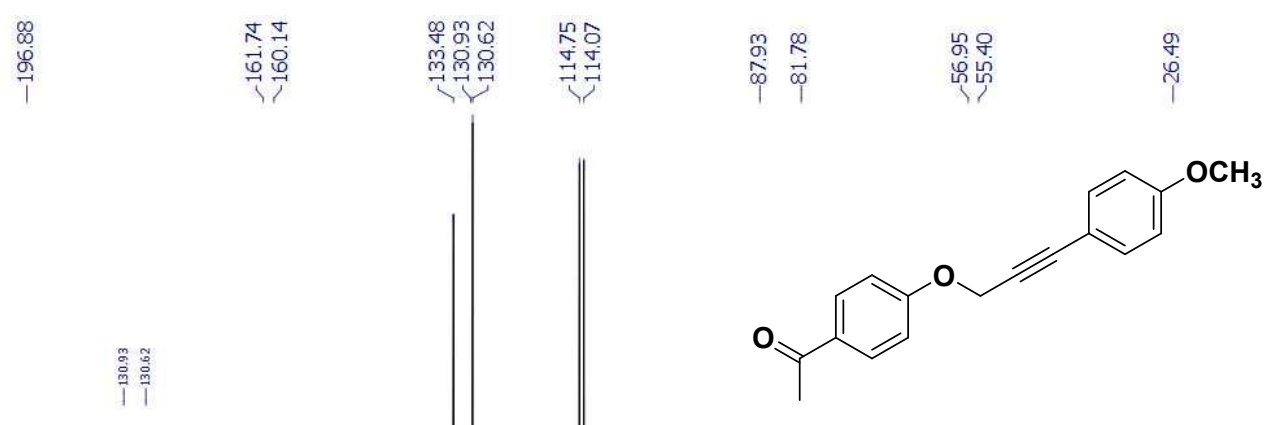


$\left(100 \mathrm{MHz}, \mathrm{CDCl}_{3}\right)$

Figure S41. ${ }^{13} \mathrm{C}$ NMR of 1-(4-((3-(4-Methoxyphenyl)prop-2-yn-1-yl)oxy)phenyl)ethan-1-one (19a).

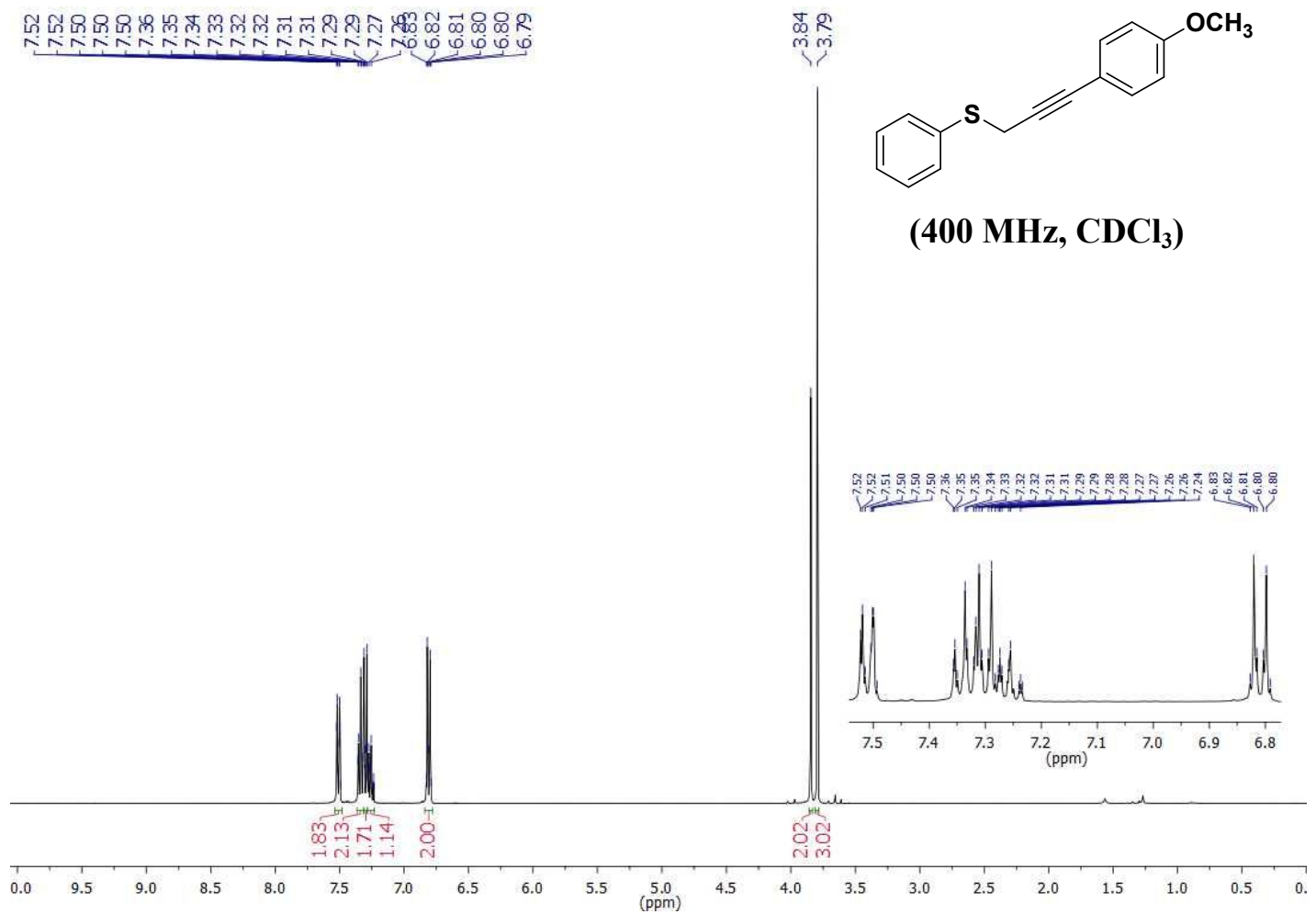

Figure S42. ${ }^{1} \mathrm{H}$ NMR of (3-(4-methoxyphenyl)prop-2-yn-1-yl)(phenyl)sulfane (20a).

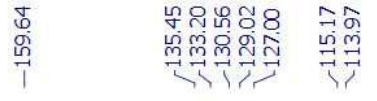

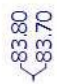

일

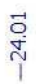<smiles>COc1ccc(C#CCSc2ccccc2)cc1</smiles> 
$\left(100 \mathrm{MHz}, \mathrm{CDCl}_{3}\right)$

Figure S43. ${ }^{13} \mathrm{C}$ NMR of (3-(4-methoxyphenyl)prop-2-yn-1-yl)(phenyl)sulfane (20a).

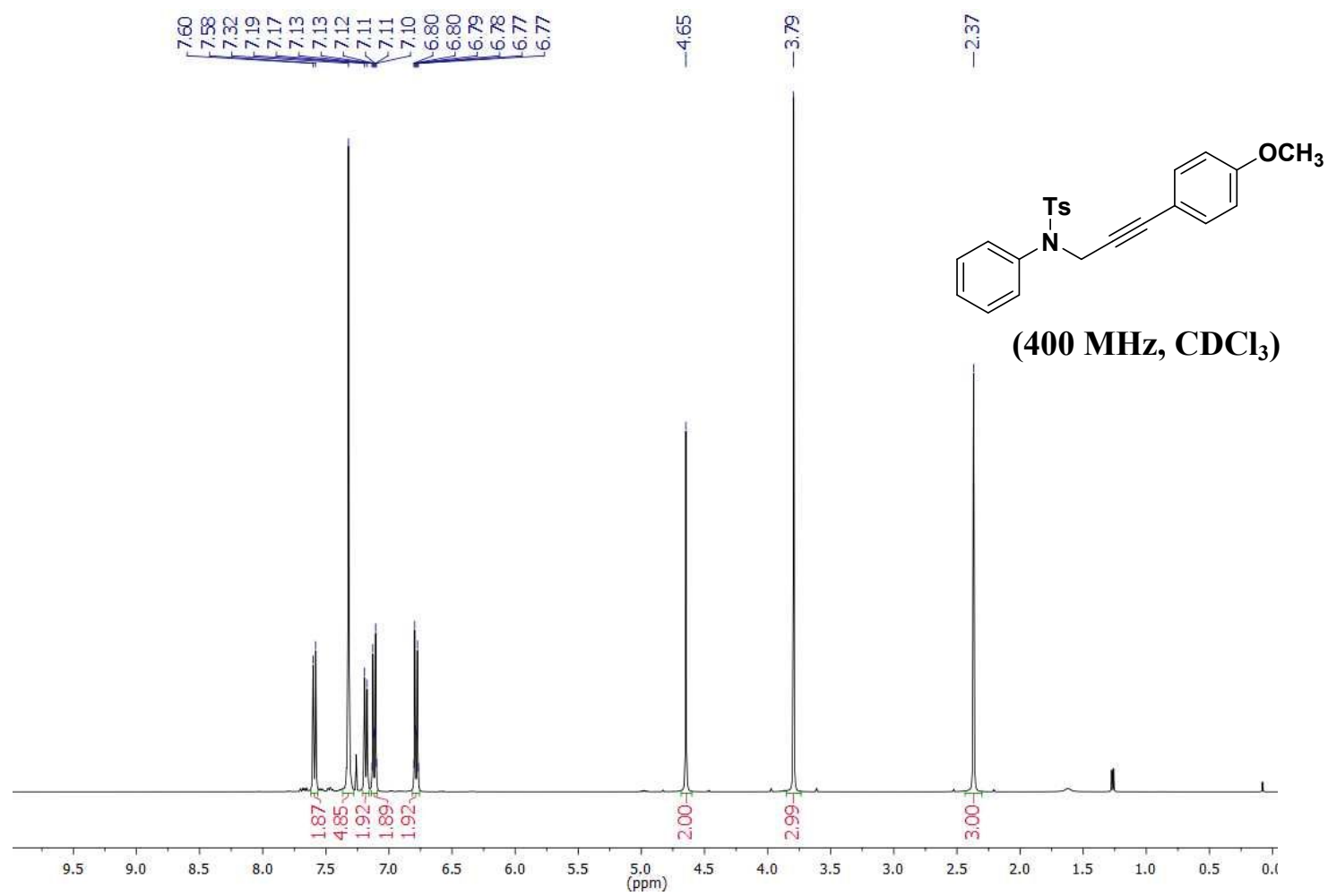

Figure S44. ${ }^{1} \mathrm{H}$ NMR of $N$-(3-(4-methoxyphenyl)prop-2-yn-1-yl)-4-methyl- $N$ phenylbenzenesulfonamide (21a).

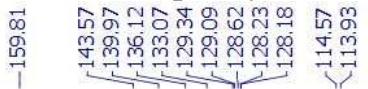

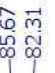

กัช

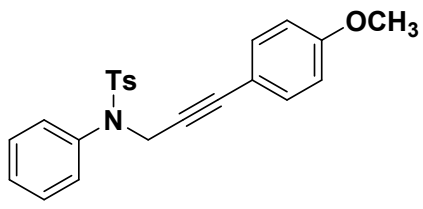

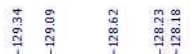


(100 MHz, $\left.\mathrm{CDCl}_{3}\right)$

Figure S45. ${ }^{13} \mathrm{C}$ NMR of $N$-(3-(4-methoxyphenyl)prop-2-yn-1-yl)-4-methyl- $N$ phenylbenzenesulfonamide (21a).

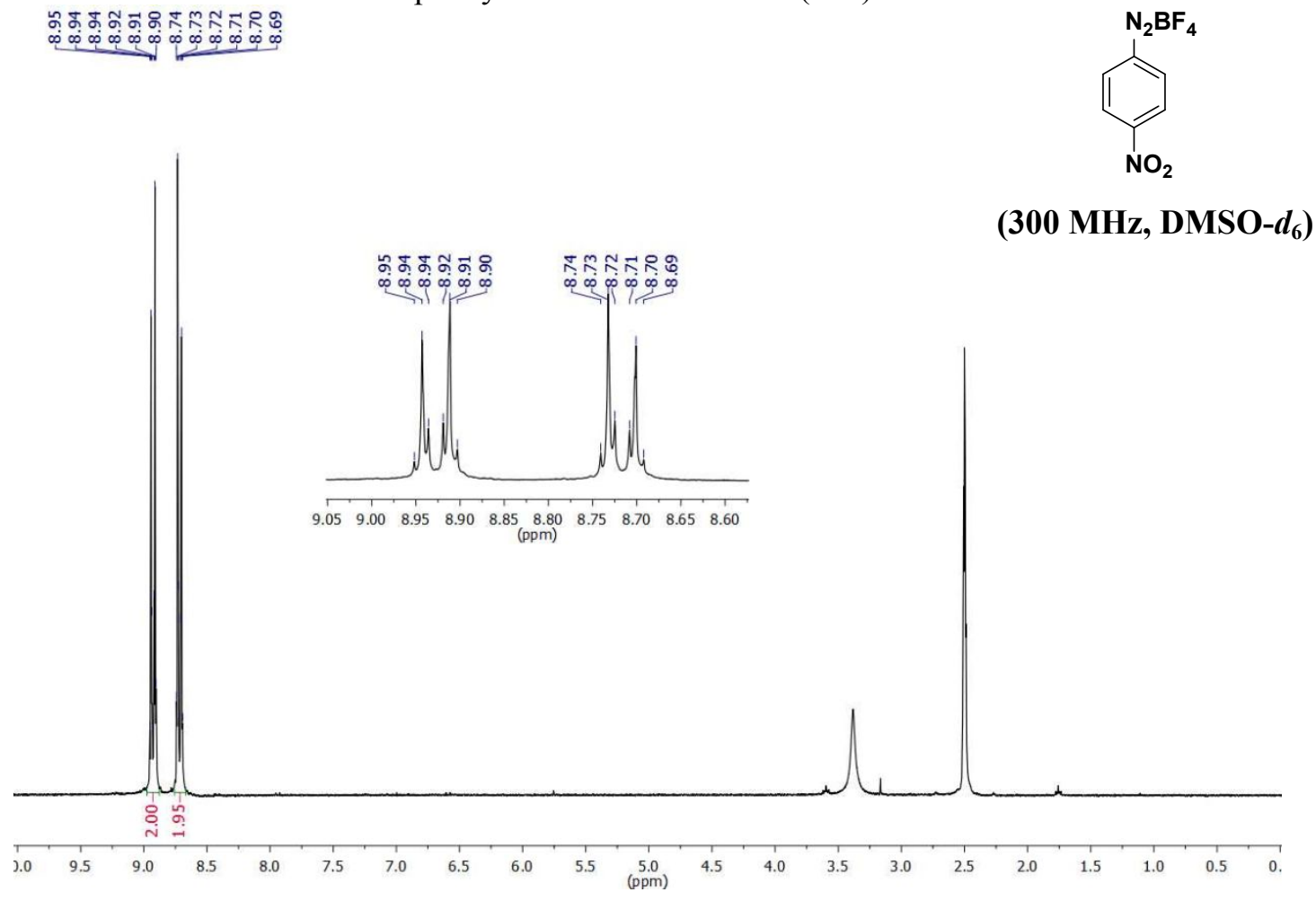

Figure S46. ${ }^{1} \mathrm{H}$ NMR of 4-nitrobenzenediazonium tetrafluoroborate (1b).

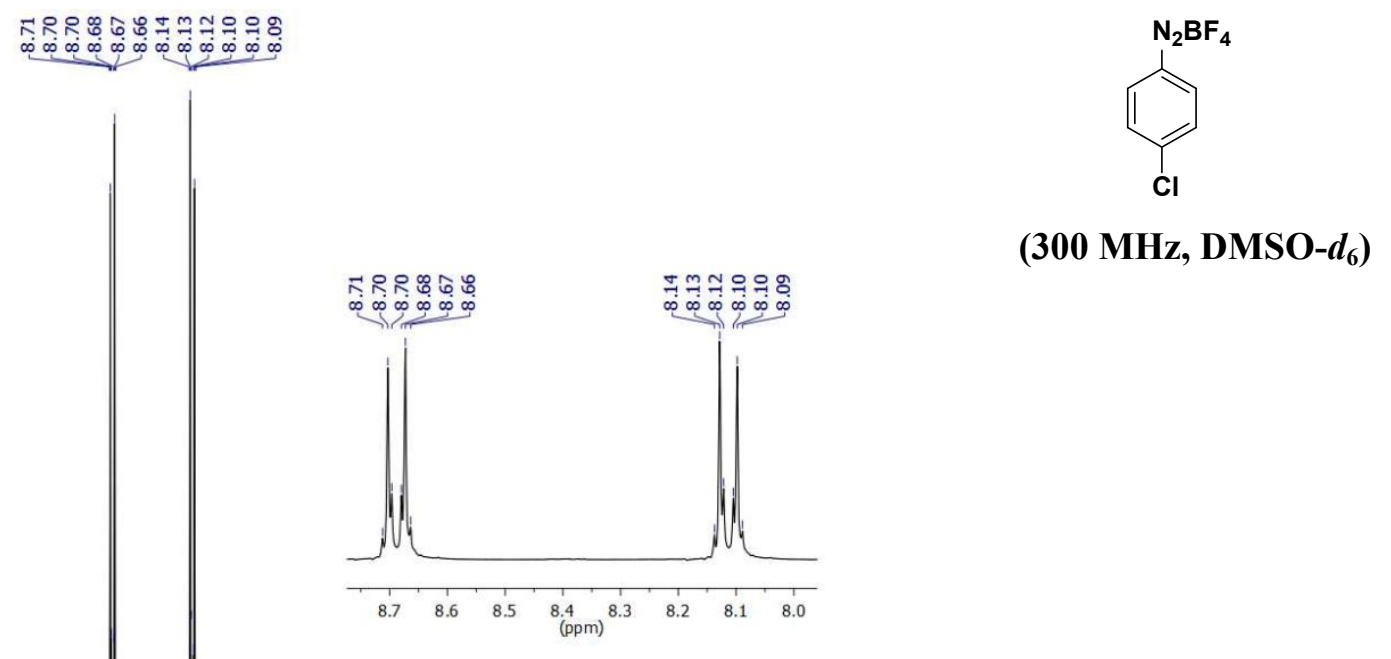


Figure S47. ${ }^{1} \mathrm{H}$ NMR of 4-chlorobenzenediazonium tetrafluoroborate (2b).

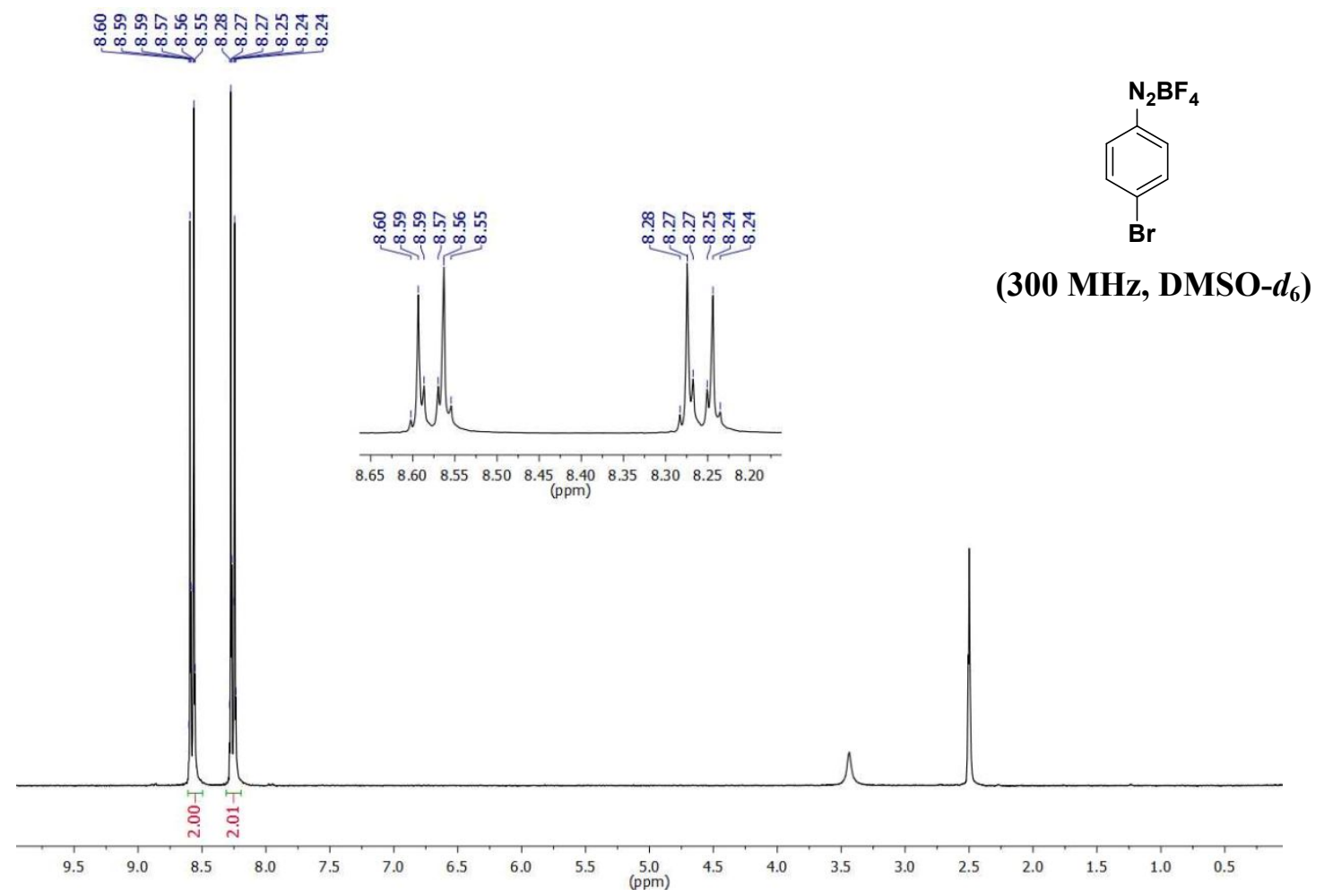

Figure S48. ${ }^{1} \mathrm{H}$ NMR of 4-bromobenzenediazonium tetrafluoroborate (3b).

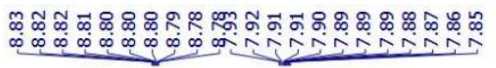

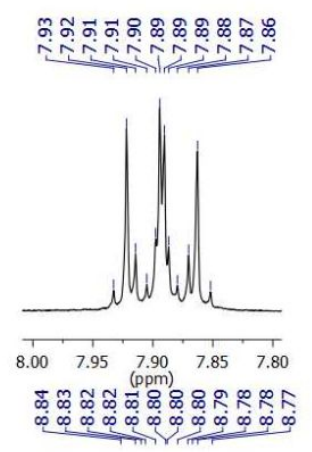

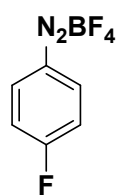

(300 MHz, DMSO- $d_{6}$ ) 
Figure S49. ${ }^{1} \mathrm{H}$ NMR of 4-fluorobenzenediazonium tetrafluoroborate (4b).

$\underset{\substack{\infty \\ \infty}}{\infty} \prod_{\substack{\infty \\ \infty}}^{\infty}$

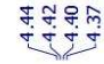

$\mathrm{N}_{2} \mathrm{BF}_{4}$

$\overbrace{0}^{1}$

(300 MHz, DMSO-d )

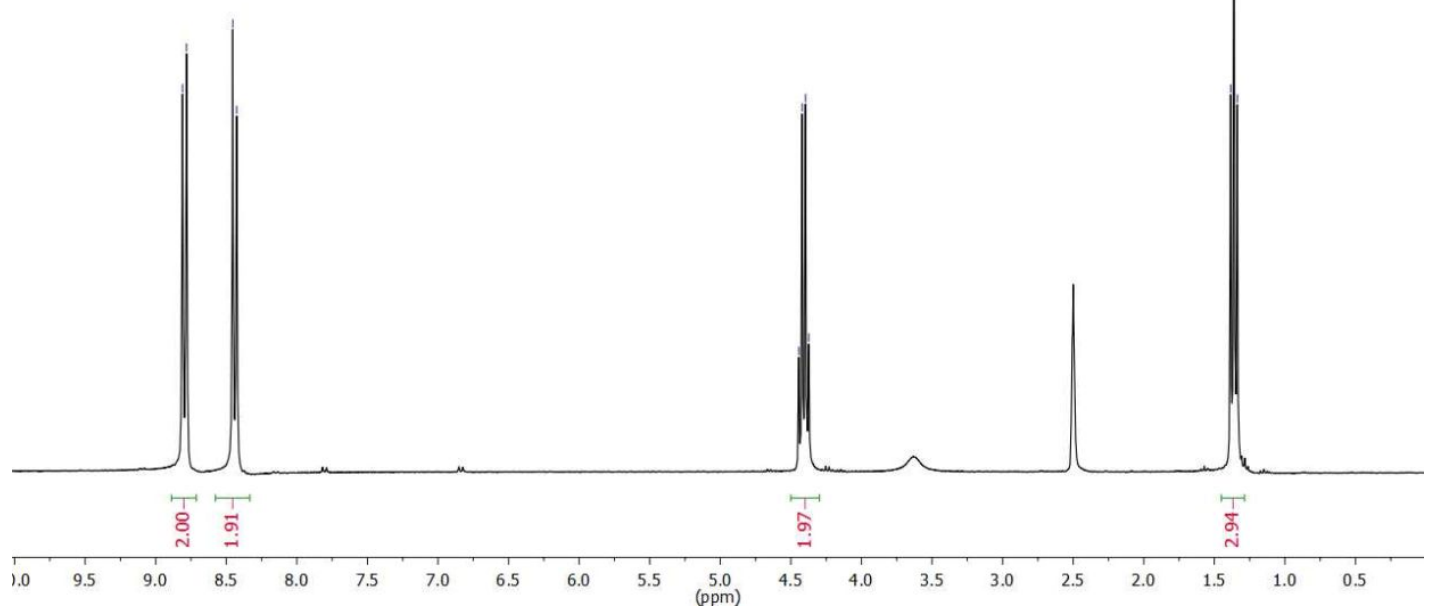

Figure S50. ${ }^{1} \mathrm{H}$ NMR of 4-ethoxycarbonylbenzenediazonium tetrafluoroborate (5b).
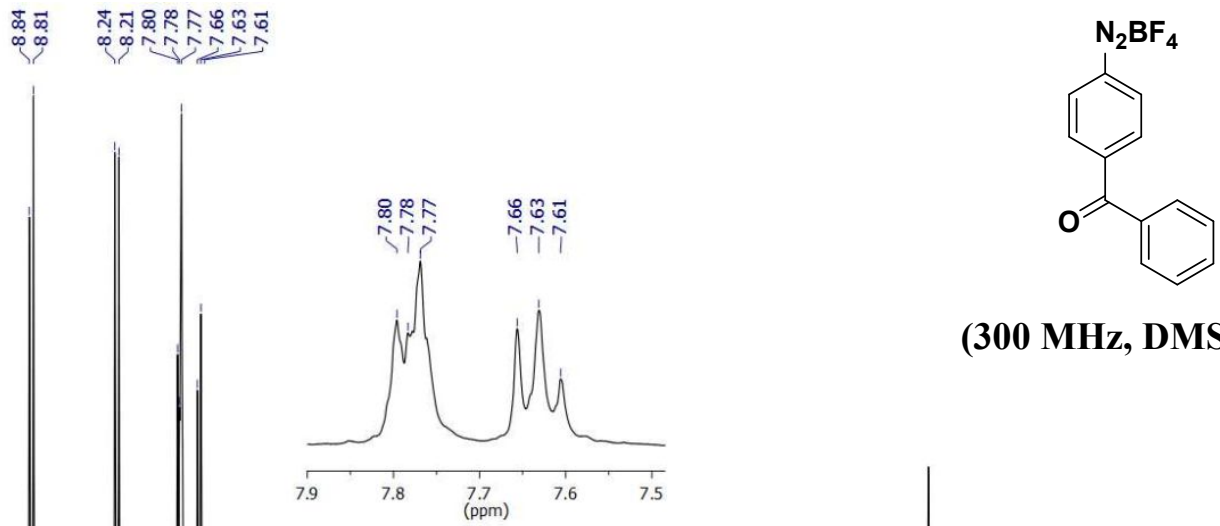

(300 MHz, DMSO- $\left.d_{6}\right)$ 
Figure S51. ${ }^{1} \mathrm{H}$ NMR of 4-benzoylbenzenediazonium tetrafluoroborate (6b).

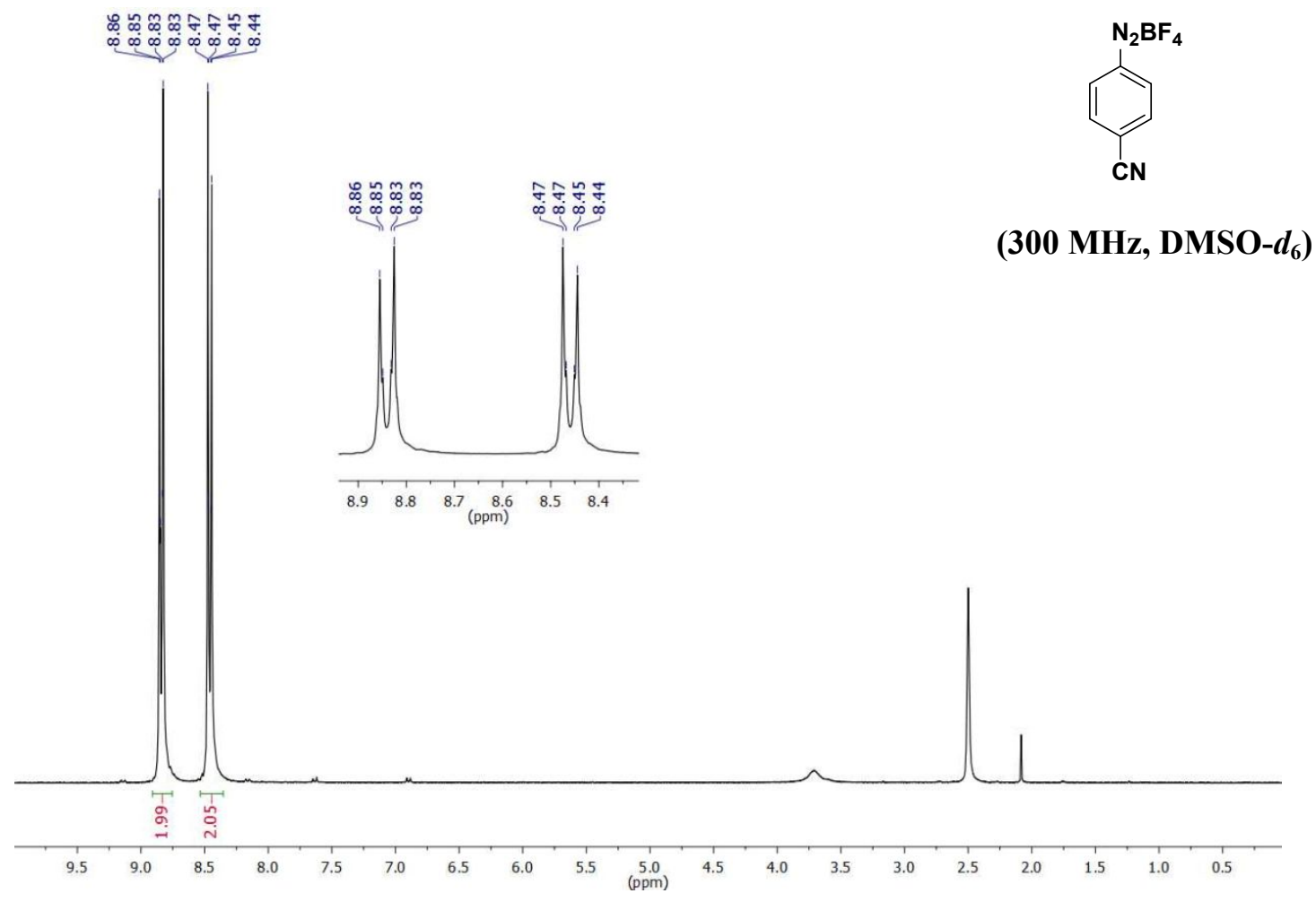

Figure S52. ${ }^{1} \mathrm{H}$ NMR of 4-cyanobenzenediazonium tetrafluoroborate (7b).

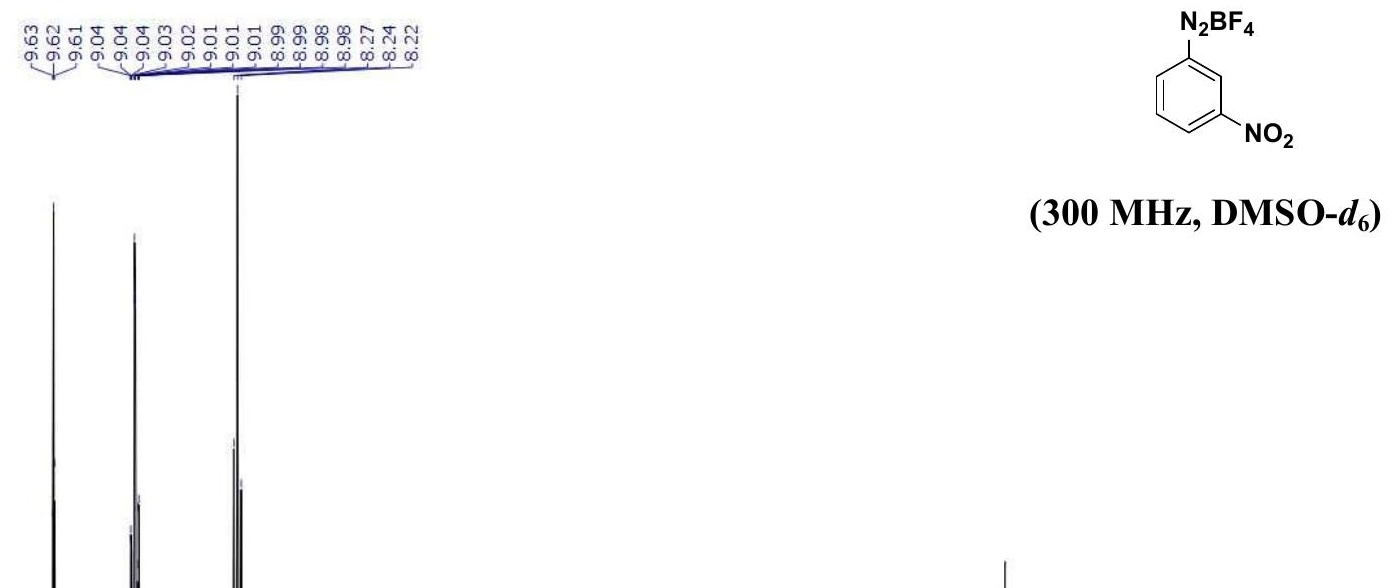


Figure S53. ${ }^{1} \mathrm{H}$ NMMR of 3-nitrobenzenediazonium tetrafluoroborate (8b). तु

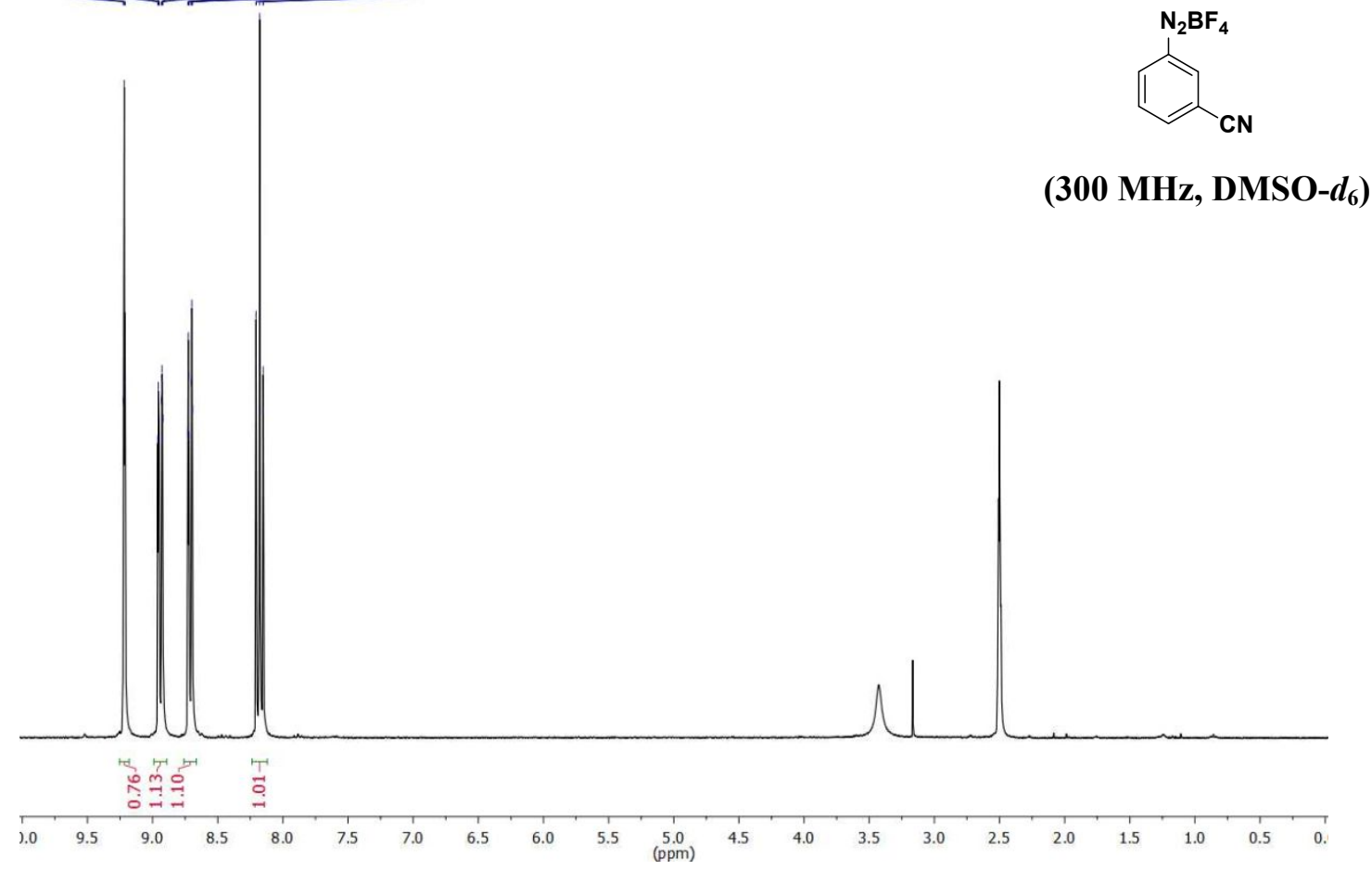

Figure S54. ${ }^{1} \mathrm{H}$ NMR of 3-cyanobenzenediazonium tetrafluoroborate (9b). 


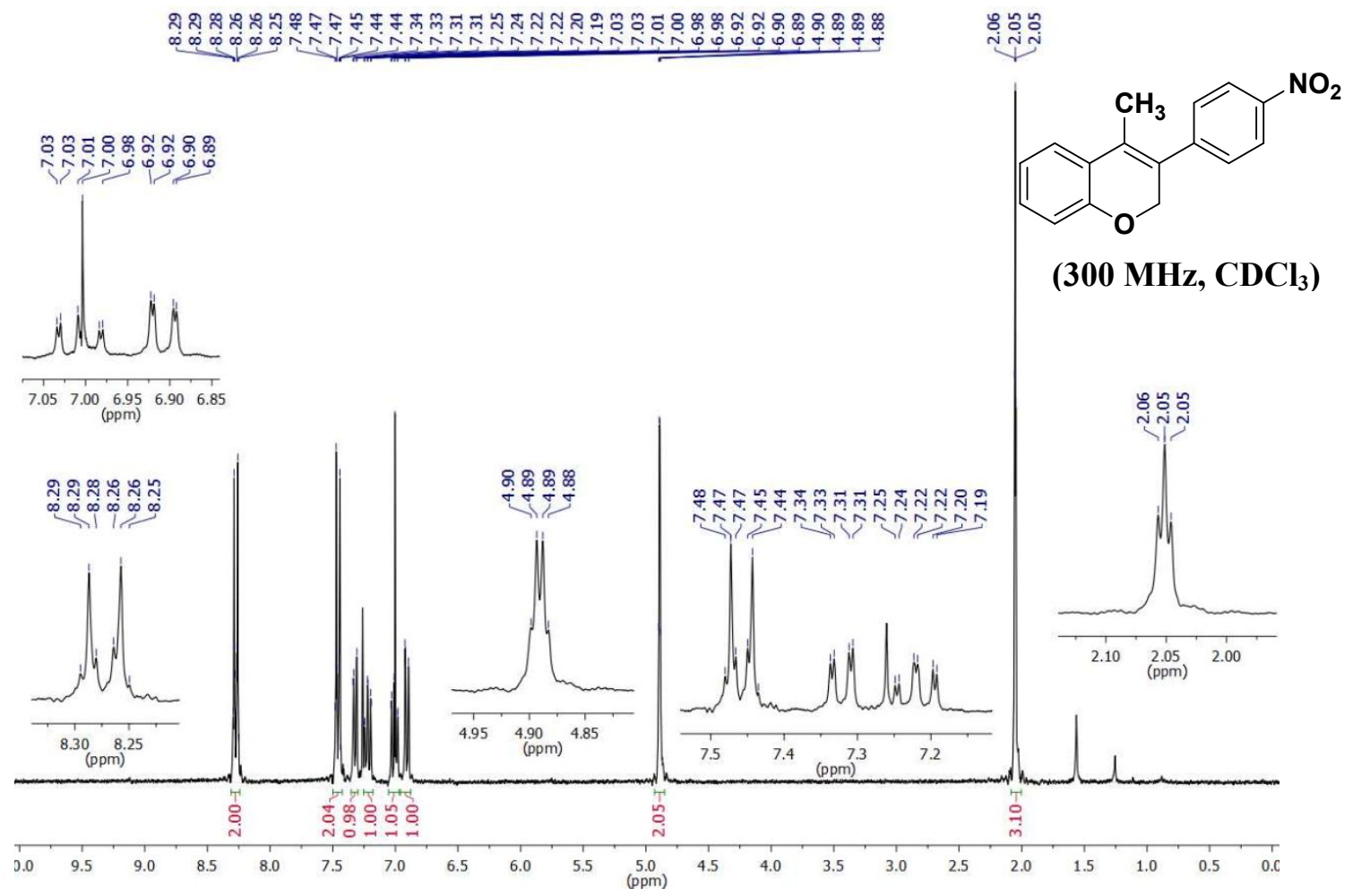

Figure S55. ${ }^{1} \mathrm{H}$ NMR of 4-methyl-3-(4-nitrophenyl)-2 $H$-chromene (1c).

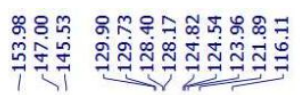<smiles>CC1=C(c2ccc([N+](=O)[O-])cc2)COc2ccccc21</smiles>

(75 MHz, $\mathrm{CDCl}_{3}$ )

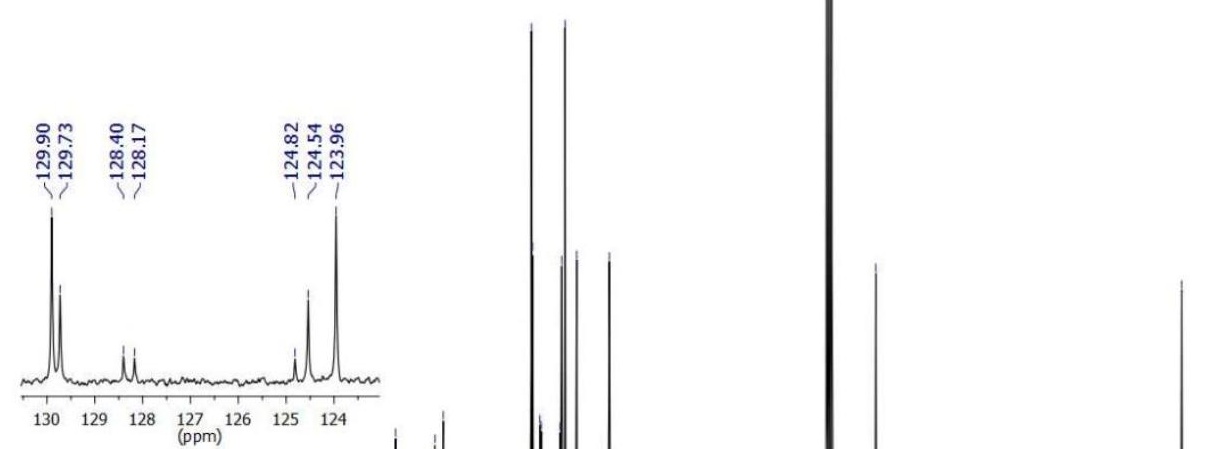


Figure S56. ${ }^{13} \mathrm{C}$ NMR of 4-methyl-3-(4-nitrophenyl)-2H-chromene (1c).

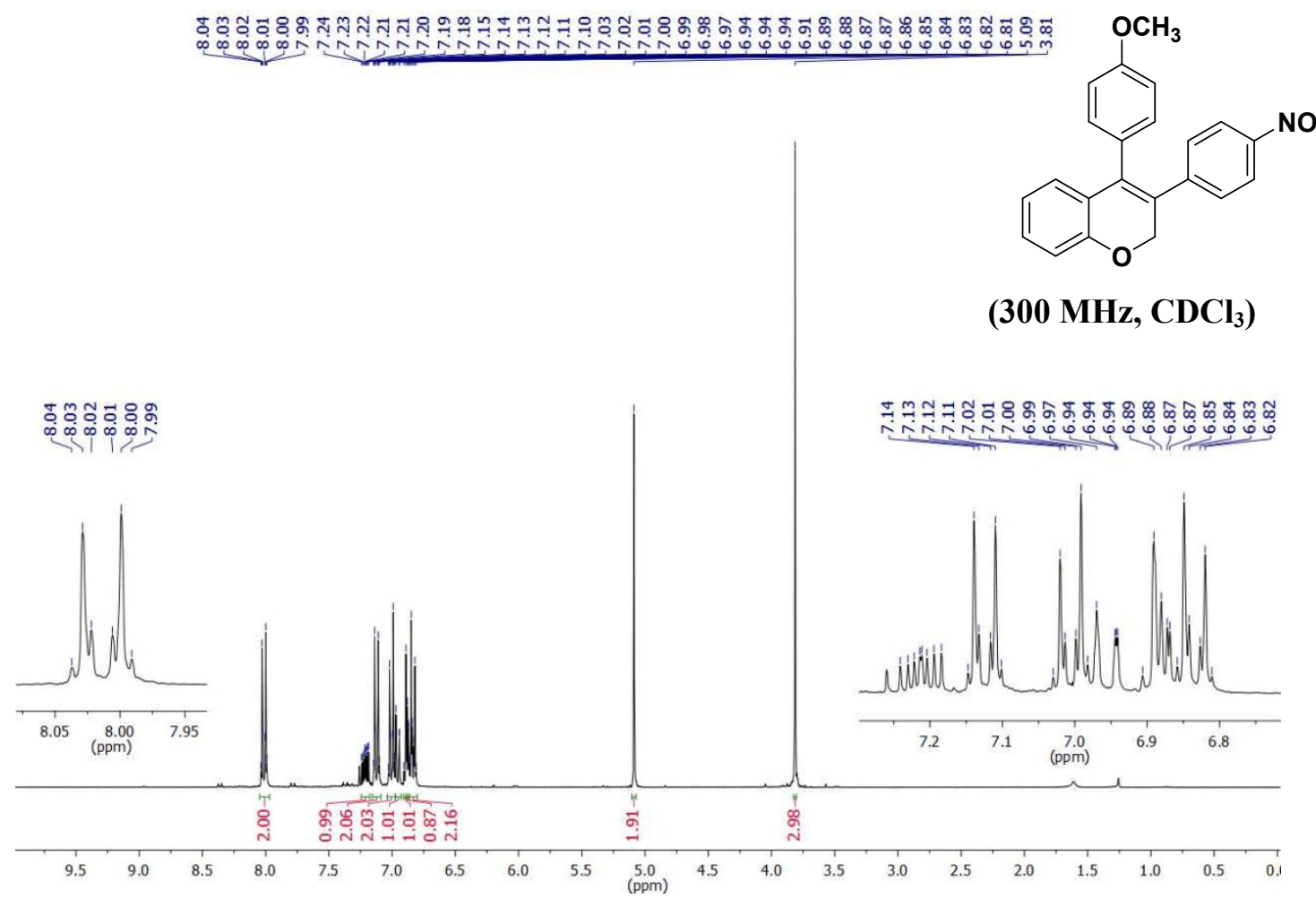

Figure S57. ${ }^{1} \mathrm{H}$ NMR of 4-(4-methoxyphenyl)-3-(4-nitrophenyl)-2H-chromene (2c).

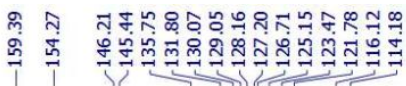

¿o

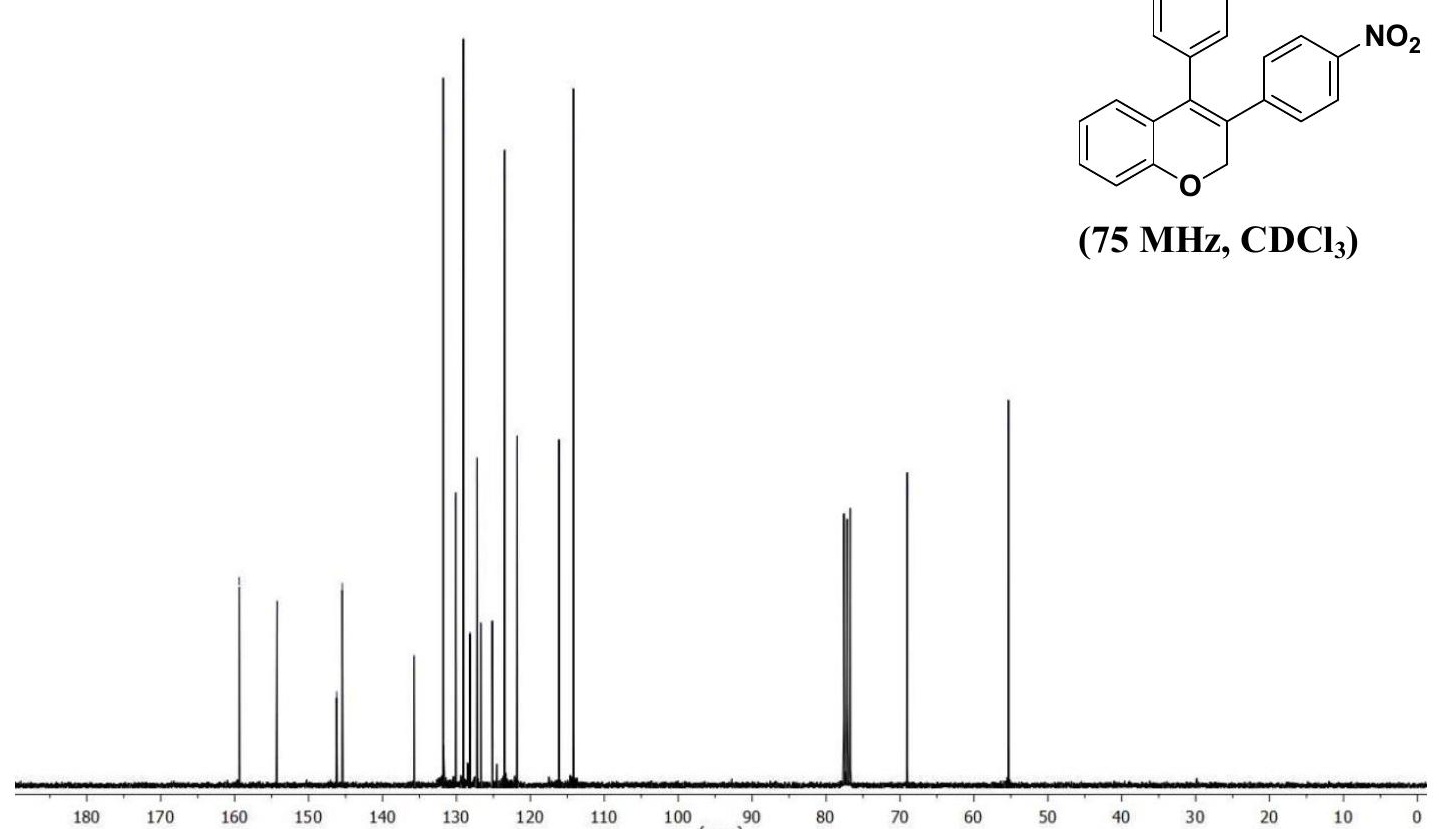


Figure S58. ${ }^{13} \mathrm{C}$ NMR of 4-(4-methoxyphenyl)-3-(4-nitrophenyl)-2H-chromene (2c).

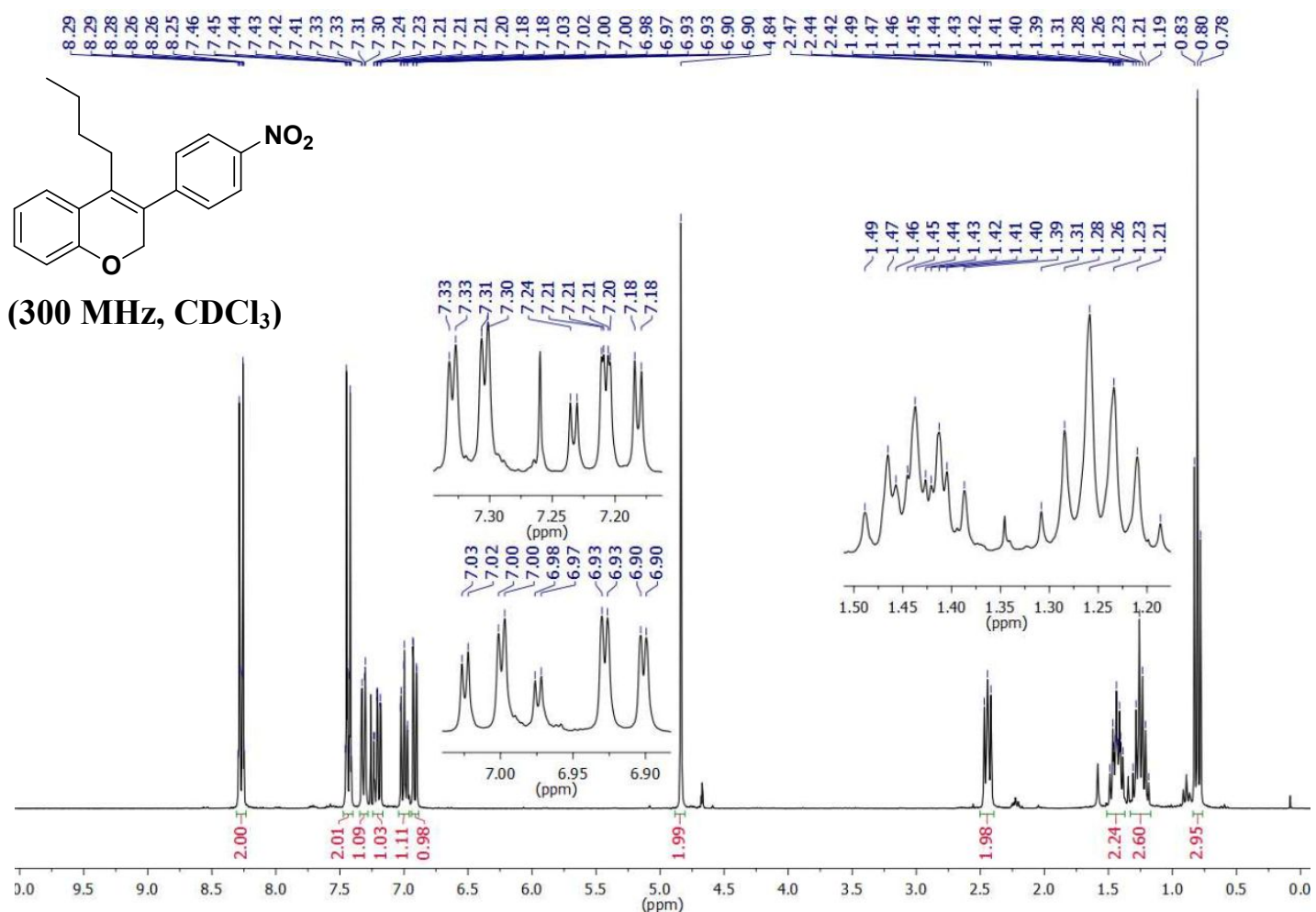

Figure S59. ${ }^{1} \mathrm{H}$ NMR of 4-butyl-3-(4-nitrophenyl)-2H-chromene (3c).
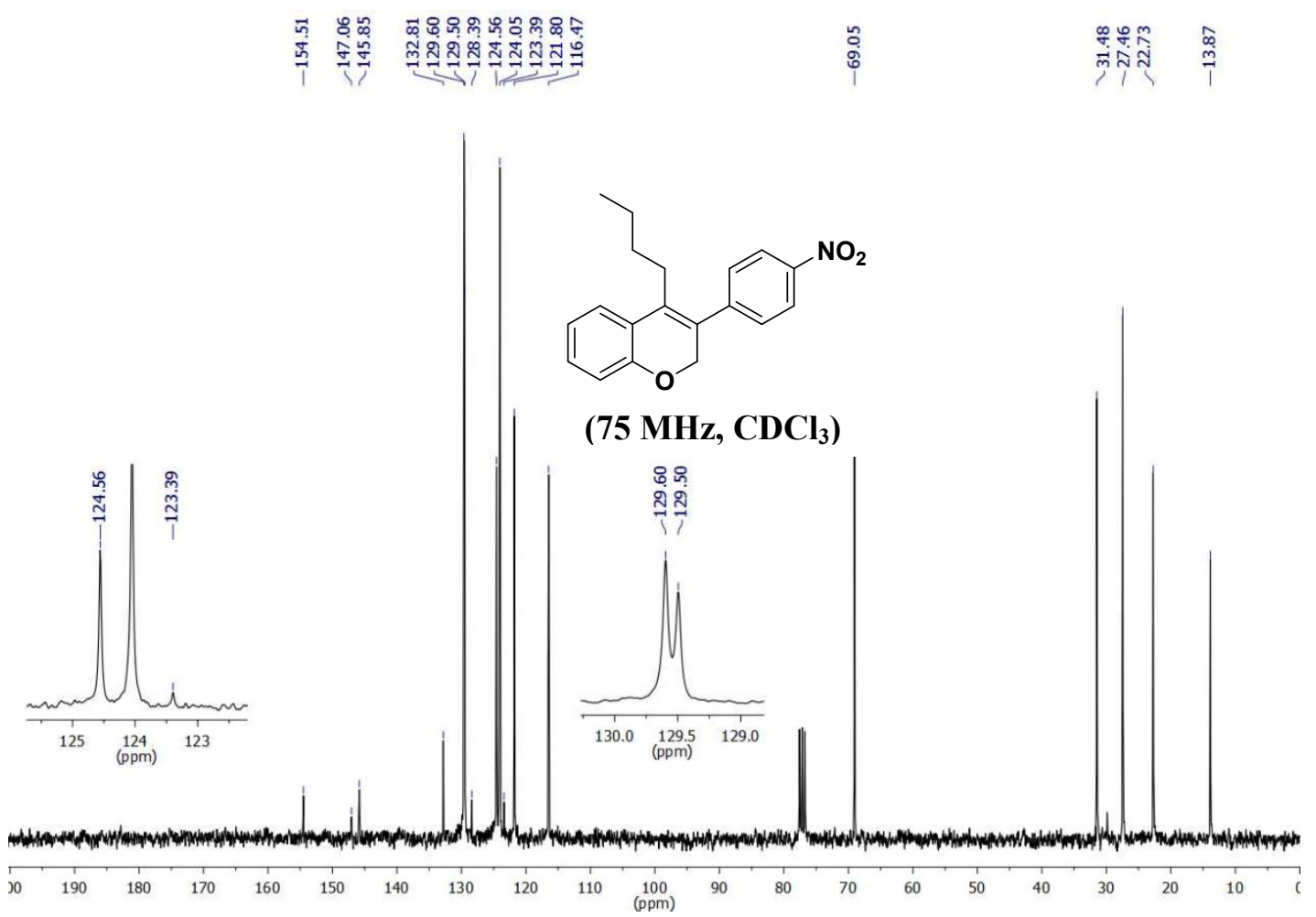
Figure S60. ${ }^{13} \mathrm{C}$ NMR of 4-butyl-3-(4-nitrophenyl)-2H-chromene (3c).

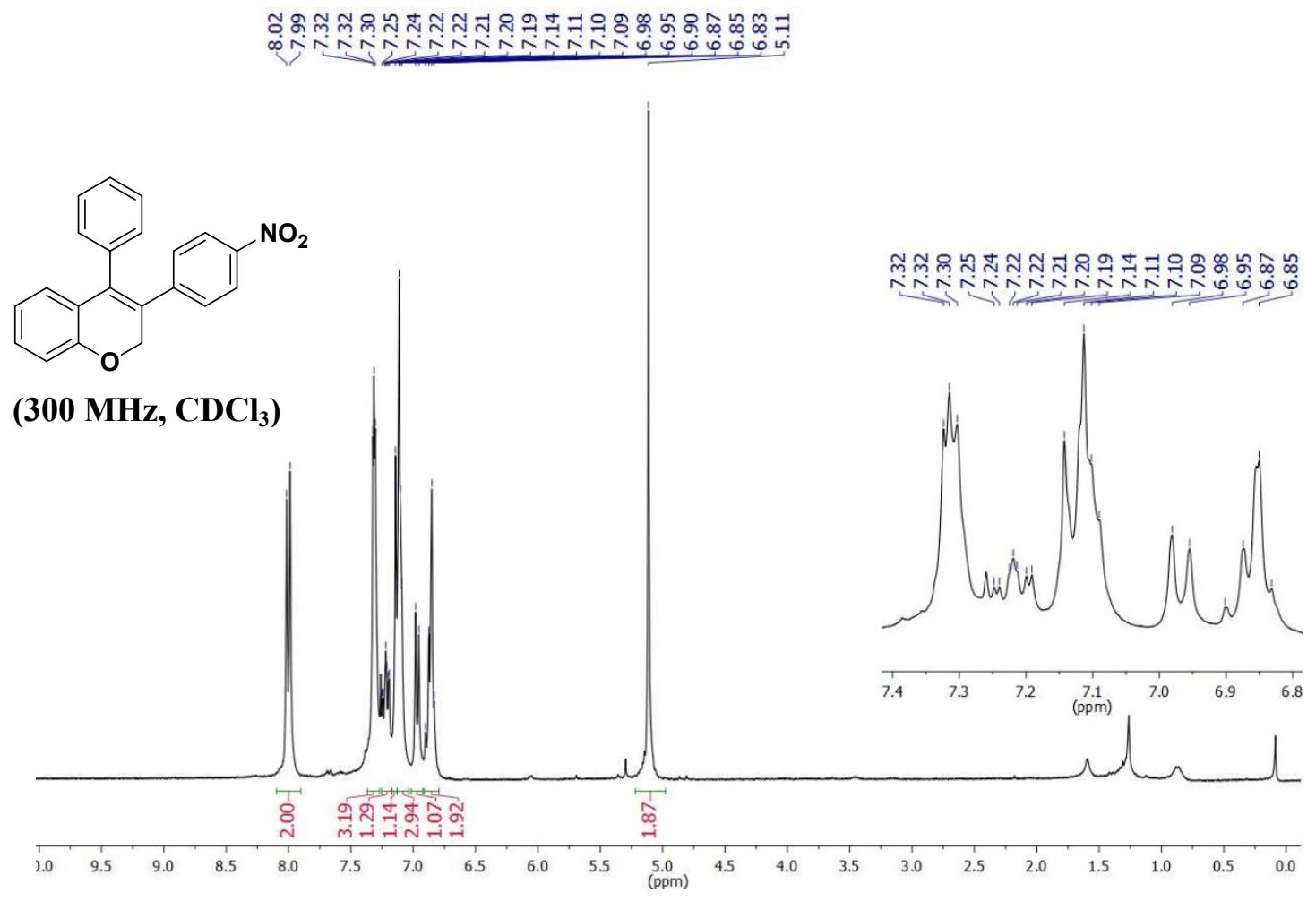

Figure S61. ${ }^{1} \mathrm{H}$ NMR of 3-(4-nitrophenyl)-4-phenyl-2H-chromene (4c).

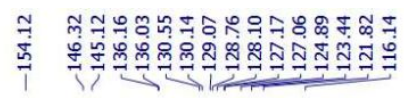

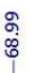<smiles>O=[N+]([O-])c1ccc(C2=C(c3ccccc3)c3ccccc3OC2)cc1</smiles>

(75 MHz, $\left.\mathrm{CDCl}_{3}\right)$

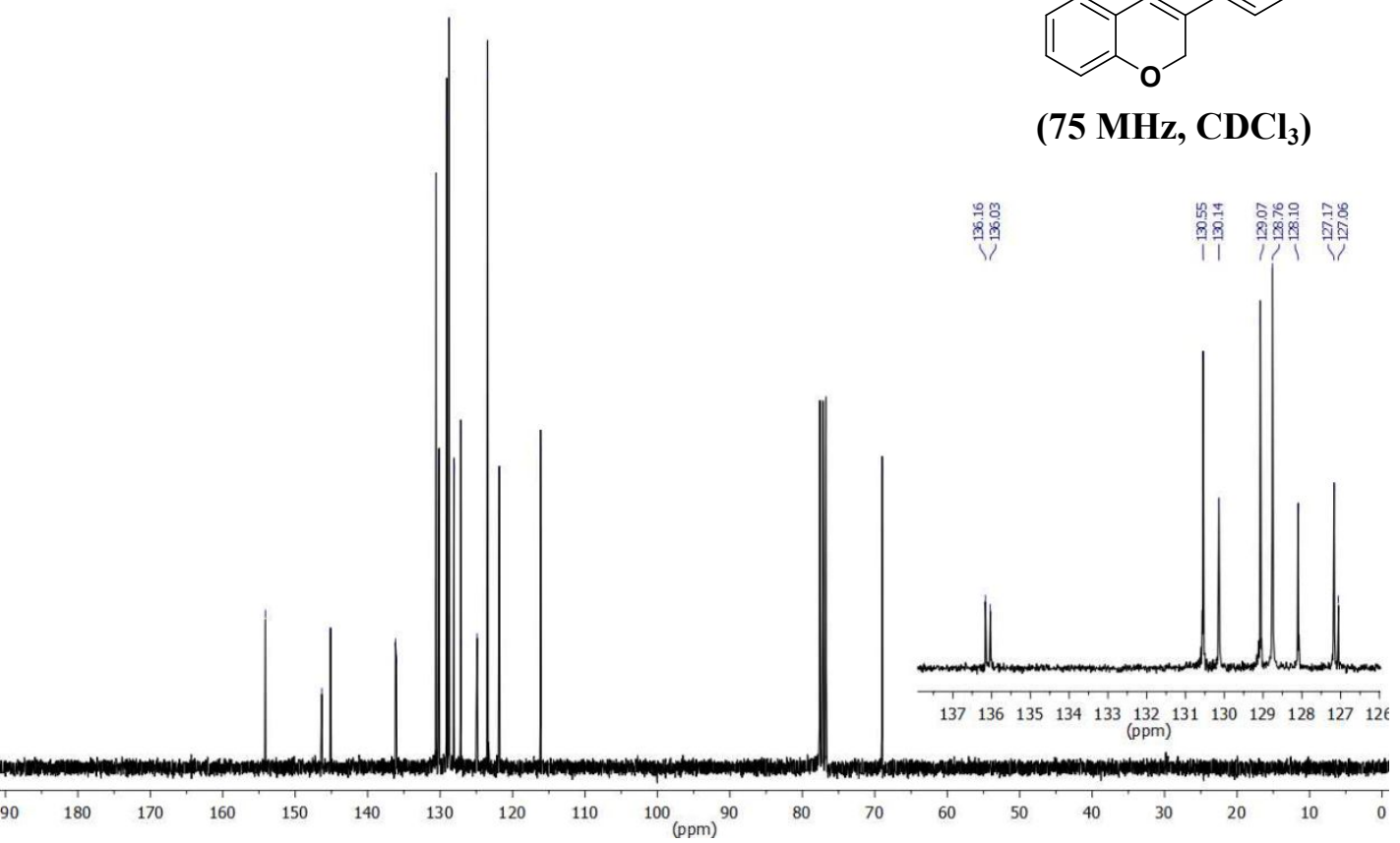


Figure S62. ${ }^{13} \mathrm{C}$ NMR of 3-(4-nitrophenyl)-4-phenyl-2H-chromene (4c).

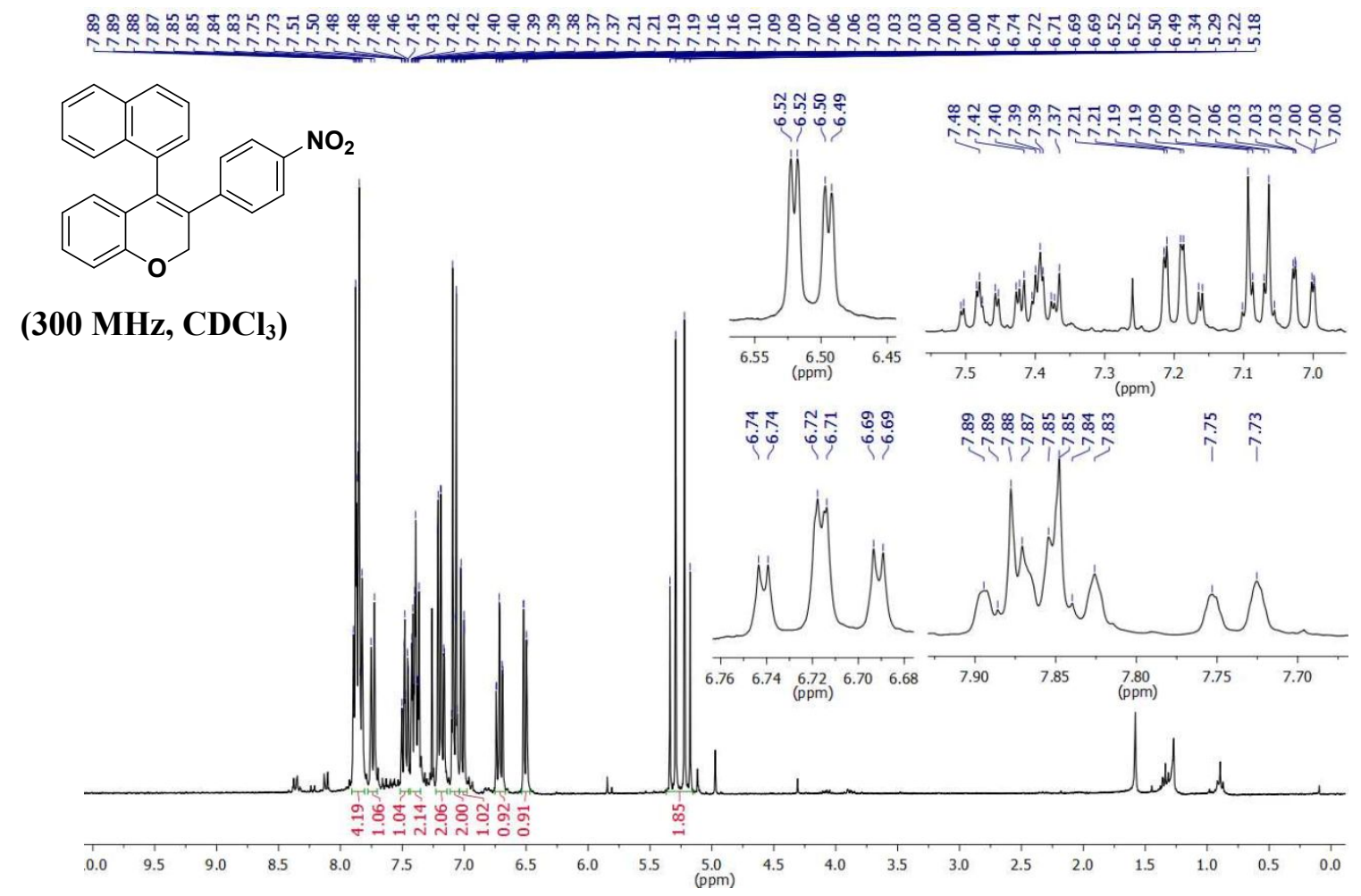

Figure S63. ${ }^{1} \mathrm{H}$ NMR of 4-(naphthalen-1-yl)-3-(4-nitrophenyl)-2H-chromene (5c).

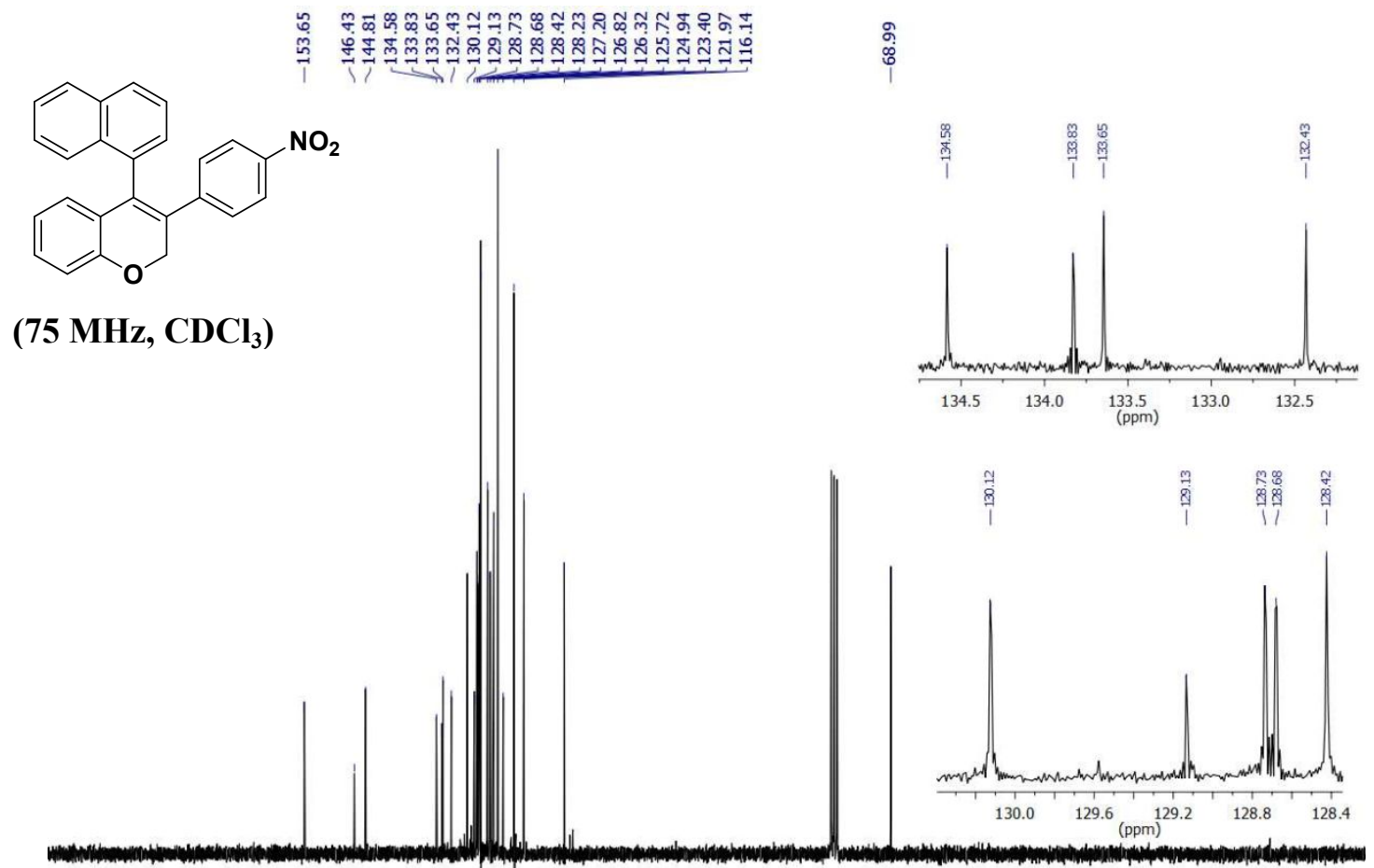


Figure S64. ${ }^{13} \mathrm{C}$ NMR of 4-(naphthalen-1-yl)-3-(4-nitrophenyl)-2 $H$-chromene (5c).

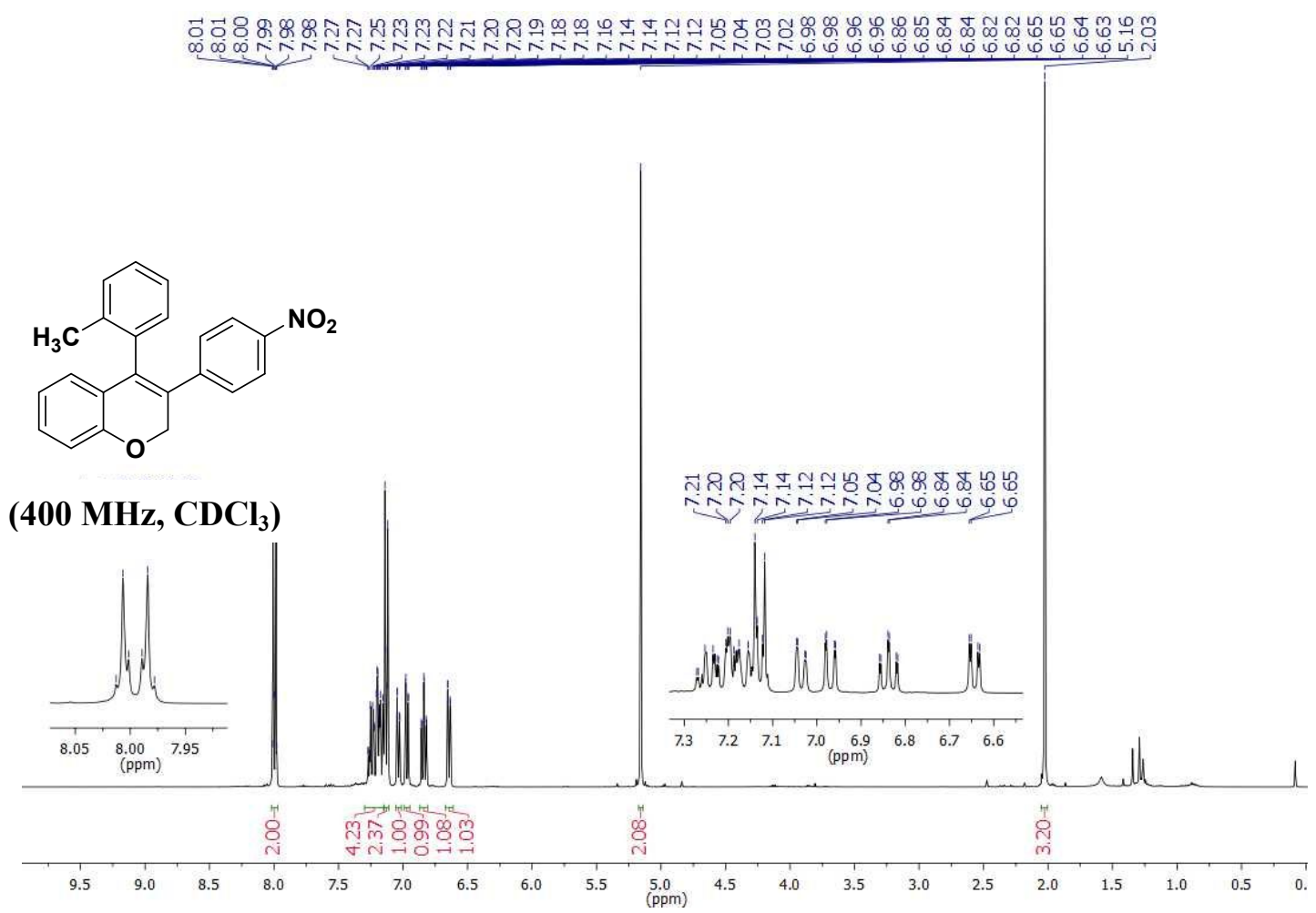

Figure S65. ${ }^{1} \mathrm{H}$ NMR of 3-(4-Nitrophenyl)-4-(o-tolyl)-2H-chromene (6c).

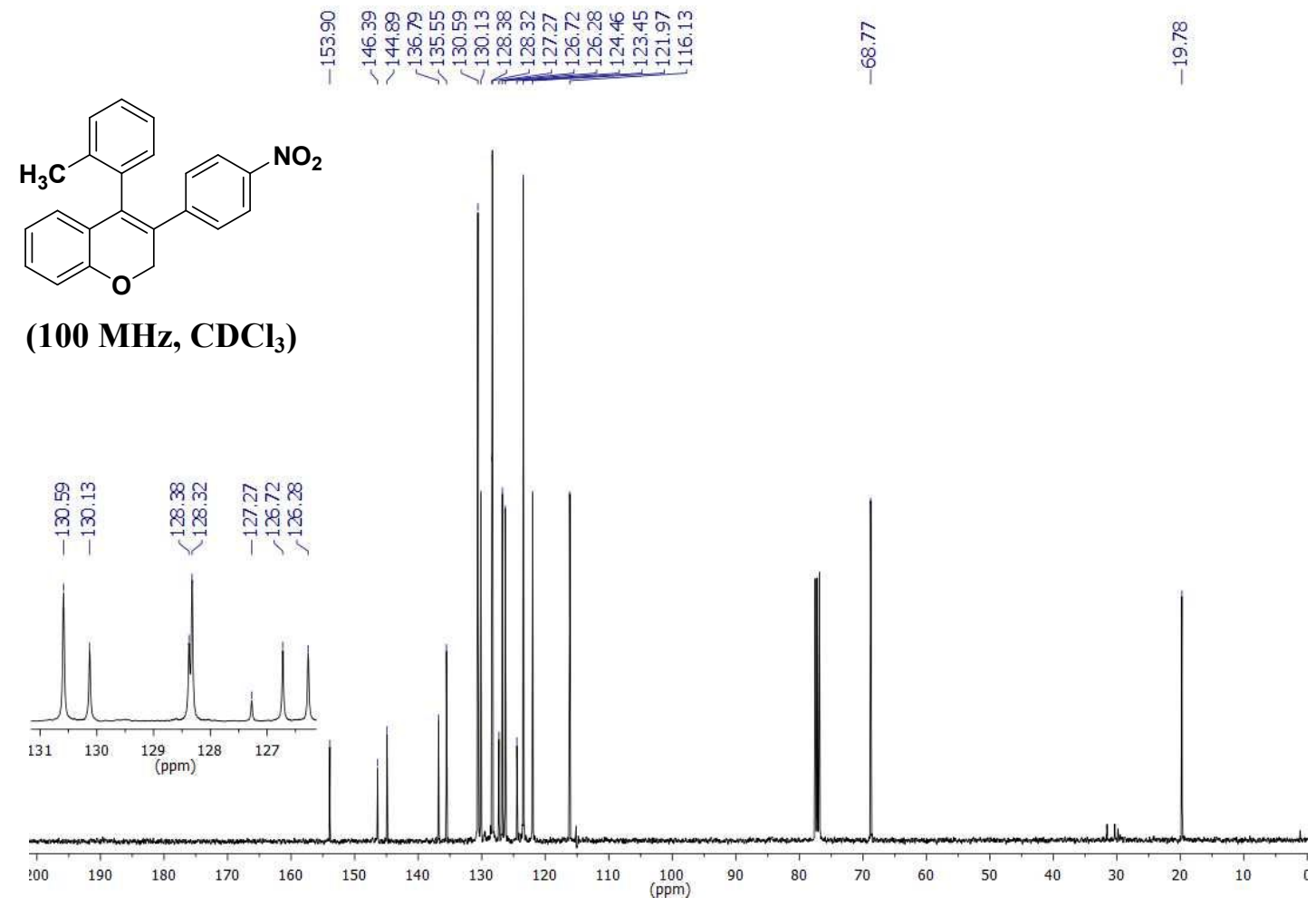


Figure S66. ${ }^{1} \mathrm{H}$ NMR of 3-(4-Nitrophenyl)-4-(o-tolyl)-2H-chromene (6c).

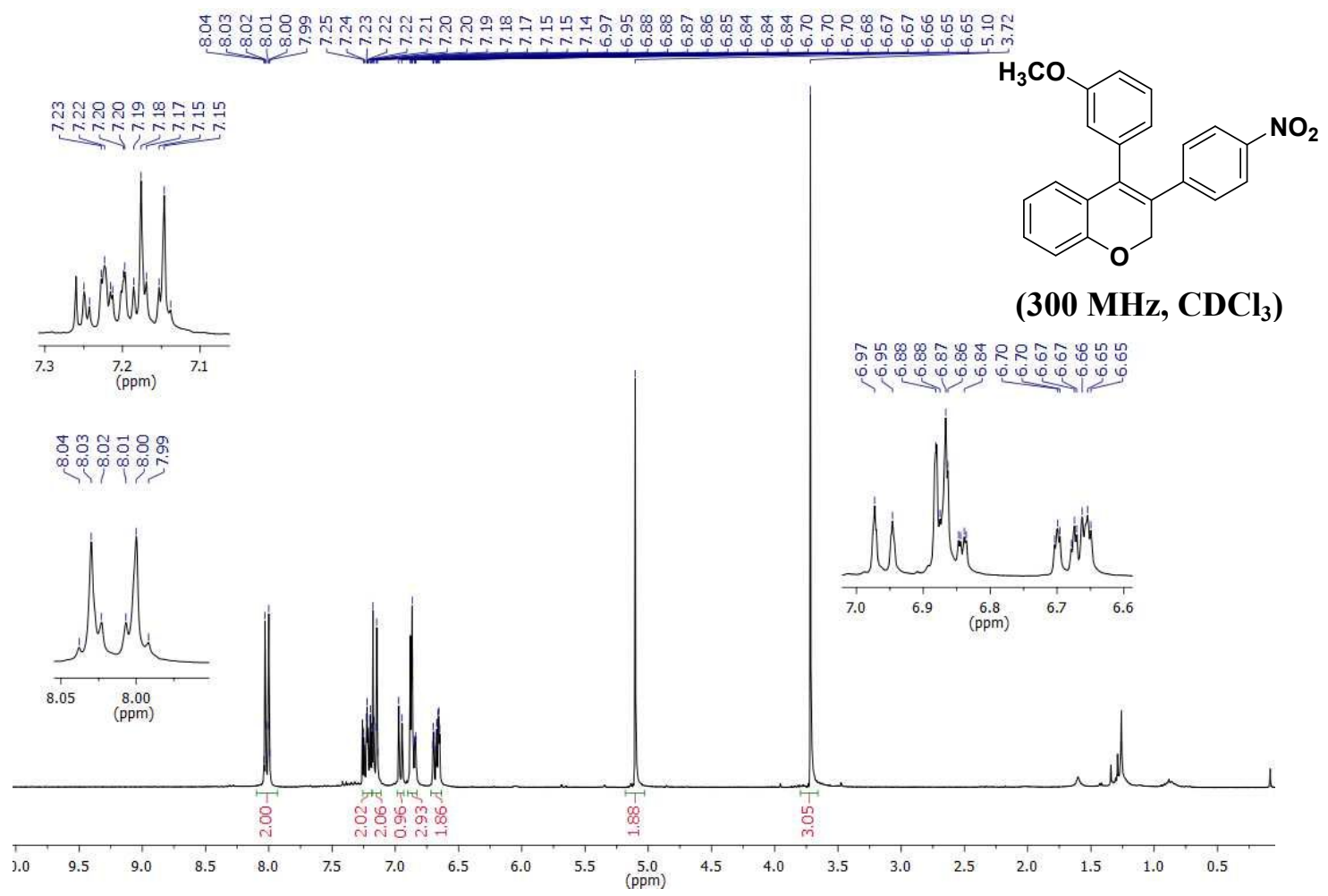

Figure S67. ${ }^{1} \mathrm{H}$ NMR of 4-(3-methoxyphenyl)-3-(4-nitrophenyl)-2H-chromene (7c).
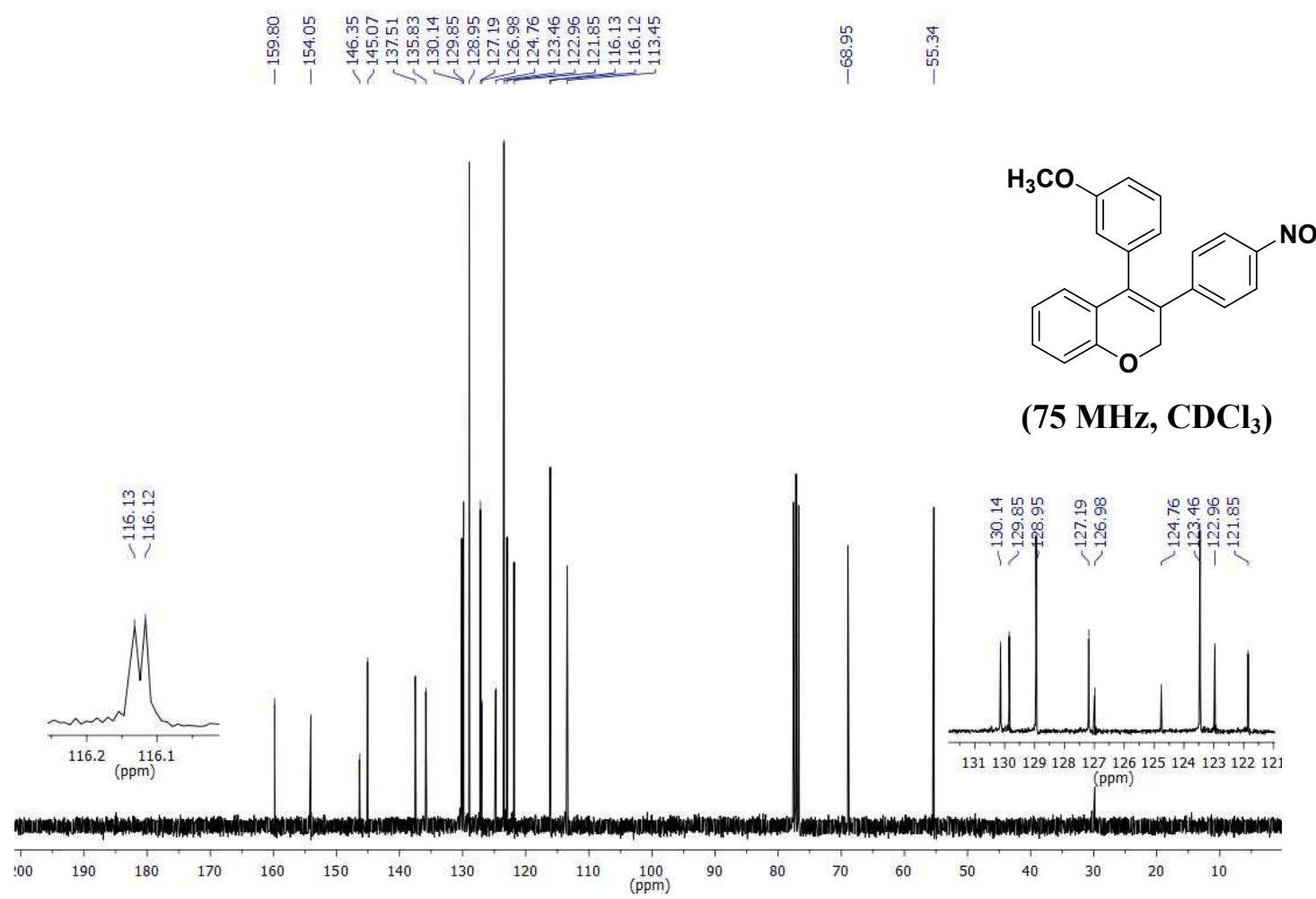
Figure S68. ${ }^{13} \mathrm{C}$ NMR of 4-(3-methoxyphenyl)-3-(4-nitrophenyl)-2H-chromene (7c).

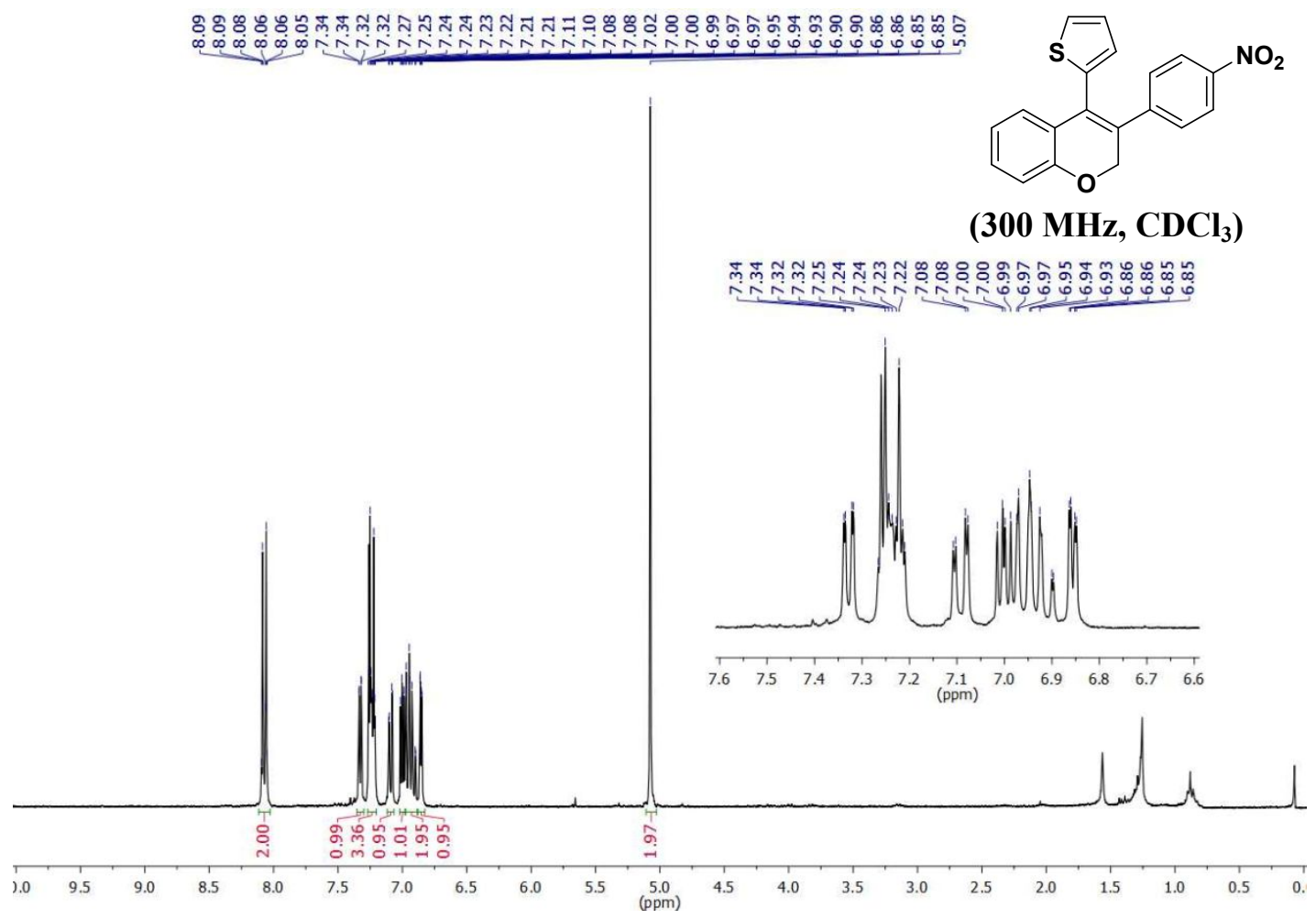

Figure S69. ${ }^{1} \mathrm{H}$ NMR of 3-(4-nitrophenyl)-4-(thiophen-2-yl)-2H-chromene (9c).
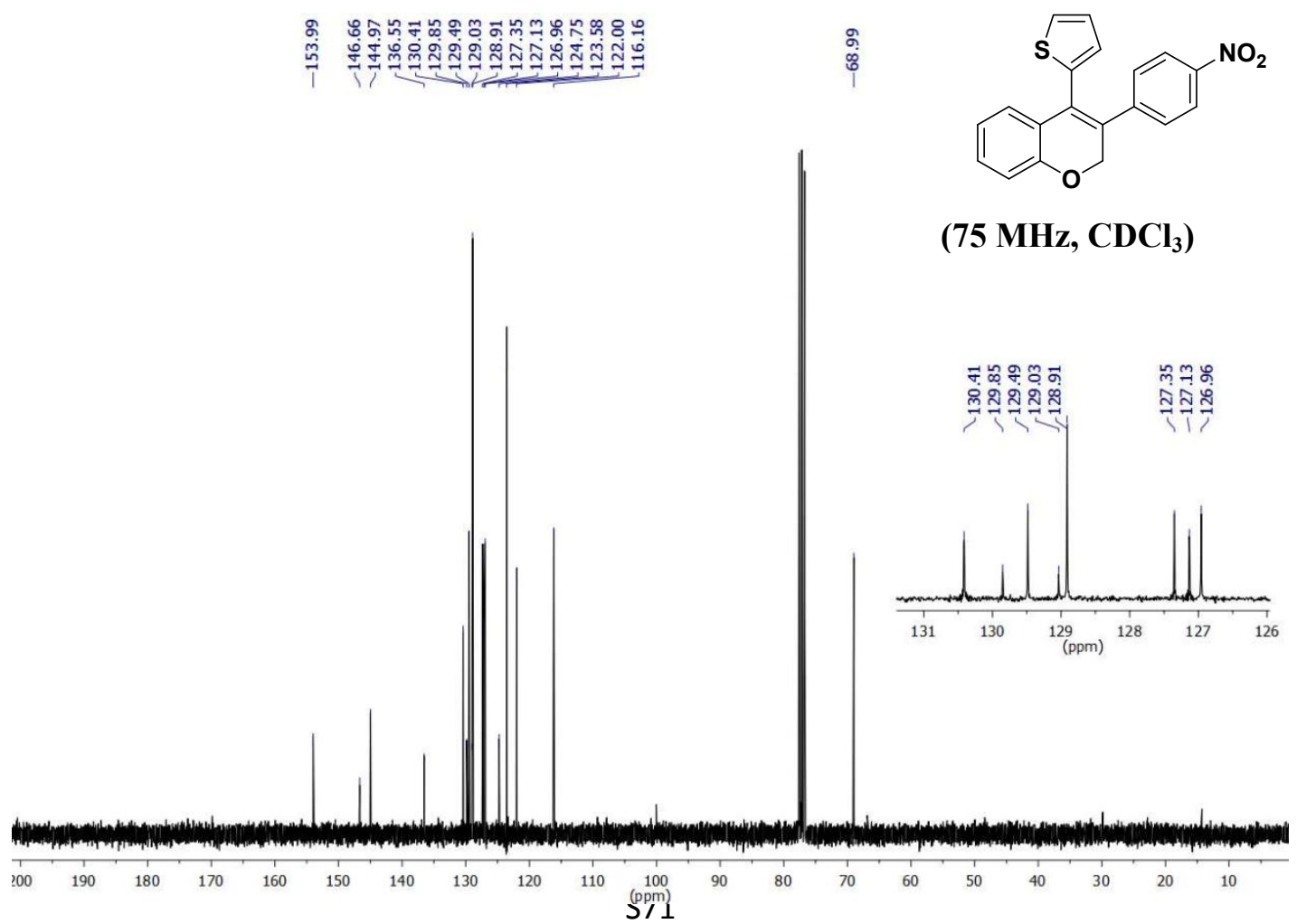
Figure S70. ${ }^{13} \mathrm{C}$ NMR of 3-(4-nitrophenyl)-4-(thiophen-2-yl)-2H-chromene (9c).

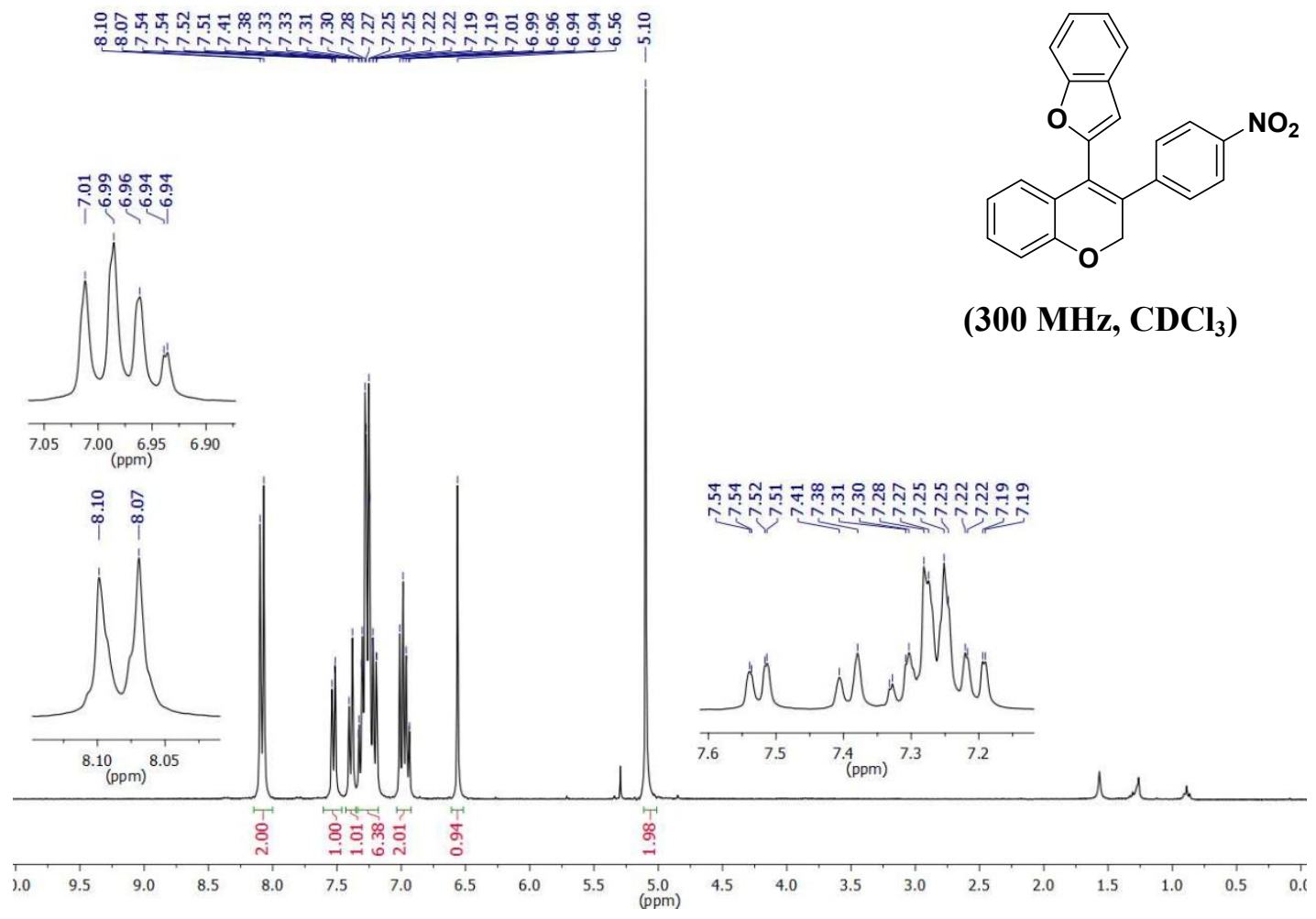

Figure S71. ${ }^{1} \mathrm{H}$ NMR of 4-(benzofuran-2-yl)-3-(4-nitrophenyl)-2 $H$-chromene (10c).

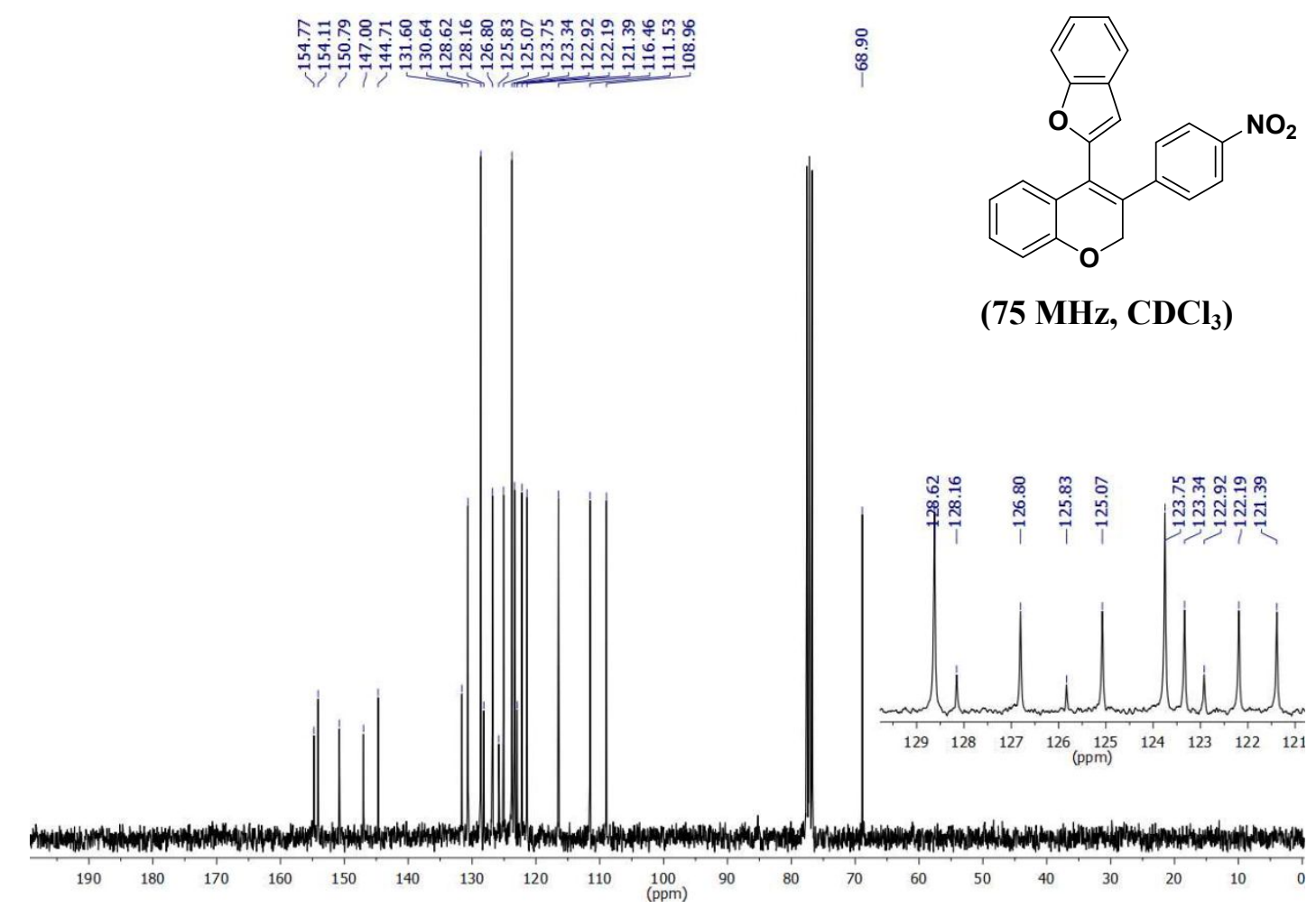

Figure S72. ${ }^{13} \mathrm{C}$ NMR of 4-(benzofuran-2-yl)-3-(4-nitrophenyl)-2 $H$-chromene (10c). 


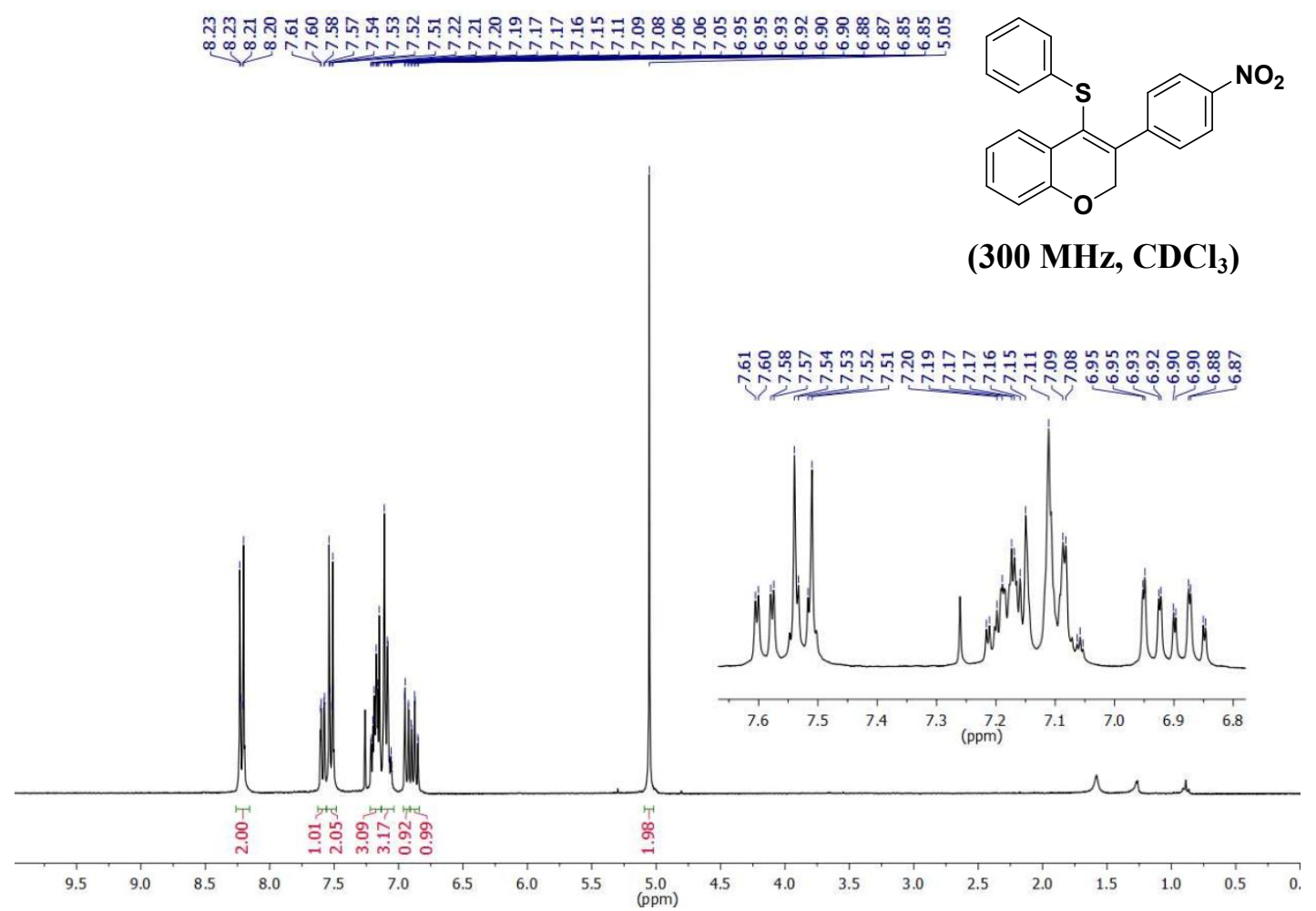

Figure S73. ${ }^{1} \mathrm{H}$ NMR of 3-(4-nitrophenyl)-4-(phenylthio)-2H-chromene (11c).

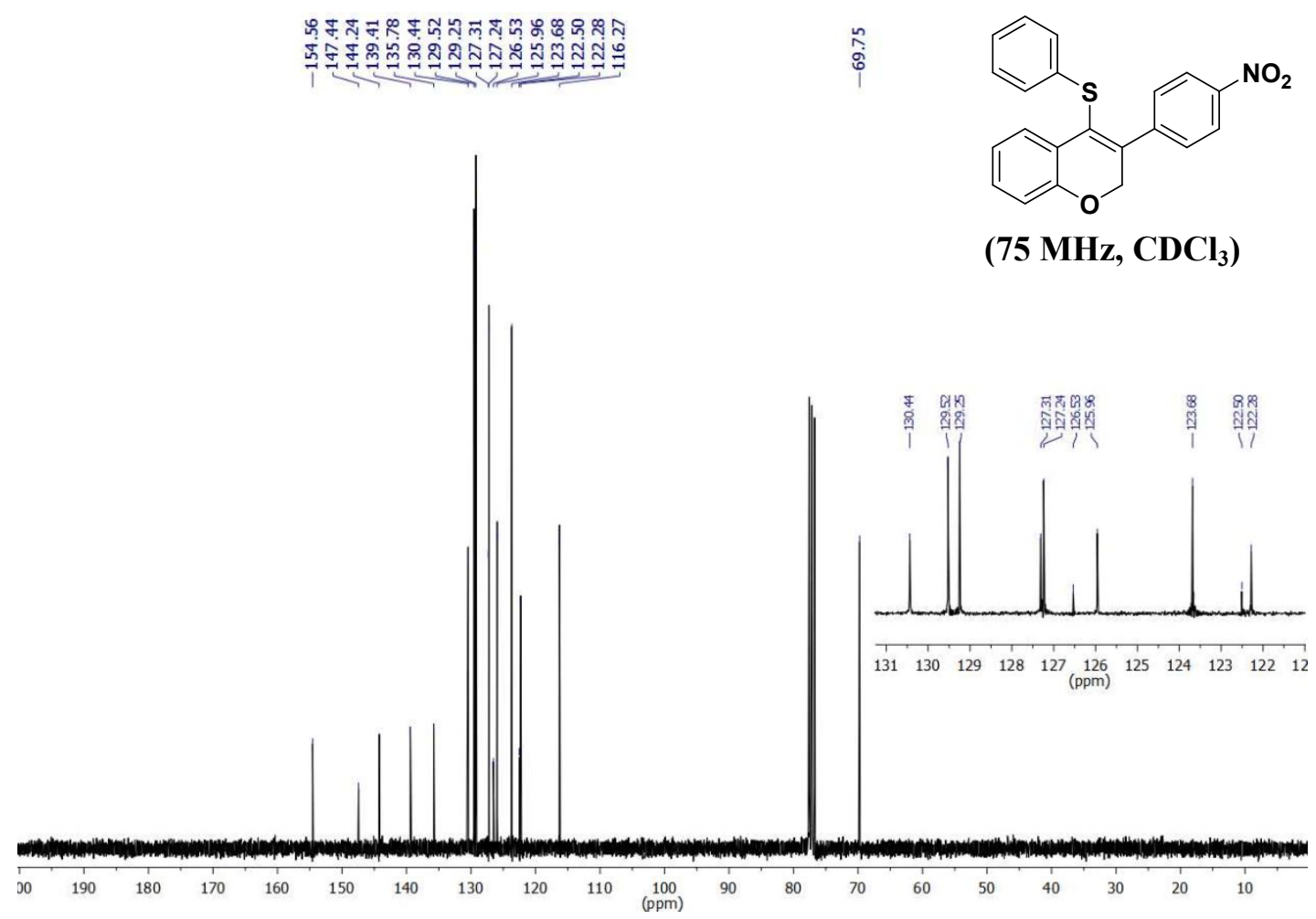

Figure S74. ${ }^{13} \mathrm{C}$ NMR of 3-(4-nitrophenyl)-4-(phenylthio)-2H-chromene (11c). 


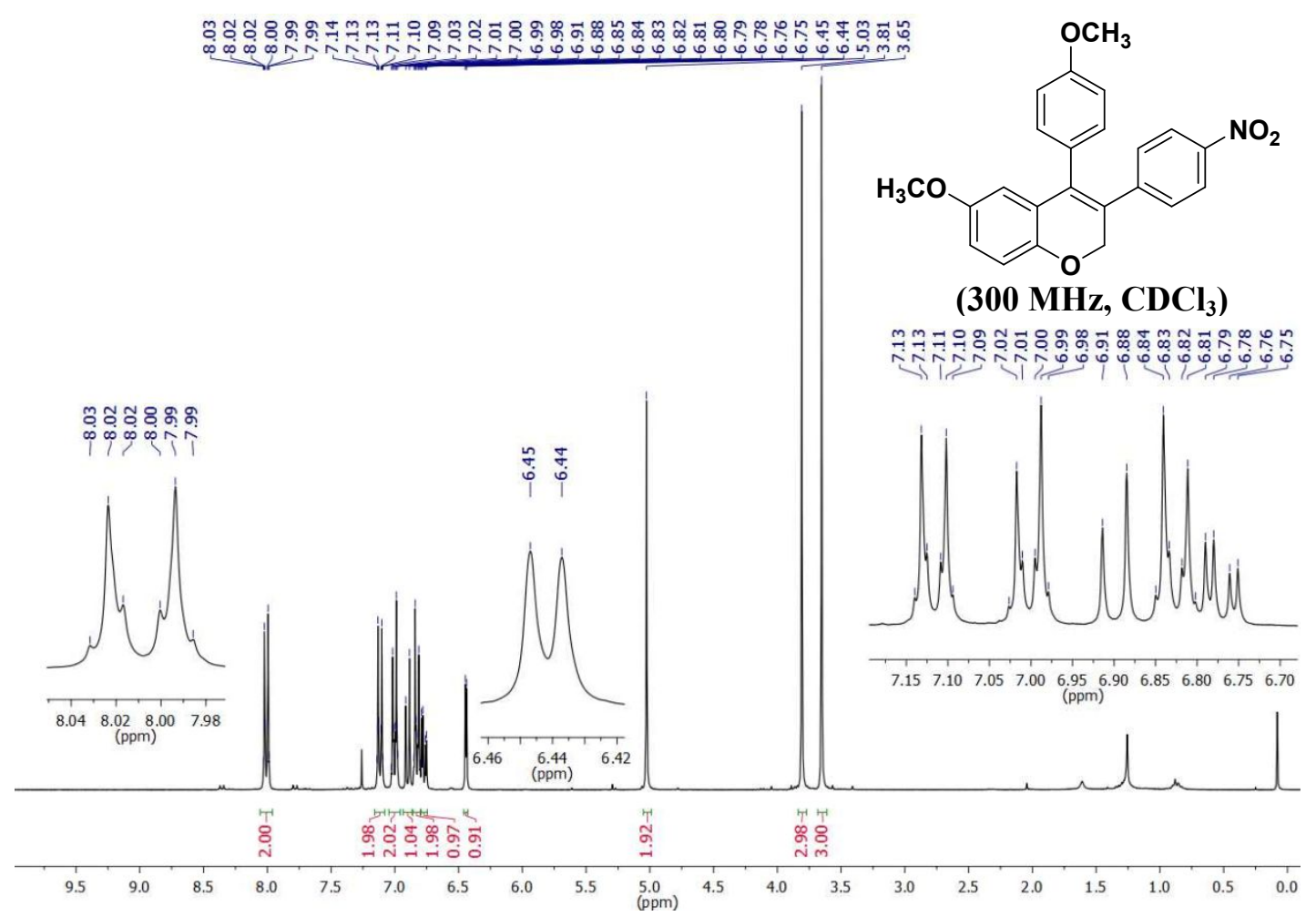

Figure S75. ${ }^{1} \mathrm{H}$ NMR of 6-methoxy-4-(4-methoxyphenyl)-3-(4-nitrophenyl)-2H-chromene (13c).

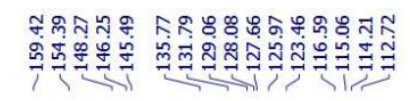

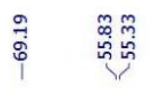

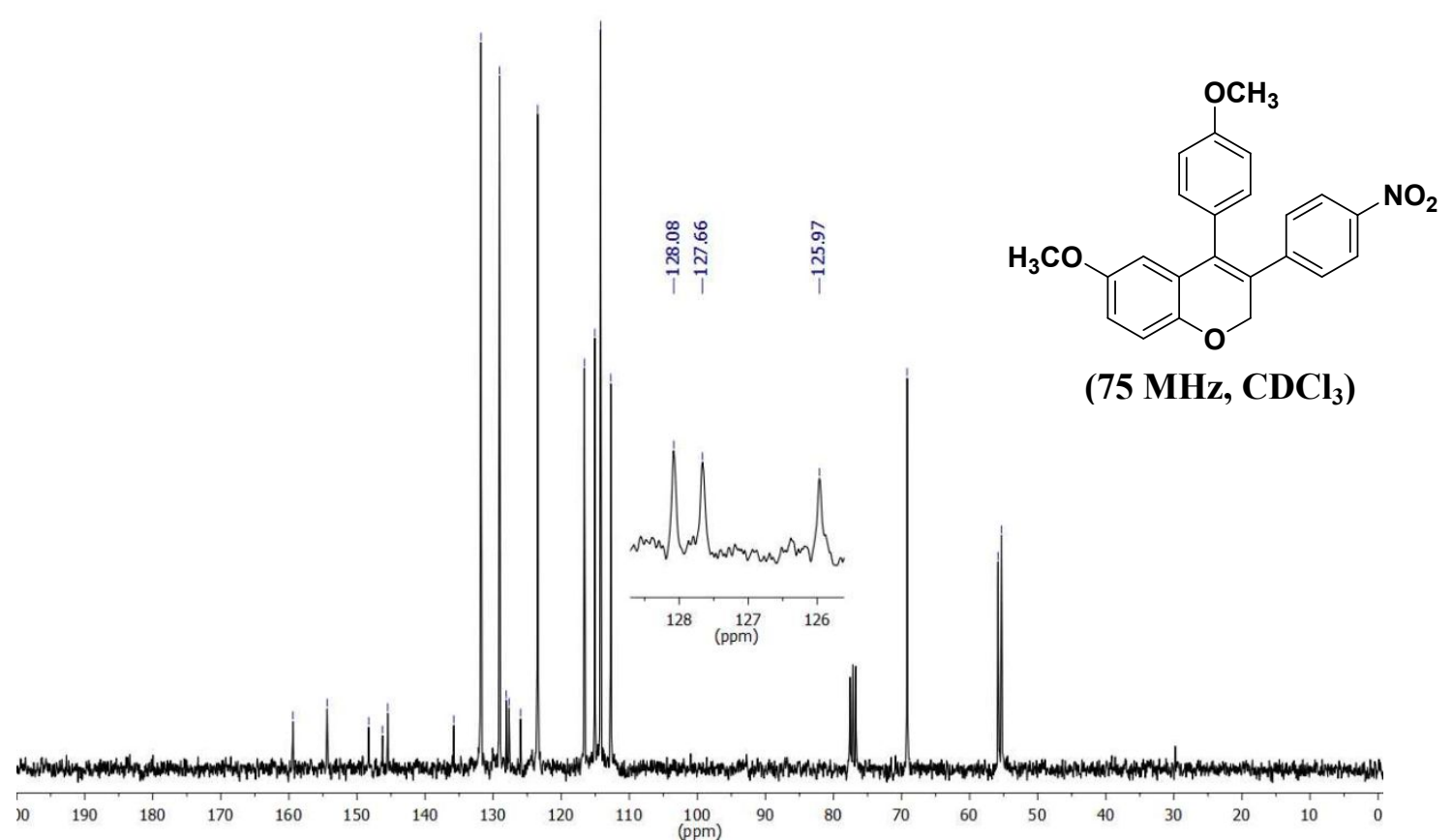

Figure S76. ${ }^{13} \mathrm{C}$ NMR of 6-methoxy-4-(4-methoxyphenyl)-3-(4-nitrophenyl)-2H-chromene (13c). 


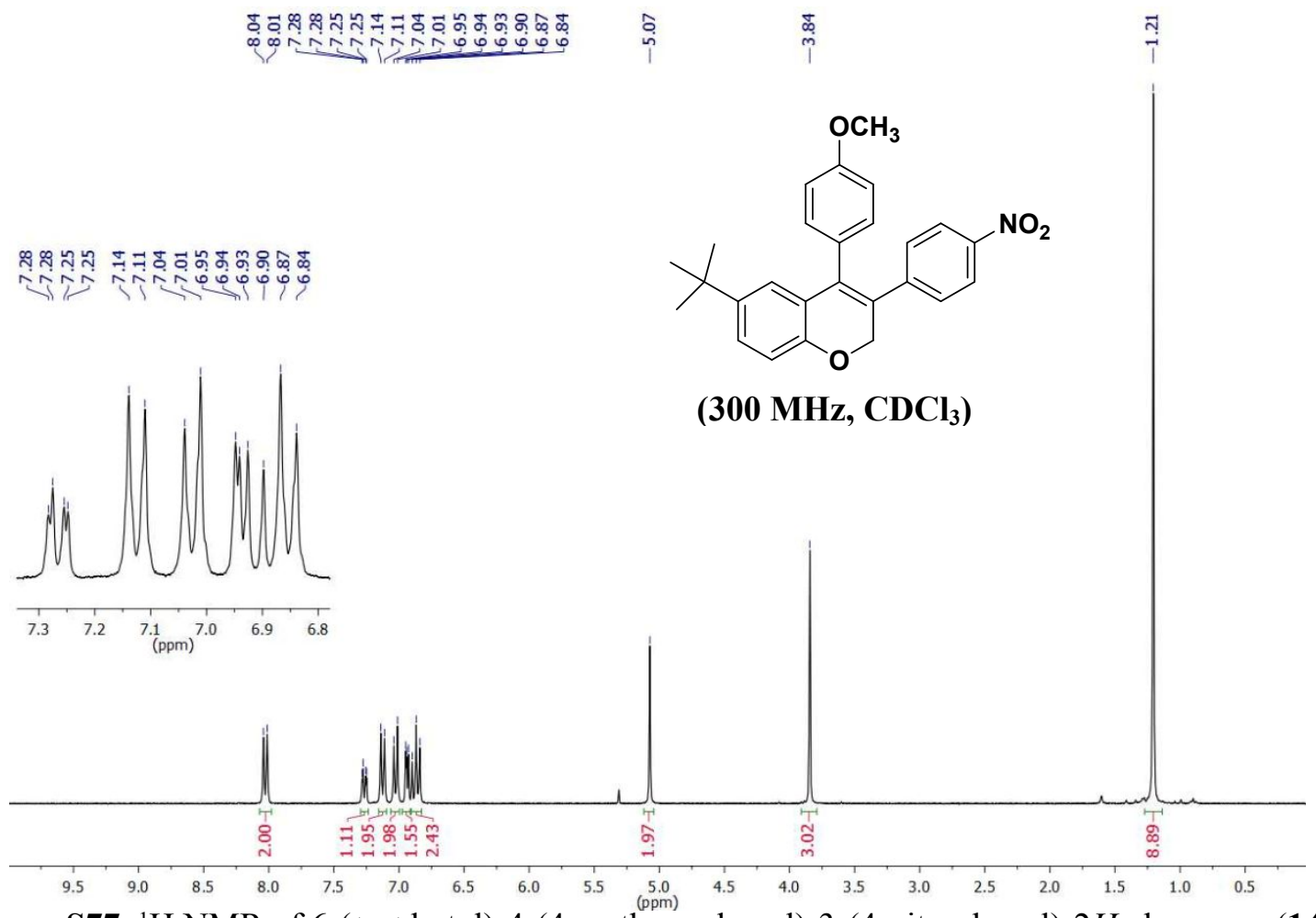

Figure S77. ${ }^{1} \mathrm{H}$ NMR of 6-(tert-butyl)-4-(4-methoxyphenyl)-3-(4-nitrophenyl)-2H-chromene (14c).

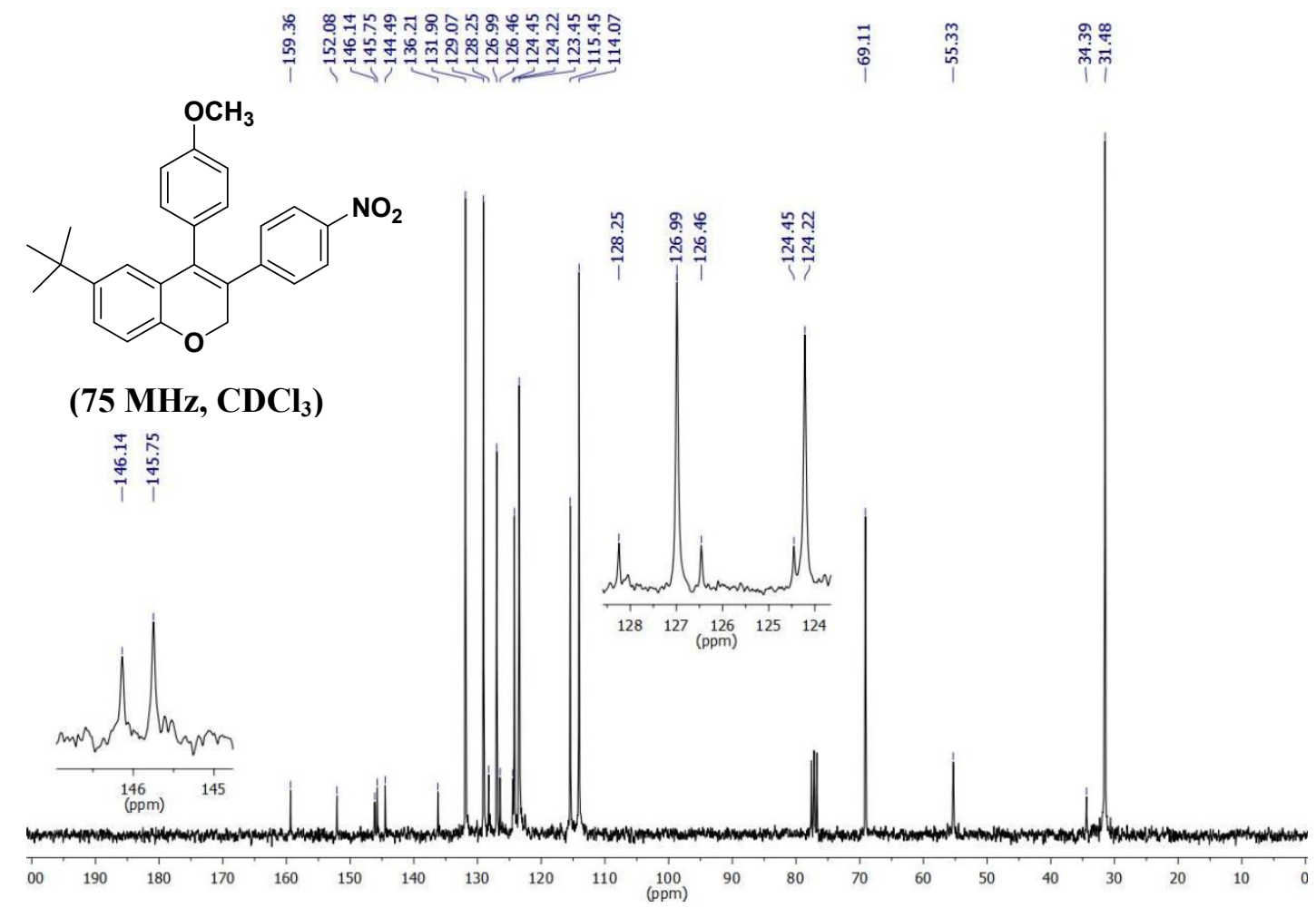

Figure S78. ${ }^{13} \mathrm{C}$ NMR of 6-(tert-butyl)-4-(4-methoxyphenyl)-3-(4-nitrophenyl)-2 $H$-chromene (14c). 


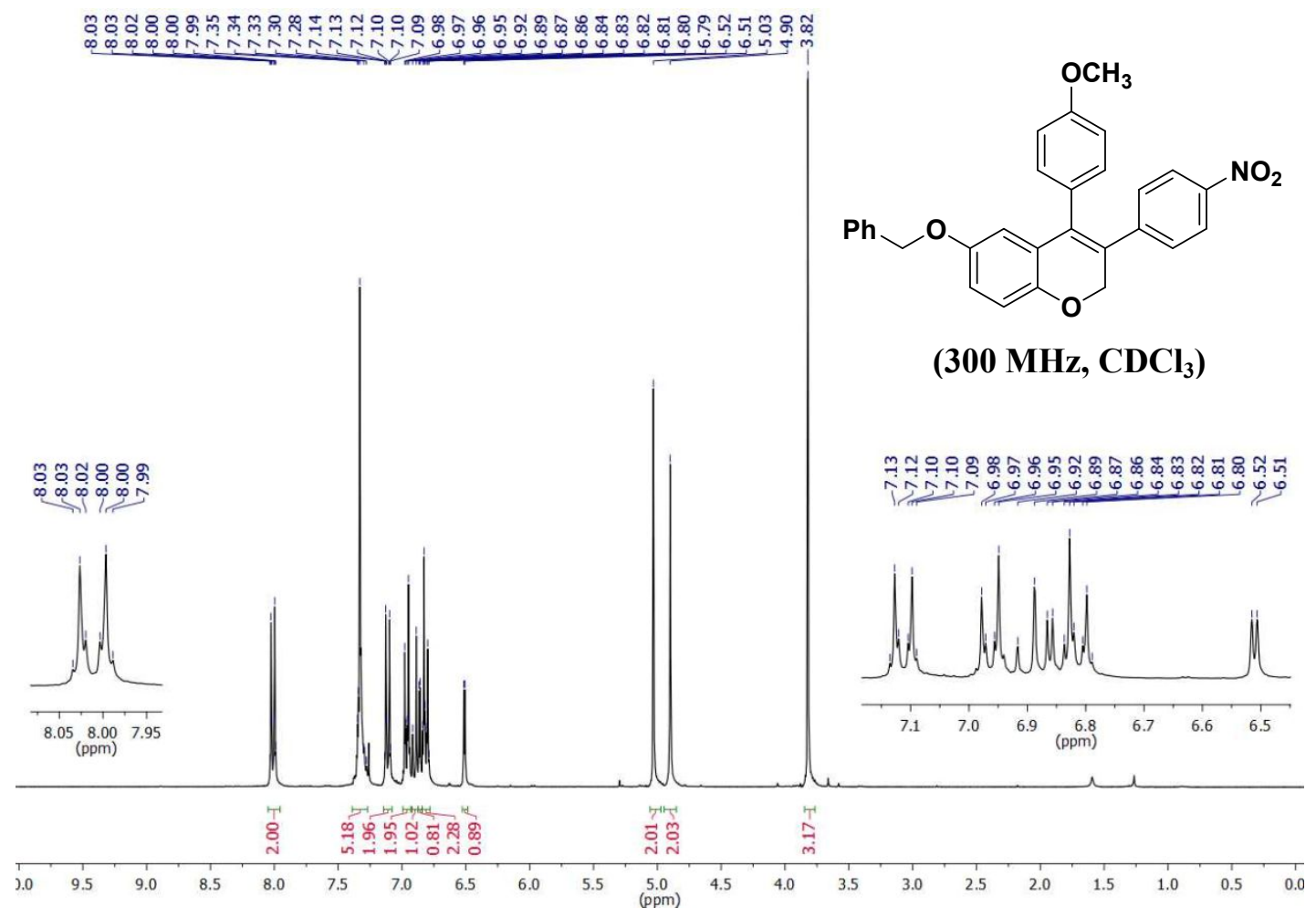

Figure S79. ${ }^{1} \mathrm{H}$ NMR of 6-(benzyloxy)-4-(4-methoxyphenyl)-3-(4-nitrophenyl)-2H-chromene (15c).

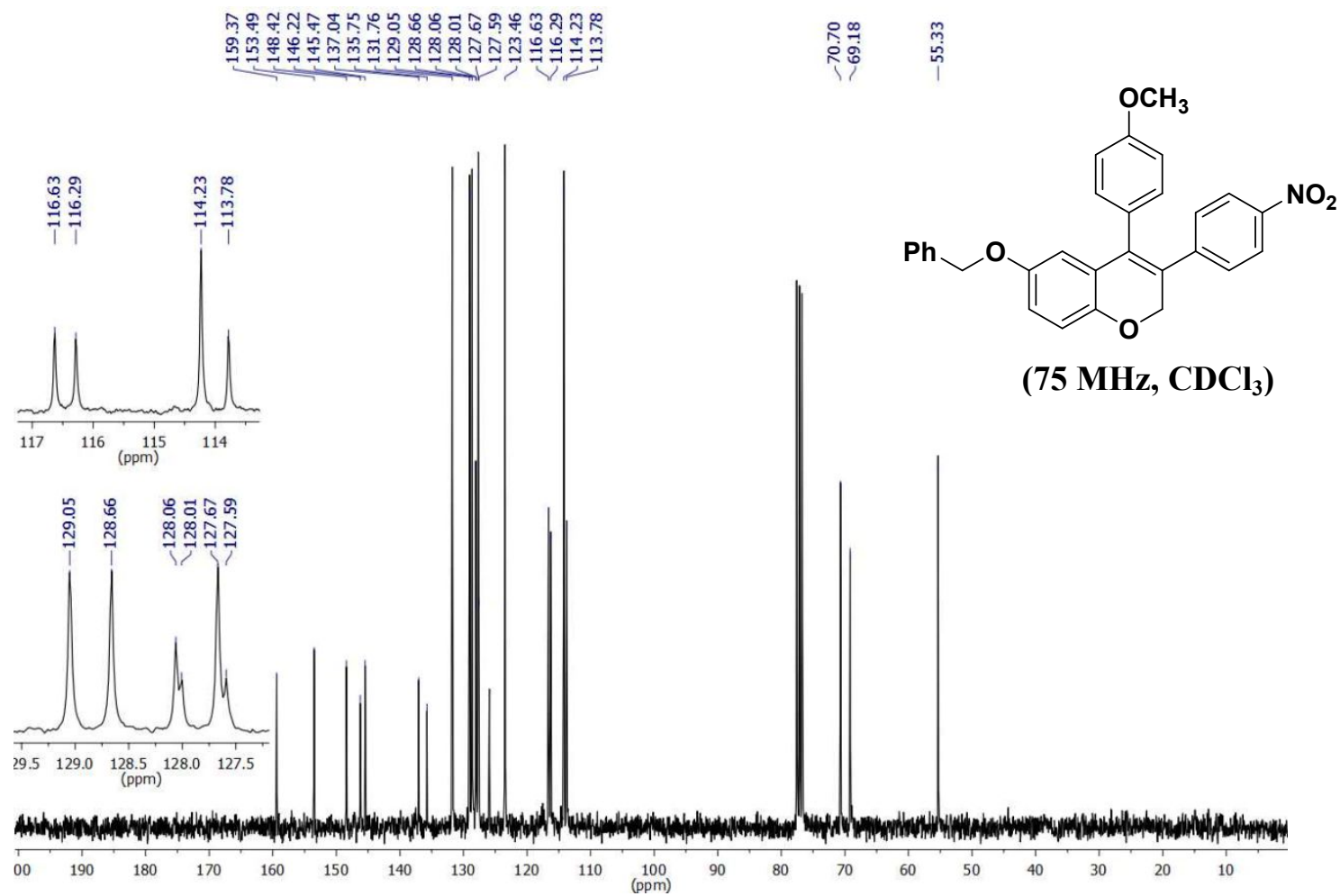


Figure S80. ${ }^{13} \mathrm{C}$ NMR of 6-(benzyloxy)-4-(4-methoxyphenyl)-3-(4-nitrophenyl)-2 $H$-chromene (15c).

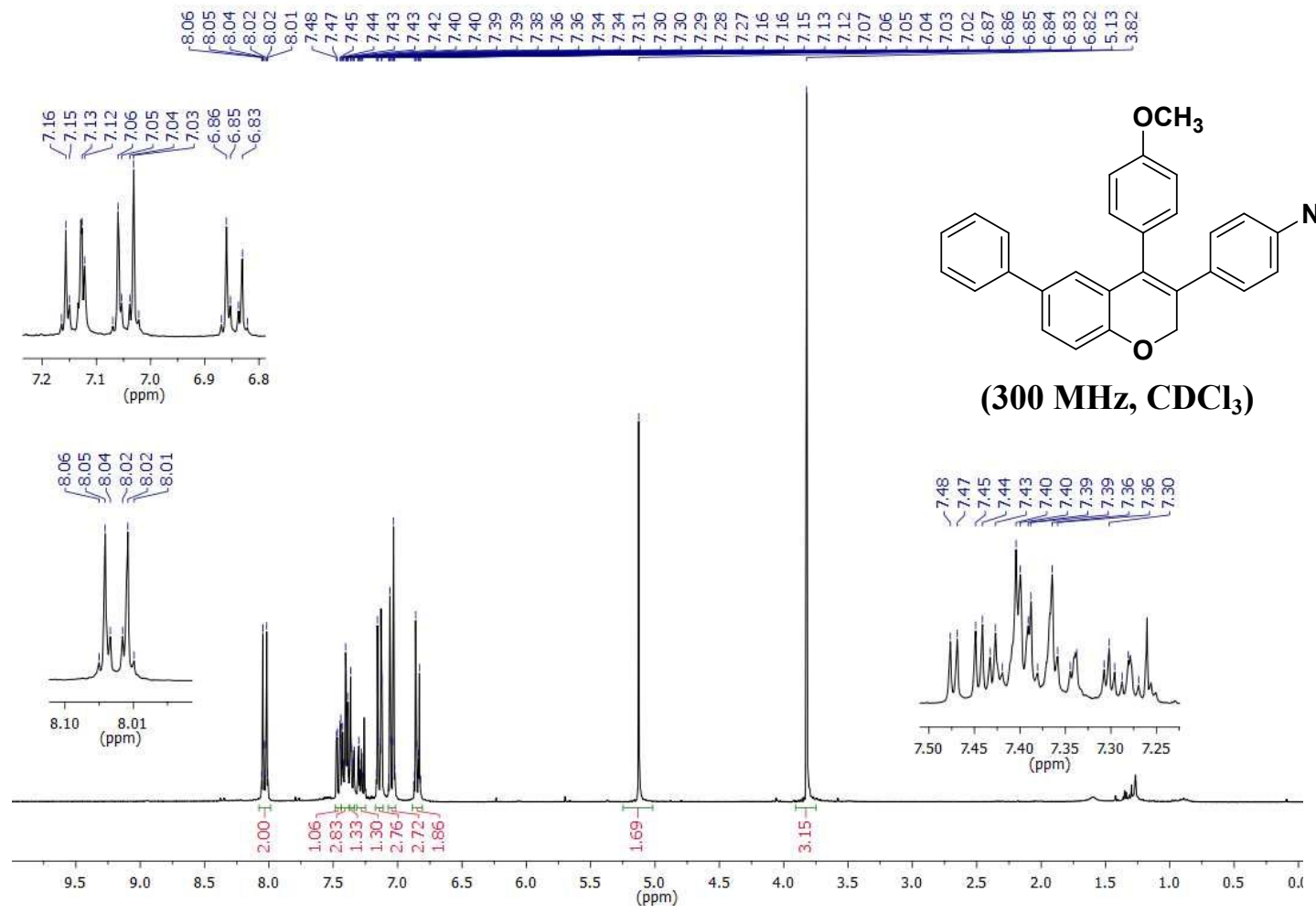

Figure S81. ${ }^{1} \mathrm{H}$ NMR of 4-(4-methoxyphenyl)-3-(4-nitrophenyl)-6-phenyl-2H-chromene (16c).

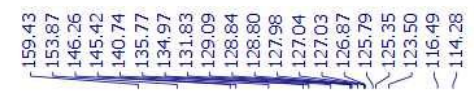

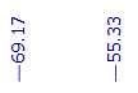
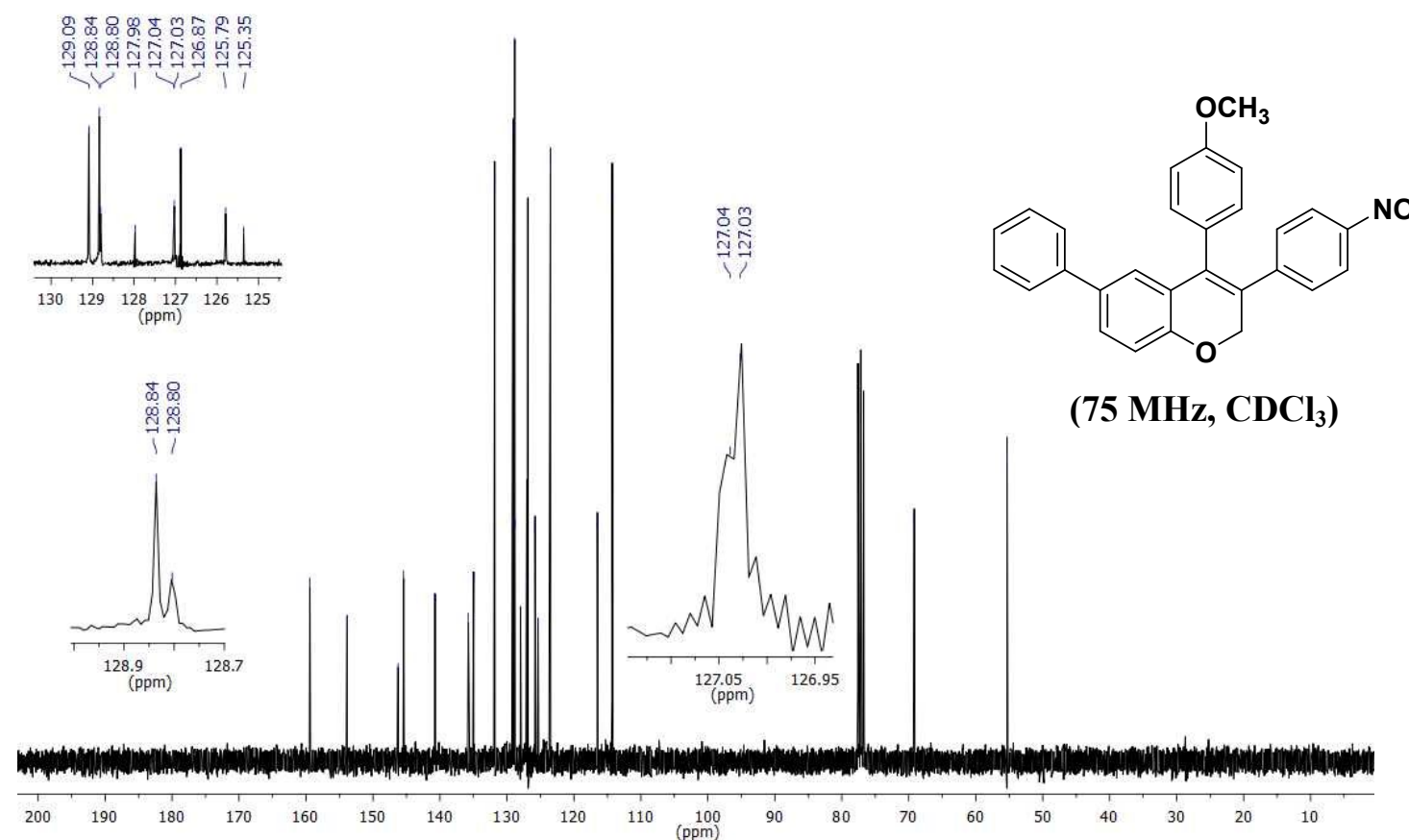

Figure S82. ${ }^{13} \mathrm{C}$ NMR of 4-(4-methoxyphenyl)-3-(4-nitrophenyl)-6-phenyl-2H-chromene (16c). 


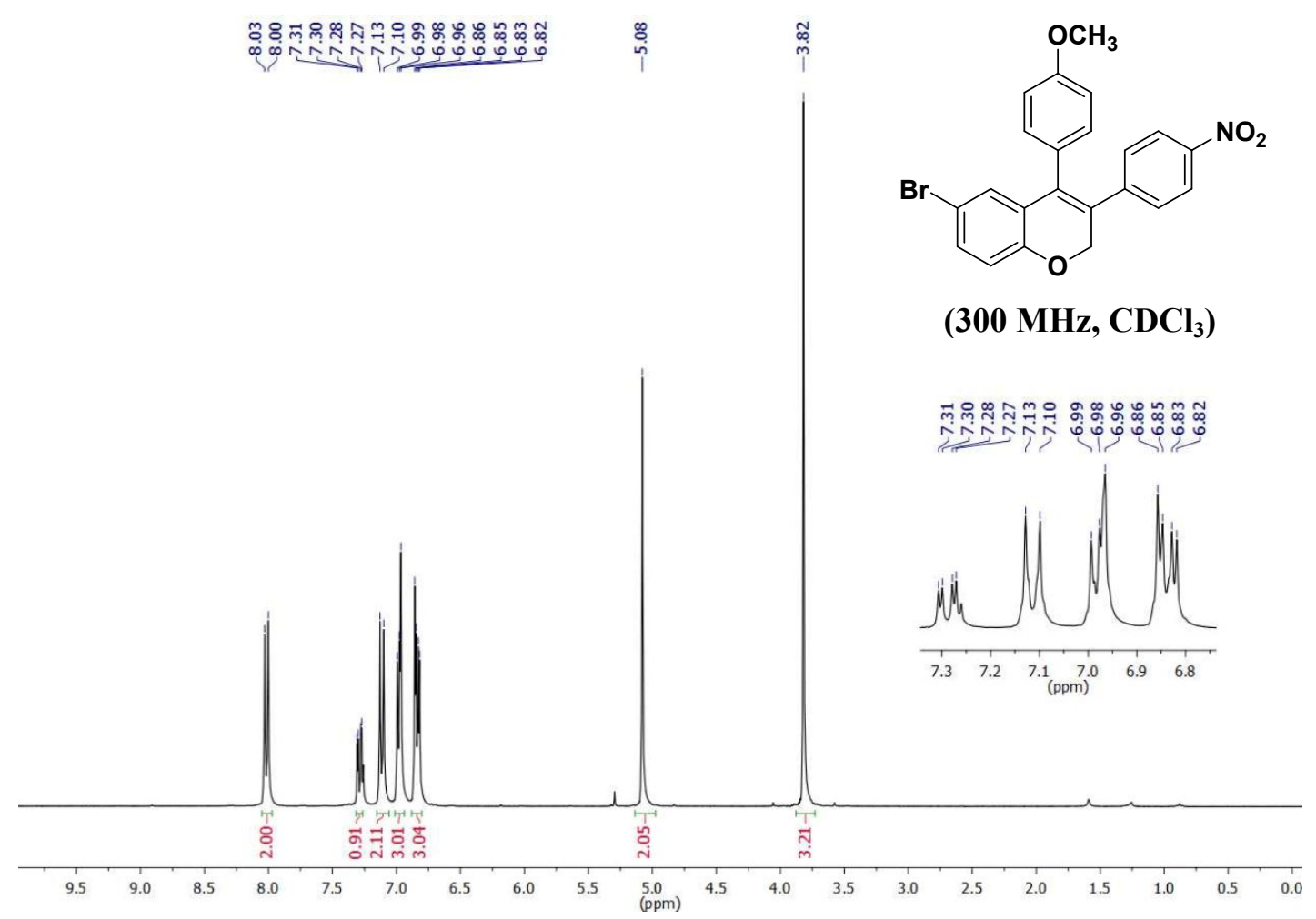

Figure S83. ${ }^{1} \mathrm{H}$ NMR of 6-bromo-4-(4-methoxyphenyl)-3-(4-nitrophenyl)-2H-chromene (17c).

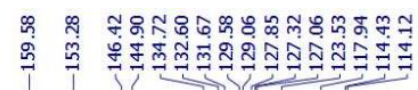

용
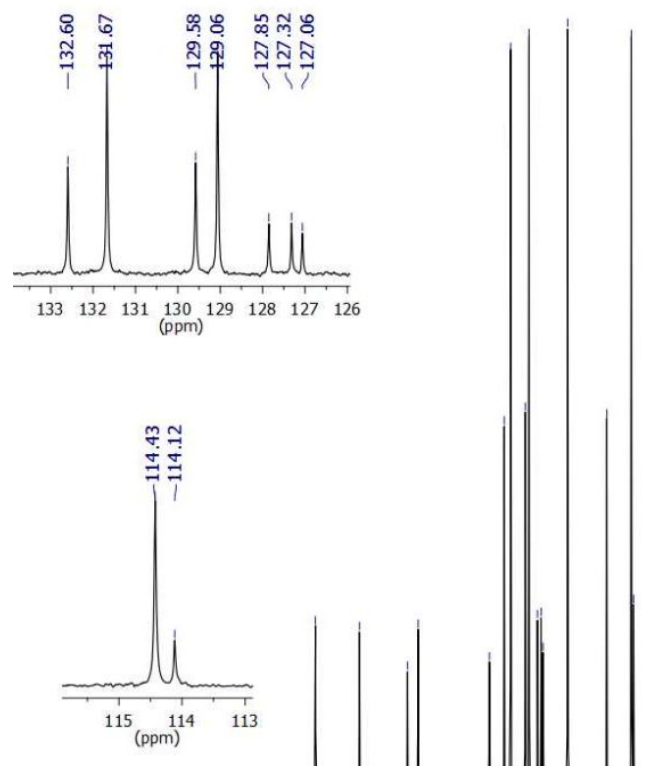<smiles>COc1ccc(C2=C(c3ccc([N+](=O)[O-])cc3)COc3ccc(Br)cc32)cc1</smiles>
(75 $\left.\mathrm{MHz}, \mathrm{CDCl}_{3}\right)$
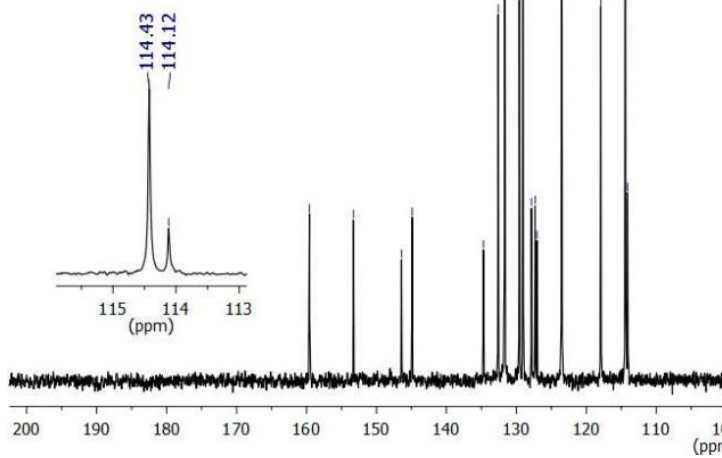

Figure S84. ${ }^{13} \mathrm{C}$ NMR of 6-bromo-4-(4-methoxyphenyl)-3-(4-nitrophenyl)-2H-chromene (17c). 


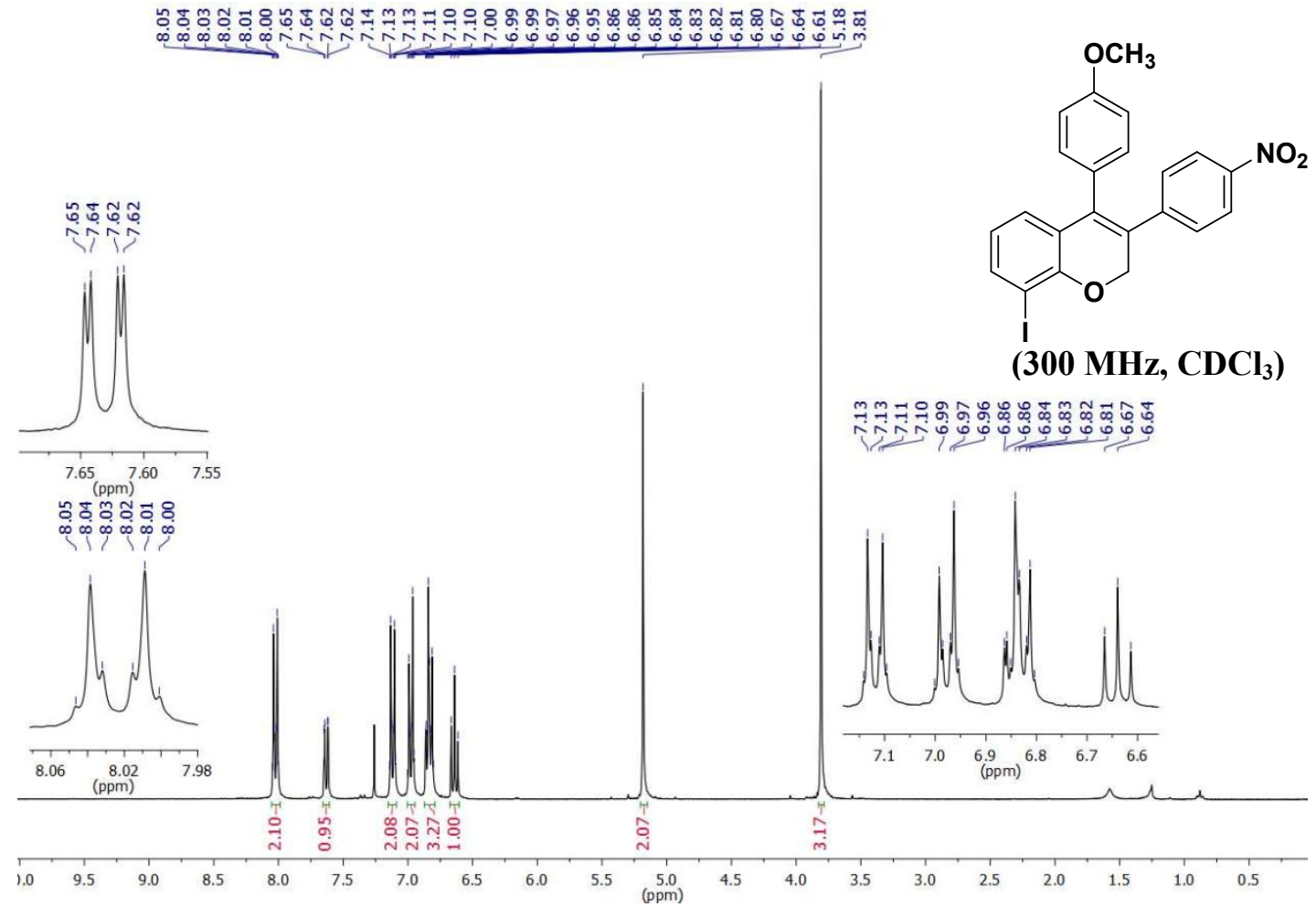

Figure S85. ${ }^{1} \mathrm{H}$ NMR of 8-iodo-4-(4-methoxyphenyl)-3-(4-nitrophenyl)-2H-chromene (18c).

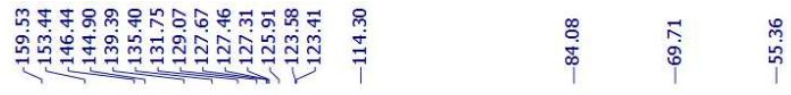<smiles>COc1ccc(C2=C(c3ccc([N+](=O)[O-])cc3)COc3c(I)cccc32)cc1</smiles>

(75 $\left.\mathrm{MHz}, \mathrm{CDCl}_{3}\right)$
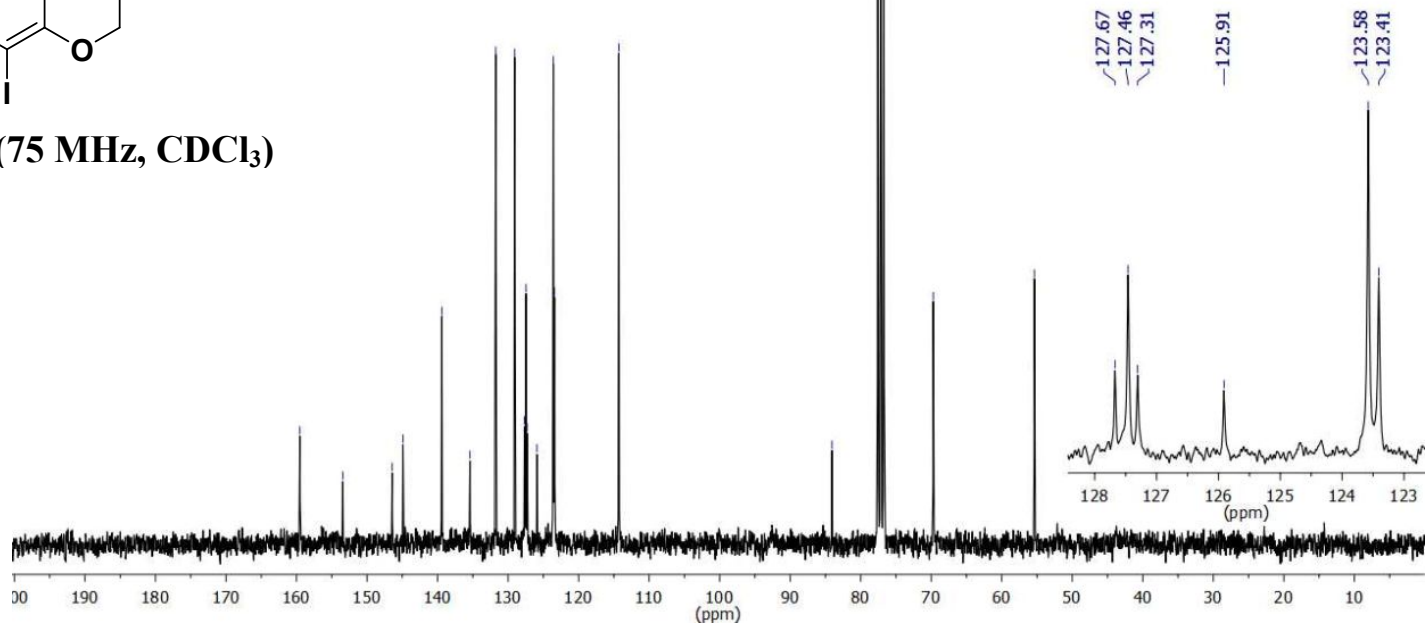

Figure S86. ${ }^{13} \mathrm{C}$ NMR of 8-iodo-4-(4-methoxyphenyl)-3-(4-nitrophenyl)-2H-chromene (18c). 


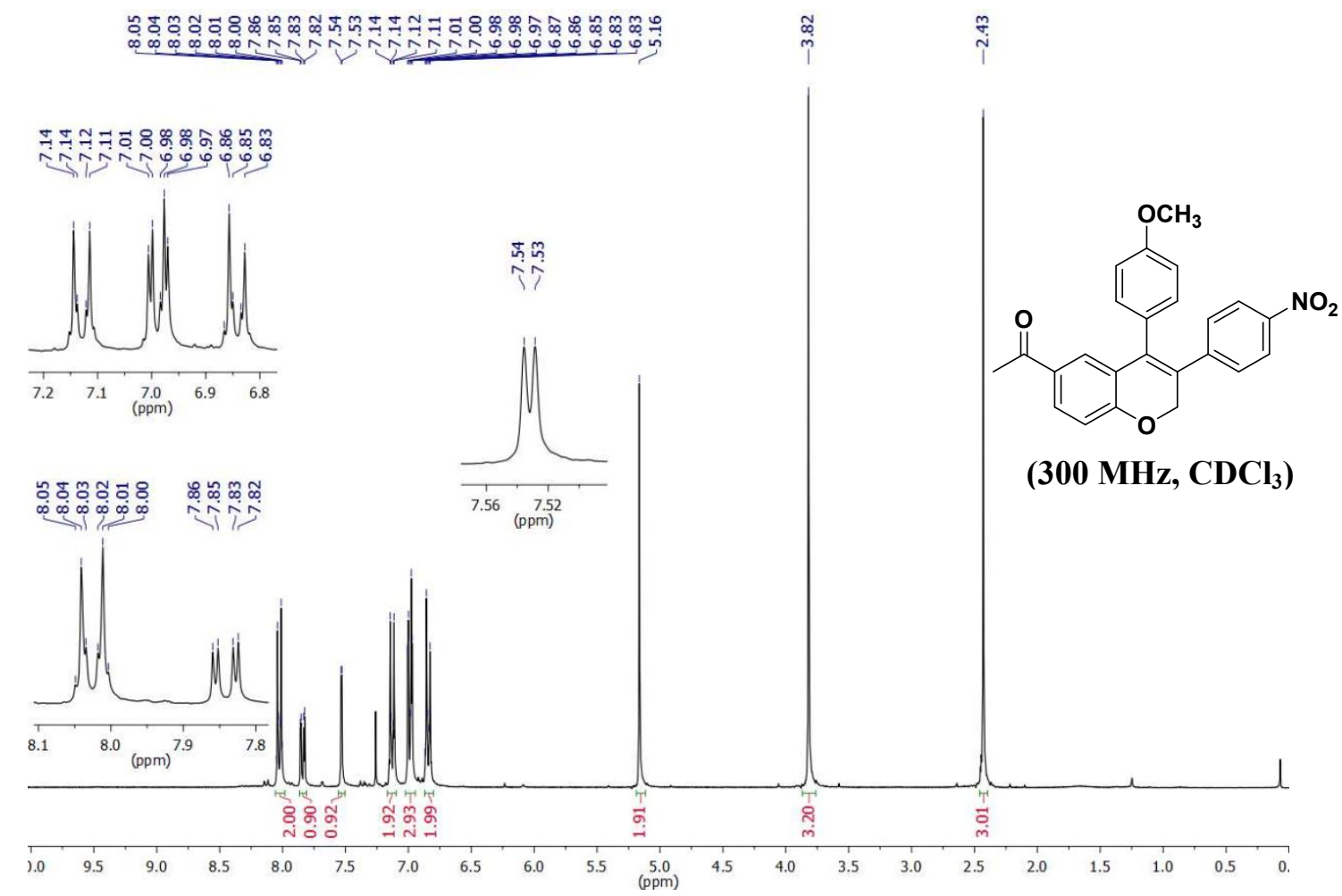

Figure S87. ${ }^{1} \mathrm{H}$ NMR of 1-(4-(4-methoxyphenyl)-3-(4-nitrophenyl)-2H-chromen-6-yl)ethan-1-one (19c).
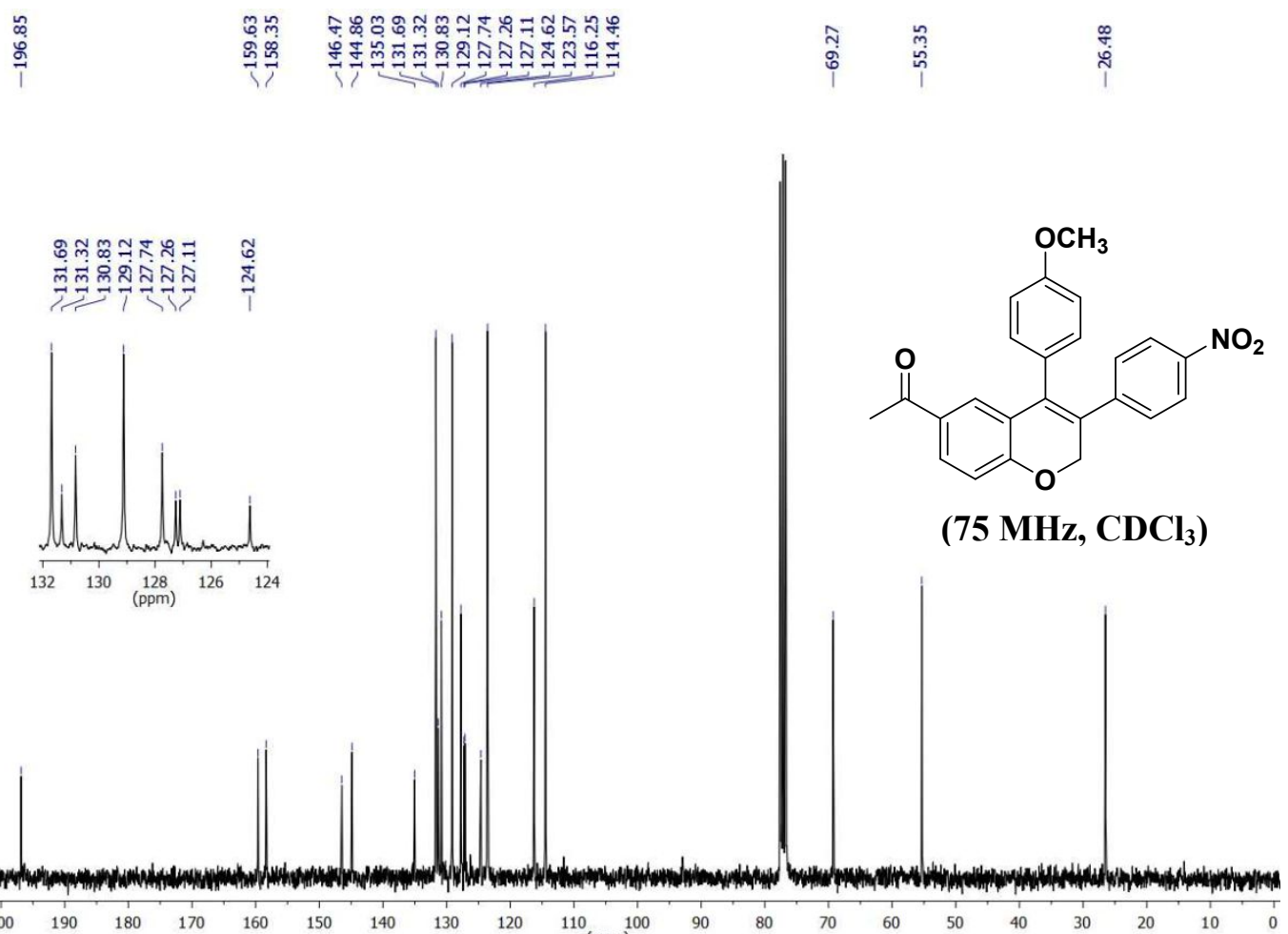

Figure S88. ${ }^{13} \mathrm{C}$ NMR of 1-(4-(4-methoxyphenyl)-3-(4-nitrophenyl)-2H-chromen-6-yl)ethan-1-one (19c). 


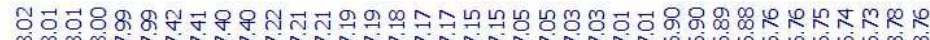

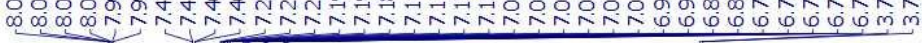<smiles>COc1ccc(C2=C(c3ccc([N+](=O)[O-])cc3)CSc3ccccc32)cc1</smiles>

(400 MHz, $\left.\mathrm{CDCl}_{3}\right)$

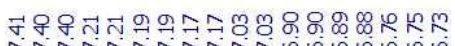

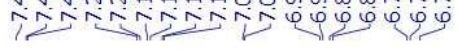
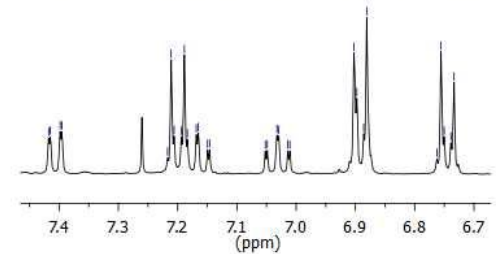
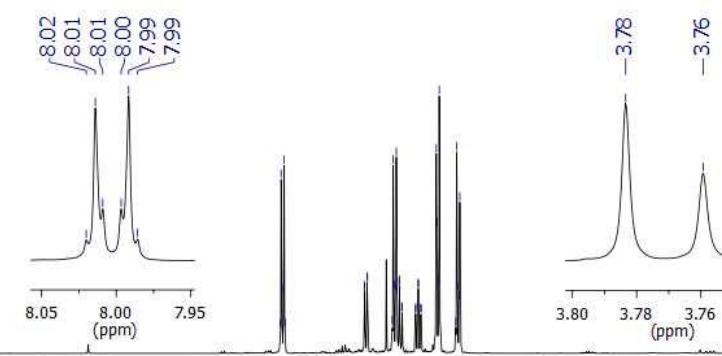

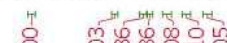

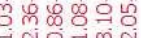

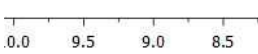

Figure S89. ${ }^{1} \mathrm{H}$ NMR of 4-(4-methoxyphenyl)-3-(4-nitrophenyl)-2 $H$-thiochromene (20c).

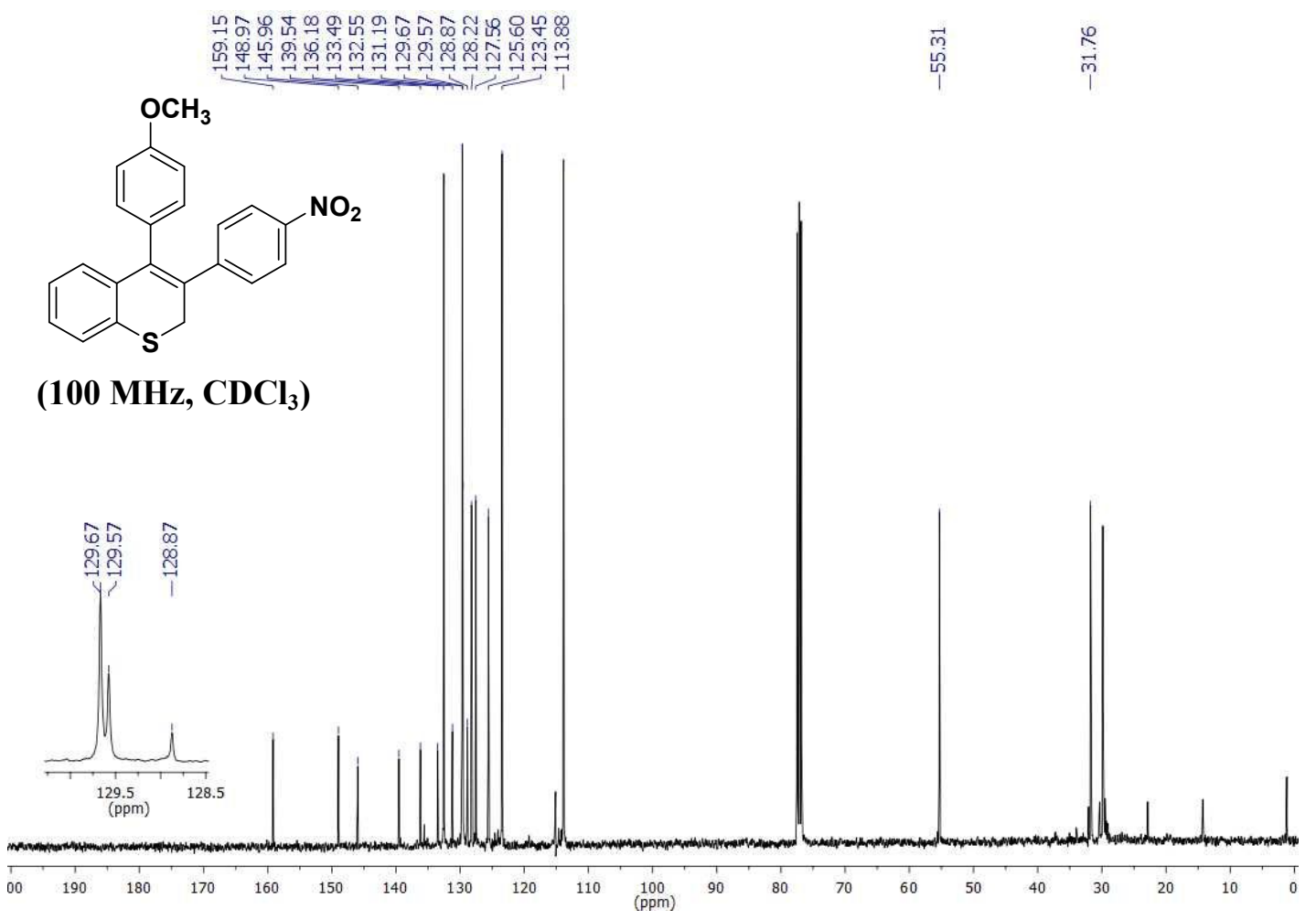

Figure S90. ${ }^{13} \mathrm{C}$ NMR of 4-(4-methoxyphenyl)-3-(4-nitrophenyl)-2 $H$-thiochromene (20c). 


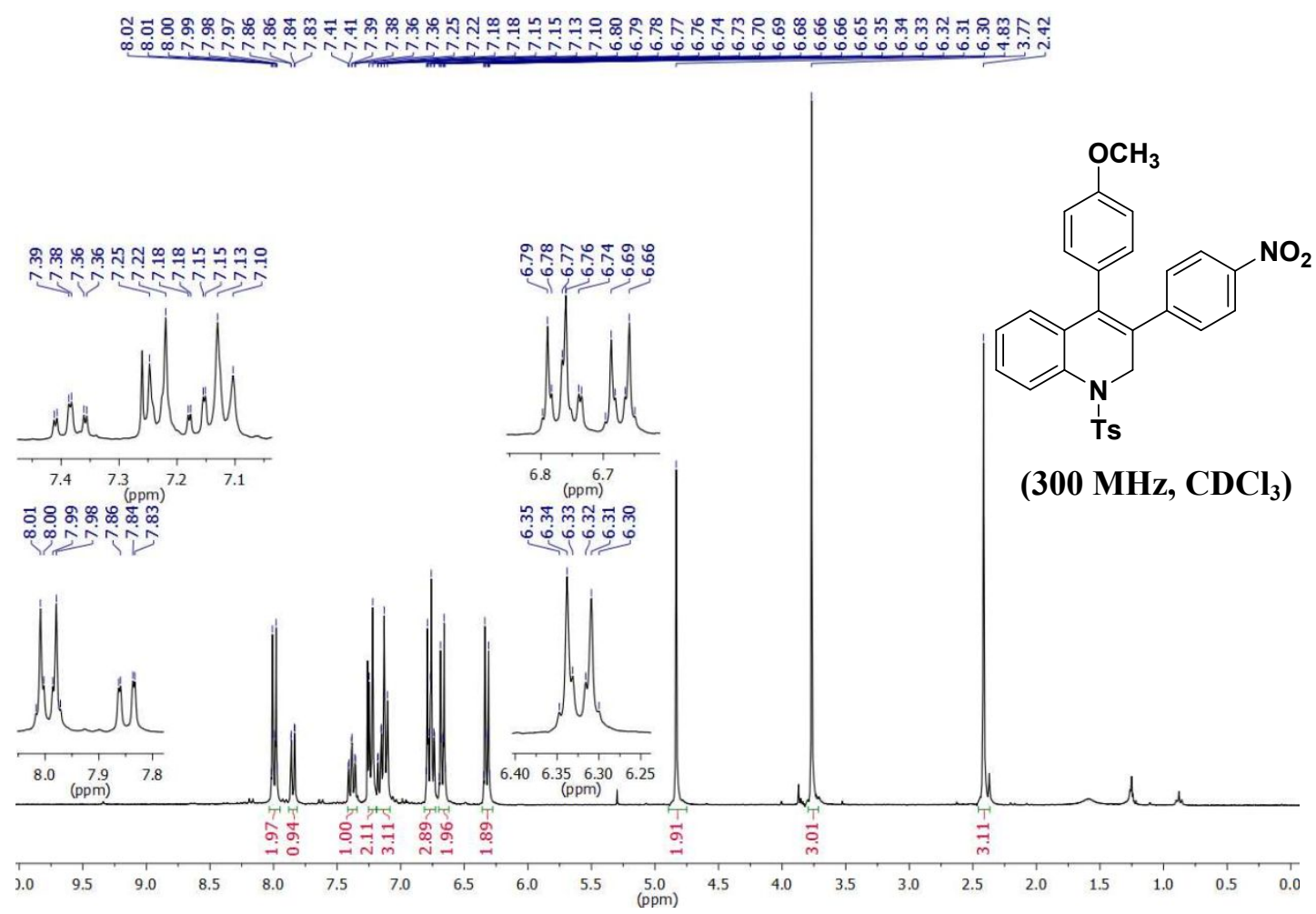

Figure S91. ${ }^{1} \mathrm{H}$ NMR of 4-(4-methoxyphenyl)-3-(4-nitrophenyl)-1-tosyl-1,2-dihydroquinoline (21c).

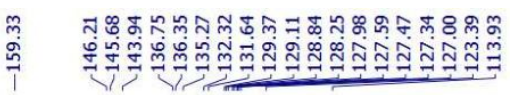

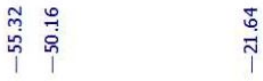

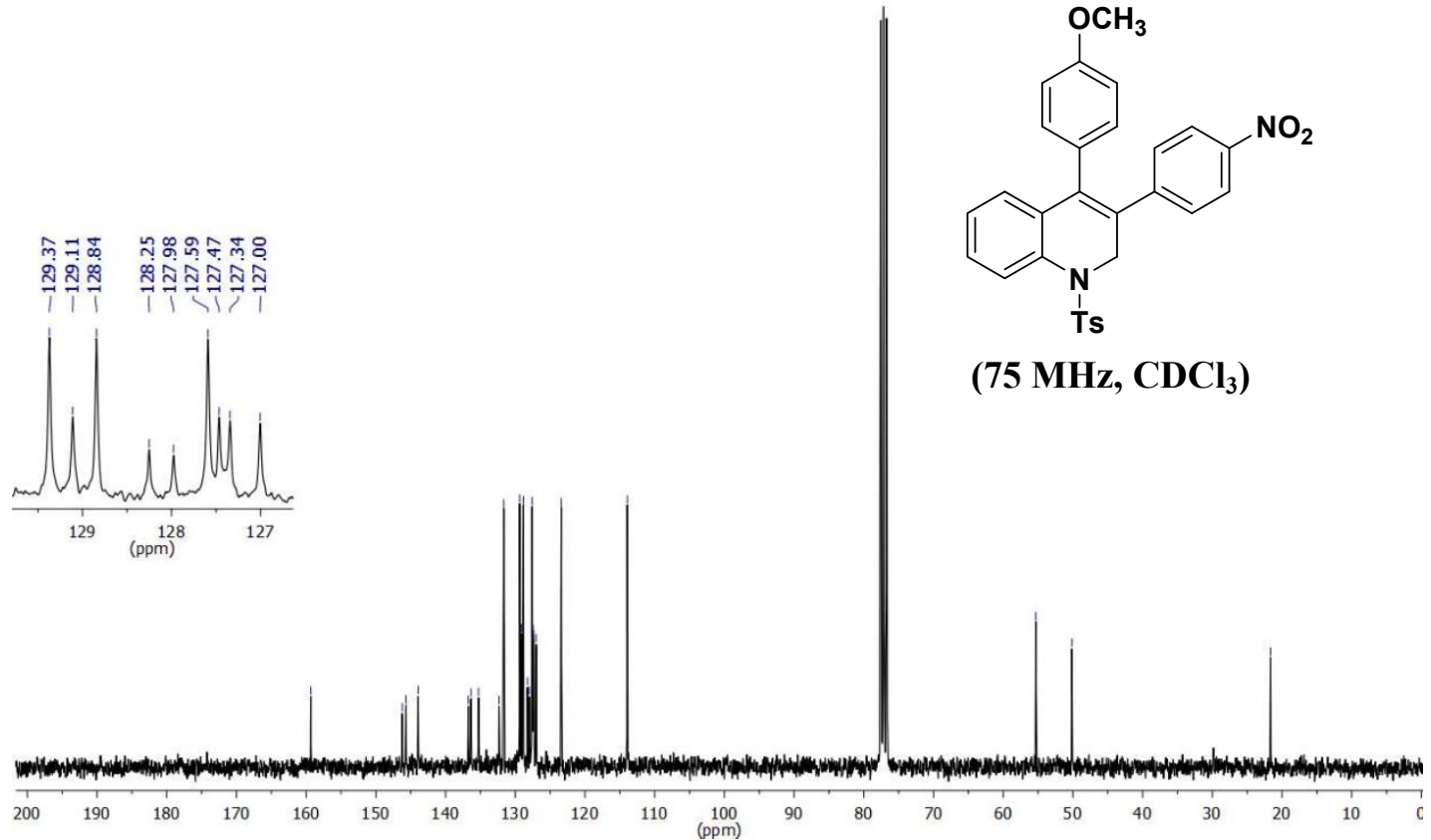

Figure S92. ${ }^{13} \mathrm{C}$ NMR of 4-(4-methoxyphenyl)-3-(4-nitrophenyl)-1-tosyl-1,2-dihydroquinoline (21c). 


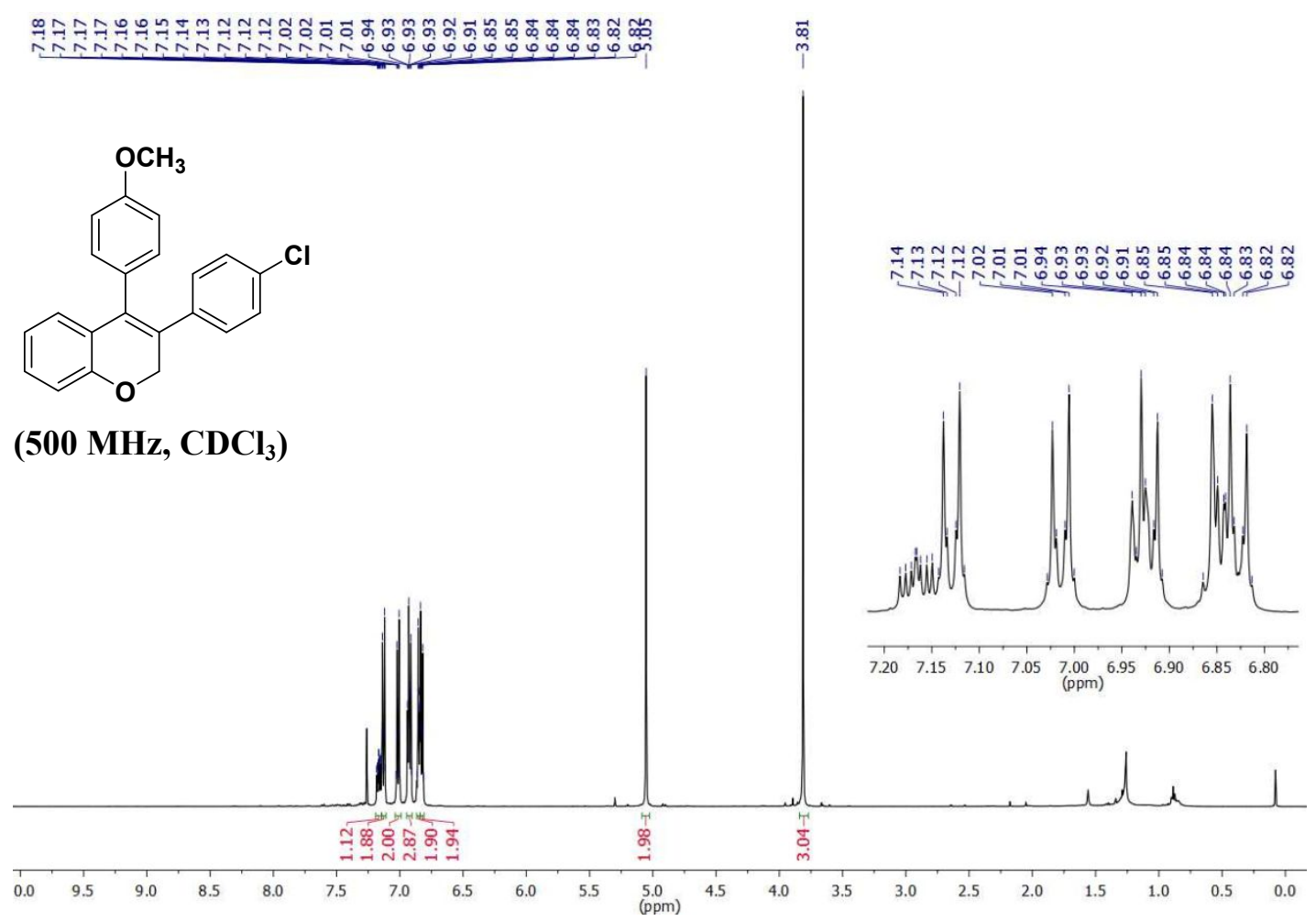

Figure S93. ${ }^{1} \mathrm{H}$ NMR of 3-(4-chlorophenyl)-4-(4-methoxyphenyl)-2H-chromene (22c).
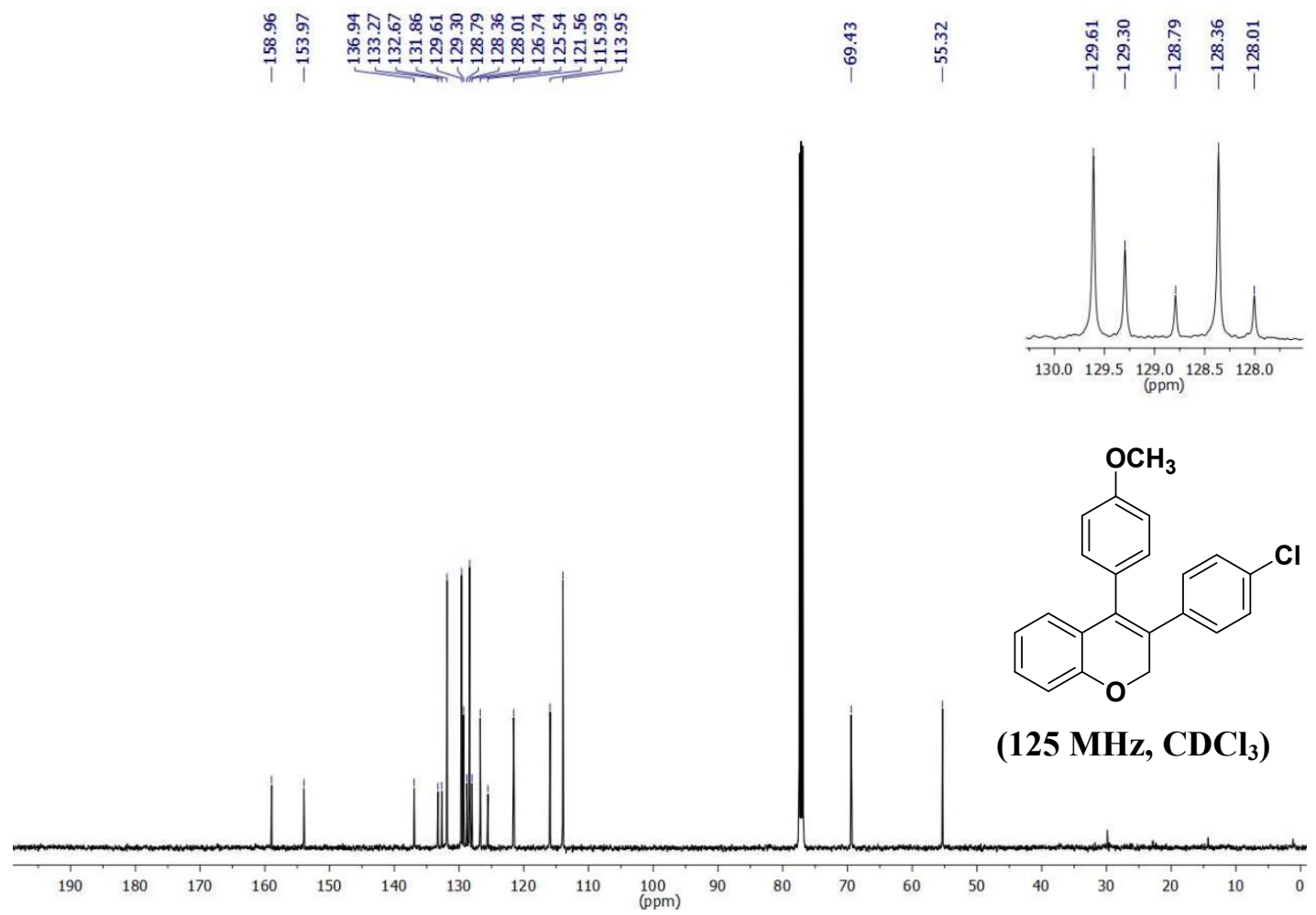

Figure S94. ${ }^{13} \mathrm{C}$ NMR of 3-(4-chlorophenyl)-4-(4-methoxyphenyl)-2 $H$-chromene (22c). 


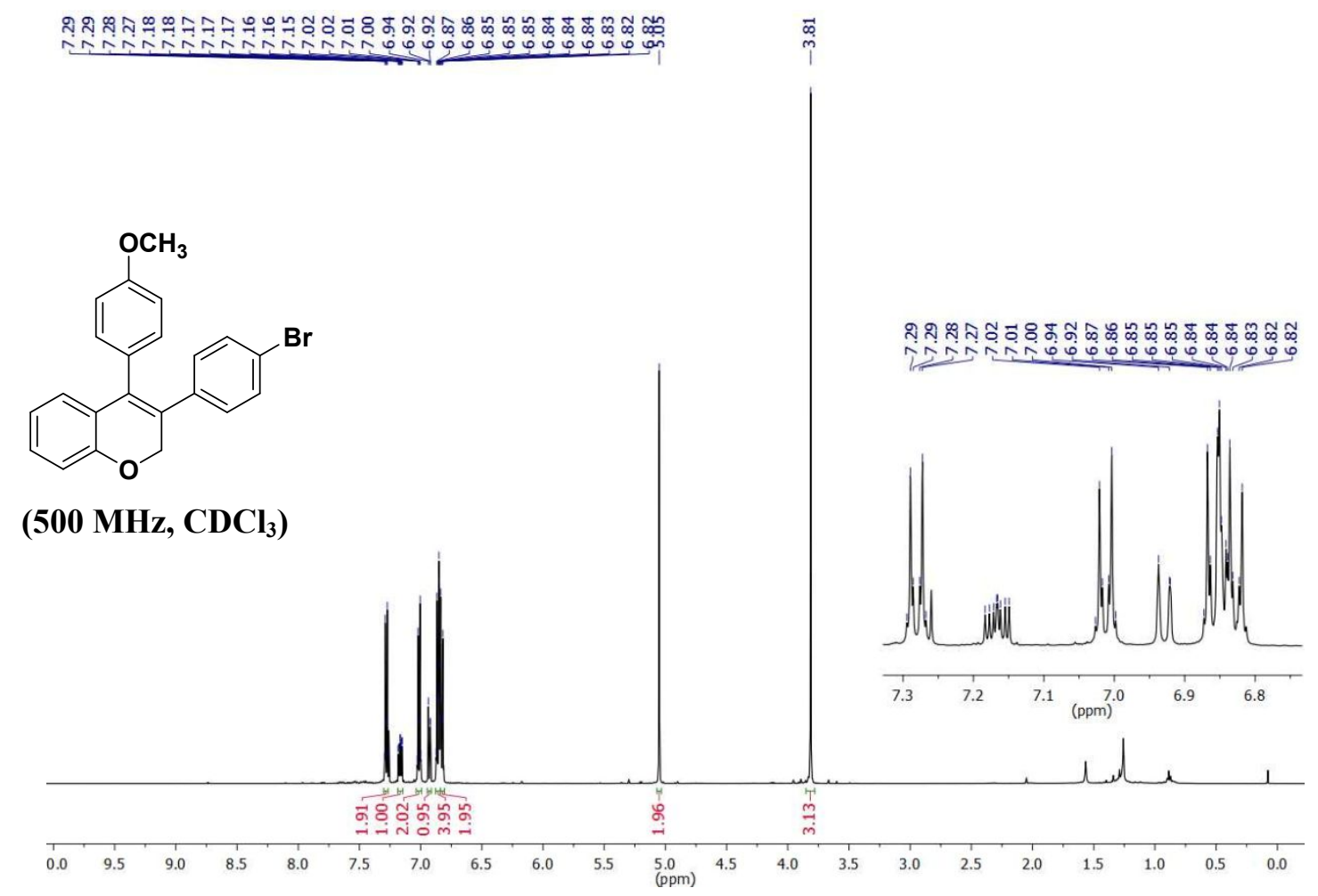

Figure S95. ${ }^{1} \mathrm{H}$ NMR of 3-(4-bromophenyl)-4-(4-methoxyphenyl)-2H-chromene (23c).
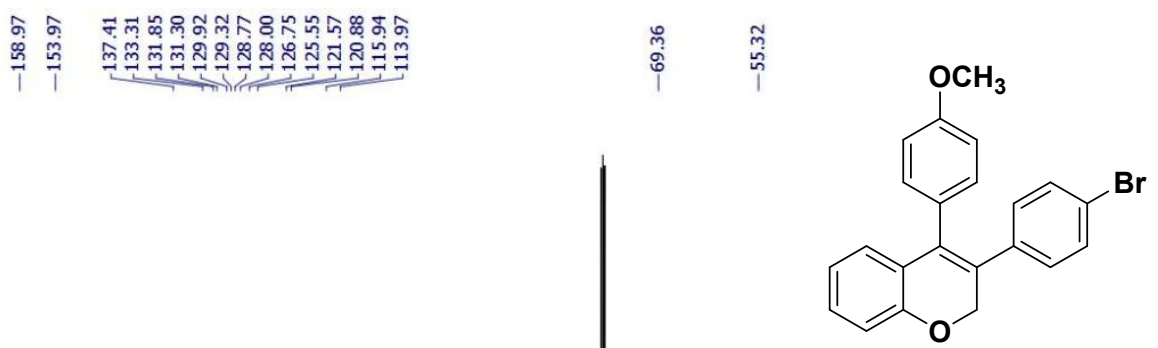

(125 MHz, $\left.\mathrm{CDCl}_{3}\right)$

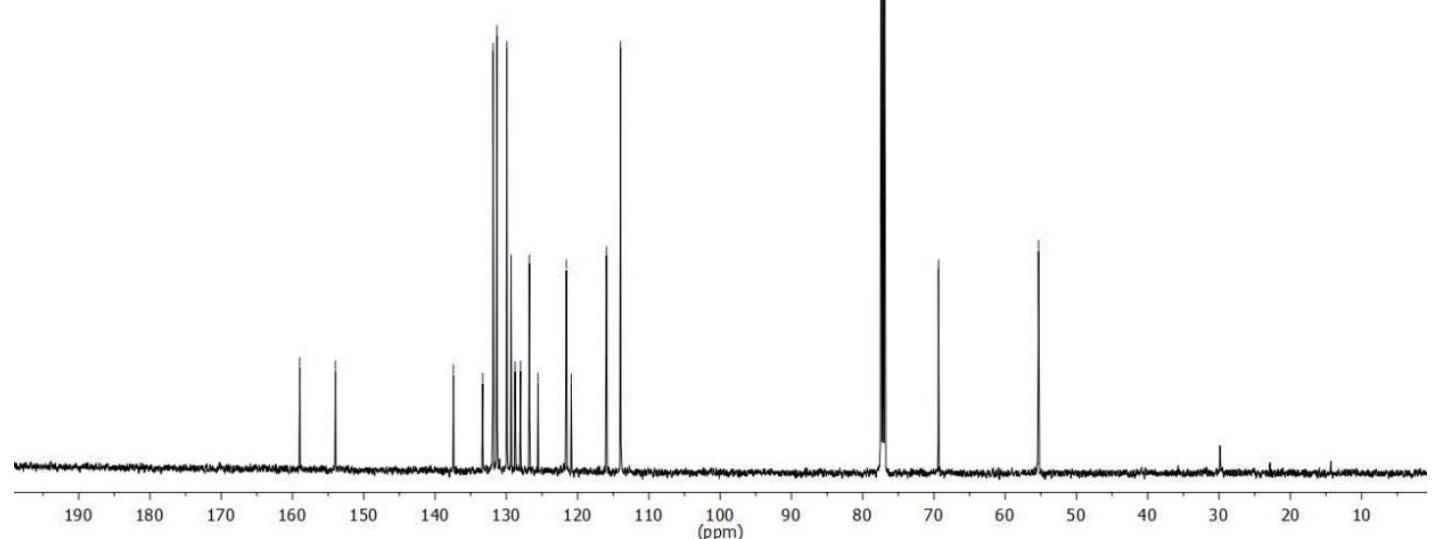

Figure S96. ${ }^{13} \mathrm{C}$ NMR of 3-(4-bromophenyl)-4-(4-methoxyphenyl)-2H-chromene (23c).

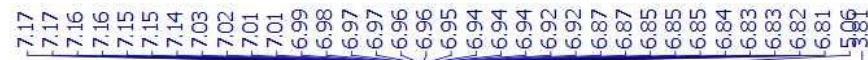<smiles>COc1ccc(C2=C(c3ccc(F)cc3)COc3ccccc32)cc1</smiles> 
(400 $\left.\mathrm{MHz}, \mathrm{CDCl}_{3}\right)$

Figure S97. ${ }^{1} \mathrm{H}$ NMR of 3-(4-Fluorophenyl)-4-(4-methoxyphenyl)-2H-chromene (24c).

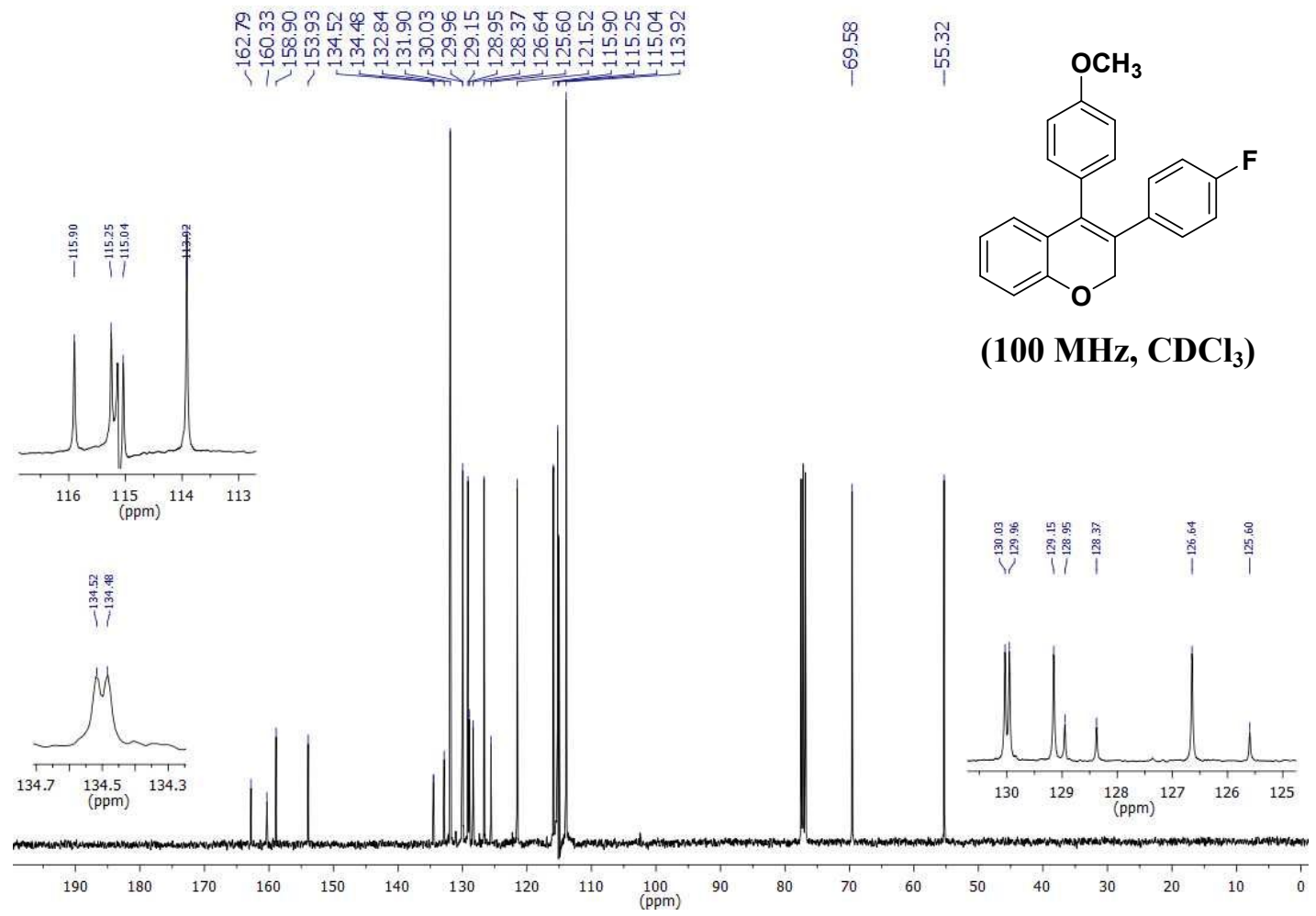

Figure S98. ${ }^{13} \mathrm{C}$ NMR of 3-(4-Fluorophenyl)-4-(4-methoxyphenyl)-2H-chromene (24c).<smiles>COc1ccc(C2=C(c3ccc(F)cc3)COc3ccccc32)cc1</smiles> 


\section{(375 MHz, $\left.\mathrm{CDCl}_{3}\right)$}

Figure S99. ${ }^{19}$ F NMR of 3-(4-Fluorophenyl)-4-(4-methoxyphenyl)-2 $H$-chromene (24c).

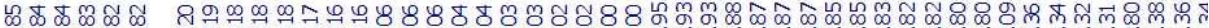

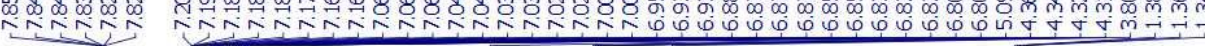<smiles>CCOC(=O)c1ccc(C2=C(c3ccc(OC)cc3)c3ccccc3OC2)cc1</smiles> 


\section{(400 MHz, $\left.\mathrm{CDCl}_{3}\right)$}

Figure S100. ${ }^{1} \mathrm{H}$ NMR of Ethyl 4-(4-(4-Methoxyphenyl)-2H-chromen-3-yl)benzoate (25c).

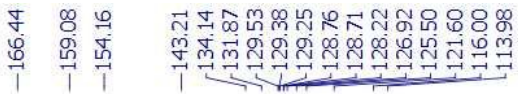

$\begin{array}{lll}\hat{m} & \hat{0} & \vec{m} \\ 0 & \overrightarrow{0} & 0 \\ 0 & 0 & 0\end{array}$

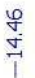<smiles>CCOC(=O)c1ccc(C2=C(c3ccc(OC)cc3)c3ccccc3OC2)cc1</smiles>

$\left(100 \mathrm{MHz}, \mathrm{CDCl}_{3}\right)$

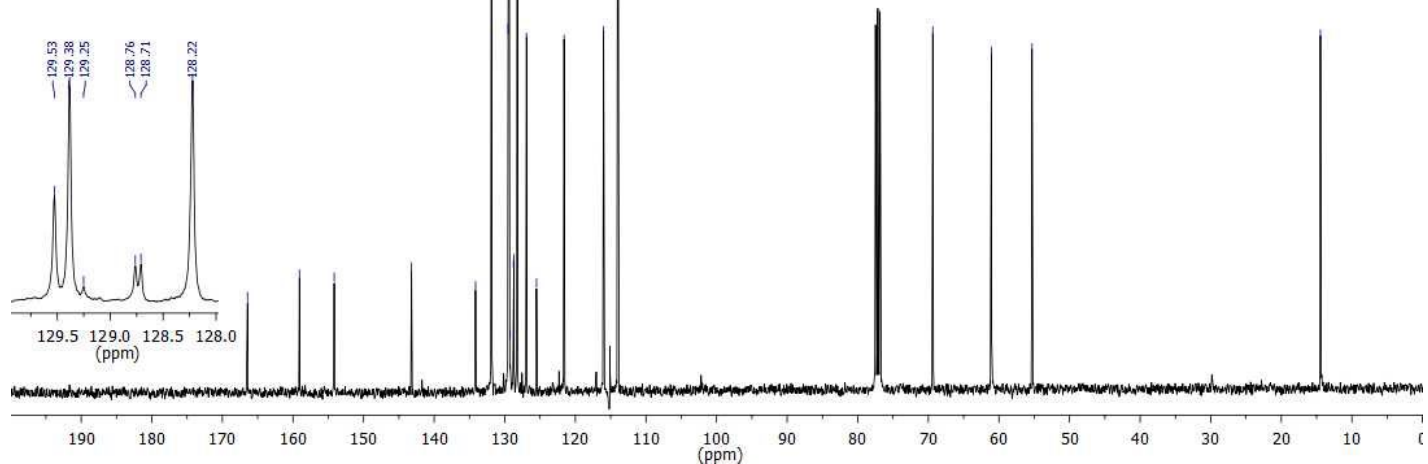

Figure S101. ${ }^{1} \mathrm{H}$ NMR of Ethyl 4-(4-(4-Methoxyphenyl)-2H-chromen-3-yl)benzoate (25c).

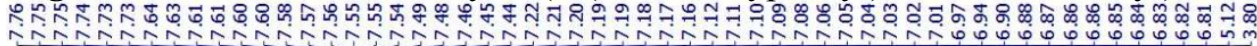<smiles>COc1ccc(C2=C(c3ccc(C(=O)c4ccccc4)cc3)COc3ccccc32)cc1</smiles>

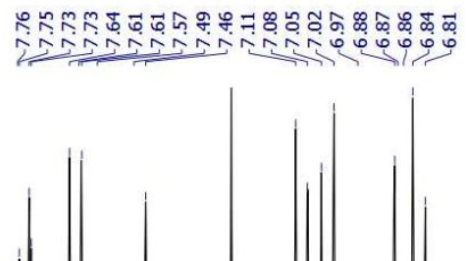


(300 MHz, $\left.\mathrm{CDCl}_{3}\right)$

Figure S102. ${ }^{1} \mathrm{H}$ NMR of (4-(4-(4-methoxyphenyl)-2H-chromen-3-yl)phenyl)(phenyl)methanone (26c).<smiles>COc1ccc(C2=C(c3ccc(C(=O)c4ccccc4)cc3)COc3ccccc32)cc1</smiles>

(75 MHz, $\left.\mathrm{CDCl}_{3}\right)$

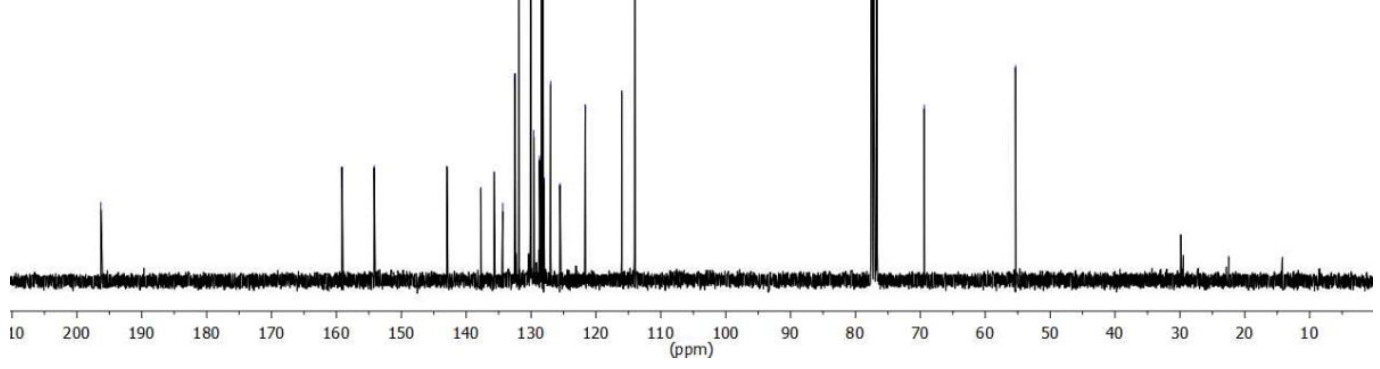

Figure S103. ${ }^{13} \mathrm{C}$ NMR of (4-(4-(4-methoxyphenyl)-2H-chromen-3-yl)phenyl)(phenyl)methanone (26c).

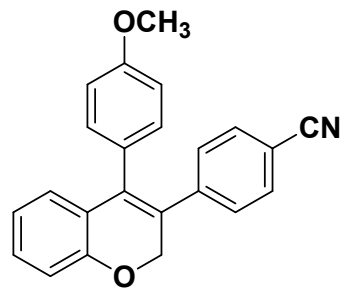


$\left(500 \mathrm{MHz}, \mathrm{CDCl}_{3}\right)$

Figure S104. ${ }^{1} \mathrm{H}$ NMR of 4-(4-(4-methoxyphenyl)-2H-chromen-3-yl)benzonitrile (27c).

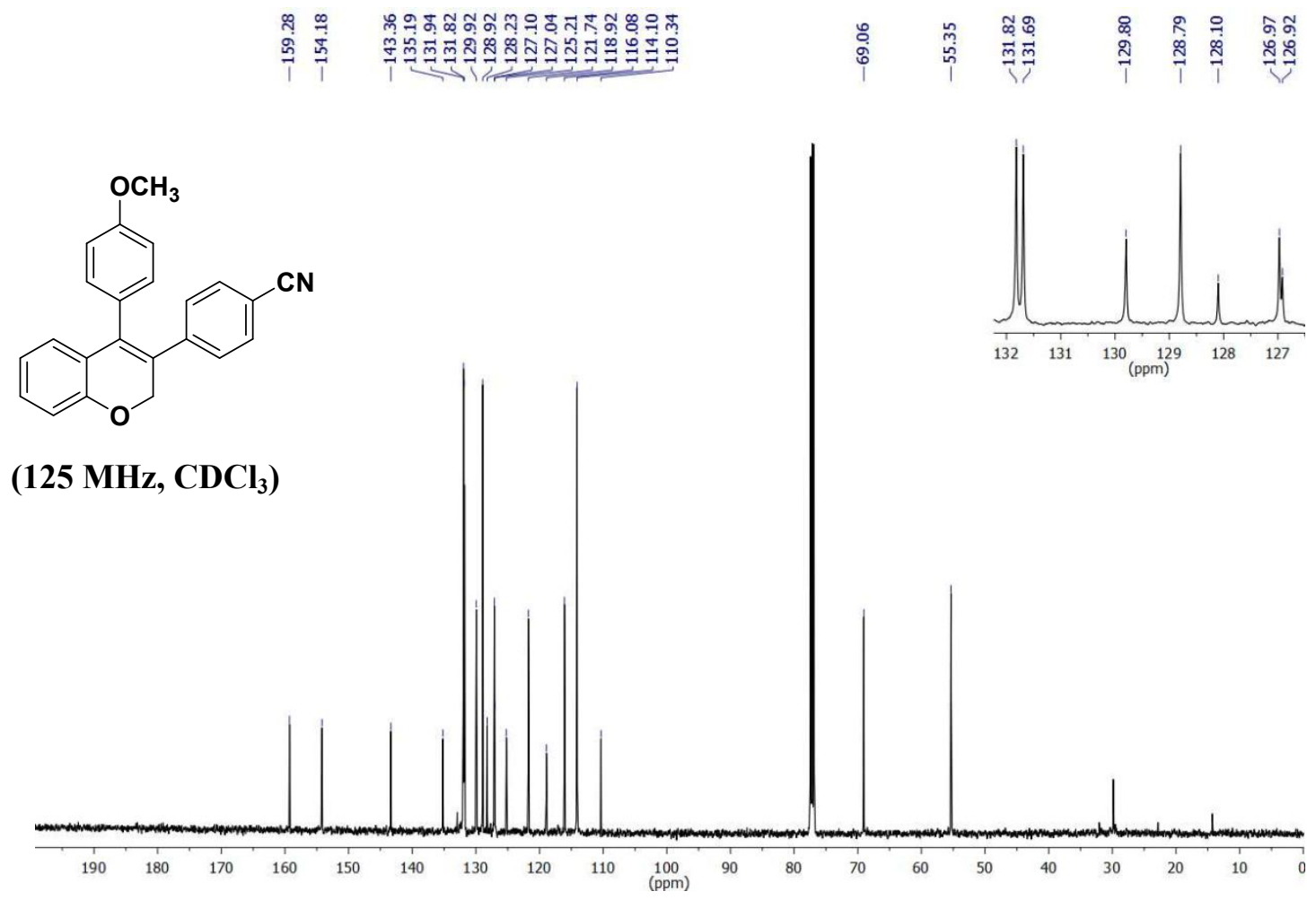

Figure S105. ${ }^{13} \mathrm{C}$ NMR of 4-(4-(4-methoxyphenyl)-2H-chromen-3-yl)benzonitrile (27c).

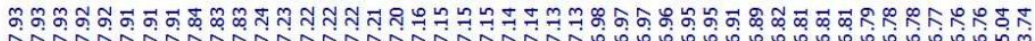

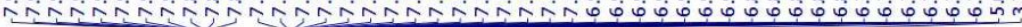


<smiles>COc1ccc(C2=C(c3cccc([N+](=O)[O-])c3)COc3ccccc32)cc1</smiles>

$\left(500 \mathrm{MHz}, \mathrm{CDCl}_{3}\right)$

Figure S106. ${ }^{1} \mathrm{H}$ NMR of 4-(4-methoxyphenyl)-3-(3-nitrophenyl)-2H-chromene (28c).

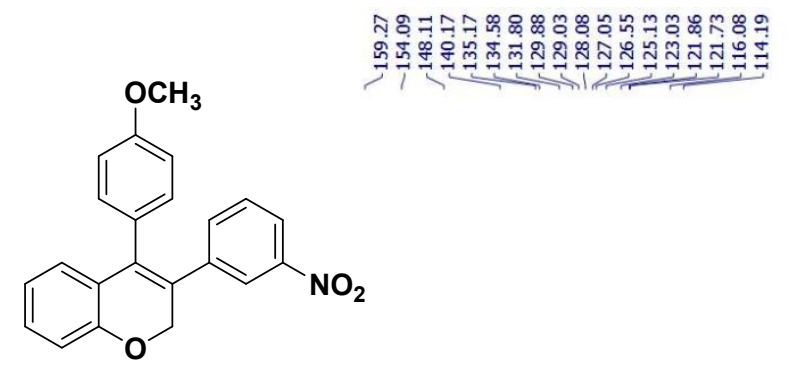

(125 MHz, $\left.\mathrm{CDCl}_{3}\right)$

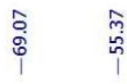
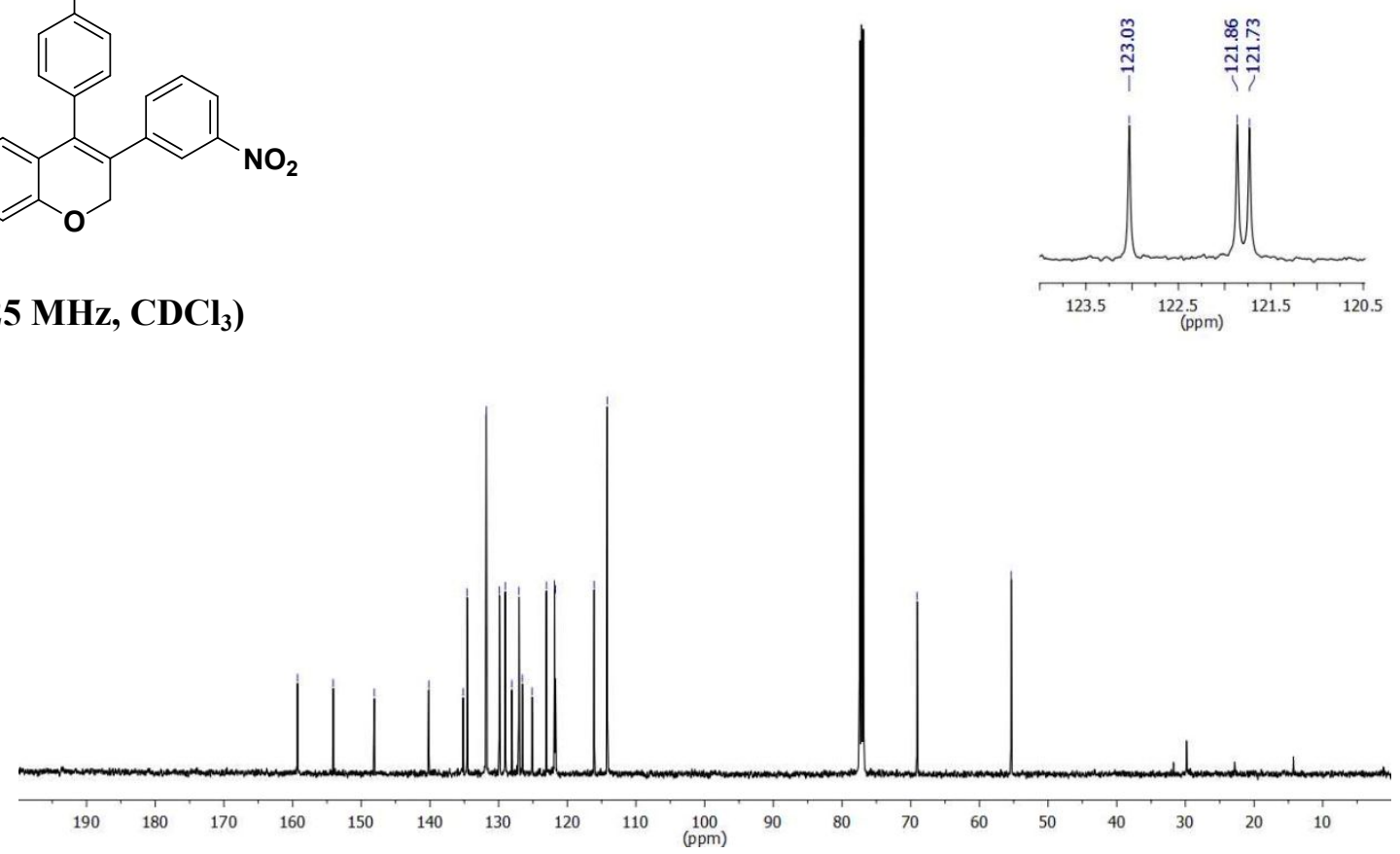

Figure S107. ${ }^{13} \mathrm{C}$ NMR of 4-(4-methoxyphenyl)-3-(3-nitrophenyl)-2 $H$-chromene (28c).
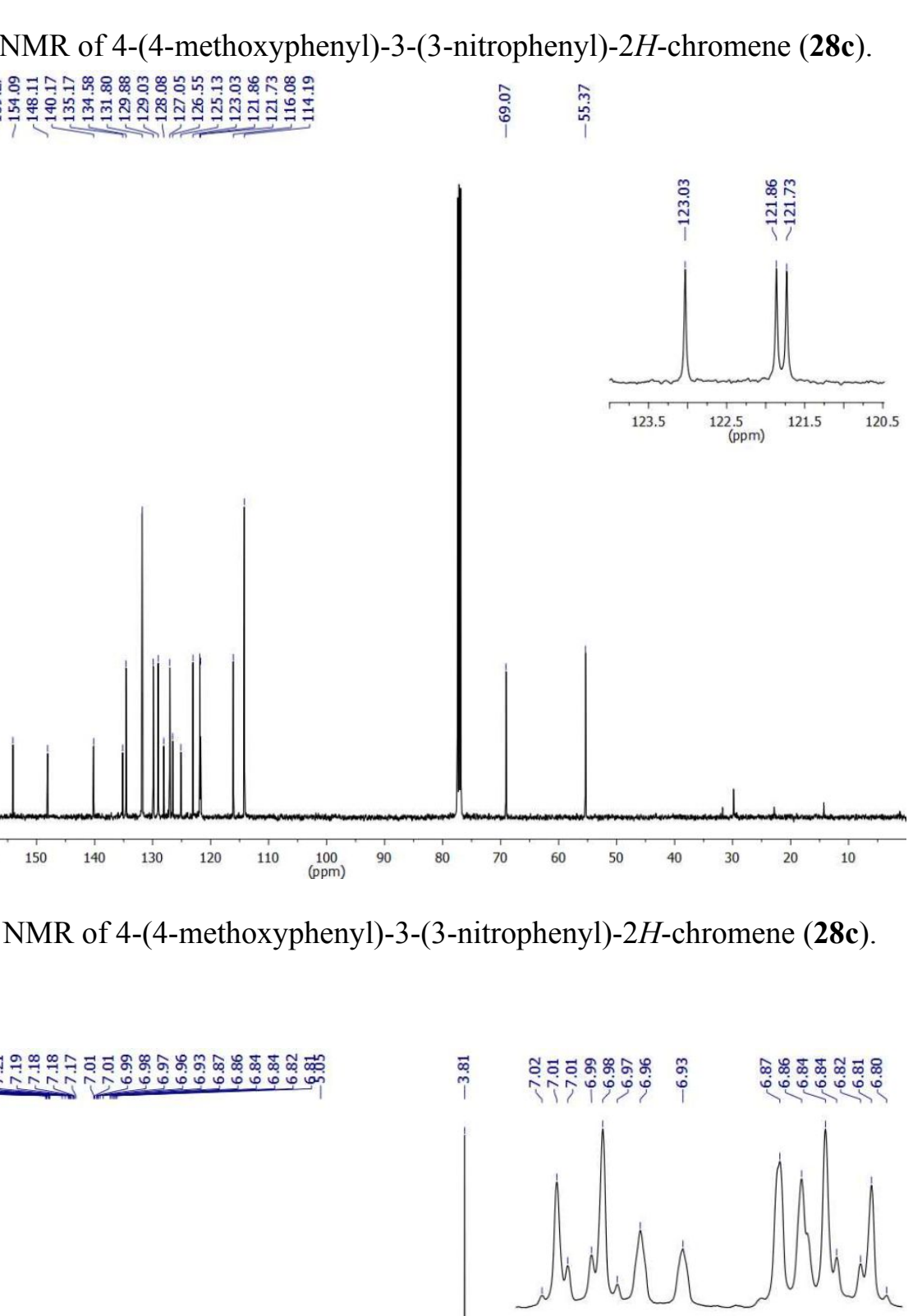
<smiles>COc1ccc(C2=C(c3cccc(C#N)c3)COc3ccccc32)cc1</smiles>

(300 MHz, $\left.\mathrm{CDCl}_{3}\right)$

Figure S108. ${ }^{1} \mathrm{H}$ NMR of 3-(4-(4-methoxyphenyl)-2H-chromen-3-yl)benzonitrile (29c).

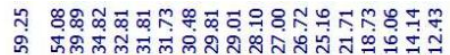

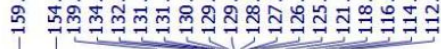

:<smiles>COc1ccc(C2=C(c3cccc(C#N)c3)COc3ccccc32)cc1</smiles>

(75 $\left.\mathrm{MHz}, \mathrm{CDCl}_{3}\right)$

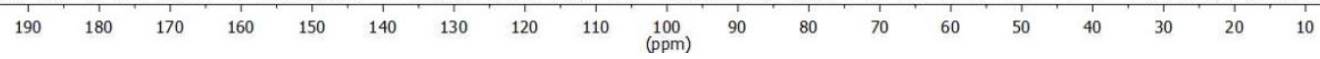

Figure S109. ${ }^{13} \mathrm{C}$ NMR of 3-(4-(4-methoxyphenyl)-2H-chromen-3-yl)benzonitrile (29c).

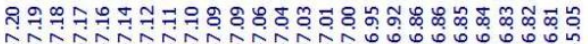

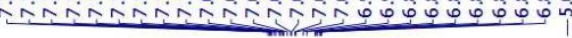

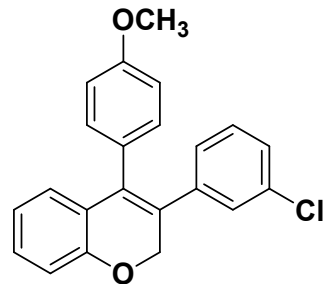


(300 MHz, $\left.\mathrm{CDCl}_{3}\right)$

Figure S110. ${ }^{1} \mathrm{H}$ NMR of 3-(3-chlorophenyl)-4-(4-methoxyphenyl)-2H-chromene (30c).
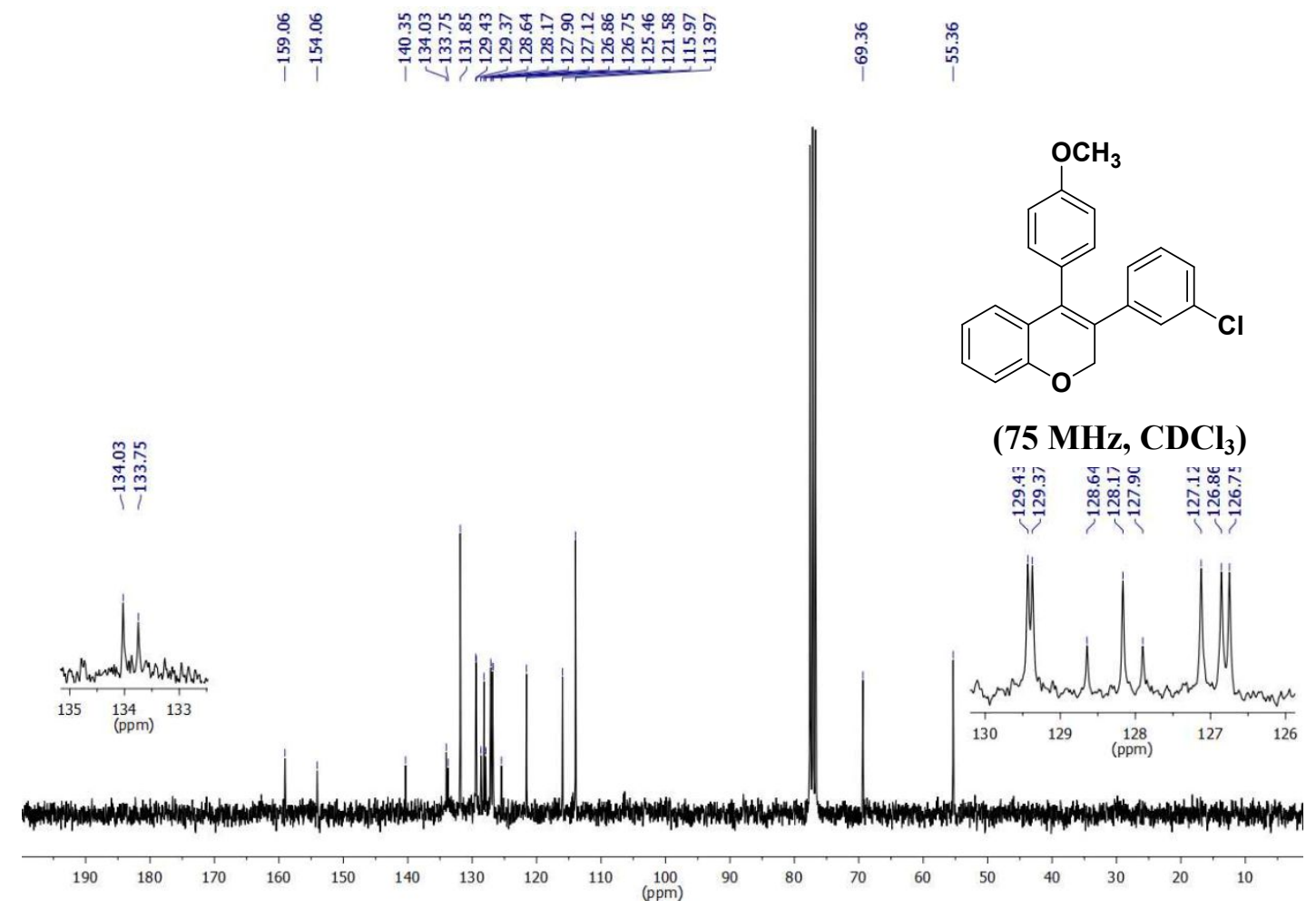

Figure S111. ${ }^{13} \mathrm{C}$ NMR of 3-(3-chlorophenyl)-4-(4-methoxyphenyl)-2 $H$-chromene (30c).

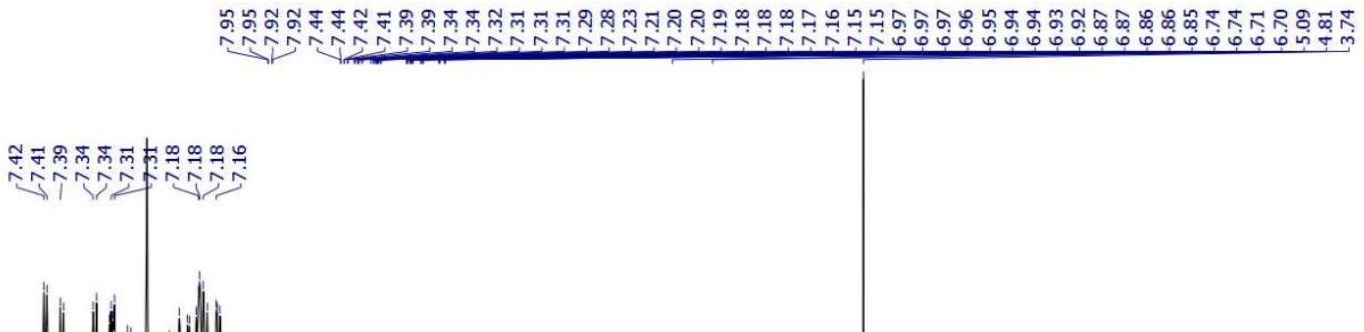




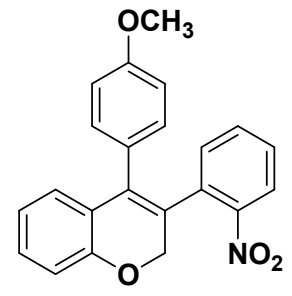

(300 MHz, $\left.\mathrm{CDCl}_{3}\right)$

Figure S112. ${ }^{1} \mathrm{H}$ NMR of 4-(4-methoxyphenyl)-3-(2-nitrophenyl)-2H-chromene (31c).
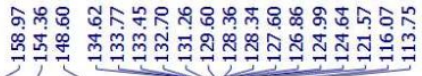

๗ัก

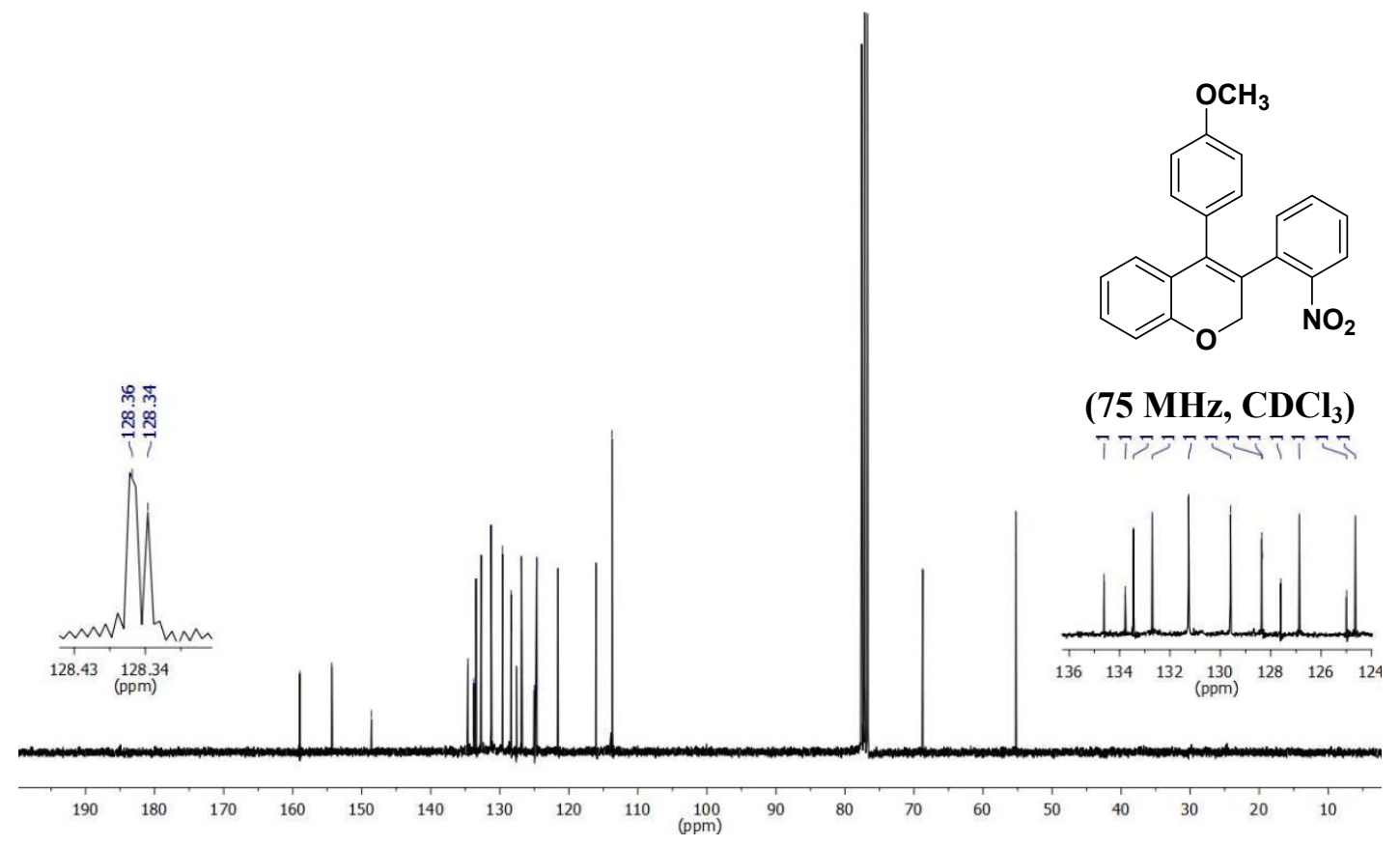

Figure S113. ${ }^{13} \mathrm{C}$ NMR of 4-(4-methoxyphenyl)-3-(2-nitrophenyl)-2H-chromene (31c). 